NIST Special Publication 1170

\title{
Documentation for Reference Material (RM) 8820: A Versatile, Multipurpose Dimensional Metrology Calibration Standard for Scanned Particle Beam, Scanned Probe and Optical Microscopy
}

\author{
Michael T. Postek \\ Andras E. Vladar \\ Bin Ming \\ Benjamin Bunday
}

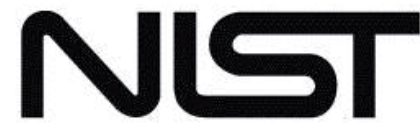

National Institute of Standards and Technology U.S. Department of Commerce 
Page left intentionally blank 
NIST Special Publication 1170 Documentation for Reference Material (RM) 8820: A Versatile, Multipurpose Dimensional Metrology Calibration Standard for Scanned Particle Beam, Scanned Probe and Optical Microscopy

Michael T. Postek

Andras E. Vladar

Bin Ming

Semiconductor and Dimensional Metrology Division

Physical Measurement Laboratory

Benjamin Bunday

International SEMATECH

Albany, New York

http://dx.doi.org/10.6028/NIST.SP.1170

February 2014

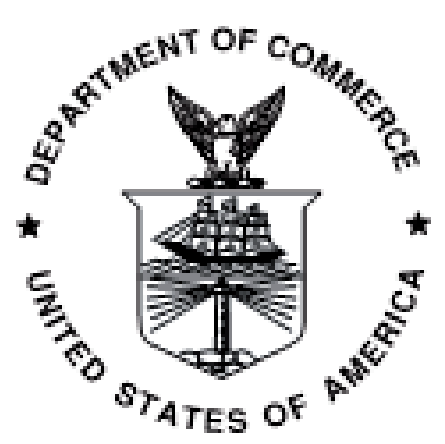

U.S. Department of Commerce

Penny Pritzker, Secretary

National Institute of Standards and Technology Patrick D. Gallagher, Under Secretary of Commerce for Standards and Technology and Director 
Certain commercial entities, equipment, or materials may be identified in this document in order to describe an experimental procedure or concept adequately. Such identification is not intended to imply recommendation or endorsement by the National Institute of Standards and Technology, nor is it intended to imply that the entities, materials, or equipment are necessarily the best available for the purpose.

National Institute of Standards and Technology Special Publication 1170

Natl. Inst. Stand. Technol. Spec. Publ. 1170, 313 pages (February 2014)

CODEN: NSPUE2

http://dx.doi.org/10.6028/NIST.SP.1170 


\section{Table of Contents}

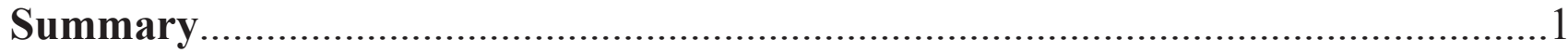

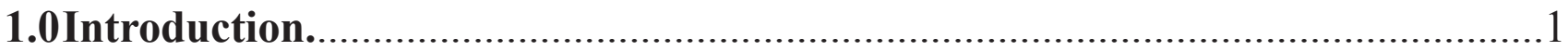

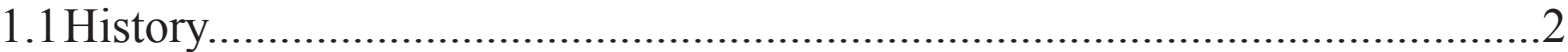

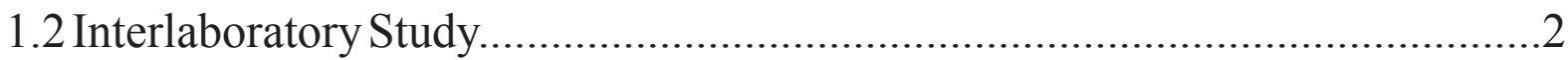

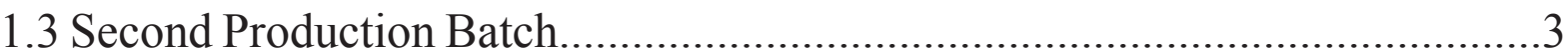

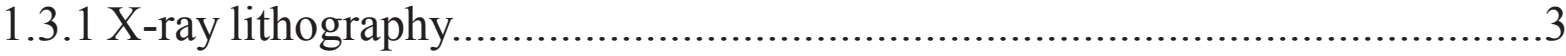

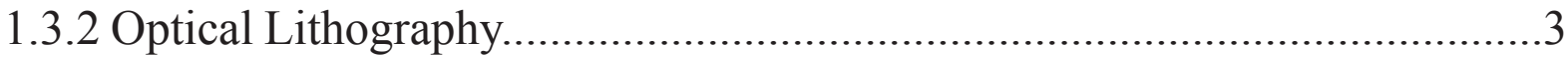

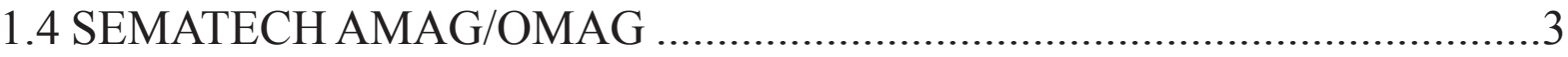

1.5 Reference Material vs. Standard Reference Material......................................

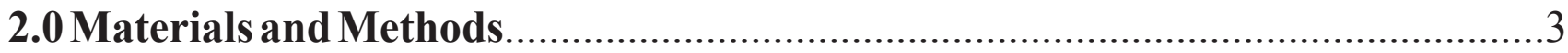

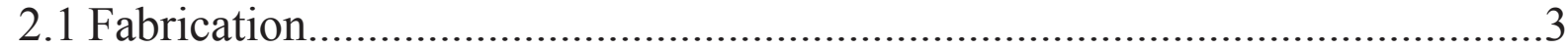

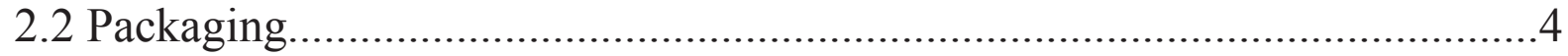

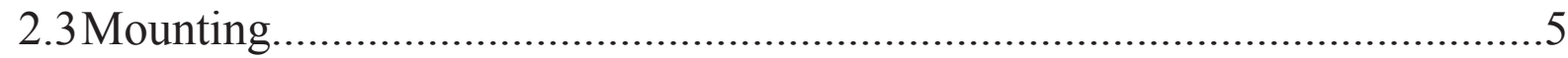

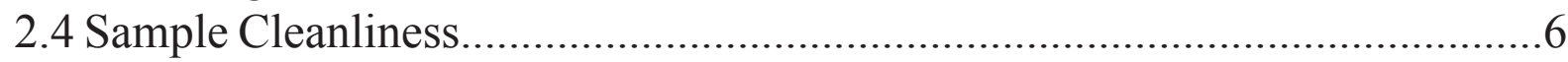

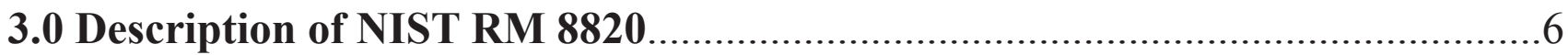

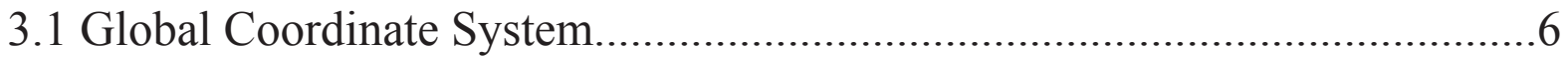

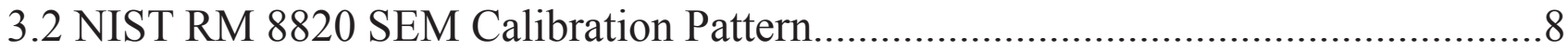

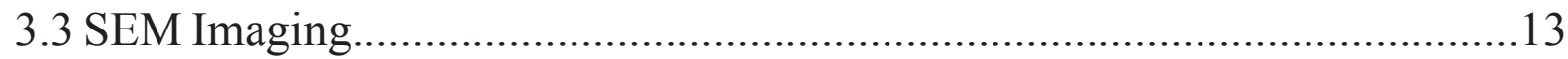

3.4 Reference Scale Calibration Values.........................................................13

3.5 NIST Pitch Calibration Software Program. ...............................................13

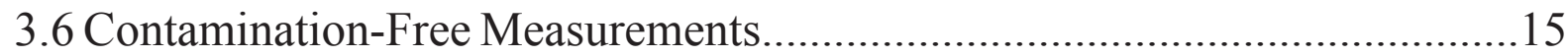

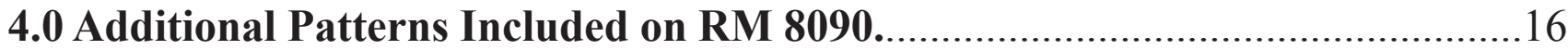

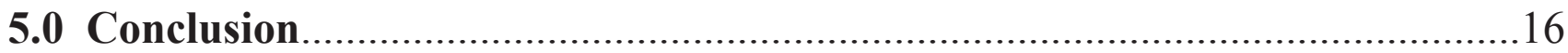

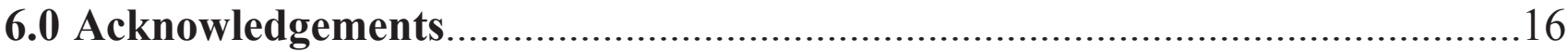

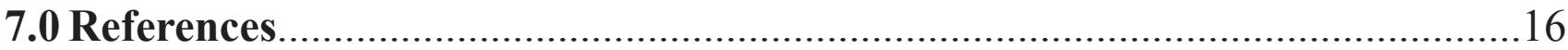




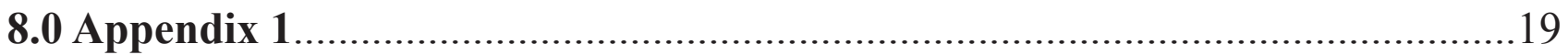

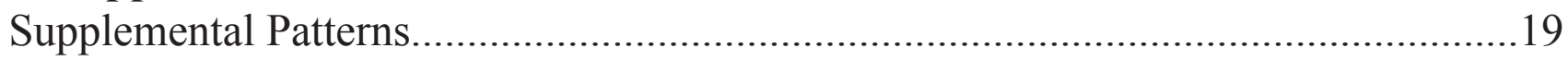

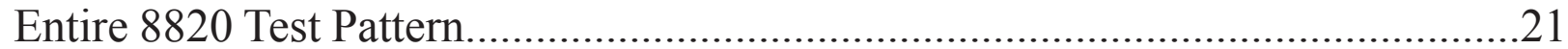

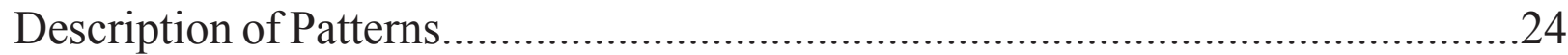

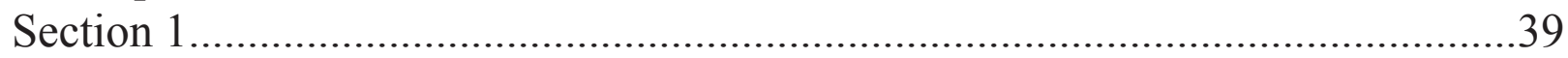

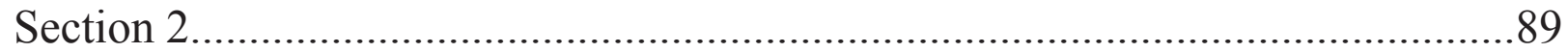

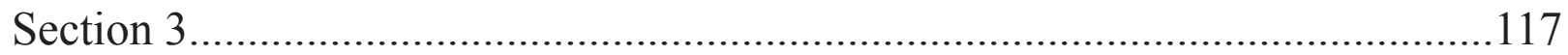

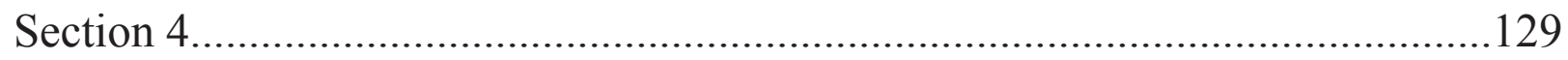

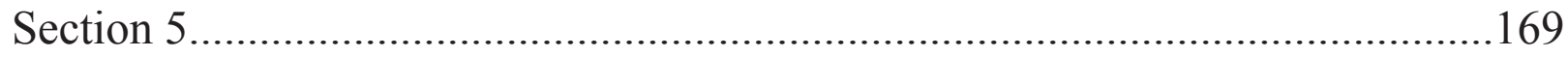

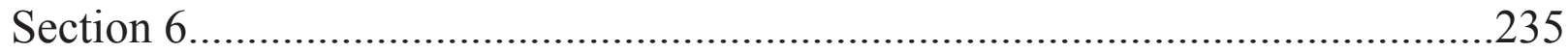

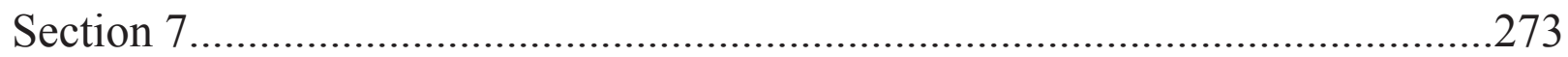




\title{
Documentation for Reference Material (RM) 8820: A Versatile, Multipurpose Dimensional Metrology Calibration Standard for Scanned Particle Beam, Scanned Probe and Optical Microscopy
}

\author{
Michael T. Postek, Andras E. Vladar, Bin Ming 1,2 \\ National Institute of Standards and Technology, \\ Gaithersburg, MD 20899 \\ and \\ Benjamin Bunday \\ International SEMATECH, 257 Fuller Rd, Suite 2200 \\ Albany, NY 12203 USA
}

\begin{abstract}
SUMMARY
Reference Materials (RM) 8820 are multipurpose instrument calibration standard available from NIST. These are dimensional standards initially developed to replace the out of stock RM 8090 used for $X$ and $Y$ scale calibrations of scanned particle beam microscopes. RM 8820 are single, diced $20 \mathrm{~mm}$ x $20 \mathrm{~mm}$ chip from a $200 \mathrm{~mm}$ silicon wafer. There are four NIST RM 8820 patterns on the chip. Surrounding the RM 8820 patterns are many additional test patterns and test structures. These test structures were designed through the NIST collaboration with the SEMATECH Advanced Metrology (AMAG) and Overlay Metrology (OMAG) Advisory Groups. The test structures of RM 8820 were lithographically patterned on a silicon chip. These patterns can also be used for calibration and testing of instruments, other than a scanned particle beam microscopes, such as optical and scanned probe microscopes. In addition to scale calibration, RM 8820 can be used for many other purposes such as stage and scan linearity checking, and instrument contamination testing. This document describes the 8820 Reference Materials, many of the additional test patterns and structures, as well as, some of the additional applications for which this standard can be employed.
\end{abstract}

Keywords: standards, scanning electron microscope, traceability, magnification, field of view, scale, calibration, scatterometry, overlay, linearity

\section{INTRODUCTION}

NIST Reference Material (RM) 8820 was developed primarily to be used for X and Y scale (magnification) calibrations of scanned particle beam microscopes such as scanning electron (SEM), focused ion beam (FIB) and helium ion (HIM) microscopes from less than 10 times magnification to more than 300000 times magnification, i.e., a horizontal field width $(\mathrm{HFW})^{3}$ from about $10 \mathrm{~mm}$ to smaller than $300 \mathrm{~nm}$ range instrument HFW [1]. The HFW is a more appropriate way to describe what is commonly referred to as instrument magnification, but both terms will be used in this document.

\footnotetext{
${ }^{1}$ Certain commercial equipment is identified in this work to adequately describe the experimental procedure. Such identification does not imply recommendation or endorsement by the National Institute of Standards and Technology, nor does it imply that the equipment identified is necessarily the best available for the purpose.

${ }^{2}$ Contribution of the National Institute of Standards and Technology; not subject to copyright.

${ }^{3}$ Horizontal Field Width (HFW). The generally used term magnification has become meaningless terminology. This is because of the myriad of ways an image can be manipulated after these data have been taken and stored. Therefore, the term horizontal field width or HFW is a more appropriate term and has been adopted in this work to reflect this more correct terminology.
} 
There are four NIST RM 8820 patterns located on the reference material. These four patterns are described in the first part of this document. Surrounding each of the RM 8820 patterns can be found many additional test patterns and structures. These patterns are described in the SUPPLEMENTAL PATTERNS section of this document. These test structures were designed through the NIST collaboration with SEMATECH and its Advanced Metrology (AMAG) and the Overlay Metrology (OMAG) Advisory Groups. All of the test structures of RM 8820 were lithographically patterned on a silicon chip and can also be used for calibration and testing of other type of instruments such as: scatterometers and optical and scanned probe microscopes. Using RM 8820, all microscopes within a laboratory can be calibrated to the same reference sample. In addition to scale calibration, RM 8820 can be used for stage and scan linearity checking and instrument contamination testing (as described in a later section of this document).

The overall RM 8820 was fabricated at SEMATECH, Austin, TX. This overall sample is the culmination of many years of effort to provide the industry with low-cost calibration standards for laboratory and manufacturing metrology instrumentation. This document describes the 8820 standard, many of the additional test patterns and structures, and outlines a procedure for using the RM for contamination testing.

\subsection{History}

The first lithographically produced NIST Reference Material (RM) for SEM magnification calibration was introduced to the microscopy community in 1988 [2] and was released as a NIST Reference Material in 1995 - RM 8090 [3]. The RM 8090 samples were fabricated using direct-write electron beam lithography and a metal lift-off process on a silicon wafer substrate. The pattern consisted of a layer of $10 \mathrm{~nm}$ titanium and $40 \mathrm{~nm}$ palladium on a $10 \times 10 \mathrm{~mm}$ silicon chip. The edge roughness of the $200 \mathrm{~nm}$ pitch structures was measured to be $5 \mathrm{~nm}$ or less. The lift off technique resulted in an edge roughness of approximately $4 \mathrm{~nm}$.

The initial NIST RM 8090 sample was a product of several years of research effort. The three major design constraints were 1) adequate fidelity for the smallest line structures (200 $\mathrm{nm}$ pitch); 2) the ability to view the sample at both high and low accelerating voltages [4], and 3) materials that are fully compatible with the then current complementary metal-oxide-semiconductor (CMOS) production. Therefore, gold was a material that could not be used. These fundamental requirements were achieved on proof-of-concept samples that were initially fabricated by direct write e-beam lithography and manually processed at the Cornell National Nanofabrication Facility (NNF) in collaboration with Dr. Richard Tiberio [2,5]. Following the initial proof of concept, a second batch of evaluation (20) samples was fabricated by Dr. Tiberio and NIST circulated them to the community for technical comments and review.

The generation of a useful reference material for the United States requires the ability for NIST to stock and make available a number of samples sufficient to meet the anticipated demands of the customer base. With literally hundreds of scanning electron microscopes in use at that time, faster and more efficient ways of fabrication needed to be sought. Standards such as RM 8090 or RM 8820 should be made in bulk quantities and must be inexpensive enough to be used on the "shop floor." Through the efforts of Ms Marilyn Hoy Bennett and Dr. Brian Newell, of Texas Instruments (TI), Dallas, they were able to convince TI to assist NIST in fabricating the first ever production batch of the reference materials. Using direct write electron beam technology and full wafer lithographic processing capabilities, approximately 150 samples were initially fabricated $[6,7,8]$. The major portion of these samples was placed into stock within the Office of Standard Reference Materials (OSRM) and also a number of the samples were distributed to about 60 laboratories across the United States in an interlaboratory study [9].

1.2 Interlaboratory Study. Several of the initial RM 8090 samples were distributed to laboratories in industry, academia and government as an interlaboratory study with the goal of assessing the impact of the RM on the customer base. Approximately 60 laboratories across the United States agreed to participate in the study [9]. The major results of this study clearly outlined the current calibration deficiencies and need for accurate instrument calibration [9]. Use of the new standard demonstrated that scanning electron microscopes, currently in use, were generally mis-calibrated as much as $60 \%$. Even instruments within the same laboratory were as much as $15 \%$ mis-calibrated to each other. This study opened the eyes of the SEM researchers regarding the need to calibrate properly the instrumentation both in the laboratory and for manufacturing. 
1.3 Second Production Batch. The first production run of RM 8090 sold out rapidly and it became necessary to look again into the technology pool for a solution. TI agreed to provide another batch of 50 samples as an interim solution. Therefore, a second release of RM 8090 samples occurred. But during the interim between RM fabrication batches, several internal changes at Texas Instruments occurred, resulting in the inability for TI to fabricate any additional batches of the materials. Subsequently, other companies having direct-write e-beam capabilities volunteered to assist NIST but were unable to meet the fidelity requirements in the materials needed for the sample. Other avenues for fabrication needed to be developed.

1.3.1 X-ray Lithography. During the technology search, x-ray lithography was considered to be a viable alternative technology. X-ray lithography was potentially a very high-fidelity and rapid throughput technology lending itself to mass fabrication of the RMs. Utilizing x-ray lithography in the fabrication of the RMs would be extremely cost-effective and many samples could be made available at a low cost. The complication and eventual weakness in this technique was the fabrication of the x-ray mask. The fabrication of the x-ray lithographic mask was unsuccessful and it became necessary to look elsewhere for a solution.

1.3.2 Optical Lithography. Several other attempts were made to fabricate the RM sample through electron beam lithography by a number of other laboratories. These attempts proved to be unsuccessful and not cost-effective. Fortunately, optical lithography continued along the path of Moore's law as a viable technology. Through the strong collaboration between NIST and SEMATECH, state-of-the art phase-shifting masks were fabricated and experiments were done to optimize the lithography needed for the fidelity of the fine lines of the RM. This was accomplished and 100 samples were delivered to the NIST Office of Standard Reference Materials (OSRM). Since the overall pattern on the chip was markedly different than the previous RM 8090 samples, the new RM was named RM 8820.

1.4 SEMATECH AMAG/OMAG. RM 8820 was a product of the strong SEMATECH Advanced Metrology Advisory Group (AMAG)/NIST collaboration. The overall chip was designed with the intent of not only solving the NIST need for a new reference material but also the semiconductor industry's need for additional calibration and test patterns. Therefore, the four NIST patterns found on the chip are surrounded by numerous other patterns specifically designed for applications beyond just SEM calibration (Figure 1). Therefore, this document not only describes the RM 8820 patterns, but also the majority of the supplemental patterns in order to offer this standard to a wider community of users (see: Suppelmental Patterns Section).

1.5 Reference Material vs. Standard Reference Material. In order to get the new standard to the industrial users as rapidly as possible, the first introduction of this standard was as an RM (an SRM version is currently being developed and will be discussed later). A NIST Standard Reference Material (SRM) is at the pinnacle of the reference material chain. An SRM has been highly researched and all sources of uncertainty have been explored leading to as low a measurement uncertainty as possible. NIST Reference Materials (RM) have been highly researched, but all of the sources of measurement uncertainty have not been fully explored or accounted for. Therefore, the measurement uncertainty is somewhat higher.

\subsection{Materials and Methods}

2.1 Fabrication. The RM 8820 samples were fabricated on $200 \mathrm{~mm}$ silicon ( $\mathrm{Si}$ ) wafers using $193 \mathrm{~nm}$ extreme ultraviolet light lithography and a dry-etch process that formed all the patterns from an amorphous Si layer deposited on the silicon substrate with a thin silicon oxide layer between the layers. This $2 \mathrm{~nm}$ thin $\mathrm{SiO}_{2}$ was used as an etch stop. Before the patterns were made, measurements were carried out to determine the thickness of the amorphous Si layer. The average thickness of the amorphous Si layer was found to be $97.3 \mathrm{~nm}$ with a standard deviation $(\sigma)$ of $1.6 \mathrm{~nm}$.

All amorphous silicon patterns exhibit a natural edge unevenness or edge roughness. This is a consequence of the lithography and etch processes. For RM 8820, the processing resulted in an average edge roughness of $6 \mathrm{~nm}$. This edge roughness, however, does not have a very large effect on the pitch determination for calibration if a large enough segment of the lines is included in each pitch measurement. This can be achieved by measuring the center two-thirds of the images taken at suitable magnifications. To assist the user, a pitch calculation software program is available from NIST (upon request) that will calculate the pitch on hundreds or thousands of locations and give a statistical measure of the pitch values (see the description of this program later in this document). 


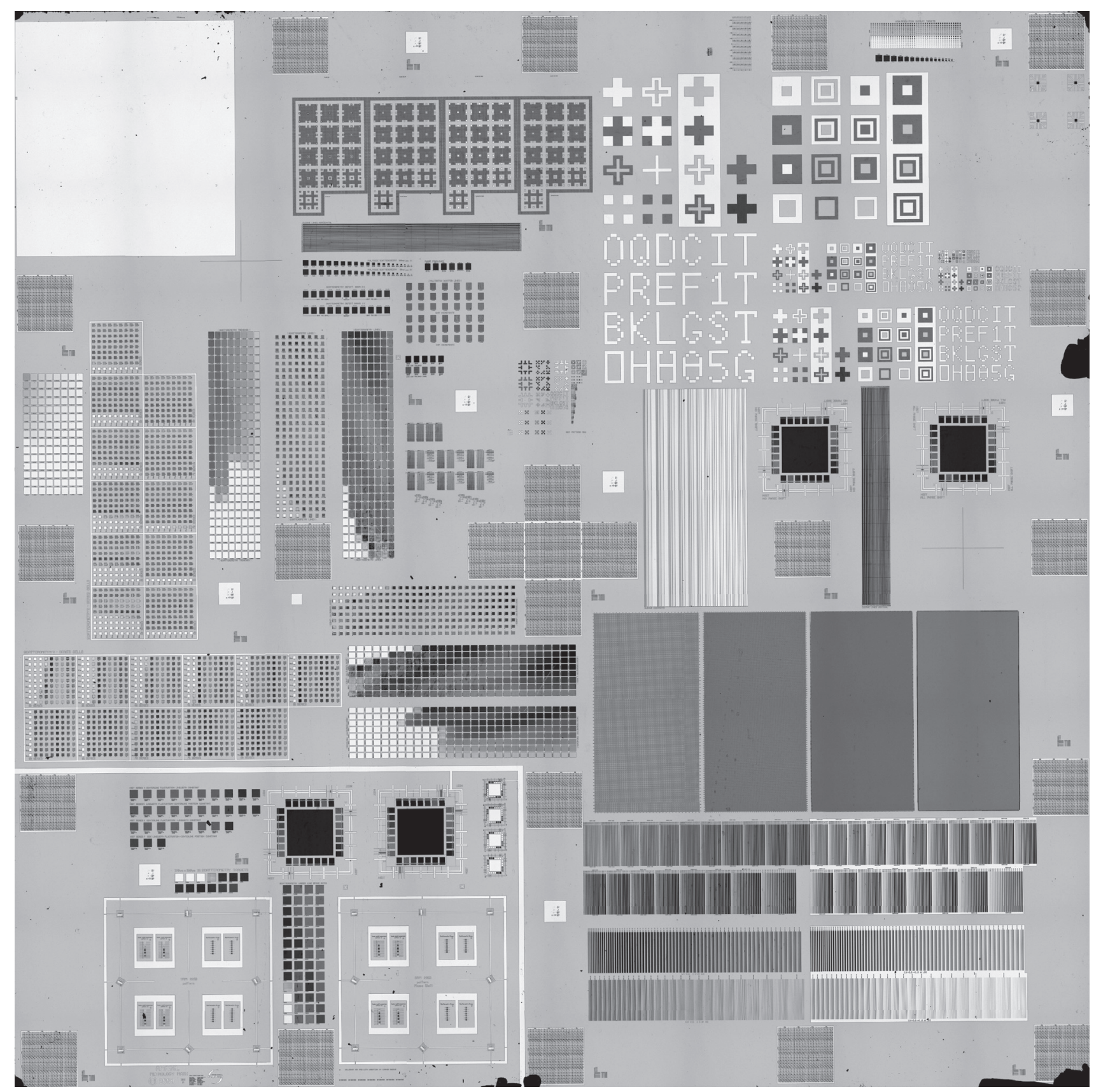

Figure 1. Overall bright field optical micrograph of the RM 8820 sample. (Courtesy of Zeiss, Inc.)

RM 8820 was produced through collaboration with SEMATECH in Austin, TX. An Applied Materials [1] critical dimension (CD) measurement SEM using a thermally assisted field emission source was used at International SEMATECH for automatic measurements of the samples prior to dicing and packaging. Measurements of multiple scans were averaged for the NIST metrology instrument result. The NIST inspection instrument used an average of 64 lines.

2.2 Packaging. Each sample is provided in a box lined with semi-sticky plastic to protect it from damage. It is important to use, preferably conductive plastic tools (readily available from several suppliers) to carefully remove the chip from its 


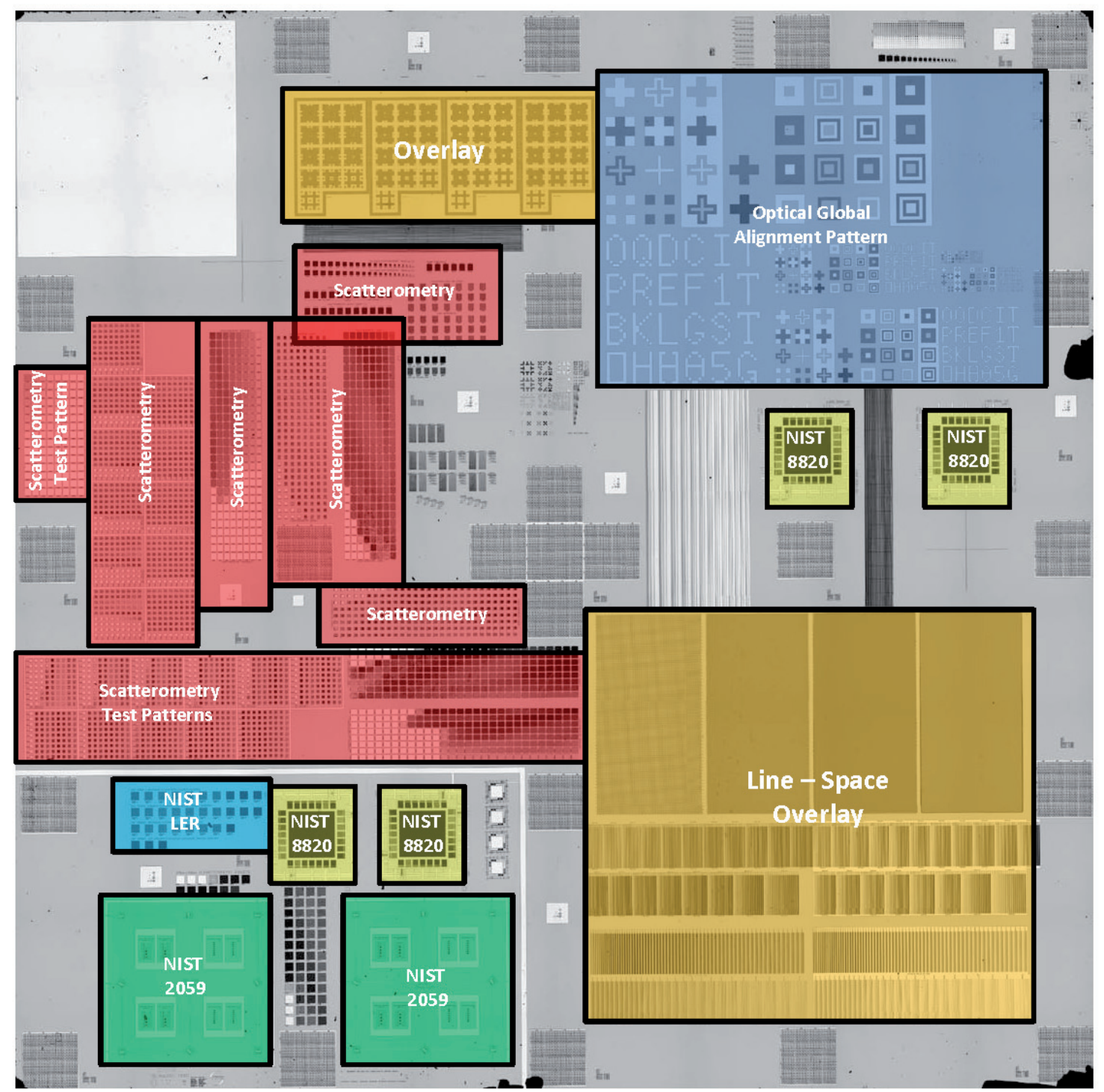

Figure 2. Identification and highlighting of some of the types of calibration and testing patterns available on RM 8820 overlain on the bright field optical micrograph of Figure 1.

box. The semi-sticky plastic will let the user move the chip slowly away from the surface by gently pushing a tool under the bottom of the chip. It takes a few seconds for the semi-sticky plastic to recede and the chip then can be lifted out of the box.

2.3 Mounting. The RM chip is intended to be mounted by the users on the proper stub, wafer or sample holder suitable for their particular instrument. Utmost care should be taken in the handling and mounting of the sample. Spring-loaded fasteners or a very small amount of carbon conductive paste applied at two corners of the chip were found to work well.

Electron beam-induced contamination can be deposited on the sample depending on the handling, instrument cleanliness, electron beam current and accelerating voltage used. Only the user has control over these parameters. It is possible to achieve cleanliness so that after 10 minutes of continuous imaging no visible change in the quality of the image is observed or the measured value of the pitch. This may require the use of instrumentation and methods that are designed to clean the sample and the sample chamber and its vacuum system $[10,11]$. These methods are discussed further, in Sections 2.4 and 3.6 of this document. 


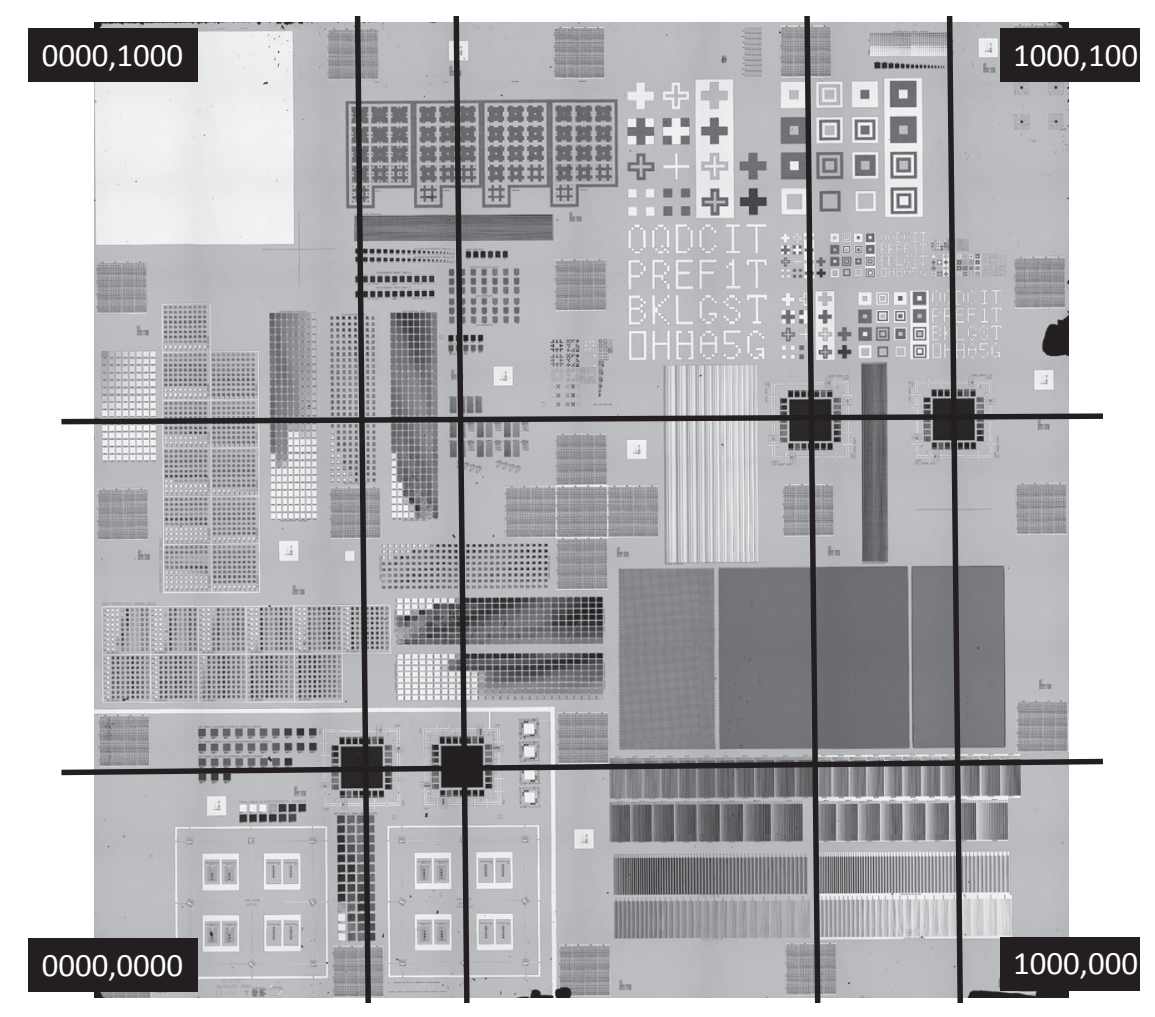

Figure 3. Optical micrograph of the overall RM 8820 with the global grid applied and the four NIST RM 8820 patterns shown at the coordinate locations described in the text.

2.4 Sample Cleanliness. Sample cleanliness and elimination of electron or ion beam-induced contamination are both essential; this is especially true at small HFW (high magnifications). Unless the sample and the microscope are clean enough, (i.e., allowing for ten minutes of continuous imaging or measurement without noticeable sign of contamination), it is likely that erroneous and misleading results will be obtained. The RM artifacts were cleaned after production, but it makes sense to check, and if necessary, clean the sample before it is placed under the particle beam. Later in this document there is a more detailed description of a cleaning procedure that was found to be very useful to clean (even electron beam) contaminated RM 8820 samples and the vacuum space of the microscope.

\subsection{Description of NIST RM 8820}

The overall RM 8820 chip is composed of the NIST patterns and a large number of additional patterns developed through the SEMATECH AMAG and OMAG/NIST collaboration as shown in Figure 1. The overall chip was designed with the intent of solving the NIST and industrial need for a new dimensional reference material for scanned particle beam instrumentation used specifically in semiconductor manufacturing. This reference material is also useful in the calibration of those instruments used for other applications, as well. But the main constraints on the sample and material used for fabrication were dictated by semiconductor processing limitations. In addition, the additional calibration and test patterns identified by the AMAG/ NIST are included and are designed for applications beyond just particle beam instrument calibration. These include atomic force microscope calibration, optical microscope calibration, overlay metrology, line edge roughness measurement, global alignment test patterns, scatterometry test patterns, and a number of additional patterns with the locations shown in Figure 2.

3.1 Global coordinate system. The RM 8820 patterns are found within an overall square pattern on the silicon chip. Most modern SEMs and other instruments have a precise vernier system for the sample drive mechanisms whether they are manual or motorized. Many of the critical dimension (CD) SEMs have automated stages and pattern recognition software. Therefore, a grid coordinate system for precise location of the patterns can be readily developed. 


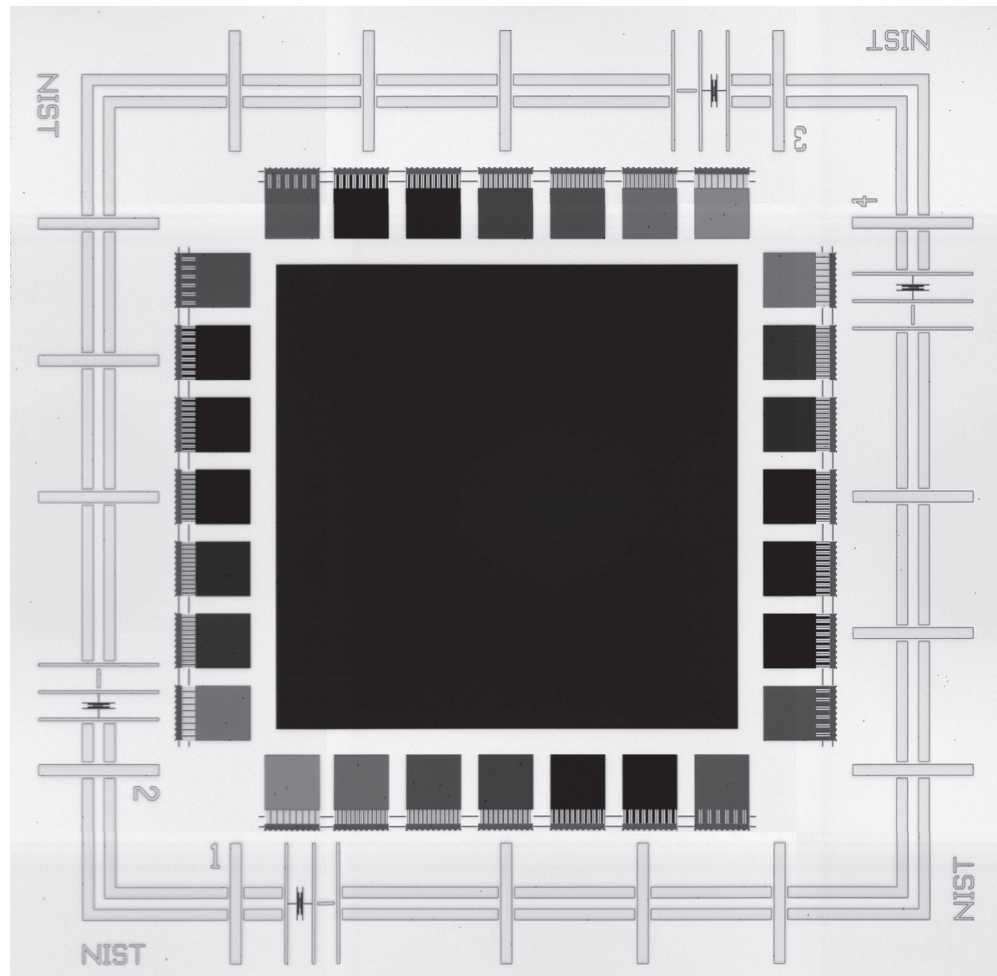

Figure 4. Optical microscope view of the $1.5 \mathrm{~mm}$ by $1.5 \mathrm{~mm}$ RM 8820 test structure.

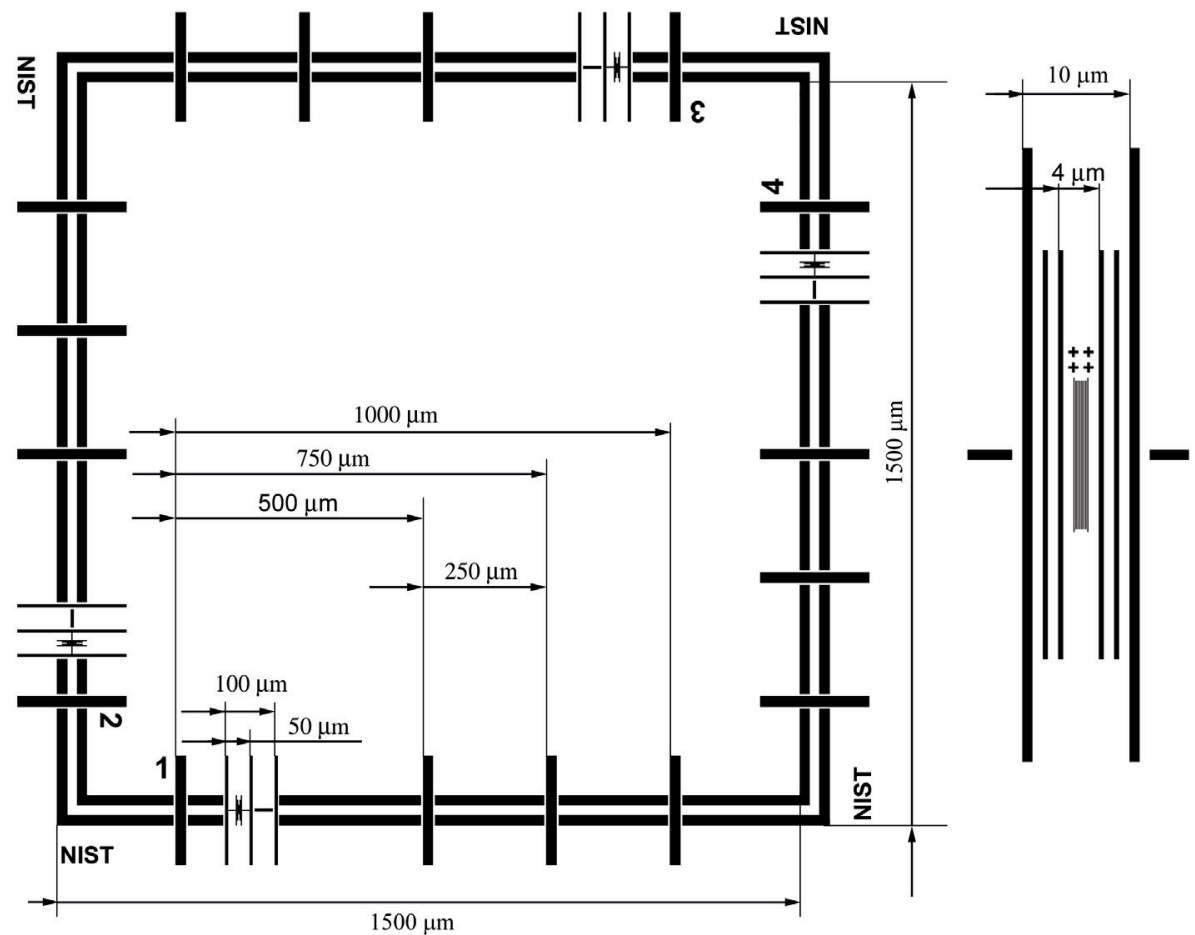

Figure 5. The nominal $X$ and $Y$ direction pitch values for the large structures of RM 8820 . 


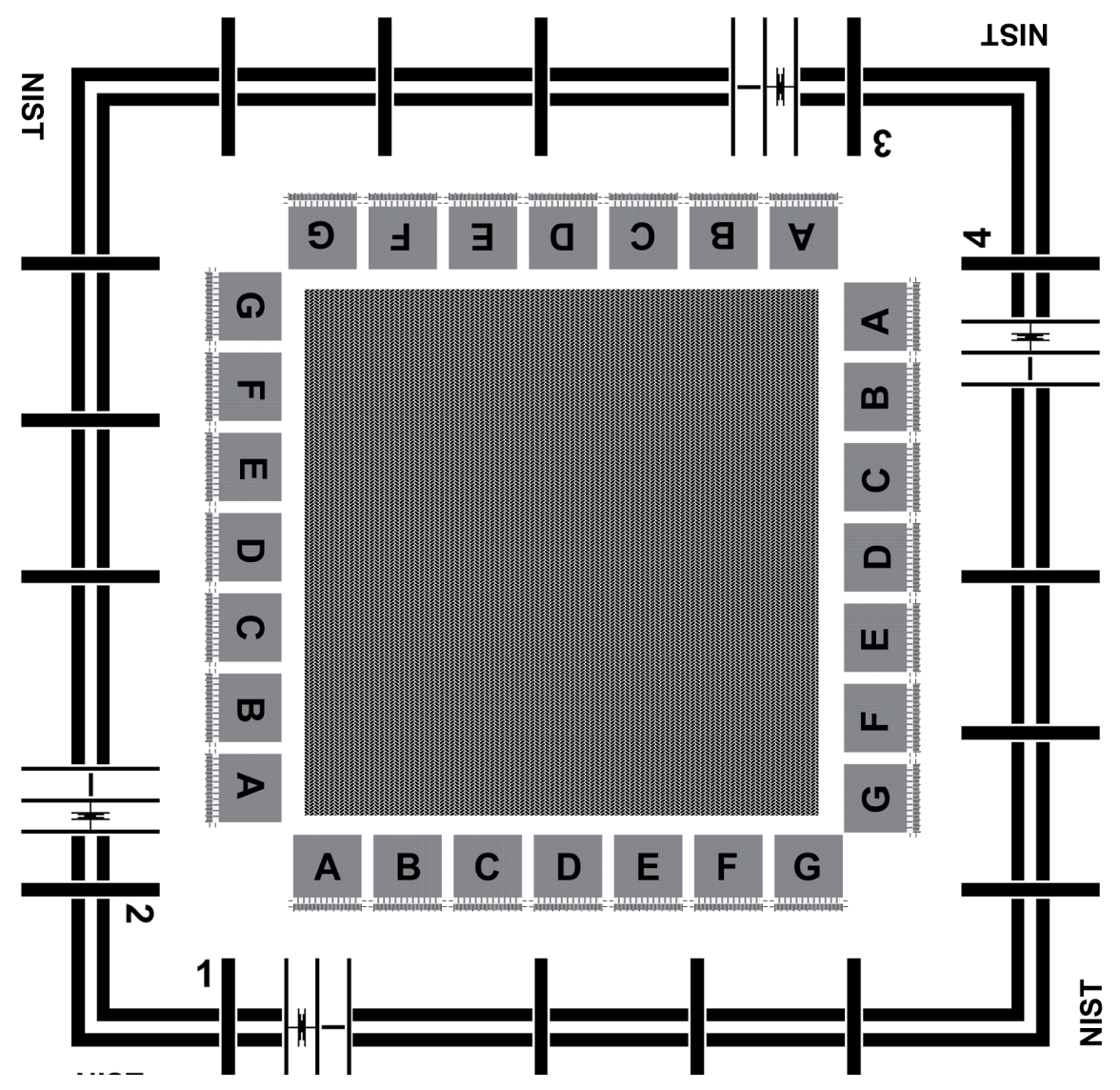

\author{
A $200 \mathrm{~nm}$ pitch \\ B $280 \mathrm{~nm}$ pitch \\ C $400 \mathrm{~nm}$ pitch \\ D $500 \mathrm{~nm}$ pitch \\ E $700 \mathrm{~nm}$ pitch \\ $\mathrm{F} 1 \mu \mathrm{m}$ pitch \\ $\mathrm{G} 2 \mu \mathrm{m}$ pitch
}

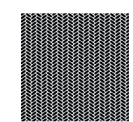

The center area is filled with $250 \mathrm{~nm}$ crosses

on non-connected structures and with a $250 \mathrm{~nm}$ grid on connected structures.

Figure 6. The nominal $X$ and $Y$ direction pitch values for the small A to G structures of RM 8820 .

Figure 3 shows one approach to the development of a coordinate system for locating the patterns. Here, the upper left corner is coordinate $(0000,1000)$; the lower left corner is coordinate $(0000,0000)$; the upper right corner is coordinate $(1000,1000)$ and the lower right is coordinate $(1000,0000)$. The $400 \mathrm{~mm}^{2}$ chip $(20 \mathrm{~mm} \times 20 \mathrm{~mm})$ is then broken into 10000 sub-squares; a calibration structure of interest can be precisely identified according to that grid.

3.2 NIST RM 8820 SEM Calibration Pattern. The NIST particle beam calibration patterns of RM 8820 have been fabricated on the $20 \mathrm{~mm}$ x $20 \mathrm{~mm}$ silicon chip as a portion of a very large set of structures as shown in Figures $1-3$. Note the locations of the four NIST scanning electron microscope calibration patterns (Figure 2). These patterns are $1.5 \mathrm{~mm}$ by $1.5 \mathrm{~mm}$ in size and are marked with letters "NIST." These are readily visible with the naked eye as small bright squares within the large chip. Like its predecessor, RM 8820 patterns have pitches ranging from $200 \mathrm{~nm}$ to $1.5 \mathrm{~mm}$ in both $\mathrm{X}$ and $\mathrm{Y}$ directions (Figure 4).

The four NIST patterns are composed of two versions of the RM 8820 pattern. In one version, the structures are separate from each other; in the other version, all patterns are electrically connected to each other. This makes it possible to connect these patterns to ground potential, if that is deemed by the user to be useful. The measurements done for quality assessment were done on the non-connected patterns. There are two pairs of the connected and non-connected patterns, which all can be used for scale calibration purposes. Within each RM 8820 pattern, there are 4 sets of calibration patterns (2 in the $\mathrm{X}$ direction and 2 in the $\mathrm{Y}$ direction). In the center of all of the RM 8820 patterns, there is a large area of structures for focusing and astigmatism correction and for the measurement of scan linearity.

There are numerous other pitch structures on the RM that can be made into secondary calibration structures once the microscope or other instrument has been initially calibrated. Development of secondary calibration structures is especially 


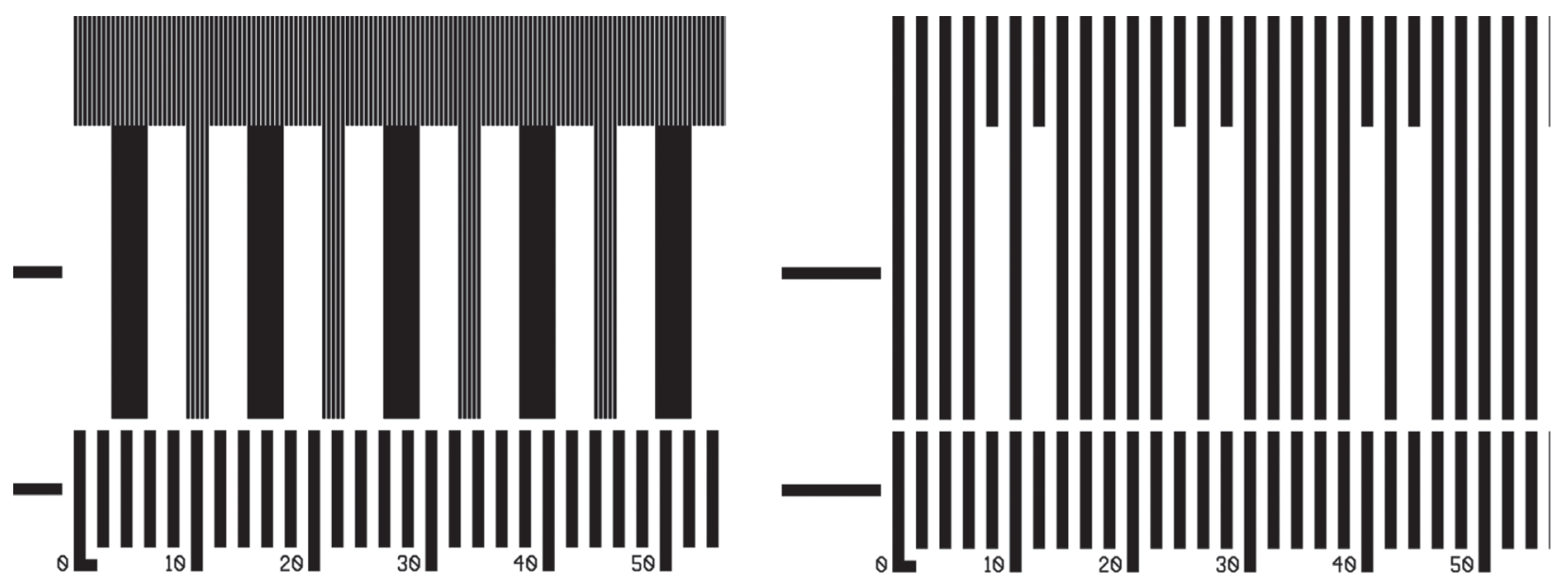

Figure 7. Schematic design of structures A (left) and G (right).

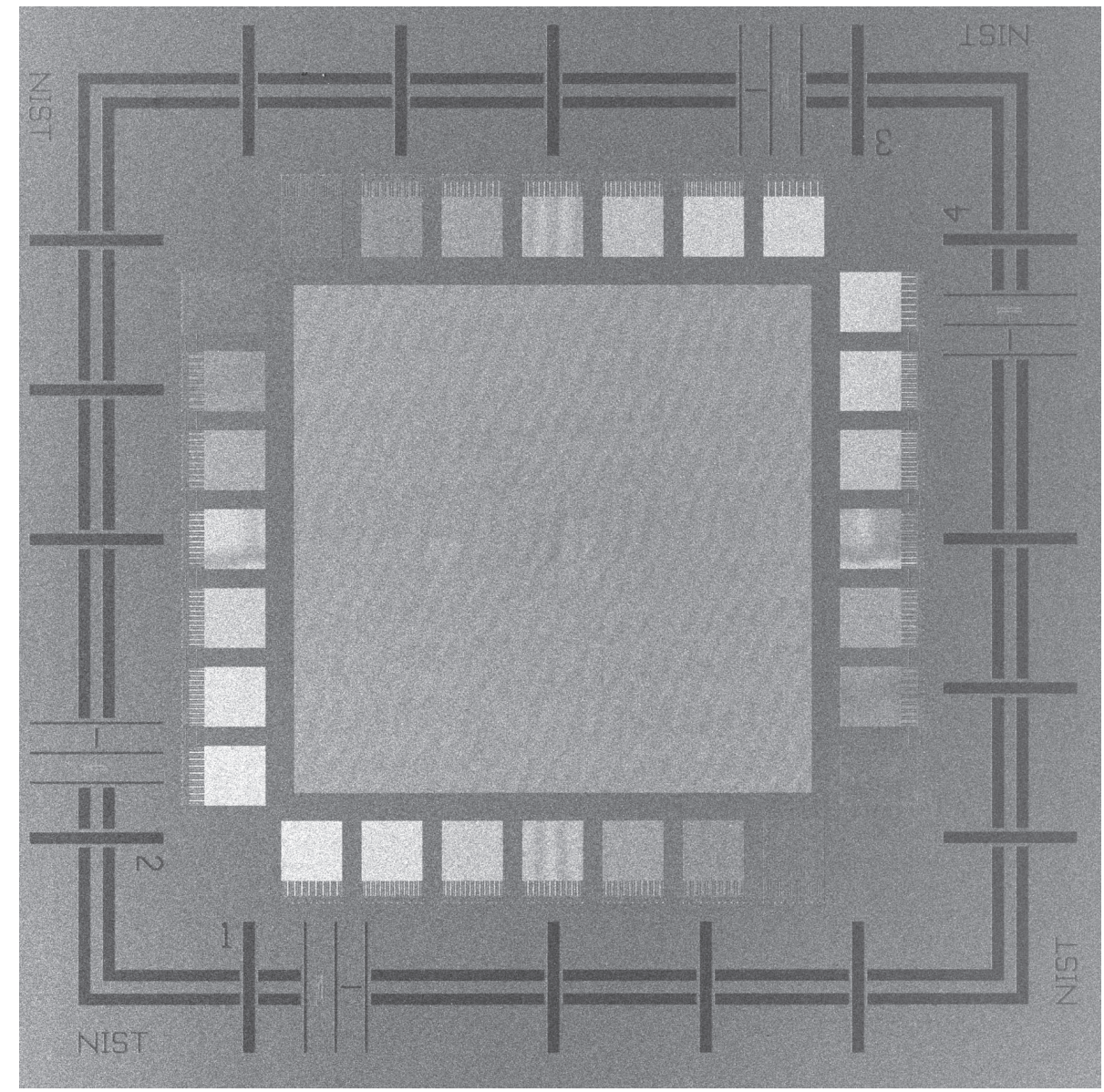

Figure 8. SEM micrograph of the complete RM 8820 structure. The pitch values of the large square frame structure are $1.5 \mathrm{~mm}$ in both $X$ and $Y$ directions as shown in Figure 4. (HFW $=1.75 \mathrm{~mm})$ 


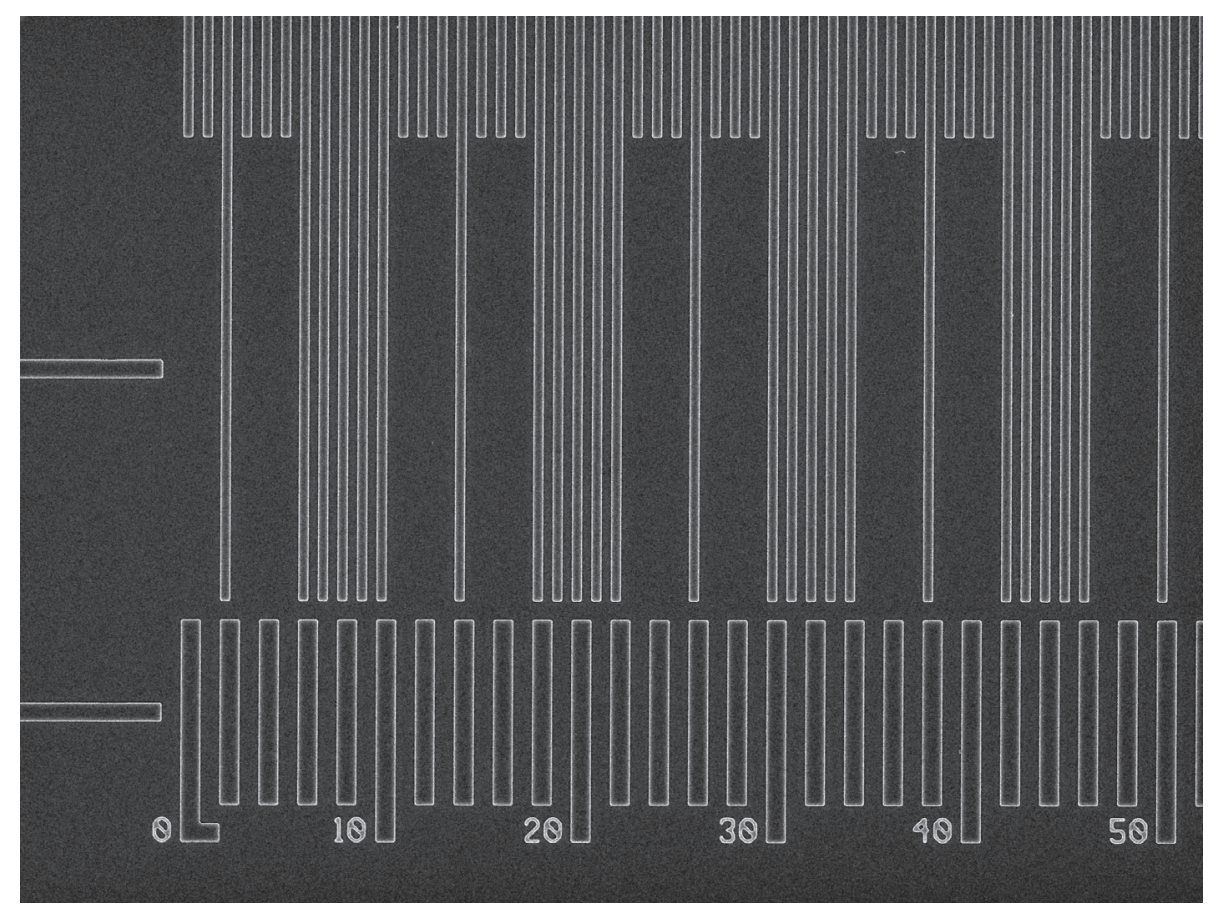

Figure 9. Typical low-magnification image of a roughly $50 \mu \mathrm{m}$ portion of the binary-type pitch structure. The $2 \mu \mathrm{m}$ pitch-numbered guiding lines at the bottom and the isolated and dense structures are clearly visible. (HFW $=60 \mu \mathrm{m})$

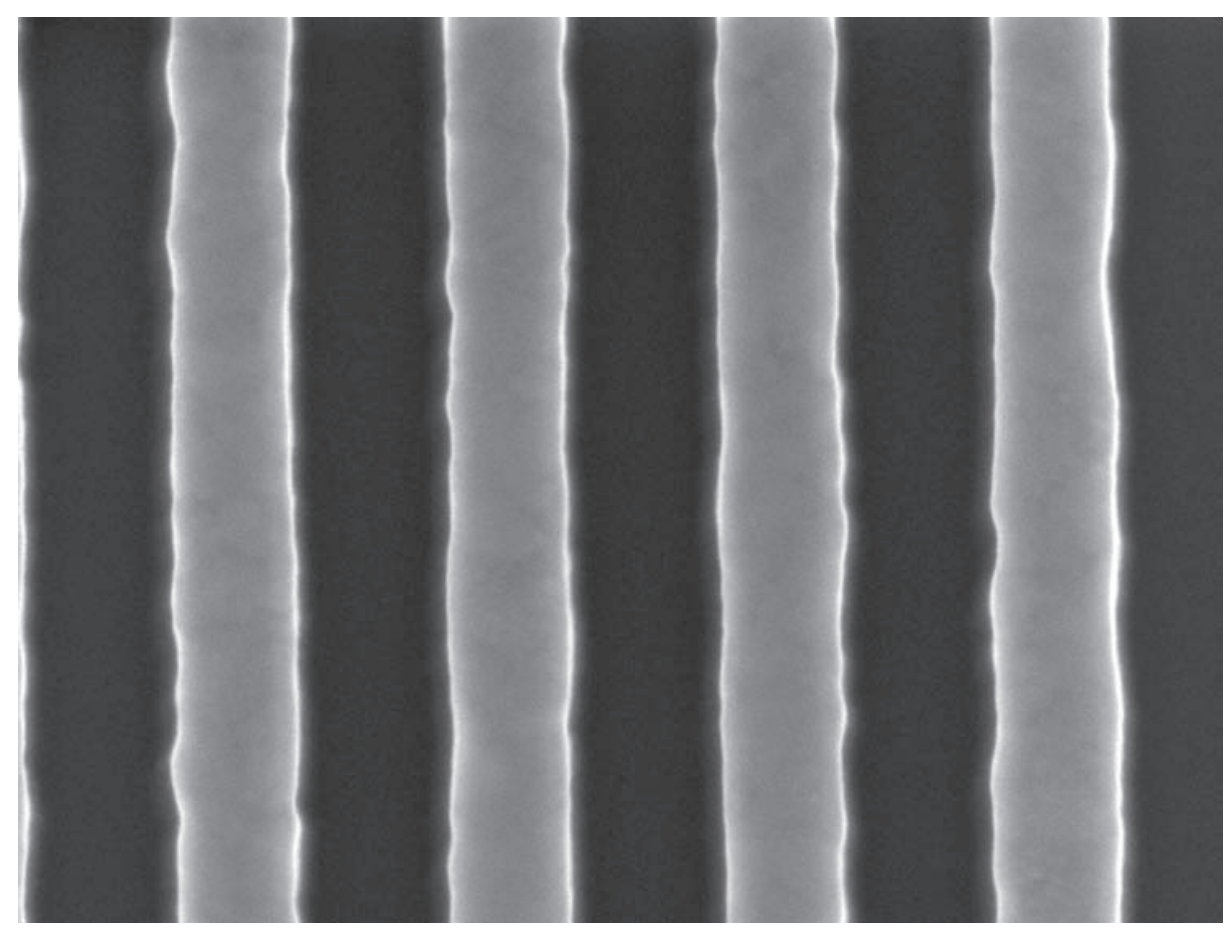

Figure 10. Typical SEM image of the $200 \mathrm{~nm}$ pitch patterns at 100000 times magnification. $(\mathrm{HFW}=813 \mathrm{~nm})$ 


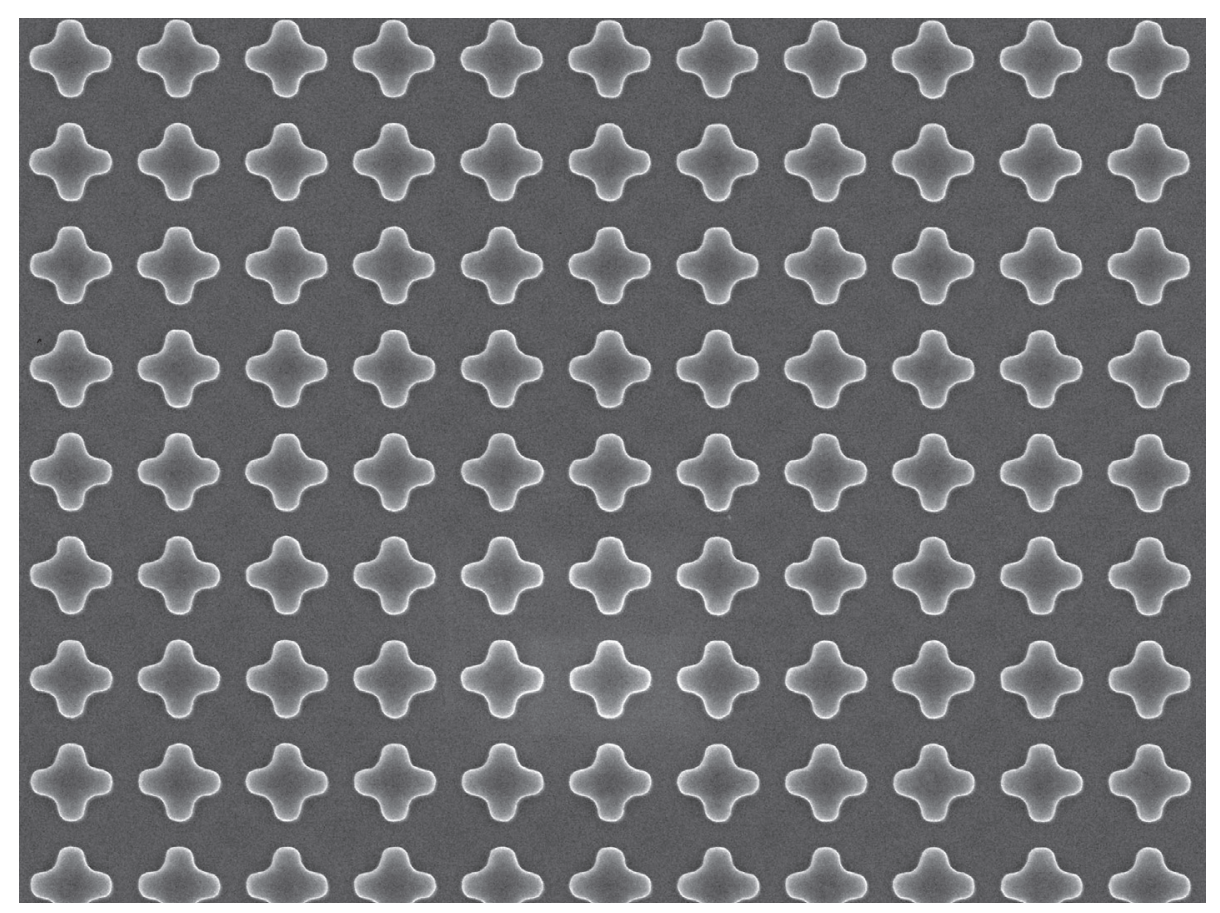

Figure 11. The SEM images of the center cross structures of RM 8820. The pitch values of the structures are $1 \mu \mathrm{m}$ in both $\mathrm{X}$ and Y directions. $(\mathrm{HFW}=12 \mu \mathrm{m})$

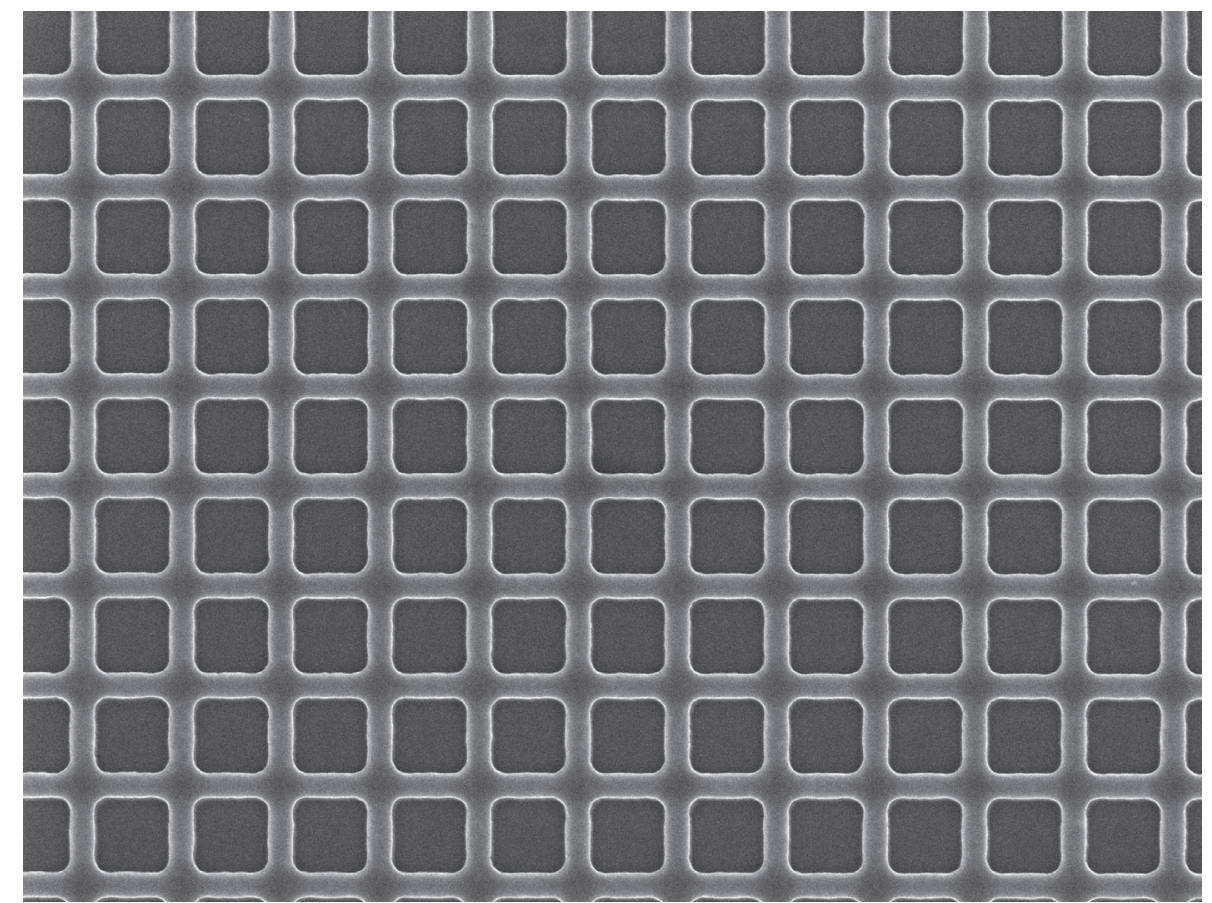

Figure 12. The SEM images of the center grid structures of RM 8820 . The pitch values of the structures are $1 \mu \mathrm{m}$ in both $\mathrm{X}$ and Y directions. (HFW $=12 \mu \mathrm{m})$ 


\begin{tabular}{|c|c|c|c|}
\hline \multicolumn{4}{|c|}{$\begin{array}{c}\text { Table } 1 \\
\text { RM } 8820 \text { Measurements }\end{array}$} \\
\hline Pitches & Nominal $(\mu \mathrm{m})$ & Metrology Instrument $(\mu \mathrm{m})$ & Inspection Instrument (nm) \\
\hline 1 & 1500 & 1500.1 & $\mathbf{n} / \mathbf{a}$ \\
\hline 2 & 1000 & 1000.1 & $\mathbf{n} / \mathbf{a}$ \\
\hline 3 & 500 & 500.2 & $\mathbf{n} / \mathbf{a}$ \\
\hline 4 & 250 & 250.3 & $\mathbf{n} / \mathbf{a}$ \\
\hline 5 & 100 & 100.2 & $\mathbf{n} / \mathbf{a}$ \\
\hline 6 & 50 & 50.1 & $\mathbf{n} / \mathbf{a}$ \\
\hline 7 & 10 & 10.0 & $\mathbf{n} / \mathbf{a}$ \\
\hline 8 & 4 & 4.01 & $\mathbf{n} / \mathbf{a}$ \\
\hline 9 & 1.4 & 1.4 & $\mathbf{n} / \mathbf{a}$ \\
\hline 10 & 2 & 2.0 & $\mathbf{n} / \mathbf{a}$ \\
\hline 11 & 1 & 1.0 & $\mathbf{n} / \mathbf{a}$ \\
\hline 12 & 0.7 & 0.70 & $\mathbf{n} / \mathbf{a}$ \\
\hline 13 & 0.5 & 0.50 & $\mathbf{n} / \mathbf{a}$ \\
\hline 14 & 0.4 & 0.40 & 400.7 STD 1.2 \\
\hline 15 & 0.28 & 0.28 & $\mathbf{n} / \mathbf{a}$ \\
\hline 16 & 0.2 & 0.20 & 199.6 STD 0.9 \\
\hline
\end{tabular}

useful if the instrument in use is expected to contaminate the RM sample structures.

Figure 5 shows the design and the nominal values for the $\mathrm{X}$ and $\mathrm{Y}$ direction large pitch structures of one of the nonconnected RM 8820 patterns. The two sets of patterns are designed to be identical in both of the $\mathrm{X}$ and $\mathrm{Y}$ pitch directions. These are marked with numbers 1 and 3 for the $\mathrm{X}$ direction and 2 and 4 for the $\mathrm{Y}$ direction. All structures were designed to be either parallel with or perpendicular to each other. Between the $50 \mu \mathrm{m}$ pitch patterns are much smaller pitch patterns, shown at the right side of the drawing of Figure 4.

The finest calibration structures are the $200 \mathrm{~nm}$ pitch structures found in the center of the $4 \mu \mathrm{m}$ pitch structures (schematically shown on the right side of Figure 5) lithographically they have merged into one larger structure. This has occurred because the resolution of the optical lithography technique for that area was not sufficient to resolve these small pitch structures as binary mask features. The structures marked A to G on Figure 6 were designed to solve this problem and provide $200 \mathrm{~nm}$ to $2 \mu \mathrm{m}$ pitches. The A $200 \mathrm{~nm}$ and B $280 \mathrm{~nm}$ pitch structures were designed and fabricated with phase shifting patterns. Therefore, they have been resolved in this location. These are useful for pitch calibration up to about $300000 \mathrm{x}$ magnification. But, as a minor consequence of the phase shifting lithography method, these are not fabricated as equal lines and spaces; the widths of the lines are smaller than the widths of the spaces between them. The C $400 \mathrm{~nm}$, D $500 \mathrm{~nm}, \mathrm{E} 700 \mathrm{~nm}, \mathrm{~F} 1 \mu \mathrm{m}$ and G $2 \mu \mathrm{m}$ pitch calibration structures were made with the binary mask and were fabricated as relatively equal line widths and space widths. All A to $\mathrm{G}$ pitch calibration structures were made as dense lines of large $(100 \mu \mathrm{m}$ by $120 \mu \mathrm{m})$ areas with the same pitch, and there are dense and isolated lines that extend beyond the dense calibration areas. These were designed to facilitate cross sectional measurements of dense and isolated lines after precision cleaving. All small structures at their bottom also have a $1 \mu \mathrm{m}$ line, $1 \mu \mathrm{m}$ space structure and 0,10 , etc. numbers up to 100 to help the identification of exact locations within the small patterns themselves. After locating one of these numbers, one can easily and with very little sample motion arrive at isolated or dense lines or somewhere in the $100 \mu \mathrm{m}$ by $120 \mu \mathrm{m}$ areas. This, generally, may be done without any focus adjustment if the sample is mounted normal (perpendicular) to the beam. All patterns with the same designation were designed to be identical, e.g., the four A patterns (two in the X direction and two in the $\mathrm{Y}$ direction) are the same design. Figures 6 and 7 show the schematic design of the location of the A through 


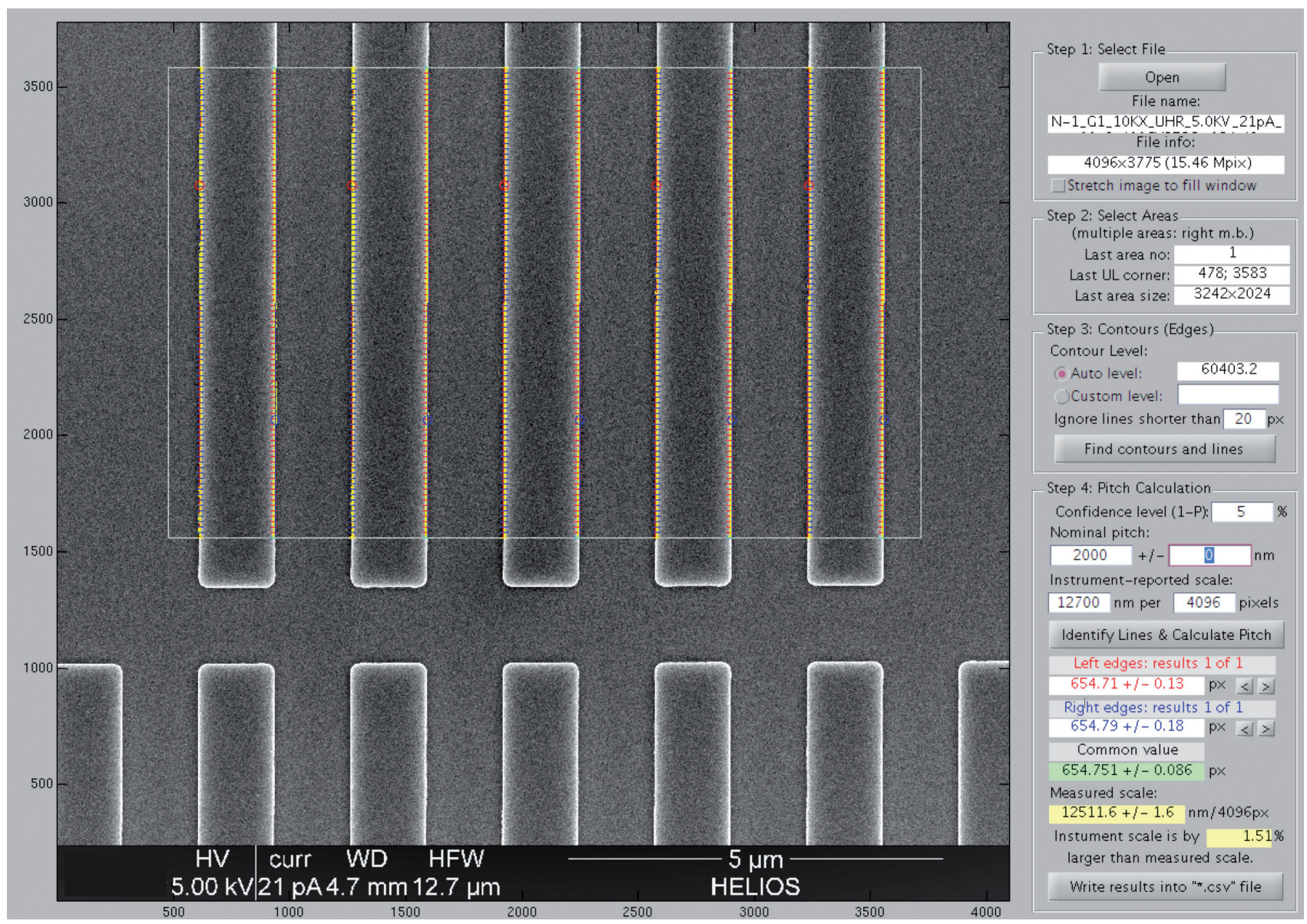

Figure 13. A typical screen shot of the NIST Pitch Calibration software program available fo use with RM 8020.

G structures. All other structures have similar designs, but with different pitch values (as described above).

3.3 SEM Imaging. A SEM image of the entire RM 8820 structure, at low magnification, is shown in Figure 8 . The pitch values of the large square frame structure are $1.5 \mathrm{~mm}$ in both $\mathrm{X}$ and $\mathrm{Y}$ directions. Figure 9 shows a typical low magnification image of a roughly $50 \mu \mathrm{m}$ portion of a binary type, $1 \mu \mathrm{m}$ pitch structure. The $2 \mu \mathrm{m}$ pitch numbered guidelines at the bottom and the isolated and dense structures are clearly visible. Figure 9 shows a typical SEM image of the $200 \mathrm{~nm}$ pitch patterns at a HFW of about $813 \mathrm{~nm}$ or about 100000 times magnification (Figure 10). Crosses (Figure 11) and grids (Figure 12) are found in the center of the RM 8820 pattern which are used for focus and astigmatism correction as well as scan linearity measurements.

3.4 Reference Scale Calibration Values. The reference values for a large set of scale calibration values are provided in Table 1. The reference values were determined by combined SEMATECH CD-SEM and laboratory SEM measurements. The reference values supplied are non-certified values that are the best estimate of the true value. These values do not meet all of the NIST criteria for Standard Reference Material certification, but do provide a reference value. The values are provided with large associated uncertainties since not all of the sources of uncertainty for the Reference Material have been fully explored.

3.5 NIST Pitch Calibration Software Program. A public domain pitch calculation software program is available from NIST, which calculates the pitch on hundreds or thousands of locations and gives a statistical measure of the pitch values (Figure 13). The program uses a robust and comprehensive algorithm for measuring pitch in images of the calibration 


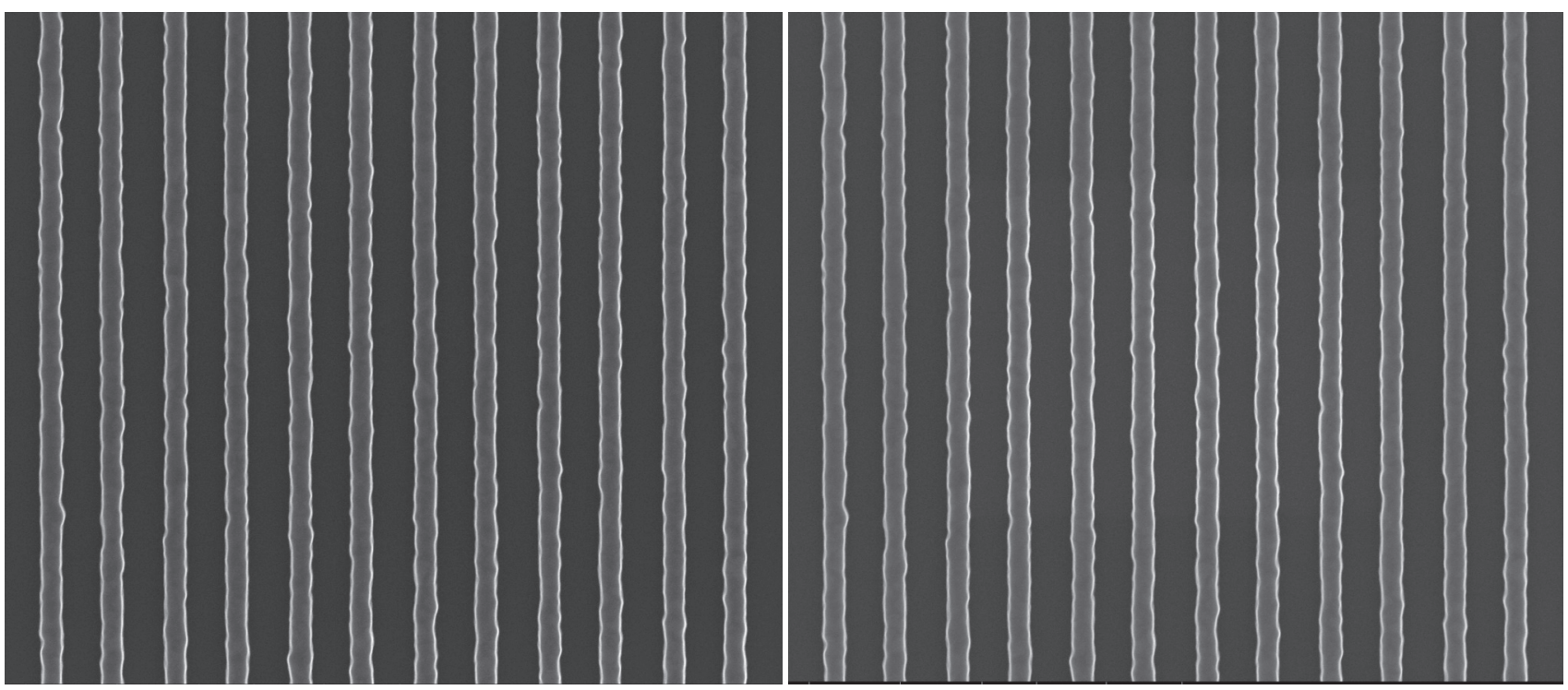

Figure 14. Contamination free SEM operation at high accelerating voltage. $15 \mathrm{kV}, 86 \mathrm{pA}$ SEM images of amorphous Si patterns at the beginning of the test (left) and after 10 minutes (right of continuous electron beam bombardment at twice as high magnification. Essentially no contamination is observable. (HFW $=2.56 \mu \mathrm{m})$
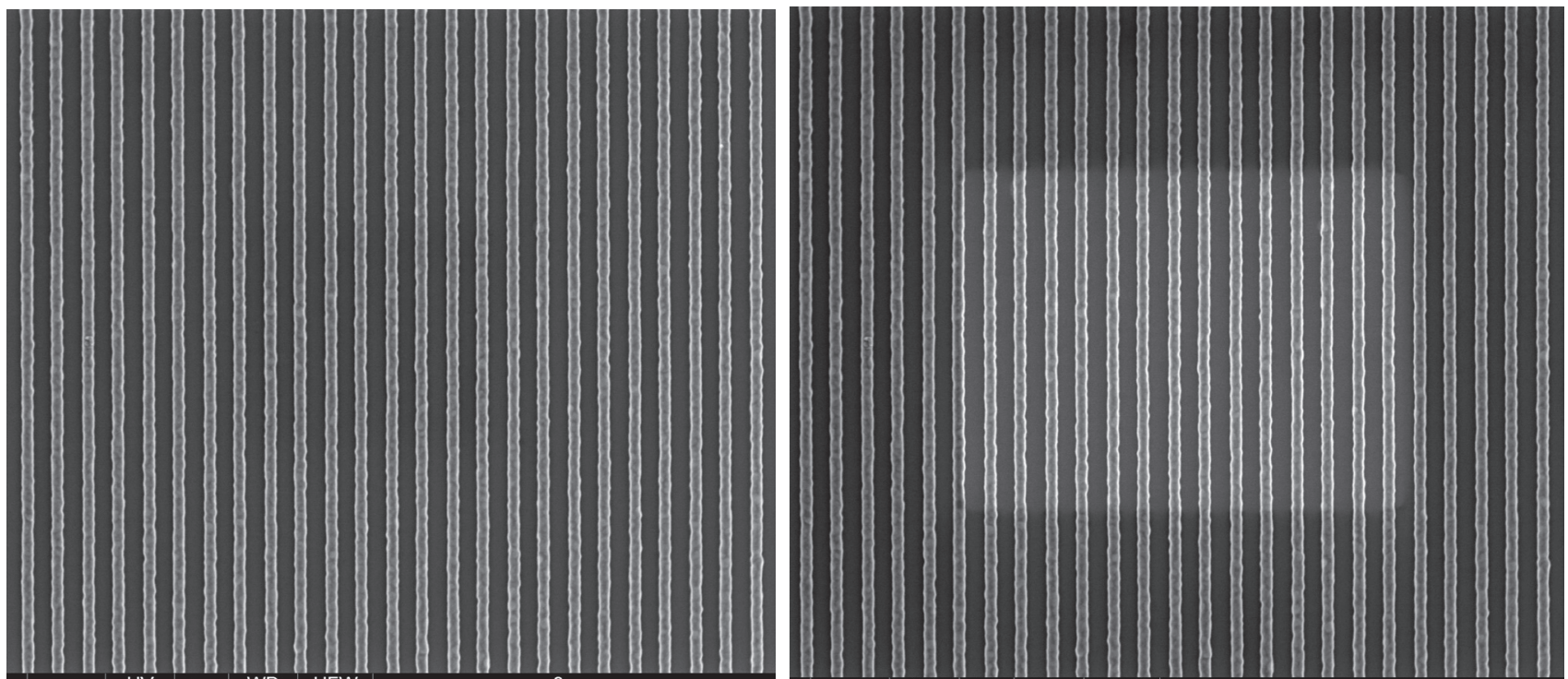

Figure 15. Contamination free SEM operation at low accelerating voltages. $1 \mathrm{kV}, 86$ pA SEM images of amorphous Si patterns at the beginning of the test (left) and after 10 minutes (left) of continuous electro beam bombardment at twice as high magnification. Actual cleaning and enhancement of secondary electron emission is observable. $(\mathrm{HFW}=5.12 \mu \mathrm{m})$ 
samples. The algorithm was implemented and tested in a Matlab program. The algorithm requires a few parameters to be set before processing: contour level, angular acceptance and confidence level for the periodic pattern matching. However, for most images, the default values found automatically or preset in the program work well. No or little user intervention is therefore needed. This software program was developed by Martin Oral of NIST and is currently available through the NIST website (https://www-s.nist.gov/srmors/view_detail.cfm?srm=8820) or by contacting Andras Vladar (andras. vladar@nist.gov). This program is provided as an aid to the RM users and is not guaranteed to function on all computer operating systems, but only on those on which it has been tested.

3.6 Contamination-Free Measurements. Electron beam-induced sample contamination is a serious but avoidable problem associated with SEM imaging and metrology. Even a thin carbonaceous layer can significantly and unacceptably alter the sample. The sample surface is never completely clean, because typically a thin layer of molecules containing water and carbon dioxide is on the surface of samples that have been exposed to room air. Other possible contaminant molecules could come from storage containers or from the fabrication processes, the vacuum in the specimen chamber or outgassing from various parts of the sample stage - any species that have high mobility and long mean free paths in vacuum conditions. Any or all of these contamination sources can end up in the vicinity of the region hit by the electron beam. In a dynamic process of adsorption and desorption, these molecules can be deposited on the surface of the sample. The number of these molecules can be very large, so much so, that it literally makes the sample features grow larger. Beyond this, the contamination process changes the very top surface of the original sample to a carbonaceous-like material, which generally leads to a significant drop in the secondary electron emission. Both of these phenomena have detrimental effects. First, the sample changes its size, which is a critical parameter (especially for IC process control), and second, the smaller electron emission leads to unpredictable results. This is a consequence of the fact that the measurement is reporting results not only on the sample but on the sample with an uncharacterized layer of different material on the top of it. For accurate dimensional measurements and adequate process control, it is essential to minimize this electron beam induced contamination.

It is possible to achieve essentially contamination-free SEM operation [10-13]. Figure 14 (left and right) are images taken at $15 \mathrm{kV}$ accelerating voltage, and Figure 15 (left and right) are images taken at $1 \mathrm{kV}$ accelerating voltages showing the results of a clean operating dimensional metrology SEM. After 10 minutes of continued electron beam bombardment of the center portion of the sample, there is no visible contamination; actually sample cleaning is observable, and is especially pronounced in the low energy case (Figure 15). The derivation and control of the enhancement of the secondary electron signal is an area currently under research.

Regular monitoring of the contamination performance of the SEM is critical. To separate sample-related and instrumentrelated contamination and thus avoid misleading results, it is necessary to have a clean sample, or a sample that can be cleaned. It was found that a sample such as RM 8820 or an amorphous Si structures on $2 \mathrm{~nm}$ gate oxide on Si substrate work well for testing charged particle beam induced contamination. Wet chemical solution and thorough rinsing were found to regenerate the cleanliness of the RM when necessary [10 - 13].

If the instrument fails the cleanliness test with a known clean sample, the instrument needs cleaning. A low-energy oxygen plasma cleaning process has been used successfully [11]. It can very effectively eliminate oily residues from the vacuum and from the surfaces within the sample chamber, including the surfaces of the sample stage. It is important to point out that the ionized oxygen generated by the plasma cleaner oxidizes many materials, but, advantageously, the process is especially effective on hydrocarbon residues. It is recommended that a minimum, but sufficient time and plasma power ( $5 \mathrm{~W}$ to $7 \mathrm{~W}$ ) be employed. Manufacturer recommendations for the operation of the plasma cleaner should be closely followed and the application of the plasma cleaning process should also be discussed with the particle beam instrument manufacturer before initial use.

Unnecessary cleaning is not recommended. If the instrument meets the contamination specification, there is no need for cleaning. It takes some time to go through the cleaning process, which involves (in one method) leaking in air and keeping the sample chamber at around $60 \mathrm{~Pa}$ pressure for the time of the plasma cleaning and then pumping it back down. Excessive exposure of the sample chamber and sample stage to oxygen plasma might result in undesirable consequences and, as stated above, such procedures should always be discussed with the instrument manufacturer prior to use. 


\subsection{Additional Patterns Included on RM 8090}

NIST RM 8820 contains many lithographically printed patterns from a number of sources generated through the SEMATECH AMAG and OMAG cooperation. These patterns are shown and described in the Supplementary Patterns section as Appendix 1. Also included on this chip is the AMAG/OMAG pattern set used for printing SRM 2059 and SRM 2089. These are to be considered just structures to be used and are not replacements for the SRMs with a similar number identifier. The RM 8820 pattern is the only pattern primarily traceable to NIST on this chip. Secondary traceability can be established by the user using any of the structures.

\subsection{Conclusion}

NIST RM 8820 were developed under the strong collaboration between the NIST and SEMATECH. These standards were designed through the cooperation with the SEMATECH AMAG and the OMAG and was fabricated at SEMATECH using current advanced lithography techniques. RM 8820 wwere designed to be as versatile as possible and to utilize all the available the real estate on the silicon chip as efficiently as possible. The numerous test patterns are useful for many different instruments and can be used to calibrate the entire suite of microscopes in most laboratories. Advanced lithography techniques continue to improve and the next step is a version based upon the AMAG 6 reticle. It is anticipated that this artifact will be released in both an RM and an SRM form sometime in the near future.

\subsection{Acknowledgements}

The authors would like to acknowledge and thank SEMATECH and all the SEMATECH AMAG and OMAG members who, over the years, contributed to the design of the overall AMAG 5 pattern and both Drs. Jack Martinez and John Kramar at NIST for their early reviews of this publication.

\subsection{References}

[1] Boyde, A. 1979. Why horizontal field width? (Editorial Comment) SCANNING 2:126-128.

[2] Postek, M. T. and Tiberio, R. C. 1988. Low Accelerating Voltage SEM Magnification Standard Prototype. EMSA Proceedings pp. 198-199.

[3] Postek, M. T. and Gettings, R. 1995. Report of Investigation: Reference Material 8090 - SEM Magnification Calibration Reference Material. OSRMP 6 pp.

[4] Postek, M. T. 1989. Scanning Electron Microscope based Metrological Electron Microscope System and New Prototype SEM Magnification Standard. Scanning Microscopy 3(4): 1087-1099.

[5] Postek, M. T. and Tiberio R. C. 1990. Submicrometer SEM Magnification Standard. National Nanofabrication Facility Research Accomplishments 1988 1989. Unpaged.

[6] Newell, B. L., Postek, M. T. and van der Ziel, J. P. 1995a Performance of the NIST SRM 2090A SEM magnification calibration standard in a low voltage SEM. Proceedings SPIE 2439: 383-390.

[7] Newell, B. L., Postek, M. T. and van der Ziel, J. P. 1995b. Fabrication issues for the prototype NIST SRM-2090A SEM magnification calibration standard. J. Vac. Science and Technology B 13 (6): 2671-2675.

[8] Newell, B. L., Postek, M. T. and van der Ziel, J. P. 1995c. Fabrication issues for the prototype NIST SRM-2090A SEM magnification calibration standard. Proceedings EIPB p 113-114.

[9] Postek, M. T. , Vladar, A. E. , Jones, S. and Keery, W. J. 1993. Inter-laboratory study on the lithographically produced scanning electron microscope magnification standard prototype. NIST J. Res. 98:447-467. 
[10] Vladar, A. E. , Purushotham, K. P. , and Postek, M. T. 2008. Contamination Specification for Dimensional Metrology SEMs. Proc. SPIE 6922: 692217-1 - 692217-5.

[11] Vladar, A. E., Postek, M. T. and Vane R. 2001. Active monitoring and control of electron-beam-induced contamination. SPIE 4344:835-843.

[12] Postek, M. T., Vladár, A. E., Does Your SEM Really Tell the Truth?-How Would You Know? Part 2. Specimen Contamination. SPIE SCANNING Microscopy 2013, SPIE Proceedings 8729:872903 1-6.

[13] Postek, M. T., Vladar, A. E. 2013. Does Your SEM Really Tell the Truth? How would you know? Part 1. SCANNING 35:355-361. 
Page left intentionally blank 


\section{$\underline{\text { Appendix } 1}$}

\section{Supplemental Patterns}

The NIST RM 8820 calibration pattern was described in the previous sections. The Supplemental Patterns section describes the numerous additional patterns included on the semiconductor chip in addition to the RM 8820 patterns. These patterns can be used for a whole host of purposes including as: secondary calibration structures, stage or scan linearity test structures, etc. RM 8820 and these additional structures can be used to calibrate all microscopes (optical, SEM, AFM, etc.) in a laboratory to the same standard.

Several copies of some of these additional patterns are printed across the chip. They have only been described once generally upon their first occurrence.

Attempts have been made to provide pertinent wide view (low magnification) drawings, as well as, complimentary more detailed narrow field (higher magnification) drawings. Because of the limited resolution of the transfer process, some of the finer detail is unfortunately lost from the drawings. As many of the guiding features as possible have been retained in the examples used. In addition, scanning electron micrographs are included to aid in clarification of many of the patterns. Please note that several copies of some of these additional patterns are printed across the chip. They have only been described once, generally upon their first occurrence.

Please note that these accessory patterns are included as an addition to the RM 8820 pattern and in some cases these additional patterns may not have printed correctly.

\section{Acronyms:}

CAD - Computer Aided Design

CD - Critical Dimension

FOV - Field of View - typically applies to the CAD or GDSII figures

GDSII - Graphic Database System II

HFW - Horizontal Field Width - typicall applies to SEM images (see: page 7)

DCP - Dimensional Control Pattern 
Page left intentionally blank 


\section{Entire RM 8820 Test Pattern}


Plate 1. Entire RM 8820 calibration standard. Low magnification optical micrograph of the NIST patterns and the entire array of additional patterns supplied on RM 8820. For descriptive purposes, this overall chip has been broken-up into 7 sections. The subsequent pages describe these 7 sections and the included patterns within those areas. Many patterns are duplicated across the chip, but they are only described once.

Please note that the horizontal field width of the scanning electron micrographs are noted in the alphanumerics of the micrograph and are not in the written description, in all cases. 


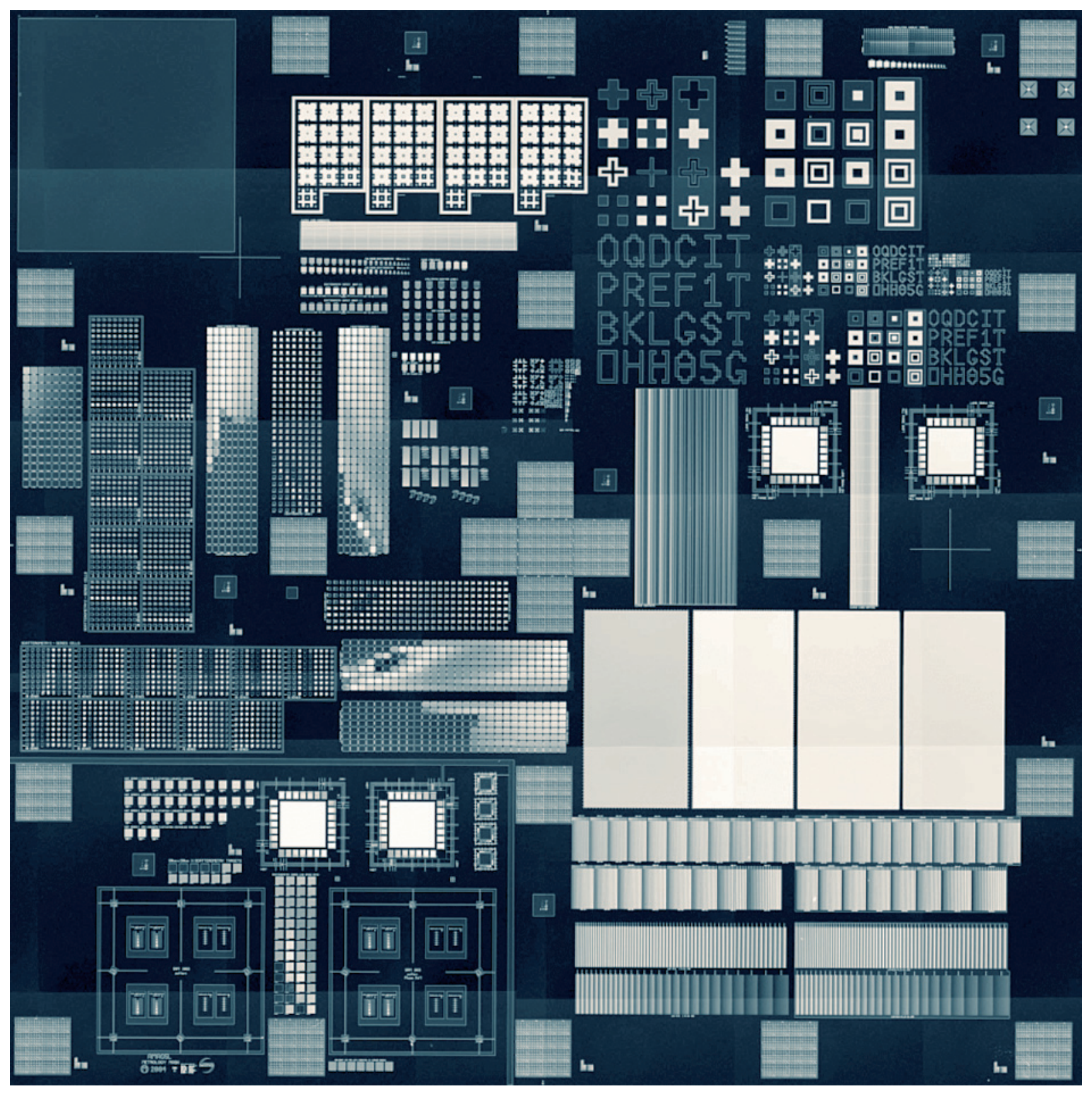




\section{Description of Patterns Section 1}

Plate 1. Entire RM 8820 calibration standard. Low magnification optical micrograph of the NIST patterns and the entire array of additional patterns supplied on RM 8820. For descriptive purposes, this overall chip has been broken-up into 7 sections. The subsequent pages describe these 7 sections and the included patterns within those areas. Many patterns are duplicated across the chip, but they are only described once.

Please note that the horizontal field width of the scanning electron micrographs are noted in the alphanumerics of the micrograph and are not in the written description, in all cases.

Plate 2. Section 1-0 - Overall. (top) GDS II line drawing of the entire RM 8820 pattern highlighting Section 1 with the 8 additional major sub-patterns numerically identified. (bottom) GDS II detail drawing of Section 1 showing the 8 additional major sub-patterns included in this section.

Plate 3 - Pattern 1-1 - Critical Dimension Measurement (CD). (top) GDS II drawing of critical dimension Pattern 1-1 at low magnification. The smaller Pattern 1-2 is also represented in this view. (bottom) SEM micrograph of Pattern 1-1 and 1-2 in the same field. Pattern 1-1 is replicated 25 times across the chip. (But, these patterns are only described once at the first occurrence noted).

Explanation:

CD measurement features. Dimensional Control Patterns (DCP) are useful for critical dimension measurement testing in metrology instruments. Each built-in pattern recognition structure includes: Lines: 1:1, 2:1 \& 3:1; Isolated (Iso) space; Contact Holes 1:1, 2:1, \& 4:1. Contact Holes only exist in the $80 \mathrm{~nm}$ cells and above due to tool limitations. This feature was designed with several important factors in mind: a) to maximize robustness of the smallest of the features for ensuring that testing would be valid to the smallest possible nodes; b) to ensure a wide variety of feature sizes for linearity testing; c) for extremely simple, intuitive navigation; d) availability of features in both horizontal and vertical directions; e) many repetitions across the scanner field, allowing across-field variation to be measured if necessary; and f) many redundant, identical copies of the same features to allow for repetition of different experiments that involve sample damage by SEM, such as with contamination or photoresist shrinkage (the key concept could be described as many "disposable features" within each die).

Pattern 1.1 represents the SEMATECH version of what many in the industry describe as "L-bars". The $5 \times 5$ array shown on the left is exactly $250 \mu \mathrm{m}$ square, and a $4 \times 4$ block of these always were placed together to make $1 \mathrm{~mm}$ square modules at 25 different locations across the field; thus there are 400 copies of each of the features available on each die. On the left is a close-up of a 50 um square cell within the 5x5 matrix; note that each is carefully marked with nominal size on reticle, and includes vertical and horizontal lines, contact holes, isolated trenches and a few different line/space pitches from dense to isolated, and that robust pattern recognition and feature anchors are also available. 
Plate 4 - Pattern 1-1 - Critical Dimension Measurement (CD). (top) GDS II drawing of critical dimension Pattern 1-1 at a higher field of view. (bottom) SEM micrograph of a similar area.

Plate 5 - Pattern 1-1 - Critical Dimension Measurement (CD). (top) GDS II drawing of critical dimension Pattern 1-1 at a higher magnification. (bottom) SEM micrograph of a similar area.

Plate 6 - Pattern 1-2 - Critical Dimension Measurement (CD). (top) GDS II drawing of critical dimension Pattern 1-2. (bottom) SEM micrograph of a similar area.

Plate 7 - Pattern 1-2 - Critical Dimension Measurement (CD).(top) GDS II drawing of critical dimension Pattern 1-2 at a higher magnification. (bottom) SEM micrograph of a similar area.

Plate 8 - Pattern 1-3 - SRM 2059 pattern. (top). GDS II drawing of Pattern 1-3 at low field of view. (bottom) SEM of a corner of the pattern.

Explanation:

NIST Pattern for SRM 2059. This pattern is a printing of the NIST SRM 2059 photomask optical microscope linewidth calibration standard. This is not meant to be a replacement to this standard. This is an area that combines various line and space scatterometry cells located at the center with the metrology structures of the existing SRM 2059 photomask linewidth standard (NIST SRM 2059). This area was originally designed by James Potzick of NIST. [see the NIST website for further description]

Plate 9 - Pattern 1-3 - SRM 2059 pattern. (top) GDS II drawing of Pattern 1-3 at low field of view. (bottom) SEM of a similar field of view.

Plate 10 - Pattern 1-3 - SRM 2059 pattern. (top) GDS II drawing of one of the smaller patterns of 1-3. (bottom) SEM of a reduced field of view of some of the calibration features of SRM 2059 showing multiple pitch lines.

Plate 11 - Pattern 1-4 - Scatterometry Target. (top) GDS II drawing of the $150 \mu \mathrm{m}$ by $150 \mu \mathrm{m}$ scatterometry targets. (bottom) SEM of a similar area of the RM 8820 reference material.

Explanation:

Scatterometry Target. The scatterometry target uses the $150 \mathrm{~nm}$ 1:1 trenches over different areas. The following is a list of the different areas covered by the $140 \mathrm{~nm}$ trench: 100x100 $\mathrm{m}, 90 \times 90 \mu \mathrm{m}, 80 \times 80$ $\mu \mathrm{m}, 70 \times 70 \mu \mathrm{m}, 60 \times 60 \mu \mathrm{m}, 50 \times 50 \mu \mathrm{m}, 40 \times 40 \mu \mathrm{m}, 30 \times 30 \mu \mathrm{m}, 20 \times 20 \mu \mathrm{m}$, and $10 \times 10 \mu \mathrm{m}$.

Plate 12 - Pattern 1-4 - Scatterometry Target. (top) GDS II drawing of one of the smaller patterns of 1-4 at a reduced FOV. (bottom) SEM micrograph of a similar area. 
Plate 13 - Pattern 1-4 - Scatterometry Target. (top) GDS II drawing of one of the $150 \times 150 \mu \mathrm{m}$ patterns of 1-4 at a reduced FOV. (bottom) SEM micrograph of a similar area.

Plate 14 - Pattern 1-5 - Line edge roughness. (top) GDS II drawing of Pattern 1-5 at a wide field of view. (bottom) SEM Micrograph of a similar area.

\section{Explanation:}

Line Edge Roughness (LERNIST). NIST designed line space arrays with built in periodic line edge roughness (LER). The amplitude and the frequency (wavelength) of the intentional edge roughness pattern is varied across a set of parameters. The values noted are the nominal linewidth and roughness periodicity, design values, which after the IC fabrication process changes somewhat. Some of the intentional changes fade into the "noise" caused by other characteristic process and materialdependent effects. For example, depending on the grain size of the polysilicon or properties of the photoresist and exposure/develop process, there is an unavoidable random roughness of all lines. Some of the lines have intentional edge only on one side, others might have other variations. These are depicted with identifiers and is well visible on SEM images. These are designed in 150 um square pads with $100 \mu \mathrm{m}$ space, ideal for OCD or CDSAXS experiments on such periodic roughness. Tabs are 1/2 of periodicity.

Series 4 - 6: L100P300: Amp 5-35 (5 nm steps) / Per 110; L150P450: Amp 20, 40, 60 / Per 200

Series 7: L150P450: Amp 20, 40, 60 / Per 200

Series 4 has alternating tabs on left/right side of line

Series 5 has double aligned tabs on left/right side of line

Series 6 has alternating tabs on left/right side of line; but lines are staggered

Series 7 has tabs only on one side

Plate 15 - Pattern 1-5 - Line edge roughness. (top) GDS II drawing of Pattern 1-5 at a smaller field of view. (bottom) SEM micrograph of a similar area.

Plate 16 - Pattern 1-5 - Line edge roughness. (top) Medium magnification SEM image of Pattern 1-5. (bottom) Smaller HFW SEM micrograph of a similar area.

Plate 17 - Pattern 1-6 - Scatterometry Varied. (top) GDS II drawing of Pattern 1-6 at a wide field of view. (bottom) SEM micrograph a similar area..

Explanation:

Scatterometry Target. The scatterometry target uses the $150 \mathrm{~nm}$ 1:1 trenches over different areas. The following is a list of the different areas covered by the $140 \mathrm{~nm}$ trench: 100x100 $\mu \mathrm{m}, 90 \times 90 \mu \mathrm{m}, 80 \times 80$ $\mu \mathrm{m}, 70 \times 70 \mu \mathrm{m}, 60 \times 60 \mu \mathrm{m}, 50 \times 50 \mu \mathrm{m}, 40 \times 40 \mu \mathrm{m}, 30 \times 30 \mu \mathrm{m}, 20 \times 20 \mu \mathrm{m}$, and 10x10 $\mathrm{mm}$.

Plate 18 - Pattern 1-6 - Scatterometry Varied. (top) GDS II drawing of Pattern 1-6 at a wide field of view. (bottom) SEM micrograph a similar area of Pattern 1-6. 
Plate 19 - Pattern 1-6 - Scatterometry Varied. (top) GDS II drawing of Pattern 1-6 at a wide field of view. (bottom) SEM micrograph a similar area of Pattern 1-6.

Plate 20 - Pattern 1-6 - Scatterometry Varied. (top) Higher magnification SEM micrograph of Pattern 1-6 at a low horizontal field width. (bottom) SEM micrograph a similar area of Pattern 1-6 at an even higher magnification and horizontal field of view.

Plate 21 - Pattern 1-7 - Via Pad Variation. (top) GDS II drawing of Pattern 1-7 at a wide field of view. (bottom) SEM Micrograph of a similar area.

\section{Explanation:}

Via Pad Varied Corner Radius. This module contains 150x150 $\mu$ m blocks of vias that vary based on corner rounding. The following sizes indicate the rounded corner radius 5, 10, 15, 20, 25, 30, $35 \mathrm{~nm}$; the pad dimensions are: 100x150 $\mathrm{m}$, Pitch: X: $200 \mu \mathrm{m}, \mathrm{Y}: 300 \mu \mathrm{m}$.

Plate 22 - Pattern 1-7 - Via Pad Variation. (top) GDS II drawing of Pattern 1-7 at a wide field of view. (bottom) SEM Micrograph of a similar area.

Plate 23 - Pattern 1-7 - Via Pad Variation. (top) SEM micrograph taken of $10 \mathrm{~nm}$ corner radius pattern and (bottom) SEM micrograph of $5 \mathrm{~nm}$ of corner radius pattern.

Plate 24 - Pattern 1-8 - RM 8090. (top) GDS II drawing of Pattern 1-8 at a wide FOV. (bottom) GDS II drawing of one of the smaller Patterns of 1-8 at a smaller FOV.

[The RM 8820 patterns found adjacent to this have been discussed earlier]

\section{Explanation:}

NIST pattern for NIST RM 8090. Several RM 8090 structures were added to the RM 8820 reticle. Within this section there are two RM 8090 structures, regardless of size, one is completely phase shifted and the other is not. In the NIST quad section, there are six RM 8090 structures, two are standard phase shift modules, but one is grounded, and the other four are 1/4 the size of the original. Two of the 1/4 size are line/space modules and two are trench modules; one of the line/space modules is grounded. [The original RM 8090 pattern was originally designed by Michael Postek and Andras Vladar and its history is described earlier in the text.]

Plate 25 - Pattern 1-8 - RM 8090/2090. SEM images of Pattern 1-8 at a wide FOV. (bottom) and one of the smaller Patterns of 1-8 at smaller FOV. 


\section{Section 2}

Plate 26. Section 2-0 Overall. (top) GDS II line drawing of the entire RM 8820 pattern highlighting Section 2 with the 6 additional major sub-patterns numerically identified. (bottom) GDS II detail drawing of Section 2 showing the 6 additional major sub-patterns included in this section.

Plate 27 - Pattern 2-1 - Multiple Gap Lines Positive. (top) GDS II drawing of Pattern 2-1 at a wide FOV. (bottom) SEM micrograph of a similar area.

Plate 28 - Pattern 2-1 - Multiple Gap Lines Positive. (top) GDS II drawing of Pattern 2-1 at reduced FOV. (bottom) SEM micrograph of a similar area.

Plate 29 - Pattern 2-1 - Multiple Gap Lines Positive. (top and bottom) SEM Micrographs of Pattern 2-1 at two different horizontal fields of view.

Plate 30 - Pattern 2-2 - Multiple Gap Lines Negative. (top) GDS II drawing of the interface between the positive 2-1 pattern (left side) and the negative 2-2 pattern at a wide FOV. (bottom) SEM image of Pattern 2-2 at reduced HFW.

Plate 31 - Pattern 2-2 - Multiple Gap Lines Negative. (top) GDS II drawing of the negative 2-2 pattern at a narrower FOV. (bottom) SEM image of Pattern 2-2 at a comparable HFW.

Plate 32 - Patterns 2-3, 2-4, 2-5 and 2-6 - Multiple Size Square Structures Section. (top) Wide FOV of GDS II drawing of Patterns 2-3, 2-4 and 2-5. Pattern 2-6 edge is at the extreme right part of the FOV. (bottom) GDS II drawing of Pattern 2-3 and a portion of Pattern 2-4 at reduced FOV. Pattern 2-3 has nominally $10 \mu \mathrm{m}$ square structures and Pattern 2-4 has nominally $5 \mu \mathrm{m}$ square structures.

Plate 33 - Pattern 2-3 - Multiple Size Square Structures Section. (top) Reduced FOV view of GDS II drawing of Pattern 2-3 showing the nominal $10 \mu \mathrm{m}$ structures. (bottom) SEM micrograph of Pattern 2-3.

Plate 34 - Pattern 2-3 - Multiple Size Square Structures Section. (top, bottom) SEM Micrographs of Pattern 2-3 showing the nominal $10 \mu \mathrm{m}$ structures.

Plate 35 - Pattern 2-4 - Multiple Size Square Structures Section. (top) Reduced FOV view of GDS II drawing of Pattern 2-4 showing the nominal $5 \mu \mathrm{m}$ structures. (bottom) SEM micrograph of Pattern 2-4.

Plate 36 - Pattern 2-4, Multiple Size Square Structures Section. (top, bottom) SEM micrographs of Pattern 2-4 showing the nominal $5 \mu \mathrm{m}$ structures.

Plate 37 - Pattern 2-5, Multiple Size Square Structures Section. (top) Reduced FOV view of GDS II 
drawing of Pattern 2-5 showing the nominal $1 \mu \mathrm{m}$ structures. (bottom) SEM micrograph of Pattern 2-5.

Plate 38 - Pattern 2-6 - Multiple Size Square Structures Section. (top) Reduced FOV view of GDS II drawing of Pattern 2-6 showing the nominal 0.5 $\mu \mathrm{m}$. (bottom) SEM micrograph of Pattern 2-6.

Plate 39 - Section 3-0 Overall. (top) GDS II line drawing of the entire RM 8820 pattern highlighting Section 3 with the three additional major sub-patterns numerically identified. (bottom) GDS II detail drawing of Section 3 showing the three additional major sub-patterns. Pattern 3-1 the Global Alignment Test Pattern is large enough to be included in the wide FOV. Pattern 3-1 is the global alignment test pattern (described below).

\section{Explanation:}

Global Alignment Test Patterns. This module is an array of optical global alignment patterns. There are $180 \mu \mathrm{m}$ dense line symbols and solid polysilicon symbols. There are 5 different cells within the Optical GAP module. The cells are based on the following symbol sizes: 45 $\mu \mathrm{m}, 540 \mu \mathrm{m}$. In this module the dense line symbols appear black.

Plate 40 - Pattern 3-1 Global Alignment Test Pattern. (top, bottom) SEM Micrographs of a portion of the Global Alignment Test Pattern section.

Plate 41 - Pattern 3-2 - Cleave lines. (top) GDS II drawing of the cleave lines pattern at wide FOV. The large gaps delimiting the different size and ratio of the lines are apparent in the image. (bottom) SEM micrograph of the cleave lines at a low FOV. The length of these lines is nominally $4 \mathrm{~mm}$.

\section{Explanation:}

Line/space Cleave. This large set of patterns and large area in size was designed for general linespace measurements and process control purposes and specifically for cleaving for cross-sectional SEM. With their $4 \mathrm{~mm}$ long line length, these structures are easily cleavable making it feasible to make top-down and cross-sectional measurements at the same locations. Various pattern recognition marks are also included to allow for navigation of any tool under test and of the cross-section SEM cleave line if using a micro-cleaver. All the main nominal CD sizes in other marks are represented, with several different pitch ratios and also an isolated line and isolated space of these nominal dimensions. This module includes both horizontal and vertical versions.

Plate 42. Pattern 3-2 - Cleave lines. (top) GDS II drawing of the cleave lines pattern at smaller FOV. The large gaps delimiting the different size and ratio of the lines are apparent in the image. (bottom) SEM micrograph of the cleave lines at a small FOV. The length of these lines is nominally $4 \mathrm{~mm}$.

Plate 43 - Pattern 3-3 - Cleave contacts. (top) GDS II drawing of the cleave contacts pattern at wide FOV. The large gaps delimiting the different size and ratio of the contact holes are apparent in the im- 
age. (bottom) SEM micrograph of Pattern 3-3 at wide HFW. The length of these arrays is nominally $4 \mathrm{~mm}$.

Plate 44 - Patterns 3-3 - Cleave contacts. (top) Reduced FOV view of GDS II drawing of Pattern 3-3 showing the nominal $250 \mathrm{~nm}$ contact hole structures with a nominal 1:1 spacing ratio. (bottom, left) SEM micrograph of a similar area. (bottom, right ) SEM micrographs at a reduced FOV.

Plate 45 - Patterns 3-3 - Cleave contacts. (top) Reduced FOV view of GDS II drawing of Pattern 3-3 showing the nominal $250 \mathrm{~nm}$ contact hole structures with a nominal 1:1 spacing ratio. (bottom) SEM micrograph of a similar area.

Plate 46 - Patterns 3-3 - Cleave contacts. Reduced FOV view of GDS II drawing of Pattern 3-3 showing the nominal $0.25 \mu \mathrm{m}$ contact hole structures with a nominal 2:1 spacing ratio. (bottom,) SEM micrograph of a similar area.

Plate 47 - Patterns 3-3 - Cleave contacts. (top) Reduced FOV view of GDS II drawing of Pattern 3-3 showing the nominal $0.25 \mu \mathrm{m}$ contact hole structures with a nominal 3:1 spacing ratio. (bottom) SEM of a similar area.

Plate 48 - Patterns 3-3 - Cleave contacts. (top) Reduced FOV view of GDS II drawing of Pattern 3-3 showing the nominal $0.25 \mu \mathrm{m}$ contact hole structures with a nominal 5:1 spacing ratio. (bottom) SEM of a similar area.

\section{Section 4}

Plate 49 - Section 4-0 overall. (top) GDS II line drawing of the entire RM 8820 pattern highlighting Section 4 with the 4 additional major sub-patterns numerically identified. (bottom) GDS II detail drawing of section 4 showing the 4 additional major sub-patterns included in this section.

Plate 50 - Pattern 4-1 - Scatterometry lines. (top) GDS II drawing of the scatterometry lines Pattern 4-1 at wide FOV. (bottom) SEM micrograph of a similar area.

Plate 51 - Pattern 4-1 - Scatterometry Lines. (top) GDS II drawing of Pattern 4-1 at a smaller FOV showing some of the detail of the fine patterns. (bottom) SEM micrograph of a similar area.

Plate 52 - Pattern 4-1 - Scatterometry Lines. (top) GDS II drawing of the scatterometry lines Pattern 4-1 at smaller FOV showing the variety of line size and line pitches available. (bottom) SEM micrograph of a similar area.

Plate 53 - Pattern 4-1 - Scatterometry Lines. (top) GDS II drawing of Pattern 4-1 at a smaller FOV showing some of the detail of the $250 \mathrm{~nm}$ lines and $750 \mathrm{~nm}$ pitch spacing pattern. (bottom) SEM micrograph of a similar area. 
Plate 54 - Pattern 4-2 - Scatterometry 2 Series Cells. (top) GDS II drawing of the scatterometry lines Pattern 4-2 at wide FOV. (bottom) SEM micrograph of a similar area.

Plate 55 - Pattern 4-2 - Scatterometry 2 series cells. (top) GDS II drawing of Pattern 4-2 at a smaller FOV showing some of the detail of the fine patterns. (bottom) SEM micrograph of a similar area.

Plate 56 - Pattern 4-2 - Scatterometry 2 Series Cells. (top) GDS II drawing of the scatterometry lines Pattern 4-2 at smaller FOV showing the variety of line size and line pitches available. (bottom) SEM micrograph of a similar area.

Plate 57 - Pattern 4-2 - Scatterometry 2 Series Cells. (top) GDS II drawing of the scatterometry lines Pattern 4-2 at smaller FOV showing the variety of line size and line pitches available. (bottom) SEM micrograph of a similar area.

Plate 58 - Pattern 4-3 and 4-4 - Scatterometry Lines and Trenches. (top) GDS II drawing of the scatterometry lines and trenches of Patterns 4-3 and 4-4 at wide FOV. (bottom) SEM micrograph of a similar area.

Plate 59 - Pattern 4-3 - Scatterometry Lines. (top) GDS II drawing of Pattern 4-3 at a smaller FOV showing some of the detail of the fine patterns. (bottom) SEM micrograph of several of the test patterns.

Plate 60 - Pattern 4-3 - Scatterometry Lines. (top) GDS II drawing of Pattern 4-3 at a smaller FOV showing some of the detail of the fine patterns. (bottom) SEM micrograph of the L250; P1500 pattern.

Plate 61 - Pattern 4-4 - Scatterometry Trenches. (top) GDS II drawing of Pattern 4-4 showing some of the detail of the fine patterns. (bottom) SEM micrograph of a similar area of the pattern.

Plate 62 - Pattern 4-4 - Scatterometry Trenches. (top) GDS II drawing of Pattern 4-4 at a smaller FOV showing some of the detail of the fine patterns. (bottom) SEM micrograph of the L250; P 750 pattern.

\section{Section 5}

Plate 63. Section 5-0 - Overall. (top) GDS II line drawing of the entire RM 8820 pattern highlighting Section 5 with the 10 additional major sub-patterns numerically identified. (bottom) GDS II detail drawing of Section 5 showing the 10 additional major sub-patterns included in this section.

Plate 64 - Pattern 5-1 - Holes. (top) GDS II drawing of the contact holes Pattern 5-1 at wide FOV. (bottom) SEM micrograph of a similar area.

Plate 65 - Pattern 5-1 - Contact Holes. (top) GDS II drawing of the contact holes Pattern 5-1 a smaller FOV showing some of the detail of the fine patterns. (bottom) SEM micrograph of a similar area. 
Plate 66 - Pattern 5-1 - Contact Holes. (top) GDS II drawing of Pattern 5-1 at a reduced FOV showing some of the detail of the fine pattern with a nominal $250 \mathrm{~nm}$ contact holes and $880 \mathrm{~nm}$ pitch. (bottom) SEM micrograph of a similar area.

Plate 67 - Pattern 5-1 - Contact Holes. (top) SEM micrograph of contact hole pattern of the contact holes Pattern 5-1 at wide FOV. (bottom) SEM micrograph of a smaller FOV showing some of the detail of the fine details of Pattern 5-1.

Plate 68 - Pattern 5-2 - Scatterometry 2 Series Cells. GDS II drawing of the scatterometry lines Pattern 5-2 at wide FOV.

Plate 69 - Pattern 5-2 - Scatterometry 2 Series Cells. (top) GDS II drawing of the scatterometry lines Pattern 5-2 at wide FOV. (bottom) GDS II drawing of Pattern 5-2 at a reduced FOV showing some of the fine detail of a pattern with 19:1 ratio.

Plate 70 - Pattern 5-2 - Scatterometry 2 Series Cells. (top) SEM micrograph of Pattern 5-2 at wide HFW. (bottom) SEM micrograph of Pattern 5-2 at a reduced HFW showing some of the fine detail of a pattern with 19:1 ratio.

Plate 71 - Patterns 5-3, 5-4, and 5-5 - Scatterometry. GDS II drawing of the scatterometry lines Pattern $5-3,5-4,5-5$ and 5-6 at wide FOV.

Plate 72 - Pattern 5-3 - Scatterometry trenches. GDS II drawing of the scatterometry trenches Pattern 5-3 at wide FOV.

Plate 73 - Pattern 5-3 - Scatterometry trenches. (top) SEM micrograph of Pattern 5-3 at wide FOV (bottom) SEM micrograph of Pattern 5-3 at a smaller HFW showing some of the detail of the fine patterns.

Plate 74 - Pattern 5-3 - Scatterometry trenches. (top) SEM micrograph of Pattern 5-3 at wide FOV (bottom) SEM micrograph of Pattern 5-3 at a smaller HFW showing some of the detail of the fine patterns.

Plate 75 - Pattern 5-4 - Scatterometry lines. GDS II drawing of the scatterometry lines Pattern 5-3 at wide FOV.

Plate 76 - Pattern 5-4 - Scatterometry lines (top) GDS II drawing of the scatterometry lines Pattern 5-4 at wide FOV. (bottom) SEM micrograph of a similar area.

Plate 77 - Pattern 5-4 - Scatterometry lines (top) GDS II drawing of the scatterometry lines Pattern 5-4 at wide FOV. (bottom) SEM micrograph of a similar area.

Plate 78 - Pattern 5-5 - Scatterometry lines. GDS II drawing of the scatterometry lines Pattern 5-3 at wide FOV. 
Plate 79 - Pattern 5-5 - Scatterometry lines (top) GDS II drawing of the scatterometry lines Pattern 5-5 at wide FOV. (bottom) SEM micrograph of a similar area.

Plate 80 - Pattern 5-5 - Scatterometry lines (top) GDS II drawing of the scatterometry lines Pattern 5-5 at wide FOV. (bottom) SEM micrograph of a similar area.

Plate 81 - Patterns 5-6, 5 - 7 and 5 - 8 - Line Edge Roughness. (top) GDS II line drawing of the line edge roughness patterns. (bottom) SEM micrograph of a similar area.

\section{Explanation:}

Line Edge Roughness (LERBEN). The module contains 20 um long lines with various assist features for printing intentional periodic roughness artifacts similar to above. Amplitude: $10 \mathrm{~nm}$ to $100 \mathrm{~nm}$ (in $10 \mathrm{~nm}$ steps), $120 \mathrm{~nm}$, and $150 \mathrm{~nm}$. The periodicity is: 50 to 260 (10 nm steps), 280 to 340 (20 nm steps), 370, 400, 450, 500; the tabs are 1/3 of the periodicity. These additional LER patterns were designed by Benjamin Bunday of SEMATECH. The different amplitudes and periodicities and widths of the carrier lines are shown, with different styles of assist features, and each module is repeated 4 times for giving redundancy in cases of resist shrinkage.

Plate 82 - Pattern 5-6 - Line Edge Roughness. (top) GDS II line drawing of the line edge roughness patterns of Pattern 5 - 6. (bottom) SEM of a similar area of the $140 \mathrm{~nm}$ LER structure

Plate 83 - Pattern 5-6 - Line Edge Roughness. (top) GDS II line drawing of the line edge roughness patterns of Pattern 5 - 6 showing compound square wave roughness. (bottom) SEM of a similar area of the LER structure.

Plate 84 - Pattern 5-7 - Line Edge Roughness. (top) GDS II line drawing of the line edge roughness patterns of Pattern 5 - 7 showing the of the four line square wave periodicity. (bottom) SEM of a similar area of the structure.

Plate 85 - Pattern 5-7 - Line Edge Roughness. (top) GDS II line drawing of the line edge roughness patterns of Pattern 5 - 7 showing the centered assist and diagonal modules. (bottom) SEM of a similar area of the structure.

Plate 86 - Pattern 5-8 - Line Edge Roughness. (top) GDS II line drawing of the line edge roughness at reduced FOV of the four line square wave periodicity. (bottom) SEM micrograph of a similar area.

Plate 87 - Pattern 5-8 - Line Edge Roughness. (top) GDS II line drawing of the line edge roughness at reduced FOV of the four line square wave periodicity. (bottom) SEM micrograph of a similar area.

Plate 88 - Pattern 5 - 8 - Line Edge Roughness. (top) GDS II line drawing of the line edge roughness at reduced FOV of the four line square wave periodicity. (bottom) SEM micrograph of a similar area.

Plate 89 - Pattern 5 - 9 - SEM Pattern Recognition. (top) GDS II line drawing of the SEM Pattern Rec- 
ognition module at a wide FOV. (bottom) SEM micrograph of a similar area.

Explanation:

SEM Pattern Recognition. This module is an array of: 1) pattern recognition symbols of various sizes with and without dummy fill. The symbol sizes are varied and are filled with $180 \mathrm{~nm}$ line/spaces and in vertical and horizontal directions, others are solid and some are filled with $180 \mathrm{~nm}$ contact holes.

Plate 90 - Pattern 5 - 9 - SEM Pattern Recognition. (top) GDS II line drawing of the SEM Pattern Recognition module at a reduced FOV. (bottom) SEM micrograph of a similar area.

Plate 91 - Pattern 5 - 9 - SEM Pattern Recognition. (top) SEM Micrograph of the SEM Pattern Recognition module at an HFW of (top) $159 \mu \mathrm{m}$ and (bottom) $84.7 \mu \mathrm{m}$.

Plate 92 - Pattern 5-10 - Line End. (top) GDS II line drawing of the line end pullback and line end pull back links patterns a wide FOV. (bottom) SEM micrograph of a similar area.

Explanation:

LineEnd. These modules are designed to show effects of line end shortening and enable reliable measurement of this unavoidable consequence of sub-wavelength lithography. There are two types of line end shortening structures: 1) horizontal/vertical block pattern, 2) horizontal link pattern. OPC is added to the last cell in both types as indicated; $15 \mathrm{~nm}$ is added to each side on the block pattern and $10 \mathrm{~nm}$ is added to each side of the link pattern. Block size/space: $150 \mathrm{~nm}$ width $150 \mathrm{~nm}$ space, $160 \mathrm{~nm}$ width $150 \mathrm{~nm}$ space, $150 \mathrm{~nm}$ width $200 \mathrm{~nm}$ space, $160 \mathrm{~nm}$ width $200 \mathrm{~nm}$ space $150 \mathrm{~nm}$ width $200 \mathrm{~nm}$ space with Optical proximity correction (OPC). These are in gratings large enough to be used with Optical Critical Dimension (OCD).

LineEnd shortening. There are two types of line end shortening structures: 1) horizontal/vertical block pattern and 2) horizontal link pattern. OPC is added to the last cell in both types as indicated; $15 \mathrm{~nm}$ is added to each side on the block pattern and $10 \mathrm{~nm}$ is added to each side of the link pattern. Block size/space: $150 \mathrm{~nm}$ width $150 \mathrm{~nm}$ space, $160 \mathrm{~nm}$ width $150 \mathrm{~nm}$ space, $150 \mathrm{~nm}$ linewidth $200 \mathrm{~nm}$ space, $160 \mathrm{~nm}$ width $200 \mathrm{~nm}$ space $150 \mathrm{~nm}$ width $200 \mathrm{~nm}$ space with OPC.

Plate 93 - Pattern 5-10 - Line End Pullback. (top) GDS II line drawing of the line end pullback and line end pull back links patterns a wide FOV. (bottom) SEM micrograph of a similar area.

Plate 94 - Pattern 5 - 10 - Line End Pullback. SEM Micrographs of the $150 \mathrm{~nm}$ (top) and $160 \mathrm{~nm}$ (bottom) lines. 


\section{Section 6}

Plate 95 - Section 6-0 Overall. (top) GDS II line drawing of the entire RM 8820 pattern highlighting Section 6 with the 9 additional major sub-patterns numerically identified. (bottom) GDS II detail drawing of section 6 showing the 9 additional major sub-patterns included in this section. Pattern 6-1 is also seen in the center of this module.

Plate 96 - Patterns 6-2, 6-3 and 6-4. Wide FOV of GDS II line drawing of Patterns 6-2 (SEM overlay), 6-3 (scatterometry) and 6-4 (array target) at a wide FOV. (bottom) SEM micrograph of a similar area.

Plate 97 - Pattern 6-2 - SEM Overlay. GDS II drawing of Pattern 6-2 at a smaller FOV showing some of the detail of the fine patterns. There are several line groups with different design rules and different built-in offsets of the center line for testing SEM overlay measurement capabilities.

Explanation:

There are several line groups with different design rules and different built-in offsets of the center line for testing SEM overlay measurement capabilities.

SEM Overlay. The SEM overlay structures were designed with multiple groups of three lines. The groups are placed both horizontally and vertically in the module. CDs are $100 \mathrm{~nm}, 140 \mathrm{~nm}, 200 \mathrm{~nm}$, $250 \mathrm{~nm}, 300 \mathrm{~nm}$. The inner line is offset by the following steps in the $X$ and $Y$ direction:-150 nm, -100 $\mathrm{nm},-50 \mathrm{~nm},-20 \mathrm{~nm}, 0 \mathrm{~nm}, 20 \mathrm{~nm}, 50 \mathrm{~nm}, 100 \mathrm{~nm}, 150 \mathrm{~nm}$. All polarities were used for the SEM Overlay structures.

Plate 98 - Pattern 6-3 - Array Target. (top) GDS II line drawing of the array target module at a wide FOV. (bottom) SEM micrograph of a similar area.

\section{Explanation:}

Array Target. The array target is composed of two types of modules. Module 1 has four lines with three spaces and the Module 2 has 10 lines and 9 spaces. The critical dimensions (CDs) are $100 \mathrm{~nm}, 150$ $\mathrm{nm}, 250 \mathrm{~nm}, 300 \mathrm{~nm}$ and $350 \mathrm{~nm}$. The pitch starts at 1:1 and then is spaced as follows: $100 \mathrm{~nm}, 150$ $\mathrm{nm}, 200 \mathrm{~nm}, 250 \mathrm{~nm}, 300 \mathrm{~nm}, 350 \mathrm{~nm}, 400 \mathrm{~nm}, 450 \mathrm{~nm}, 500 \mathrm{~nm}, 1000 \mathrm{~nm}, 2000 \mathrm{~nm}$. Therefore, the $200 \mathrm{~nm}$ line would start as a 1:1 and increase in spacing following the above spacing requirements.

Plate 99 - Pattern 6-3 - Array Target. (top) GDS II line drawing of the array target module at a wide FOV. (bottom) SEM micrograph of a similar area.

Plate 100 - Pattern 6-3 - Array Target. SEM micrograph of a segment of the Array Target pattern.

Plate 101 - Pattern 6-4 - Scatterometry 140 Module. (top) GDS II line drawing of the scatterometry 140 module module at a wide FOV. (bottom left and right) GDS II drawing of a reduced FOV of some 
of the fine detail of the patterns.

Plate 102 - Pattern 6-4 - Scatterometry 140 Module. (top) SEM micrograph of the 100 x 100 array at low HFW (bottom) SEM micrograph of higher HFW showing the individual lines.

Plate 103 - Pattern 6-5 - NIST proposed AFM step height pattern (top) GDS II line drawing of a portion of ta proposed NIST pattern for a step height standard module at a wide FOV. (bottom) SEM micrograph of the NIST pattern module showing the fine structure in the center.

\section{Explanation:}

NIST (proposed) Step Height Prototype Pattern. This pattern is designed to accommodate issues specific to AFMs. The large direction pattern surrounding the critical dimension target is to make locating the target easier for AFMs with limited navigation and pattern recognition capability. The large target and pitch size is for assessing large field of view scanning. (Designed by Ronald Dixson of NIST).

Plate 104 - Pattern 6-5 - NIST (proposed) AFM Step Height Pattern. (top) GDS II line drawing of a portion of the NIST prototype pattern for step height standard module at a wide FOV. (bottom) SEM micrograph of the NIST pattern module showing the fine structure in the center.

Plate 105. Pattern 6-5 NIST (proposed) AFM Step Height Pattern. SEM micrograph of the center of the NIST pattern module showing the fine structure.

Plate 106 - Pattern 6-6 - High Resolution Overlay. (top) GDS II line drawing of the high resolution overlay targets module Pattern 6-8 and 6-9 at a wide FOV. (bottom) SEM micrograph of a similar area.

Explanation:

High Resolution Overlay Targets. The High Resolution Module is made of several $20 \times 20$ micrometer boxes of line/trench modules. CDs are $100 \mathrm{~nm}, 150 \mathrm{~nm}, 200 \mathrm{~nm}, 250 \mathrm{~nm}, 300 \mathrm{~nm}, 400 \mathrm{~nm}, 500 \mathrm{~nm}$. The space widths are $500 \mathrm{~nm}, 1000 \mathrm{~nm}, 2000 \mathrm{~nm}, 3000 \mathrm{~nm}, 4000 \mathrm{~nm}, 5000 \mathrm{~nm}$. There are three cells for each CD/space: the first cell has a $30 \%$ offset in the positive X axis, the second cell has a $30 \%$ offset in the positive Y axis, and the third cell has no offset but is 180 degrees out of phase. Because this pattern was originally intended for an overlay reticle set, only a single level target is displayed.

Plate 107 - Pattern 6-6 - High Resolution Overlay. (top) GDS II line drawing of the high resolution overlay targets module Pattern 6-6 at a wide FOV. (bottom) SEM micrograph of a similar area.

Plate 108 - Pattern 6-6 - High Resolution Overlay. (top) GDS II line drawing of the high resolution overlay targets module Pattern 6-6 at a wide FOV. (bottom) SEM micrograph of a similar area.

Plate 109 - Pattern 6-6 - High Resolution Overlay. (top and bottom) SEM micrographs of the overlay patterns at higher HFW. 
Plate 110 - Pattern 6-6 - High Resolution Overlay. (top) GDS II line drawing of the high resolution overlay targets module Pattern 6-6 at a wide FOV. (bottom) SEM micrograph of a similar area.

Plate 111 - Pattern 6-7 - Scatterometry Field Size. (top) Reduced field GDS II line drawing of several of the scatterometry targets found on Pattern 6-7 at a reduced FOV. (bottom) SEM micrograph of a similar area.

Plate 112 - Pattern 6-7 - Scatterometry Field Size. (top) Reduced field GDS II line drawing of several of the scatterometry targets found on Pattern 6-7 at a reduced FOV. (bottom) SEM micrograph of a similar area.

\section{Section 7}

Plate 113 - Section 7-0 Overall. (top) GDS II line drawing of the entire RM 2089 pattern highlighting Section 7 with the 6 additional major sub-patterns numerically identified. (bottom) GDS II detail drawing of section 7 showing the 6 additional major sub-patterns included in this section. Pattern 7-1 is also seen in the center of this module.

Plate 114 - Patterns 7-1 and 7-2. Wide FOV of GDS II line drawing of Patterns 7-1 (Fill module) and 7-2 (Cleave lines) at wide FOV.

Plate 115 - Patterns 7-1 - Fill module. (top) Wide FOV of GDS II line drawing of patterns 7-1 fill module. (below). SEM micrograph of a similar area.

\section{Explanation:}

Fill Module. Each module contains 13 sub modules as shown in Pattern 7-1. In each sub module there is dummy fill surrounding 9 targets. There are four different types of targets but in each module only one type is used. The first module contains line in dense line targets, the second module contains box in box targets, the third module contains $0.5 \mu \mathrm{m}$ trench in trench targets, and the fourth module contains $1 \mu \mathrm{m}$ frame in frame targets. The targets remain the same in each module while the dummy fill expands away from each overlay target. The fill remains a constant. $100 \mu \mathrm{m}$ away for the outer centered targets, the fill then varies from sub module to sub module. Sub modules go from left to right, top to bottom. One thing to note is that because this cell was originally intended for an overlay reticle set, you will only see a single level target.

Plate 116 - Patterns 7-1 - Fill module. (top) Low HFW SEM micrograph of the fill module. (bottom) Higher HFW micrograph of a one of the cells.

Plate 117 - Patterns 7-1 - Fill module. (top) Low HFW SEM micrograph of the fill module. (bottom) Higher HFW micrograph of a one of the cells. 
Plate 118 - Pattern 7-2 - Cleave Lines. (top) Reduced FOV GDS II line drawings of several of the horizontal cleave line targets found on Pattern 7-2. (bottom) SEM micrograph of a similar area.

Plate 119 - Pattern 7-2 - Cleave Lines. (top) Higher FOV GDS II line drawing of several of the horizontal cleave line targets found on Pattern 7-2. (bottom) SEM micrograph of a similar area.

Plate 120 - Pattern 7-3 - Multisize Scatterometry. (top) Wide FOV GDS II line drawing of several of the horizontal multisize scatterometry line targets found on Pattern 7-3. (bottom) SEM micrograph of a similar area.

Plate 121 - Pattern 7-3 - Scatterometry Defect. (top) Wide FOV GDS II line drawing of Pattern 7-3. (bottom) SEM micrograph of a similar area.

Plate 122 - Pattern 7-3 - Scatterometry Defect. (top) Low HFW SEM micrograph.of pattern 7-3. (bottom) Higher HFW SEM micrograph of a similar area.

Plate 123 - Pattern 7-4 - Scatterometry Defect. (top) Wide FOV GDS II line drawing of Pattern 7-4. (bottom) SEM micrograph of a similar area.

Plate 124 - Pattern 7-4 - Scatterometry Defect. (top) Low HFW SEM micrograph of Pattern 7-4. (bottom) Higher HFW SEM micrograph of the finer detail of the patterns.

Plate 125 - Pattern 7-5 - Phase Imbalance. (top) Wide FOV GDS II line drawing of the phase imbalance pattern. (bottom) SEM micrograph of a similar area.

Plate 126 - Pattern 7-5 - Phase Imbalance. (top) Wide FOV GDS II line drawing of the phase imbalance pattern. (bottom) SEM micrographs of a similar area.

Plate 127 - Pattern 7-5 - Phase Imbalance. (top) Higher HFW SEM micrographs of Pattern 7-5. (bottom) Higher HFW of one of the phase imbalance patterns.

Plate 128 - Pattern 7-6 - Multiple Scatter Lines. (top) Wide FOV GDS II line drawing of several of the multiple scatter lines targets found on Pattern 7-6. (bottom) SEM micrograph of a similar area.

Plate 129 - Pattern 7-6 - Multiple Scatter Lines. (top) Wide FOV GDS II line drawing of L100P295 pattern found on Pattern 7-6. (bottom) SEM micrograph of a similar area.

Plate 130 - Pattern 7-6 - Multiple Scatter Lines. SEM micrograph at a smaller HFW of the lines found on Pattern 7-6. 
SECTION 1 
Plate 2. Section 1-0 - Overall. (top) GDS II line drawing of the entire RM 8820 pattern highlighting Section 1 with the 8 additional major sub-patterns numerically identified. (bottom) GDS II detail drawing of Section 1 showing the 8 additional major sub-patterns included in this section. 


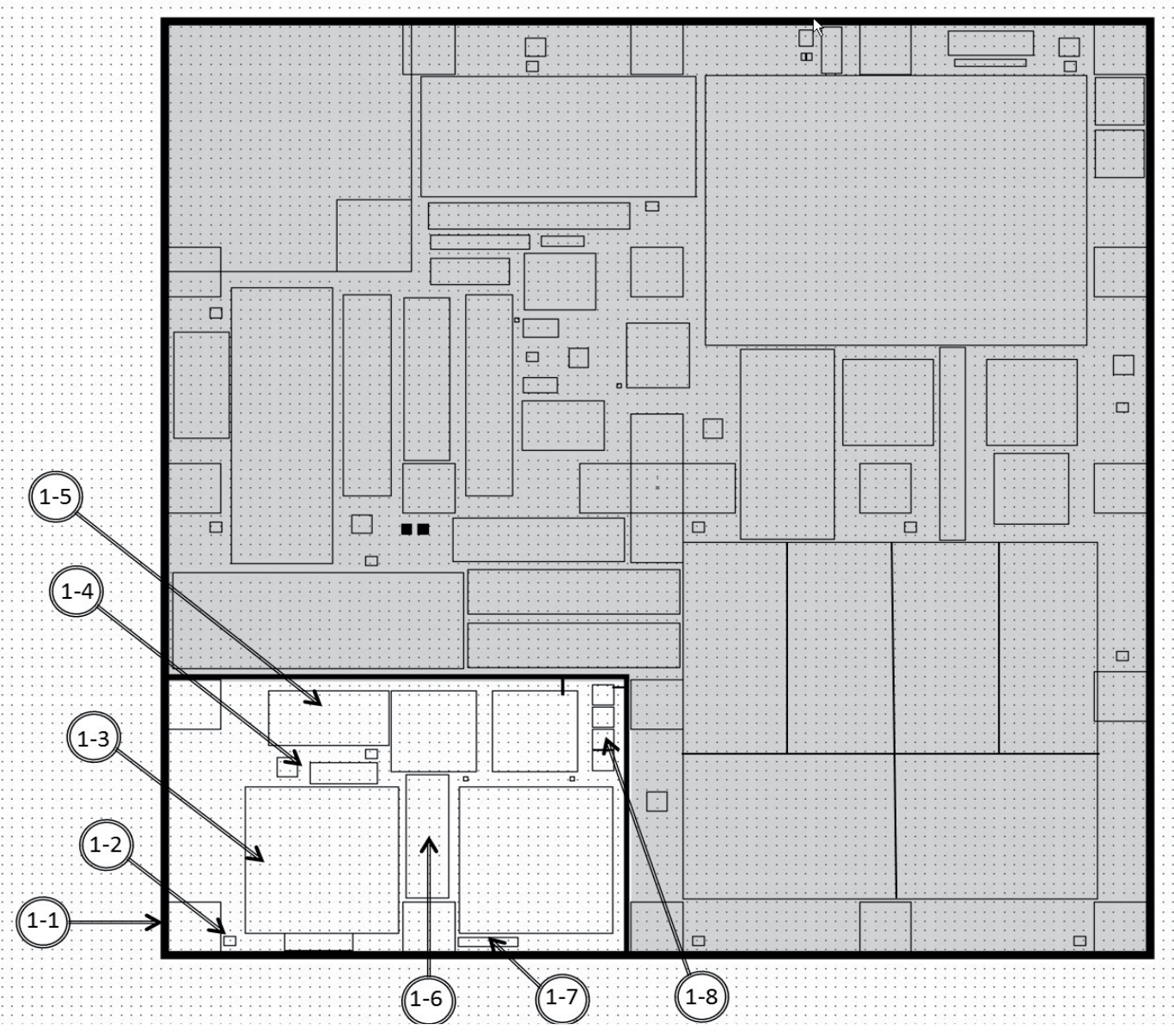

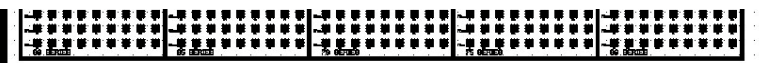

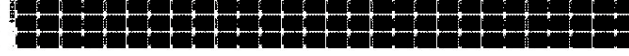

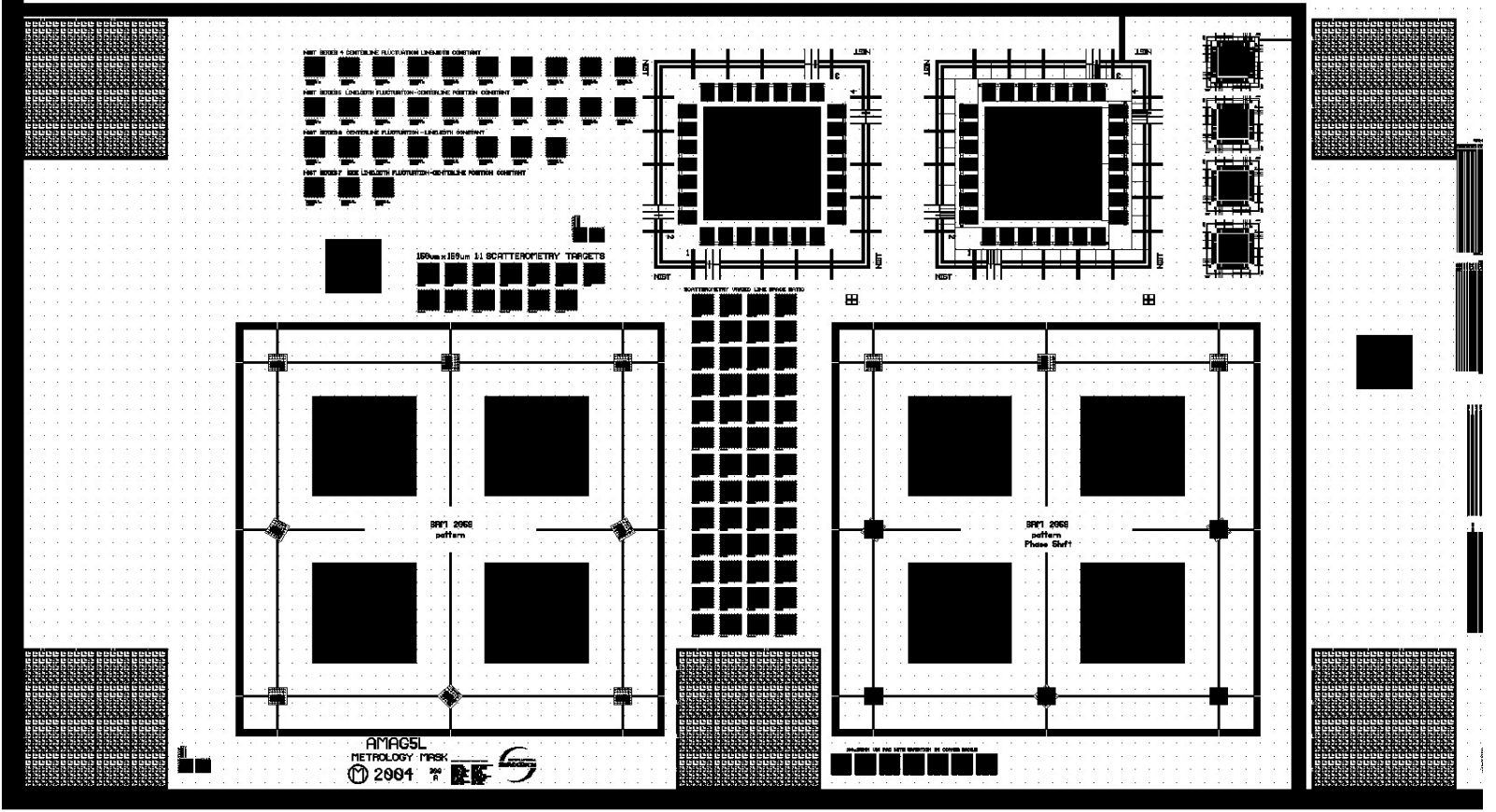

Plate 2 - RM 8820 Section 1 
Plate 3 - Pattern 1-1 - Critical Dimension Measurement (CD). (top) GDS II drawing of critical dimension Pattern 1-1 at low magnification. The smaller Pattern 1-2 is also represented in this view. (bottom) SEM micrograph of Pattern 1-1 and 1-2 in the same field. Pattern 1-1 is replicated 25 times across the chip. (But, these patterns are only once at their first occurrence).

\section{Explanation:}

CD measurement features. Dimensional Control Patterns (DCP) are useful for critical dimension measurement testing in metrology instruments. Each built-in pattern recognition structure includes: Lines: 1:1, 2:1 \& 3:1; Isolated (Iso) space; Contact Holes 1:1, 2:1, \& 4:1. Contact Holes only exist in the $80 \mathrm{~nm}$ cells and above due to tool limitations. This feature was designed with several important factors in mind: a) to maximize robustness of the smallest of the features for ensuring that testing would be valid to the smallest possible nodes; $b$ ) to ensure a wide variety of feature sizes for linearity testing; c) for extremely simple, intuitive navigation; d) availability of features in both horizontal and vertical directions; e) many repetitions across the scanner field, allowing across-field variation to be measured if necessary; and f) many redundant, identical copies of the same features to allow for repetition of different experiments that involve sample damage by SEM, such as with contamination or photoresist shrinkage (the key concept could be described as many "disposable features" within each die).

Pattern 1.1 represents the SEMATECH version of what many in the industry describe as "L-bars". The $5 \times 5$ array shown on the left is exactly $250 \mu \mathrm{m}$ square, and a $4 \times 4$ block of these always were placed together to make $1 \mathrm{~mm}$ square modules at 25 different locations across the field; thus there are 400 copies of each of the features available on each die. On the left is a close-up of a $50 \mu \mathrm{m}$ square cell within the $5 \times 5$ matrix; note that each is carefully marked with nominal size on reticle, and includes vertical and horizontal lines, contact holes, isolated trenches and a few different line/space pitches from dense to isolated, and that robust pattern recognition and feature anchors are also available. 

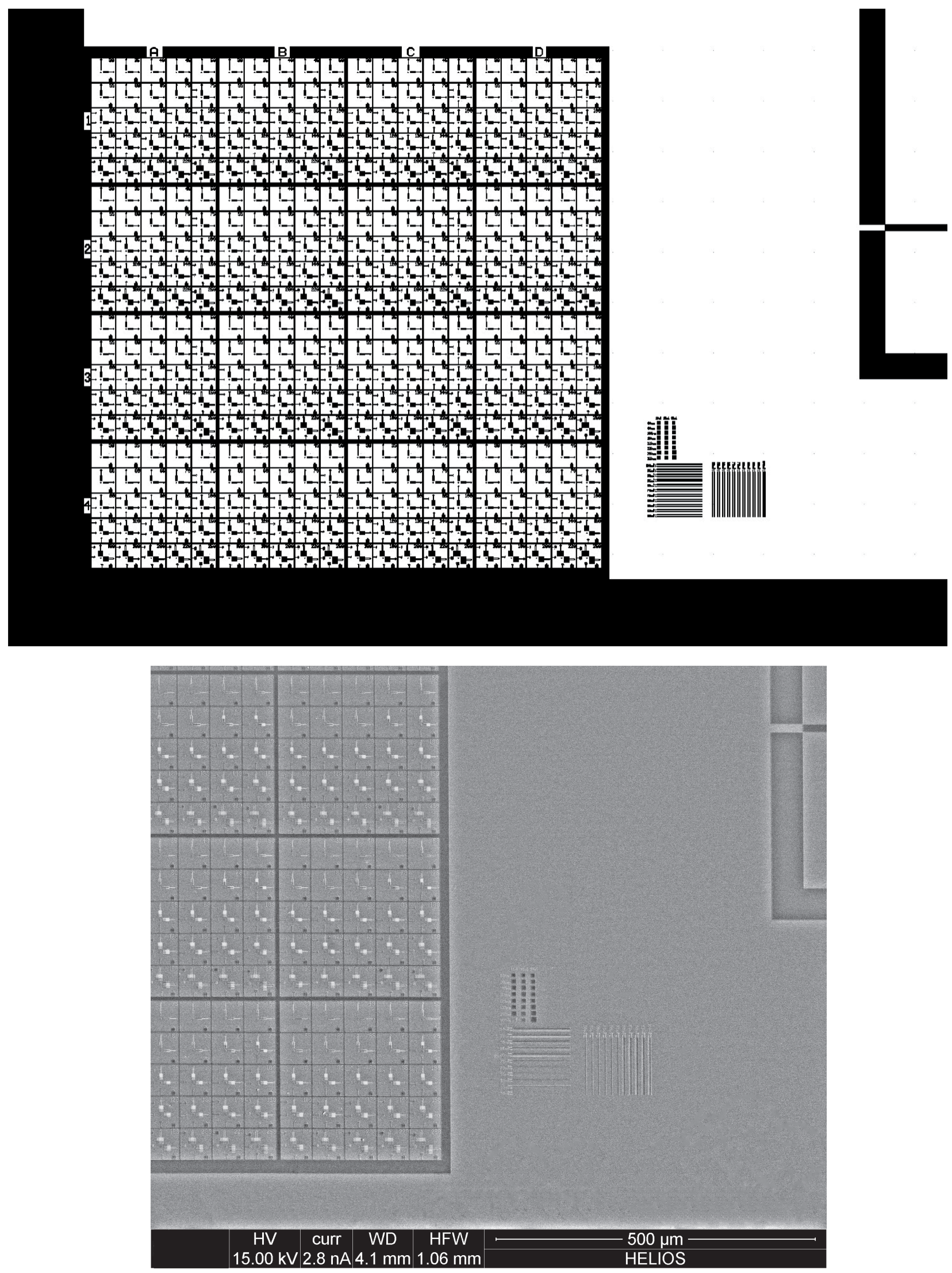

Plate 3 - Patterns 1-1 and 1-2 - Critical Dimension Measurement (CD) 
Plate 4 - Pattern 1-1 - Critical Dimension Measurement (CD). (top) GDS II drawing of critical dimension Pattern 1-1 at a higher magnification. (bottom) SEM micrograph of a similar area. 

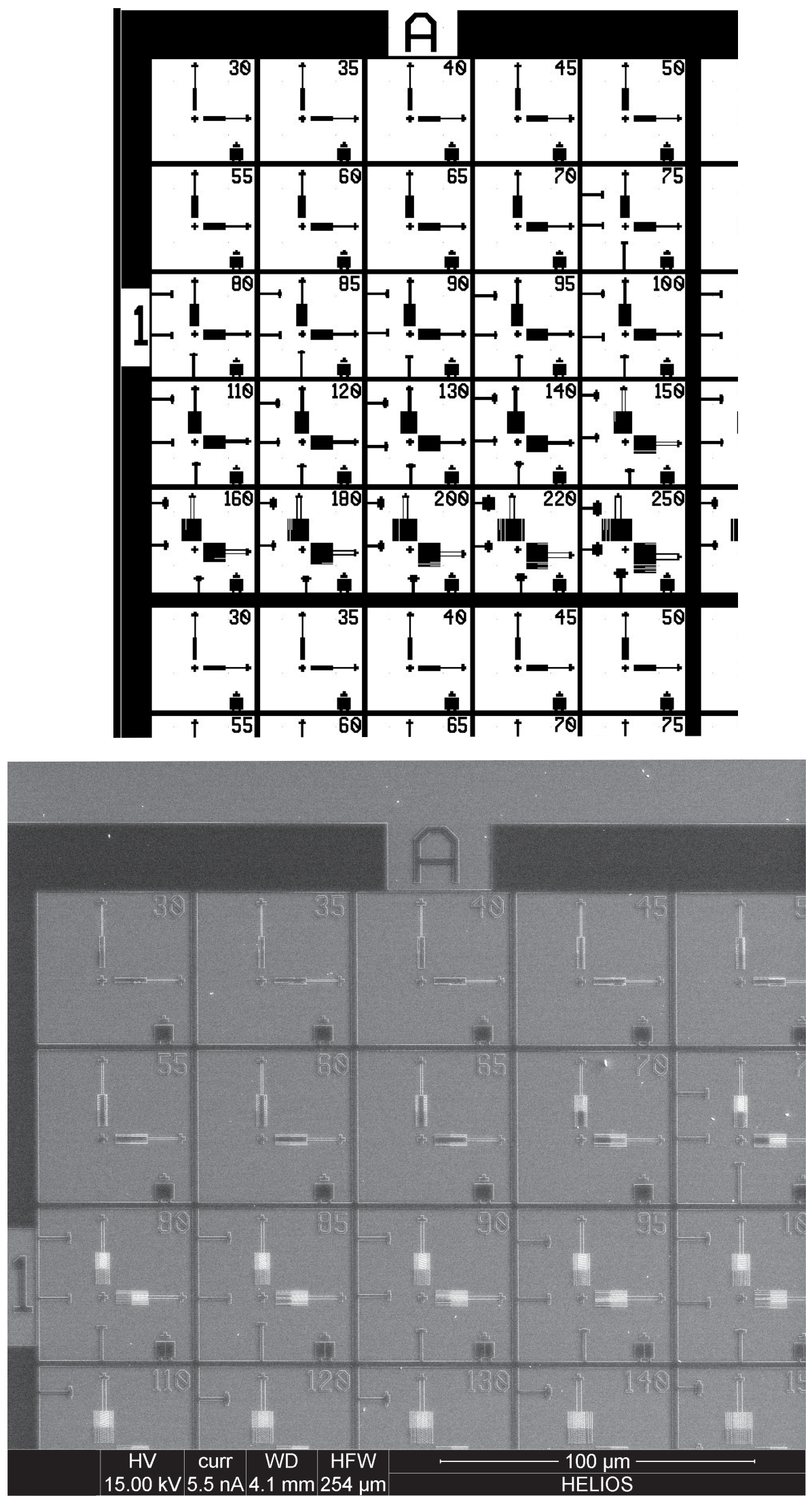

Plate 4 - Pattern 1-1 - Critical Dimension Measurement (CD) 
Plate 5 - Pattern 1-1 - Critical Dimension Measurement (CD). (top) GDS II drawing of critical dimension Pattern 1-1 at a higher magnification. (bottom) SEM micrograph of a similar area. 

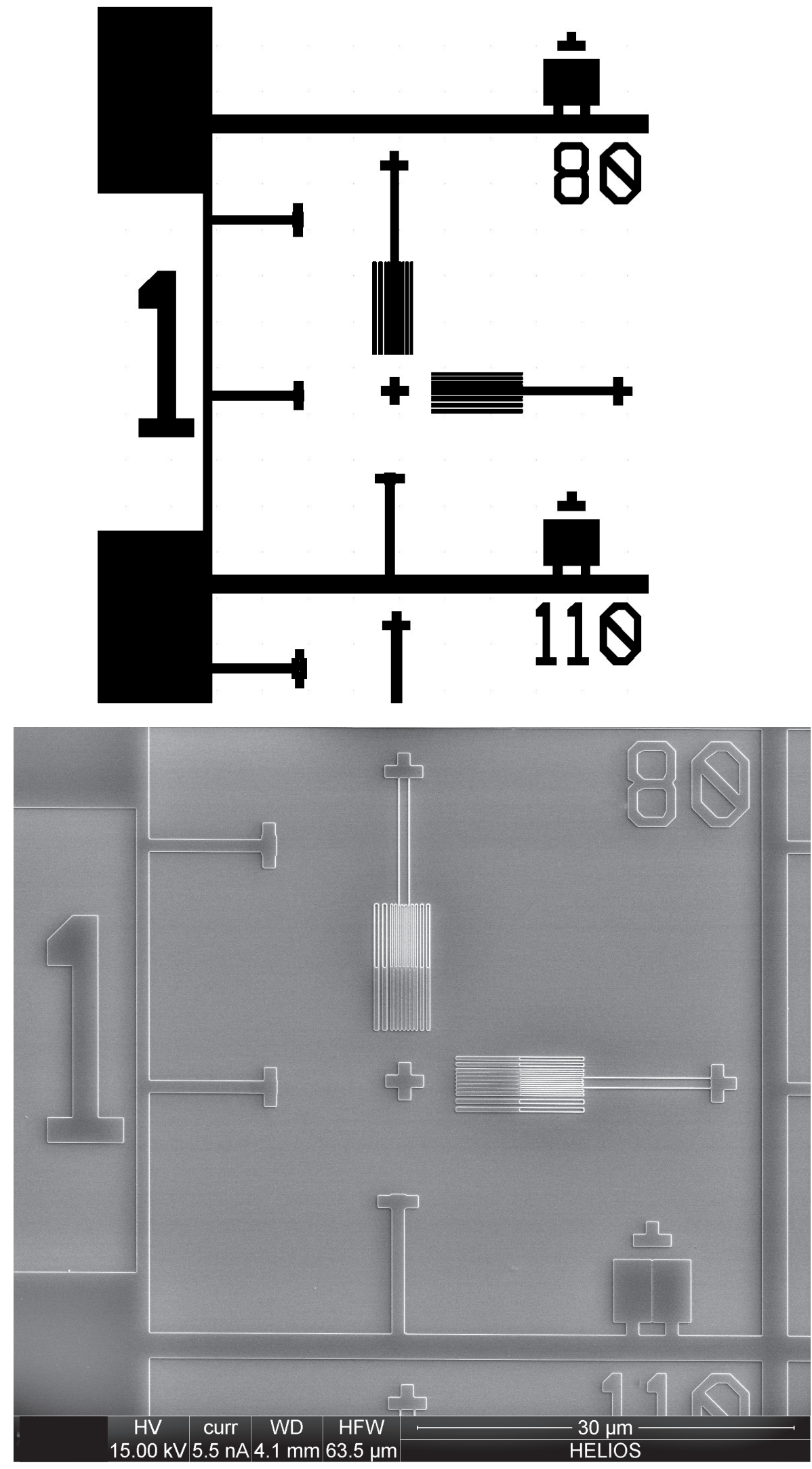

Plate 5 - Pattern 1-1 - Critical Dimension Measurement (CD) 
Plate 6 - Pattern 1-2 - Critical Dimension Measurement (CD). (top) GDS II drawing of critical dimension Pattern 1-2. (bottom) SEM micrograph of a similar area. 


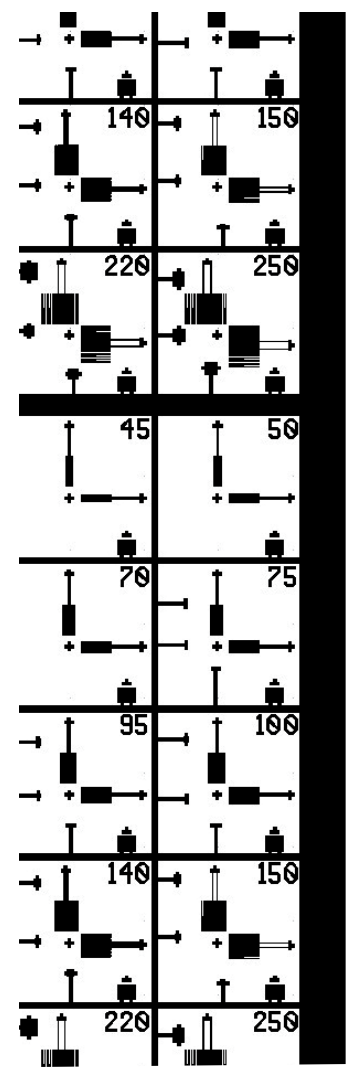

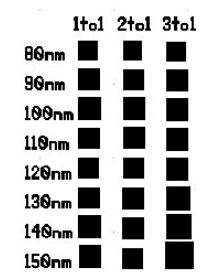

$100 \mathrm{~nm}^{\circ}$

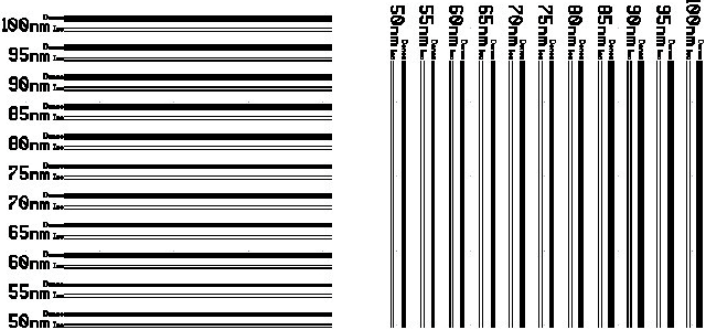

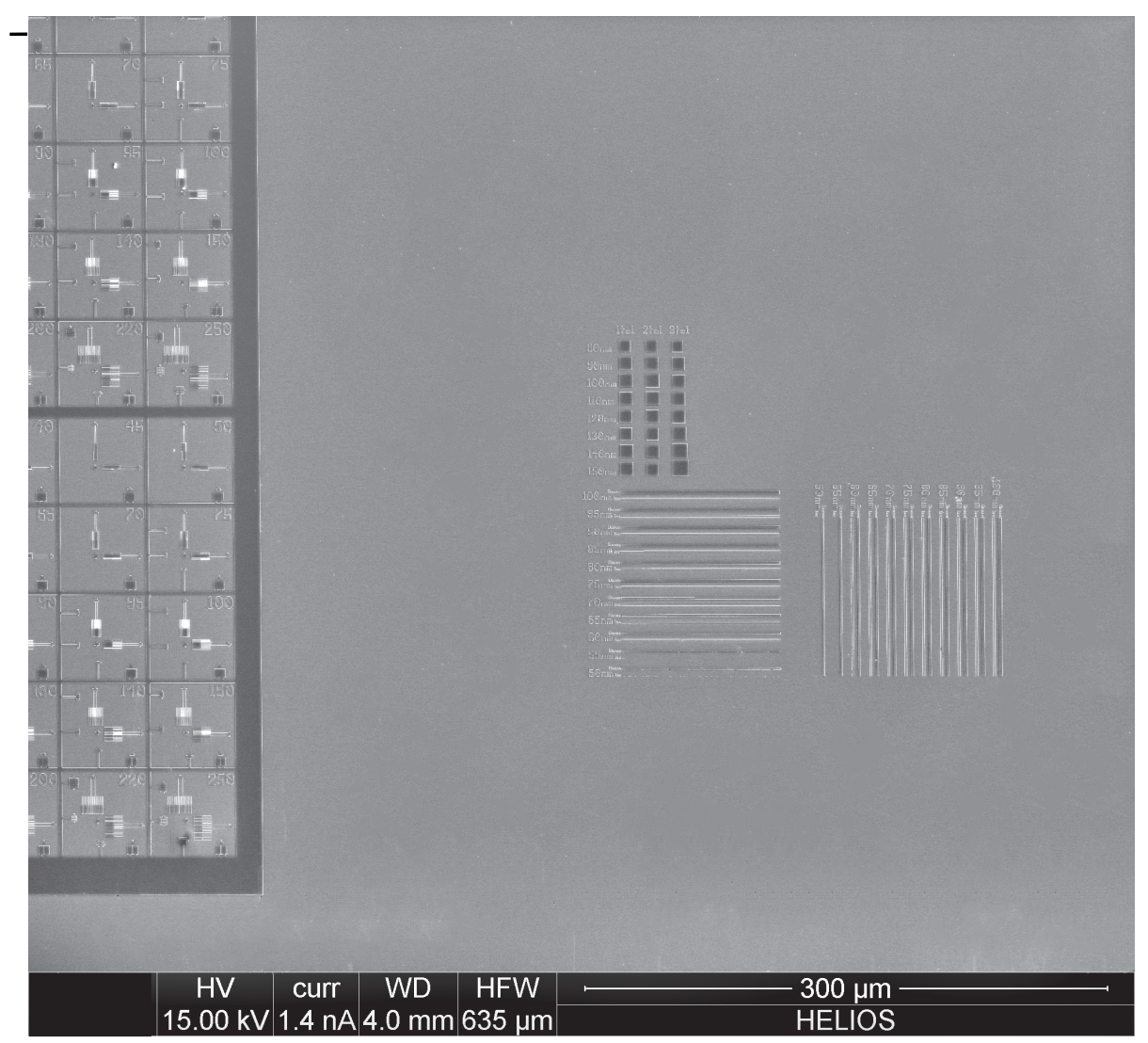

Plate 6 - Pattern 1-2 - Critical Dimension Measurement (CD) 
Plate 7 - Pattern 1-2 - Critical Dimension Measurement (CD).(top) GDS II drawing of critical dimension Pattern 1-2 at a higher magnification. (bottom) SEM micrograph of a similar area. 


\section{$130 \mathrm{~nm}$
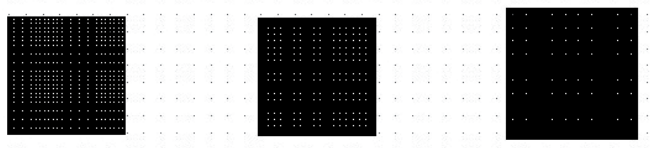 \\ $140 \mathrm{~nm}$
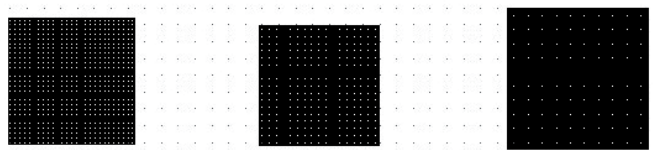 \\ $150 \mathrm{~nm}$
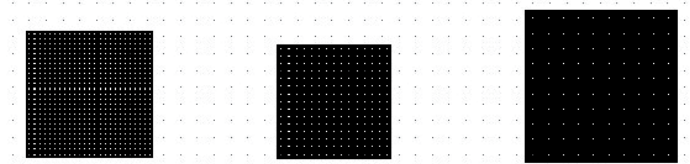

$100 \mathrm{~nm}$ m lso

$95 n \mathrm{~m} m$ lso

$98 \mathrm{~nm}$ 18on

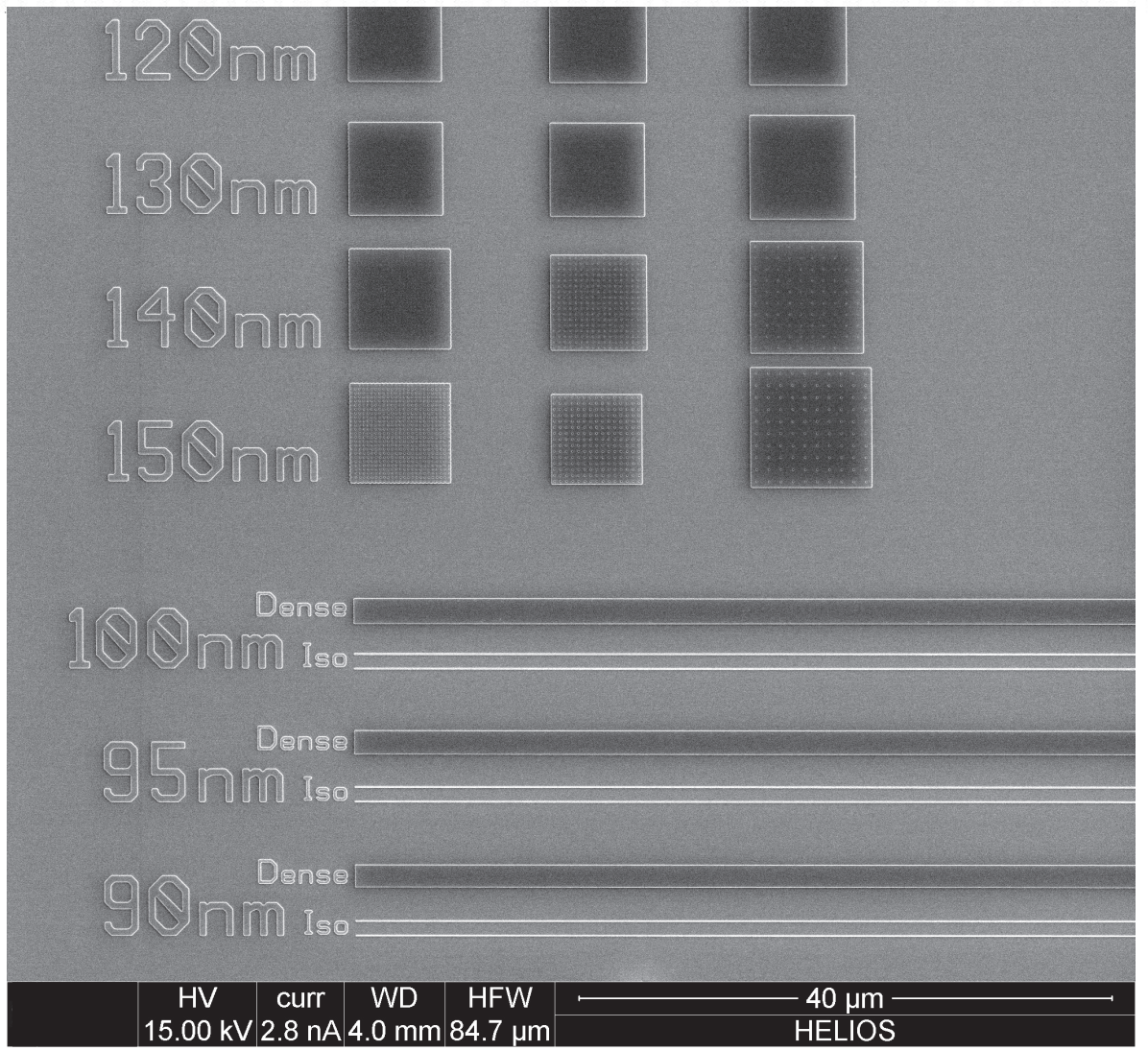

Plate 7 - Pattern 1-2 - Critical Dimension Measurement (CD). 
Plate 8 - Pattern 1-3 - SRM 2059 pattern. (top) GDS II drawing of Pattern 1-3 at low field of view. (bottom) SEM of a corner of the pattern.

\section{Explanation:}

NIST Pattern for SRM 2059. This pattern is a printing of the NIST SRM 2059 photomask optical microscope linewidth calibration standard. This is not meant to be a replacement to this standard. This is an area that combines various line and space scatterometry cells located at the center with the metrology structures of the existing SRM 2059 photomask linewidth standard (NIST SRM 2059). This area was originally designed by James Potzick of NIST. [see the NIST website for further description] 

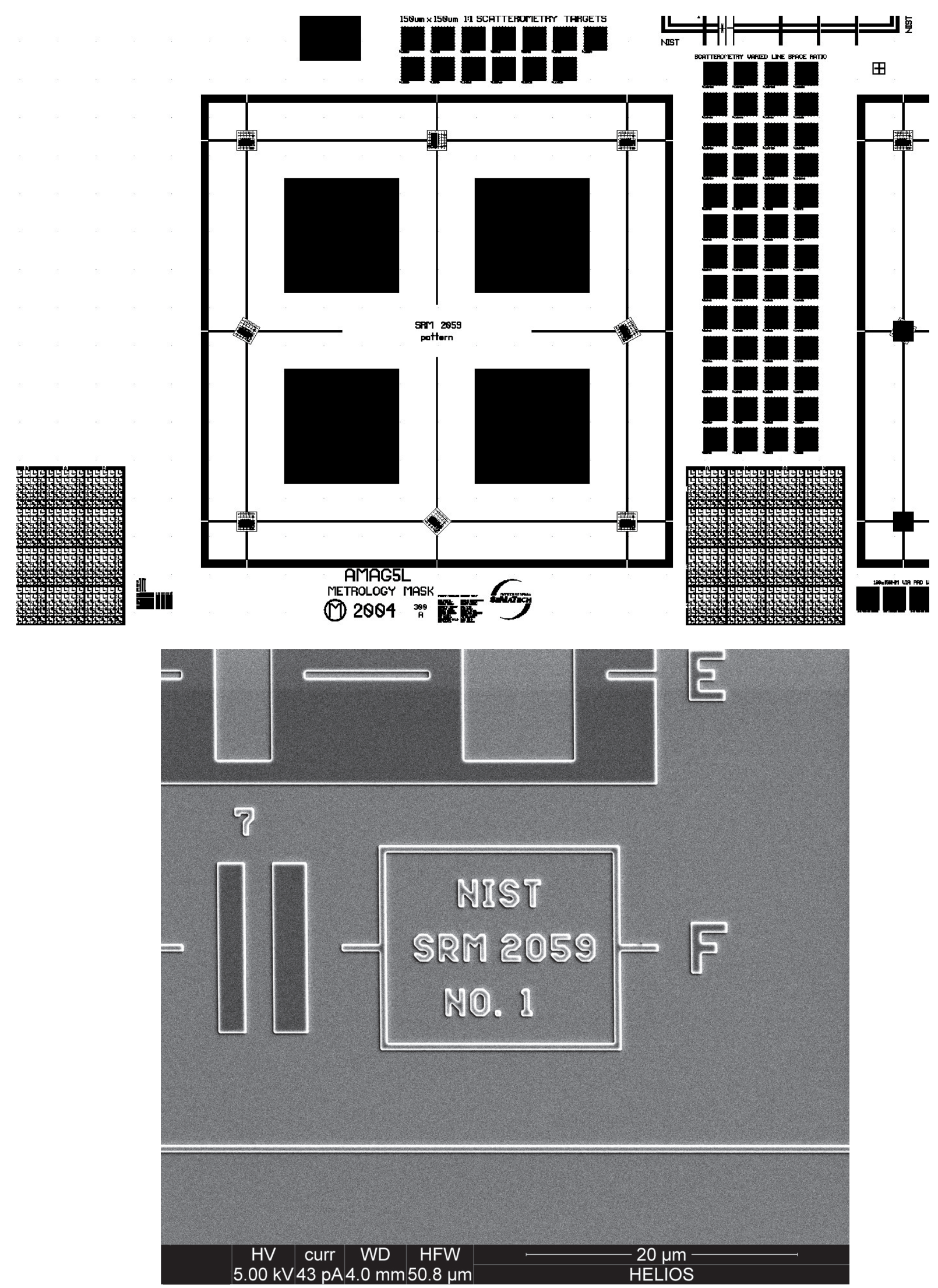

Plate 8 - Pattern 1-3 - SRM 2059 pattern 
Plate 9 - Pattern 1-3 - SRM 2059 pattern. (top) GDS II drawing of Pattern 1-3 at low field of view. (Bottom) SEM of a similar field of view. 

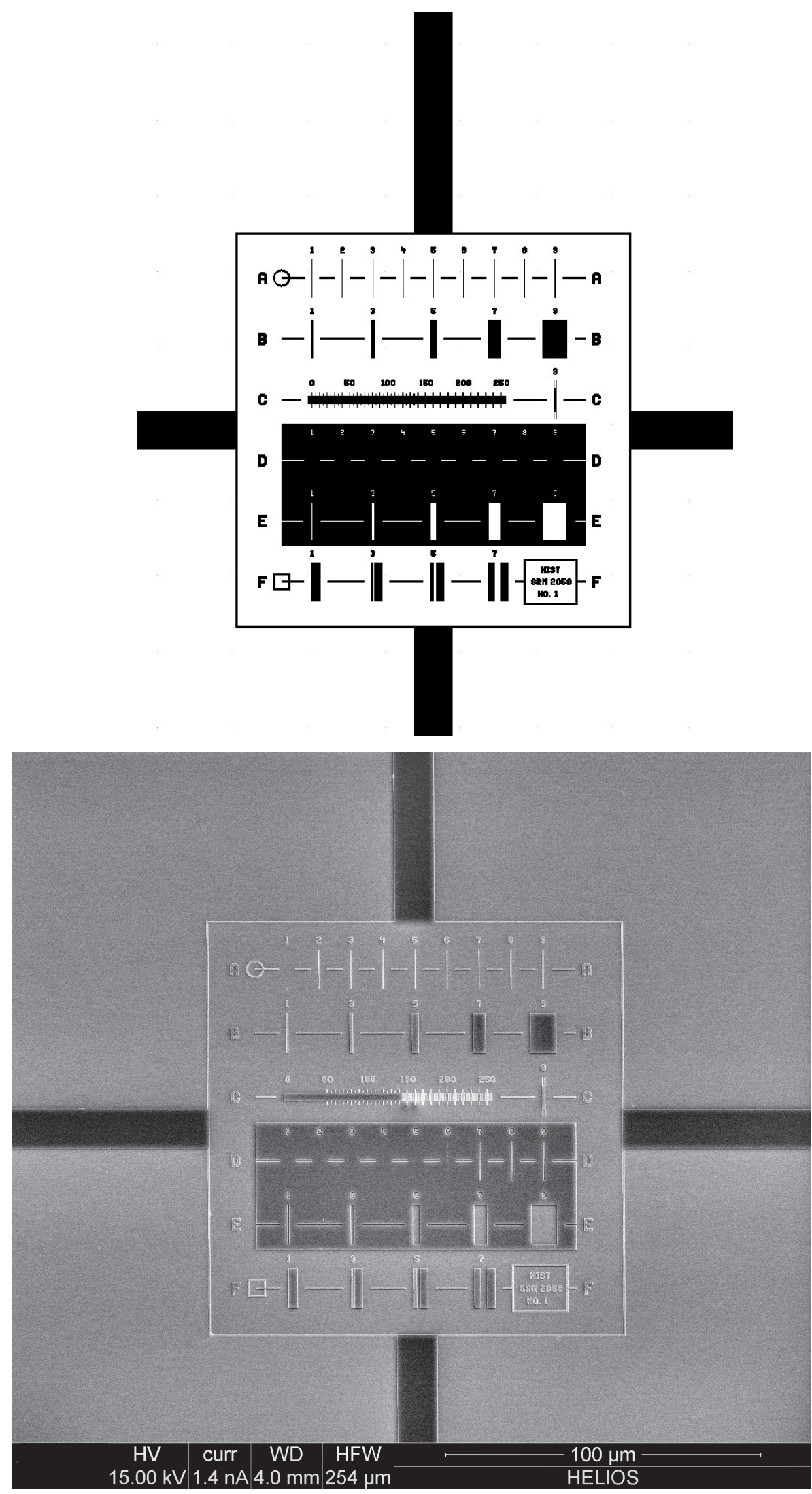

Plate 9- Pattern 1-3 - SRM 2059 pattern 
Plate 10 - Pattern 1-3 - SRM 2059 pattern. (top) GDS II drawing of one of the smaller patterns of 1-3. (bottom) SEM of a reduced field of view of some of the calibration features of SRM 2059 showing multiple pitch lines. 


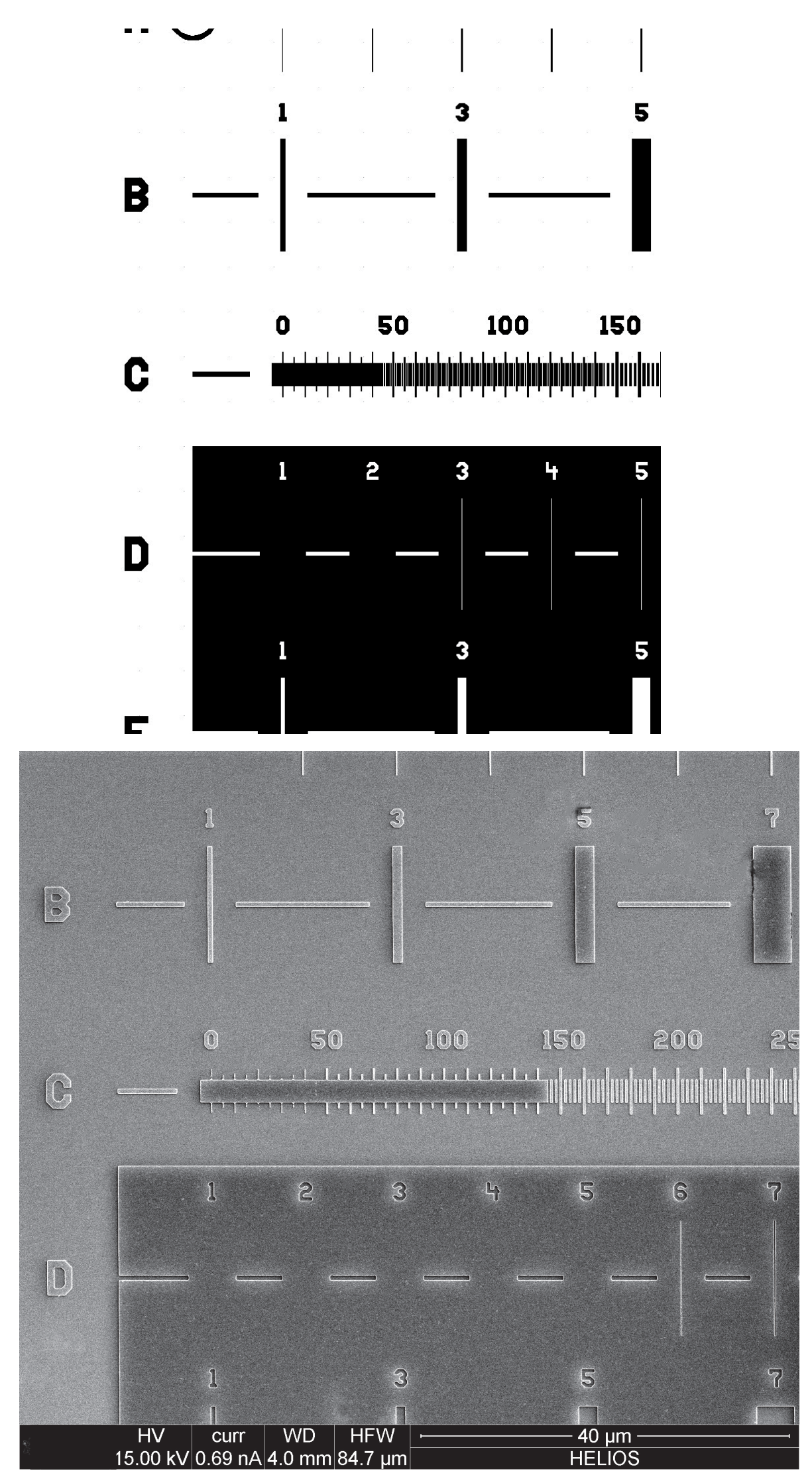

Plate 10 - Pattern 1-3 - SRM 2059 pattern 
Plate 11 - Pattern 1-4 - Scatterometry Target. (top) GDS II drawing of the $150 \mu \mathrm{m}$ by $150 \mu \mathrm{m}$ scatterometry targets. (bottom) SEM of a similar area of the RM8820 reference material.

\section{Explanation:}

Scatterometry Target. The scatterometry target uses the $150 \mathrm{~nm} \mathrm{1:1} \mathrm{trenches} \mathrm{over} \mathrm{different} \mathrm{areas.}$ The following is a list of the different areas covered by the $140 \mathrm{~nm}$ trench: $100 \times 100 \mu \mathrm{m}, 90 \times 90 \mu \mathrm{m}$, $80 \times 80 \mu \mathrm{m}, 70 \times 70 \mu \mathrm{m}, 60 \times 60 \mu \mathrm{m}, 50 \times 50 \mu \mathrm{m}, 40 \times 40 \mu \mathrm{m}, 30 \times 30 \mu \mathrm{m}, 20 \times 20 \mu \mathrm{m}$, and 10x10 $\mu \mathrm{m}$. 


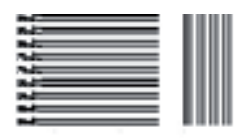

\section{8um $\times$ 158um 1:1 SCATTEROMETRY TARGE'}

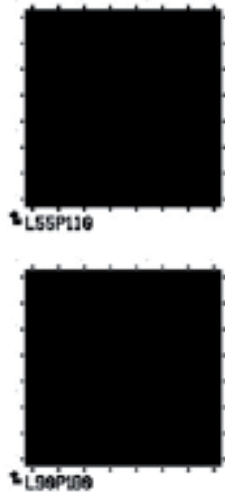

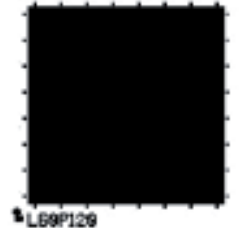

บLe9120

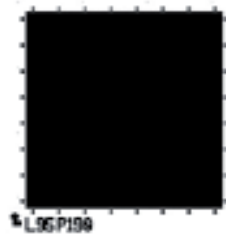

tLisapios

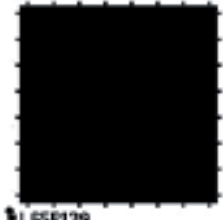

-

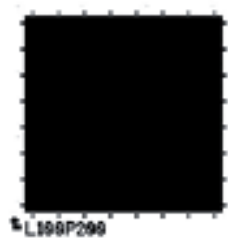

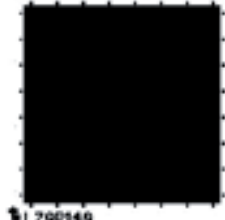

เา

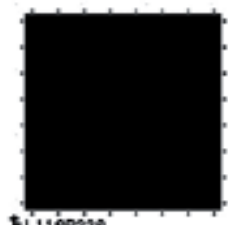

tLi1epra

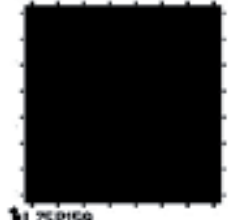

thrspiss

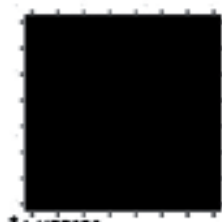

tisispose

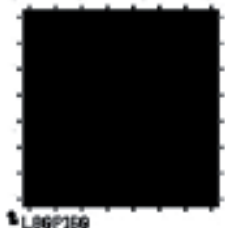

LัLeตรe

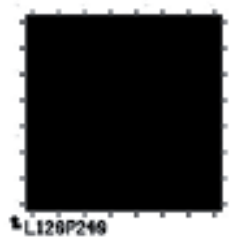

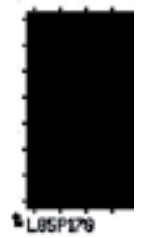
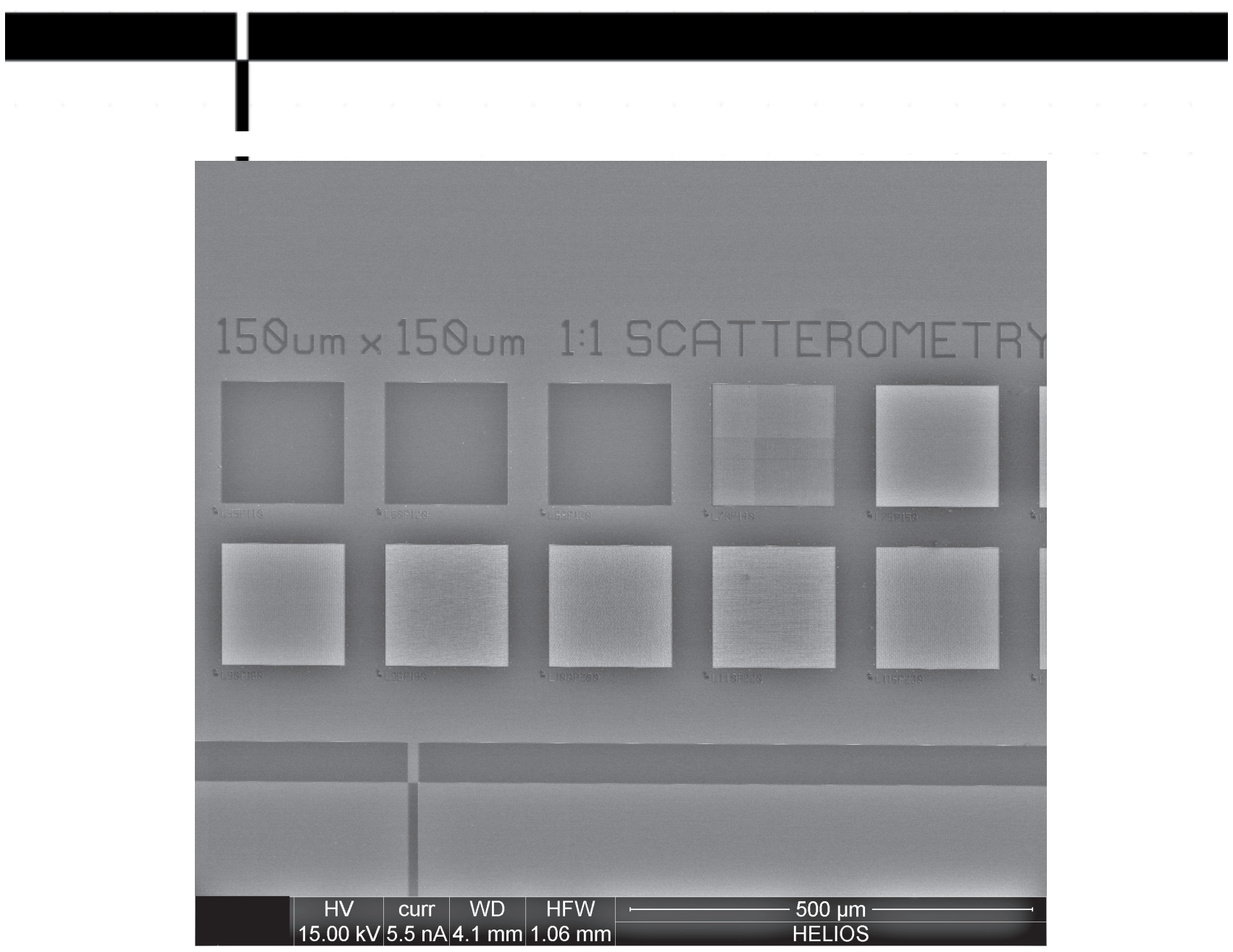

Plate 11 - Pattern 1-4 - Scatterometry Target 
Plate 12 - Pattern 1-4 - Scatterometry Target. (top) GDS II drawing of one of the smaller patterns of 1-4 at a reduced FOV. (bottom) SEM micrograph of a similar area. 


\section{0um x 150um}
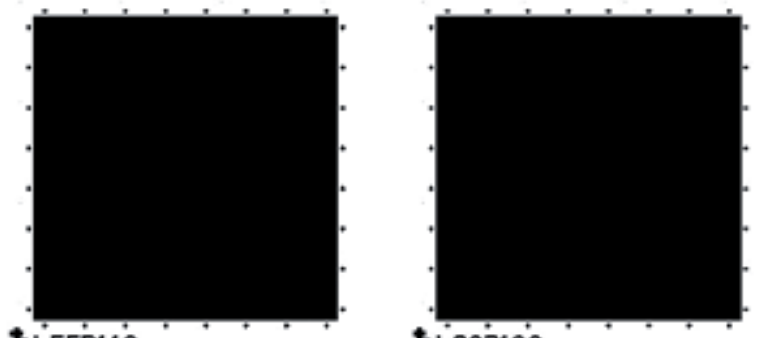

L55P110

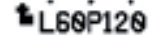

1
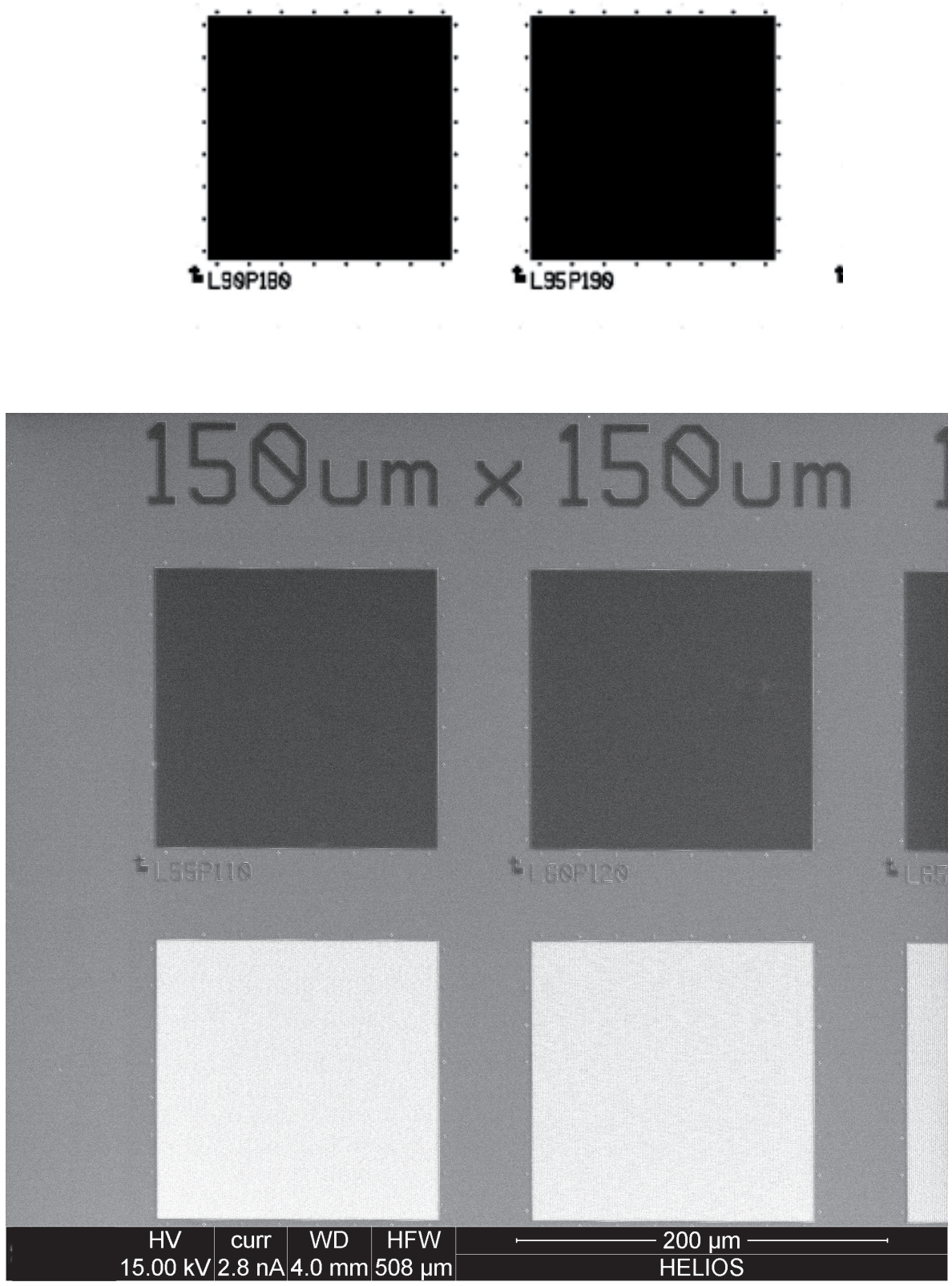

Plate 12 - Pattern 1-4 - Scatterometry Target 
Plate 13 - Pattern 1-4 - Scatterometry Target. (top) GDS II drawing of one of the 150 x $150 \mu \mathrm{m}$ patterns of 1-4 at a reduced FOV. (bottom) SEM micrograph of a similar area. 

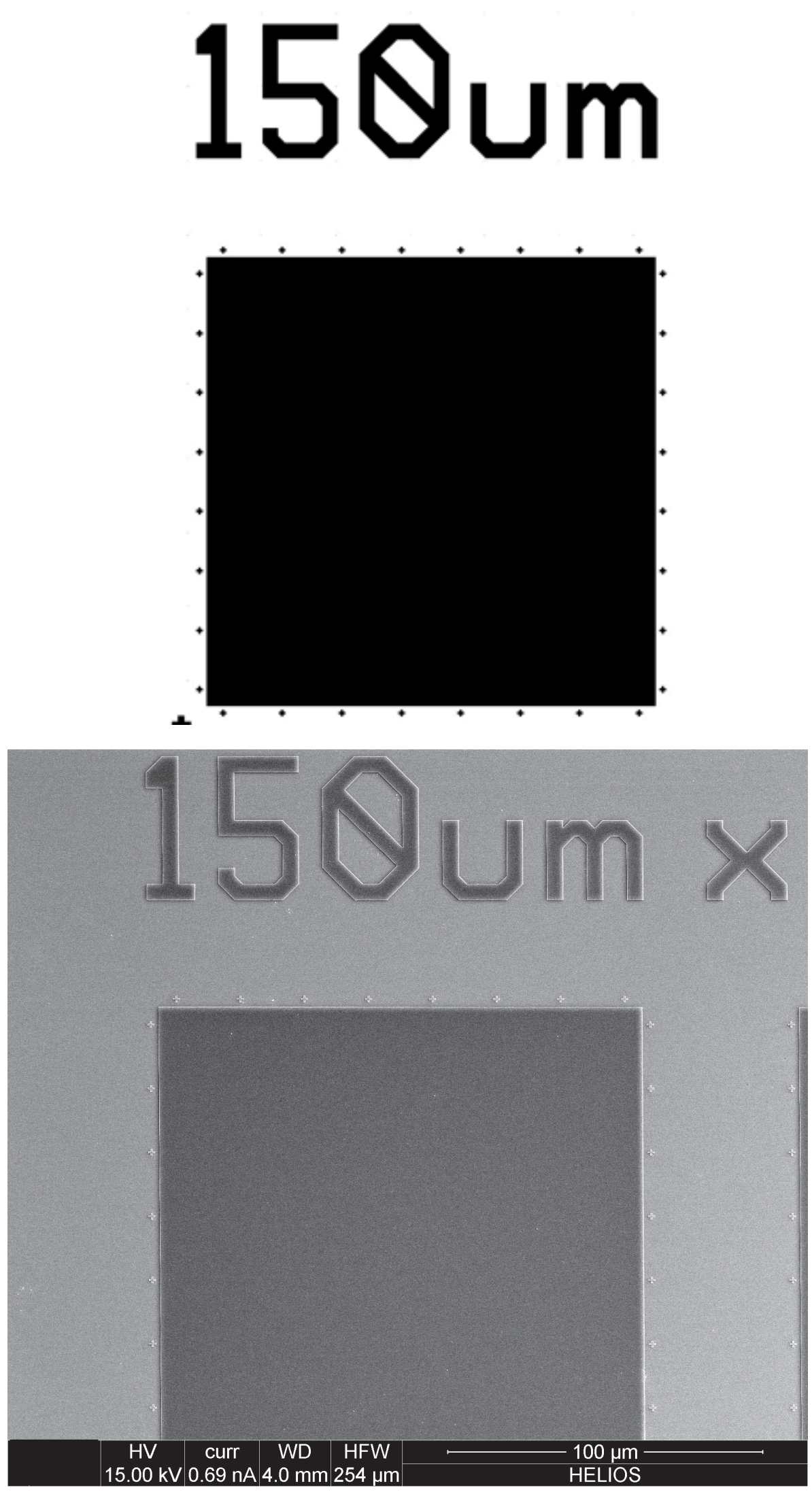

Plate 13 - Pattern 1-4 - Scatterometry Target 
Plate 14 - Pattern 1-5 - Line edge roughness. (top) GDS II drawing of Pattern 1-5 at a wide field of view. (bottom) SEM Micrograph of a similar area.

\section{Explanation:}

Line Edge Roughness (LERNIST). NIST designed line space arrays with built in periodic line edge roughness (LER). The amplitude and the frequency (wavelength) of the intentional edge roughness pattern is varied across a set of parameters. The values noted are the nominal linewidth and roughness periodicity, design values, which after the IC fabrication process changes somewhat. Some of the intentional changes fade into the "noise" caused by other characteristic process and material-dependent effects. For example, depending on the grain size of the polysilicon or properties of the photoresist and exposure/develop process, there is an unavoidable random roughness of all lines. Some of the lines have intentional edge only on one side, others might have other variations. These are depicted with identifiers and is well visible on SEM images. These are designed in $150 \mu \mathrm{m}$ square pads with $100 \mu \mathrm{m}$ space, ideal for OCD or CDSAXS experiments on such periodic roughness. Tabs are 1/2 of periodicity.

Series 4 - 6: L100P300: Amp 5-35 (5 nm steps) / Per 110; L150P450: Amp 20, 40, 60 / Per 200

Series 7: L150P450: Amp 20, 40, 60 / Per 200

Series 4 has alternating tabs on left/right side of line

Series 5 has double aligned tabs on left/right side of line

Series 6 has alternating tabs on left/right side of line; but lines are staggered

Series 7 has tabs only on one side 
NIST SERIES 4 CENTERLINE FLUCTUATION LINEWIDTH CONSTANT
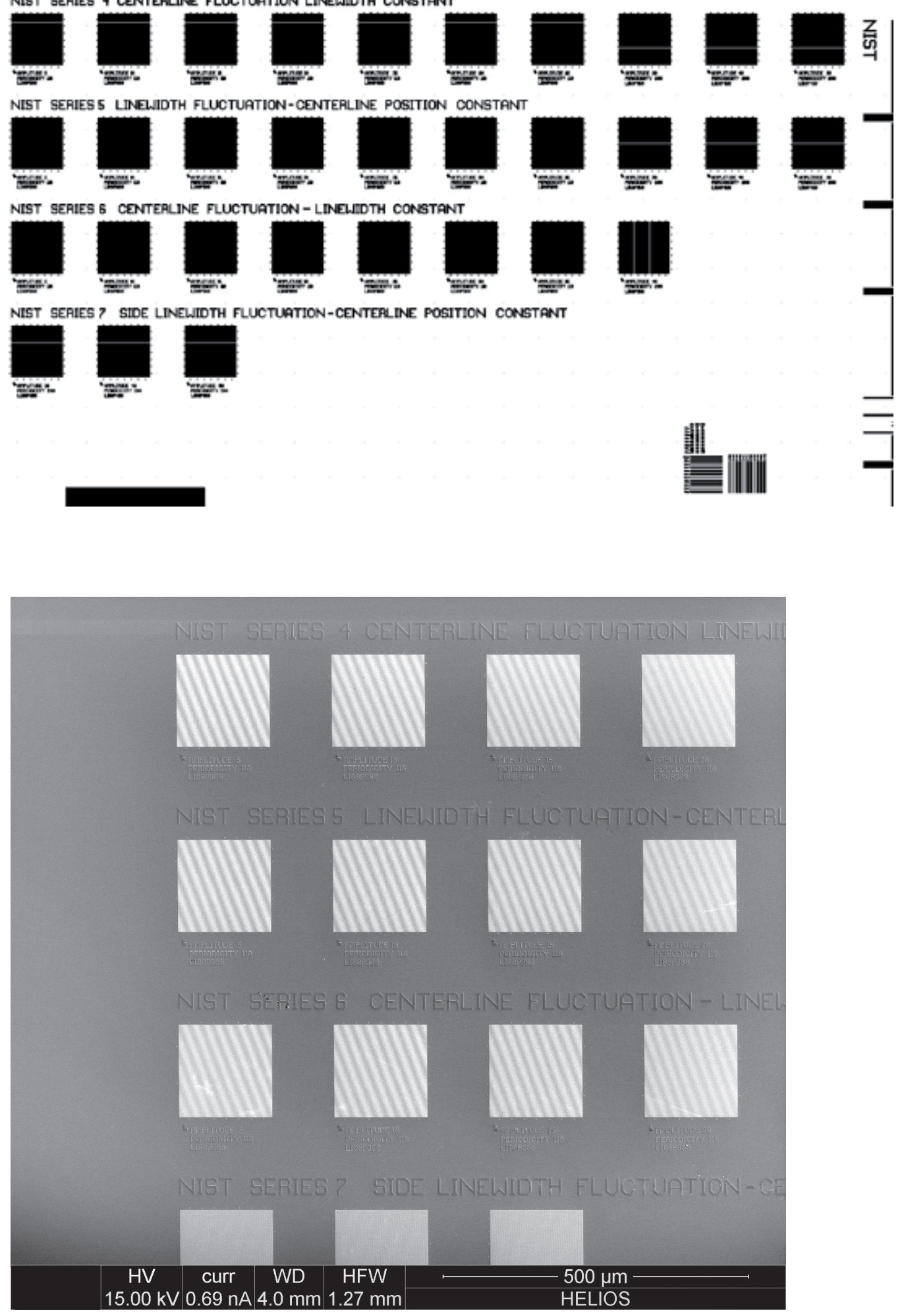

Plate 14 - Pattern 1-5 - Line edge roughness 
Plate 15 - Pattern 1-5 - Line edge roughness. (top) GDS II drawing of Pattern 1-5 at a smaller field of view. (bottom) SEM micrograph of a similar area. 

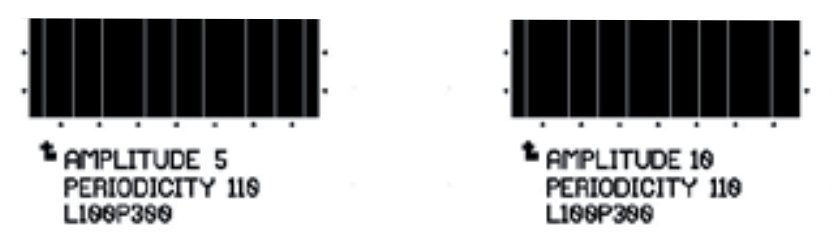

\section{NIST SERIES 6 CENTE}
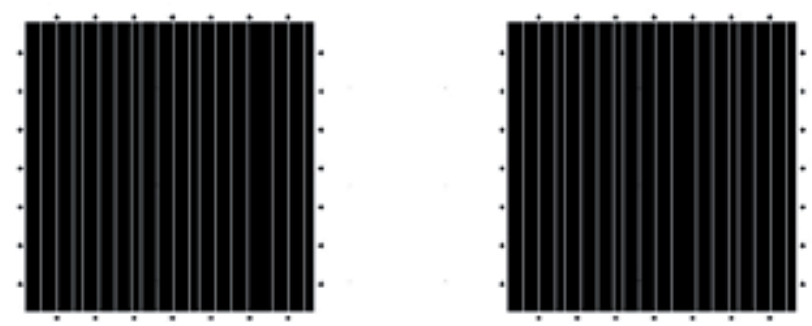

t mplitude 5

PERIODICITY 110

L180P380

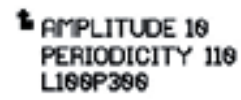

NIST SERIES 7 SIDE L

NIST SERIES 6 CENTERLINE
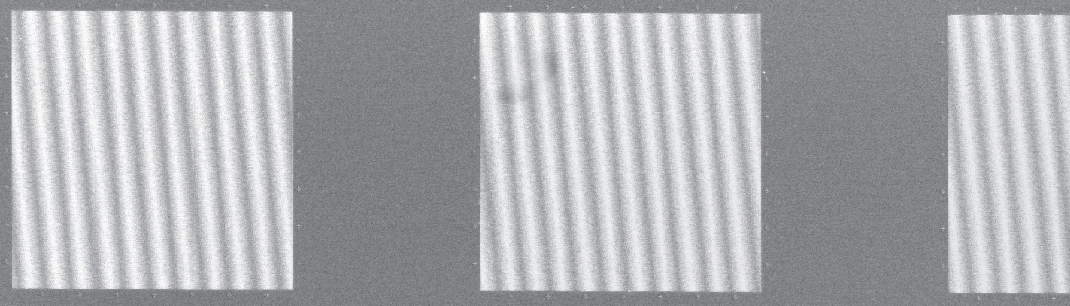

NIST SERIES ?

SIDE LINEWID

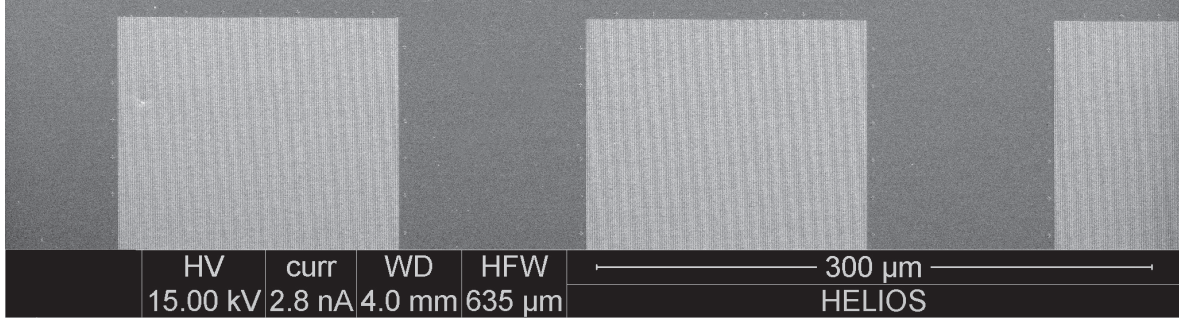

Plate 15 - Pattern 1-5 - Line edge roughness 
Plate 16 - Pattern 1-5 - Line edge roughness. (top) Medium magnification SEM image of Pattern 1-5. (bottom) Smaller HFW SEM micrograph of a similar area. 

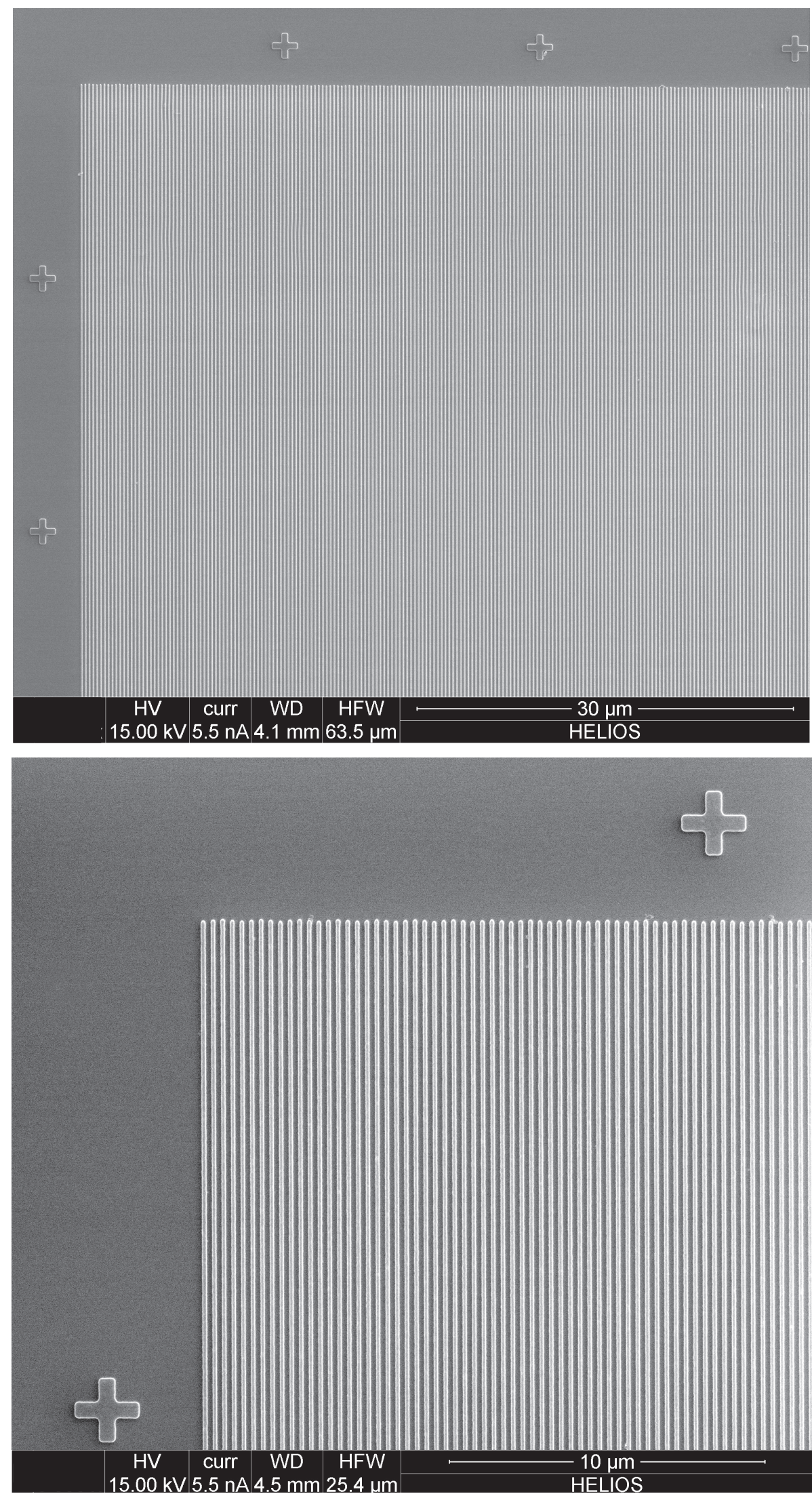

Plate 16 - Pattern 1-5 - Line edge roughness 
Plate 17 - Pattern 1-6 - Scatterometry Varied. (top) GDS II drawing of Pattern 1-6 at a wide field of view. (bottom) SEM micrograph a similar area.

\section{Explanation:}

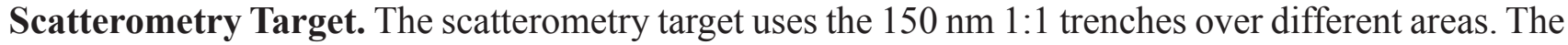
following is a list of the different areas covered by the $140 \mathrm{~nm}$ trench: 100x100 $\mu \mathrm{m}, 90 \times 90 \mu \mathrm{m}, 80 \times 80$ $\mu \mathrm{m}, 70 \mathrm{x} 70 \mu \mathrm{m}, 60 \times 60 \mu \mathrm{m}, 50 \times 50 \mu \mathrm{m}, 40 \times 40 \mu \mathrm{m}, 30 \times 30 \mu \mathrm{m}, 20 \times 20 \mu \mathrm{m}$, and 10x10 $\mu \mathrm{m}$. 

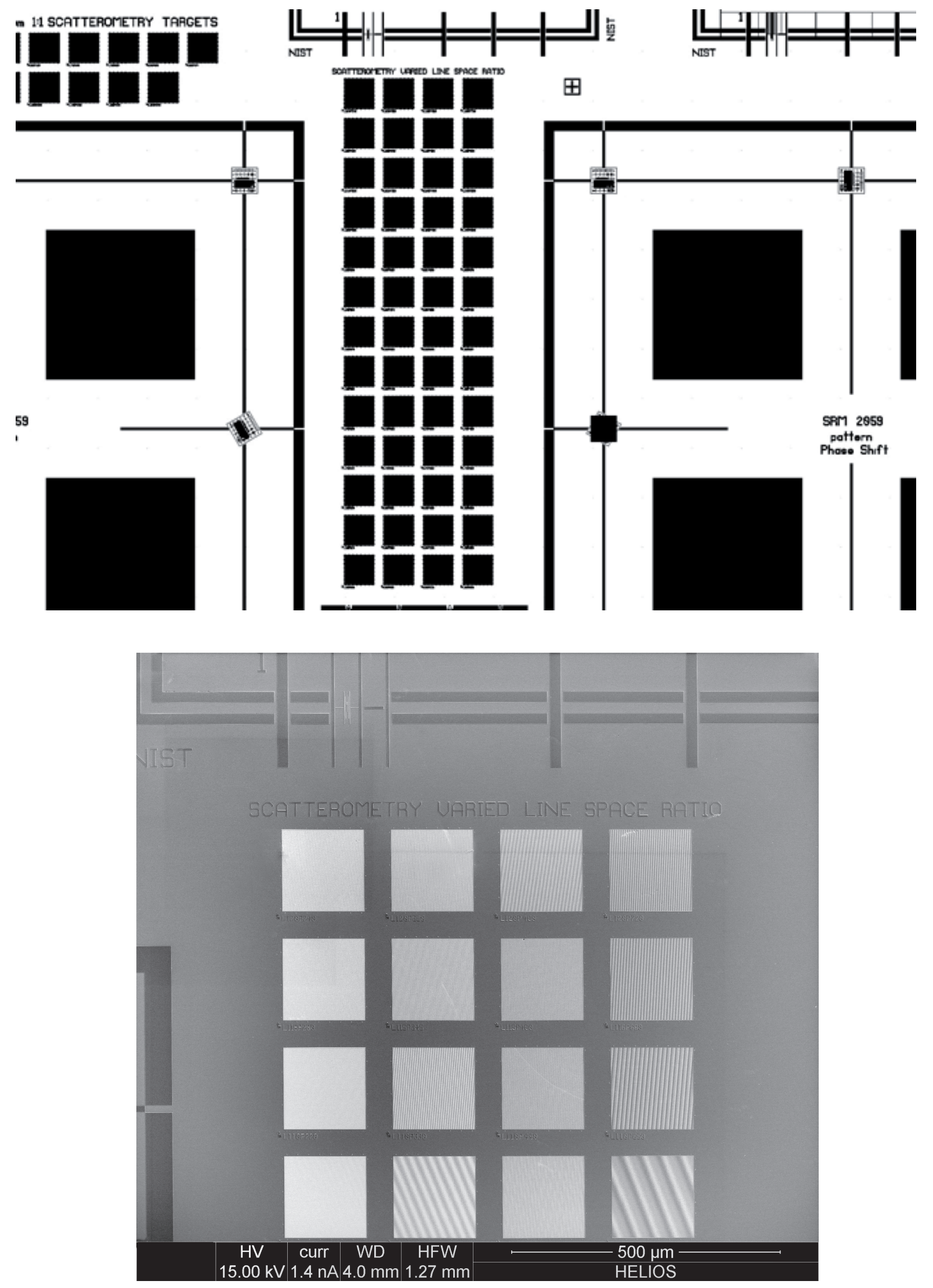

Plate 17 - Pattern 1-6 - Scatterometry Varied 
Plate 18 - Pattern 1-6 - Scatterometry Varied. (top) GDS II drawing of Pattern 1-6 at a wide field of view. (bottom) SEM micrograph a similar area of Pattern 1-6. 
| | | |

I

\section{SCATTEROMETRY UARIED LINE !}
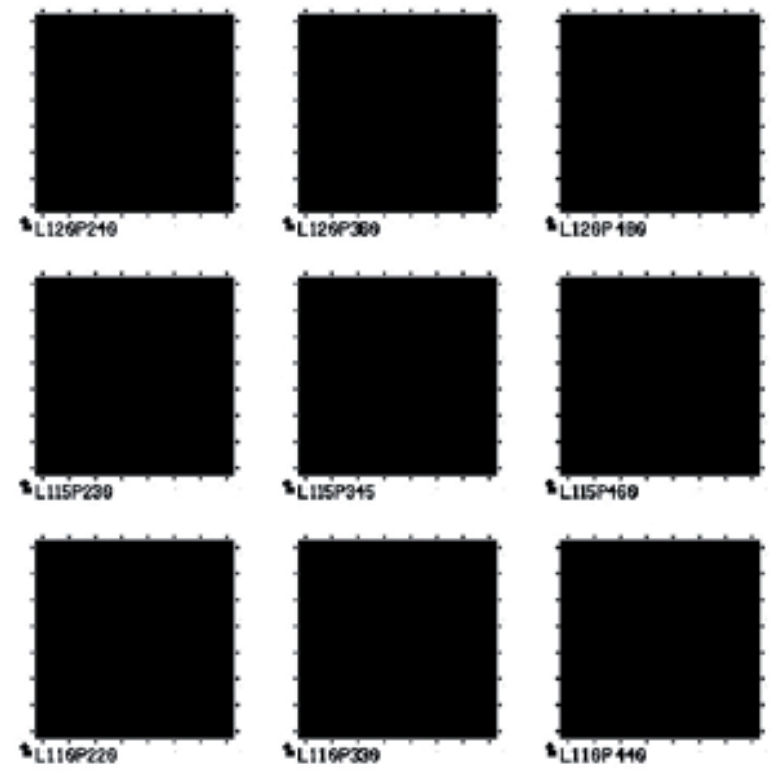

tLi16esos

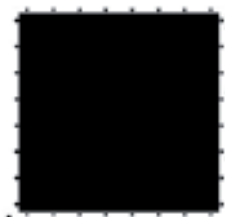

ธL116P410
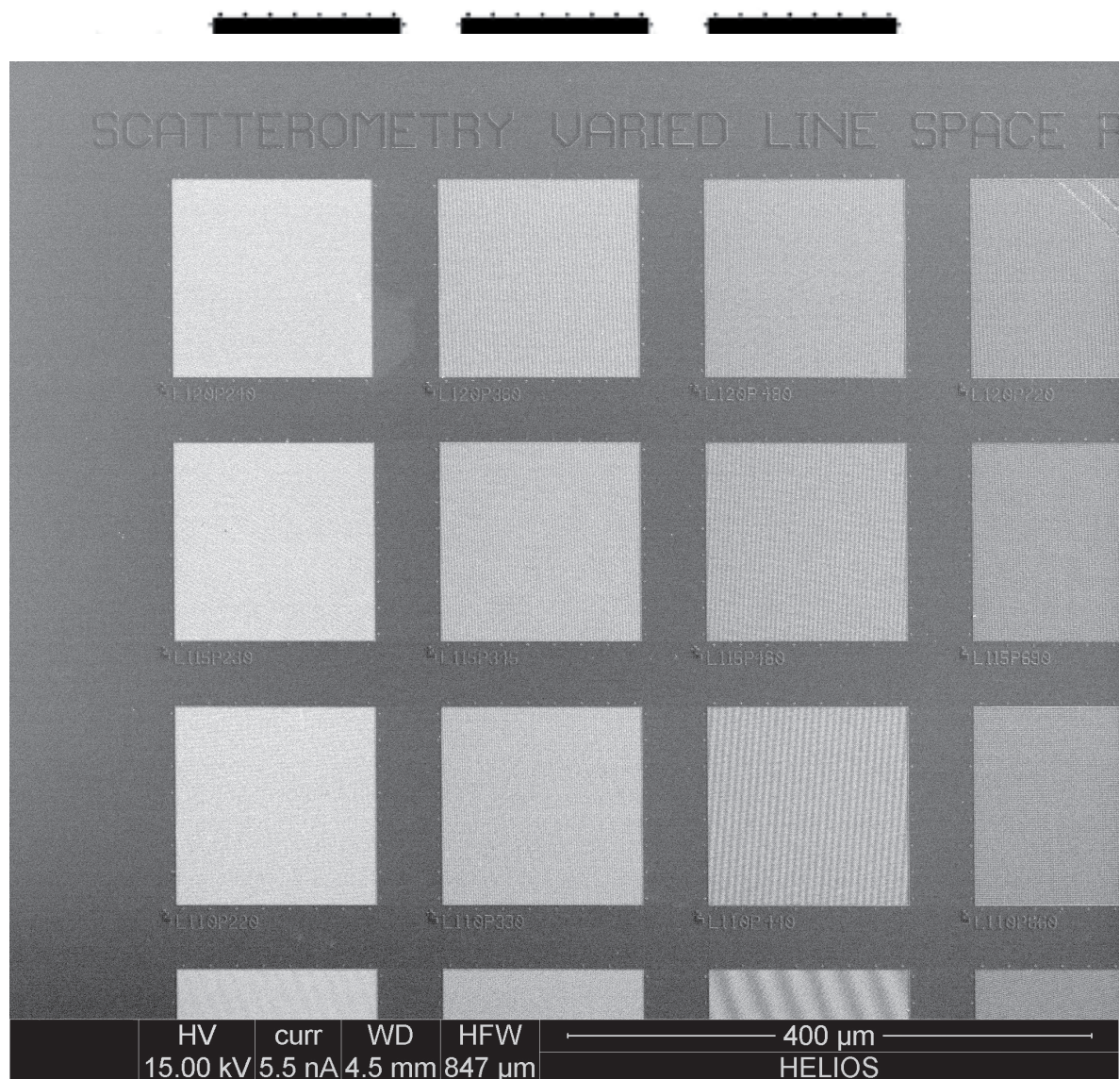
Plate 19 - Pattern 1-6 - Scatterometry Varied. (top) GDS II drawing of Pattern 1-6 at a wide field of view. (bottom) SEM micrograph a similar area of Pattern 1-6. 


\section{SCATTEROI}
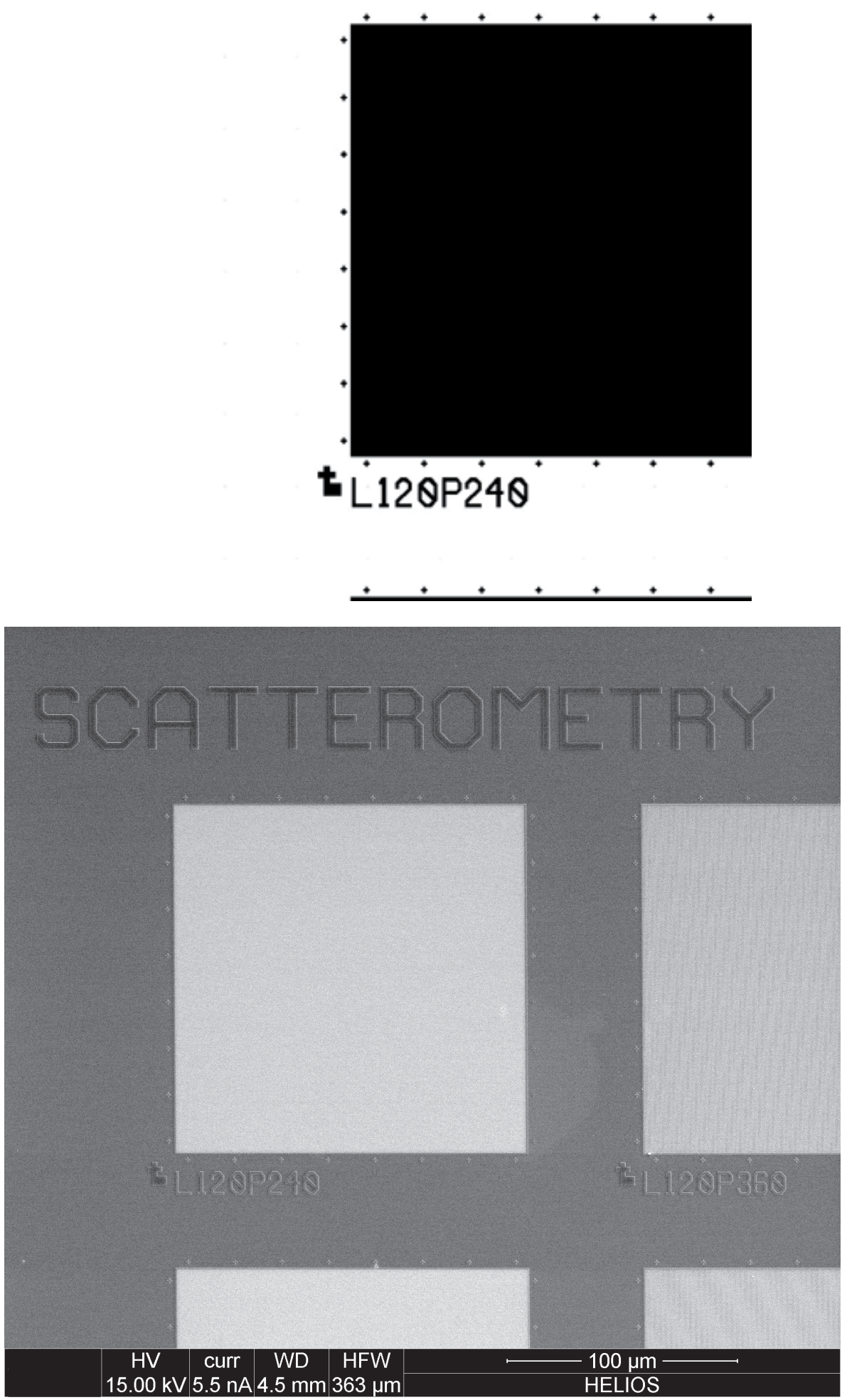

Plate 19 - Pattern 1-6 - Scatterometry Varied 
Plate 20 - Pattern 1-6 - Scatterometry Varied. (top) ) Higher magnification SEM micrograph of Pattern 1-6 at a low horizontal field width. (bottom) SEM micrograph a similar area of Pattern 1-6 at an even higher magnification and horizontal field of view. 

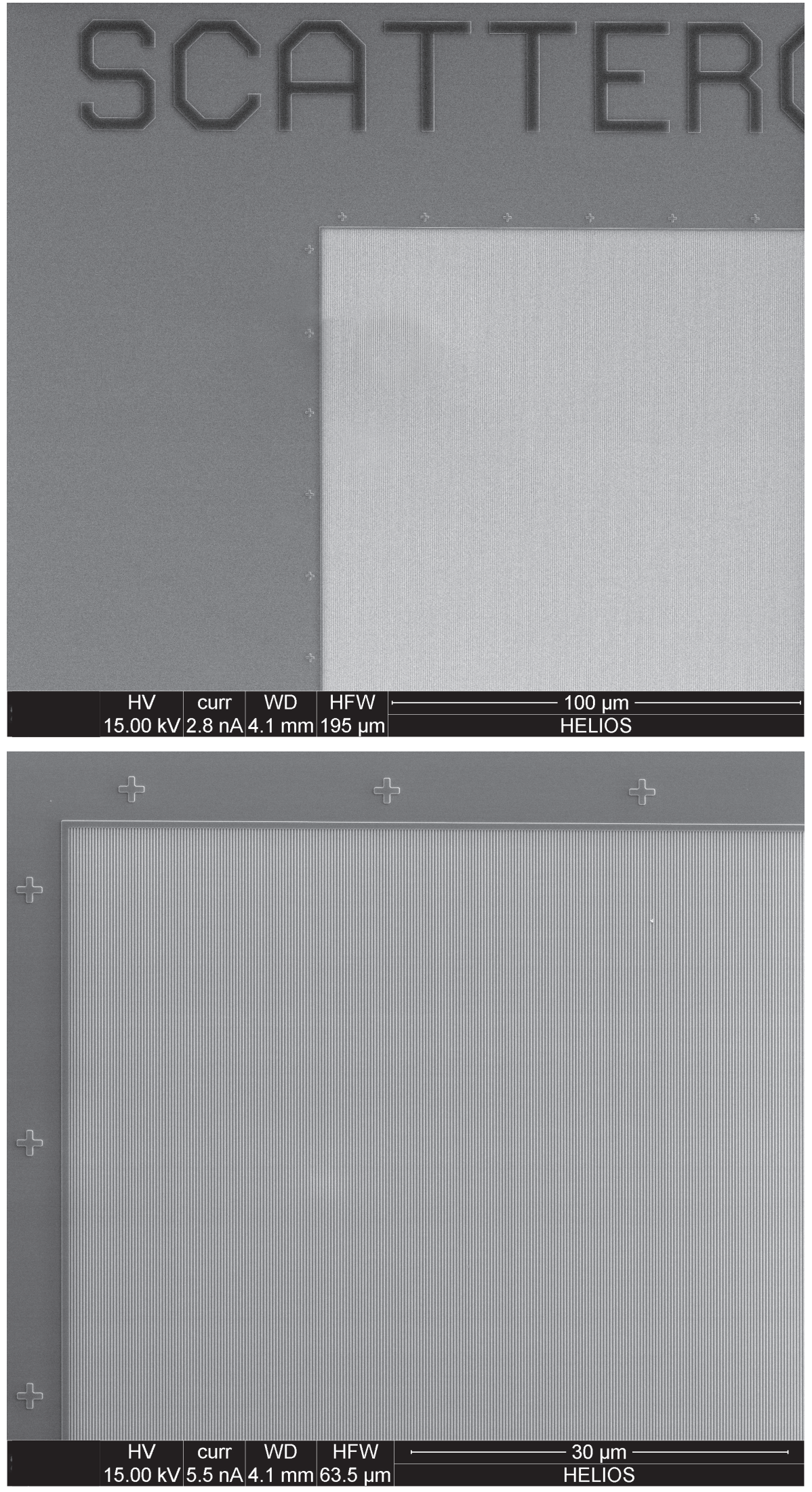

Plate 20 - Pattern 1-6 - Scatterometry Varied. 
Plate 21 - Pattern 1-7 - Via Pad Variation. (top) GDS II drawing of Pattern 1-7 at a wide field of view. (bottom) SEM Micrograph of a similar area.

\section{Explanation:}

Via Pad Varied Corner Radius. This module contains 150x150 $\mu \mathrm{m}$ blocks of vias that vary based on corner rounding. The following sizes indicate the rounded corner radius 5, 10, 15, 20, 25, 30, 35 $\mathrm{nm}$; the pad dimensions are: 100x150 $\mu \mathrm{m}$, Pitch: X: $200 \mu \mathrm{m}, \mathrm{Y}: 300 \mu \mathrm{m}$. 


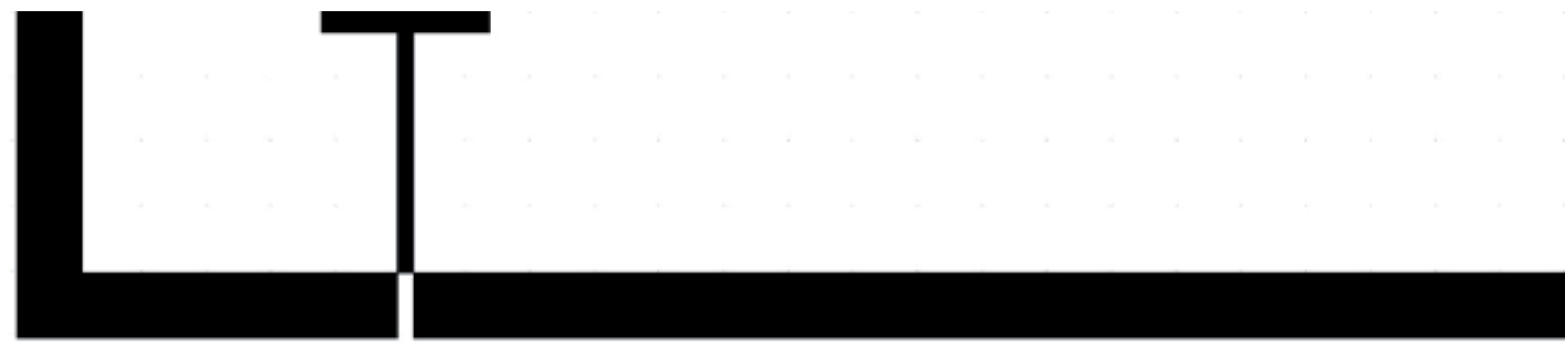

\section{$100 \times 150 N M$ UIA PAD WITH UARIATION IN CORNER RADIUS}
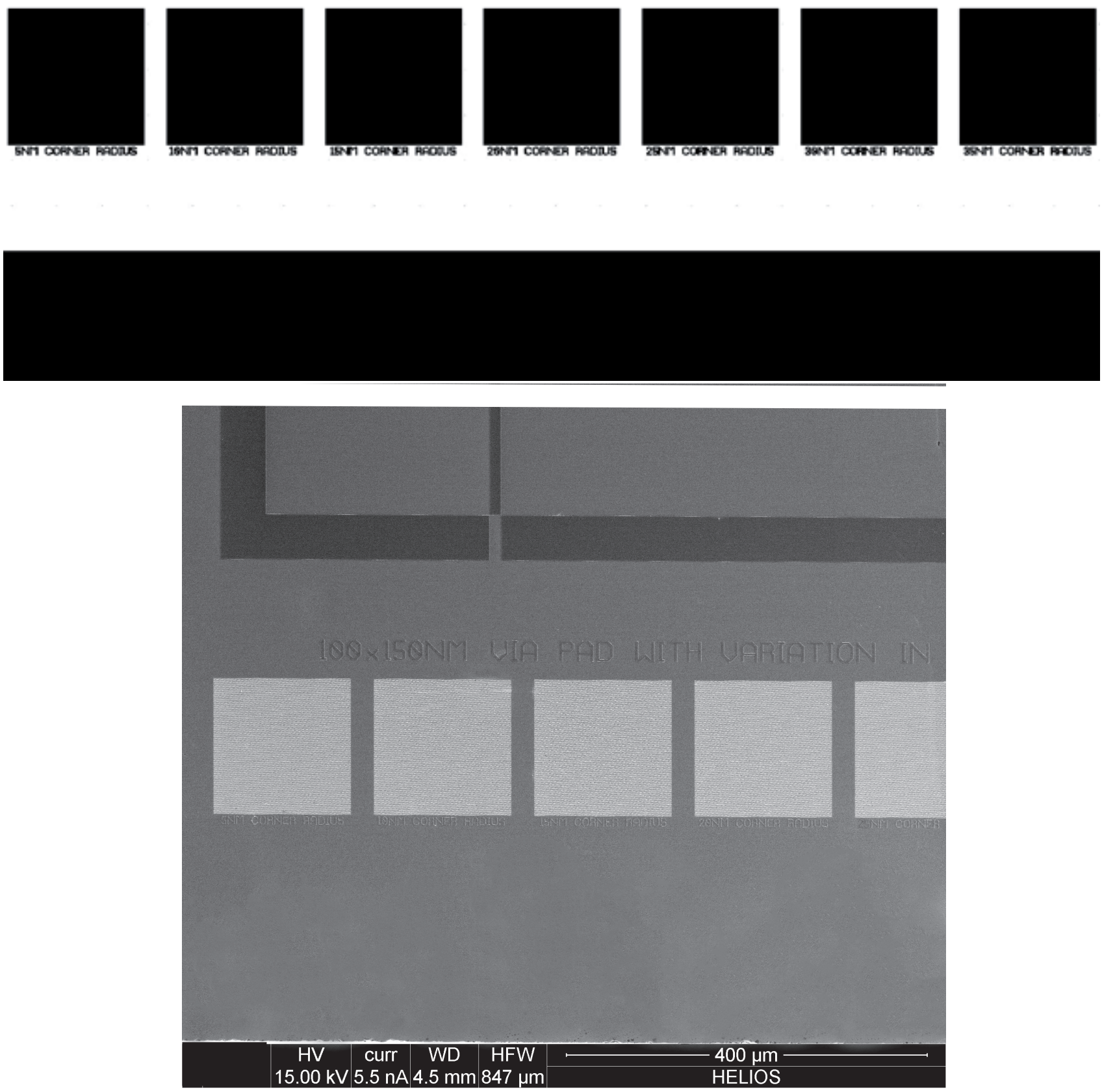

Plate 21 -Pattern 1-7 - Via Pad Variation 
Plate 22 - Pattern 1-7 - Via Pad Variation. (top) GDS II drawing of Pattern 1-7 at a wide field of view. (bottom) SEM Micrograph of a similar area. 


\section{$100 \times 150 \mathrm{NM} \mathrm{U}]$}

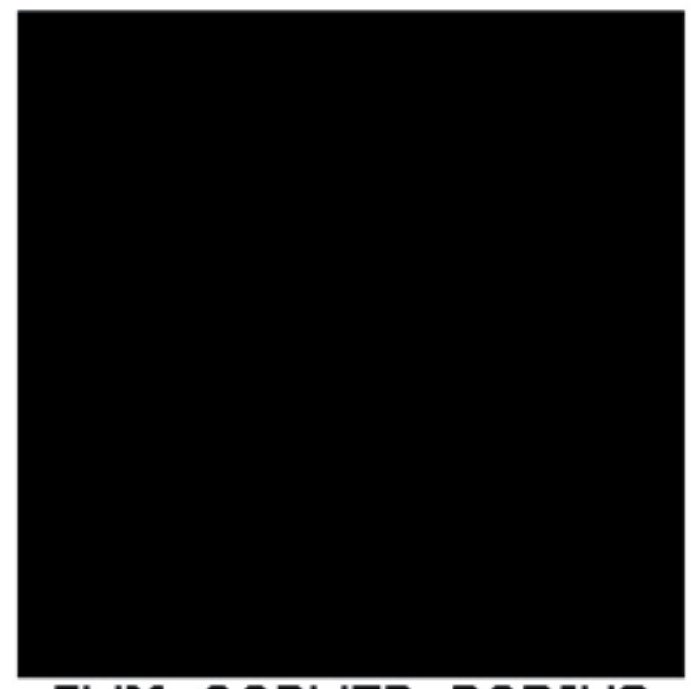

5NM CORNER RADIUS

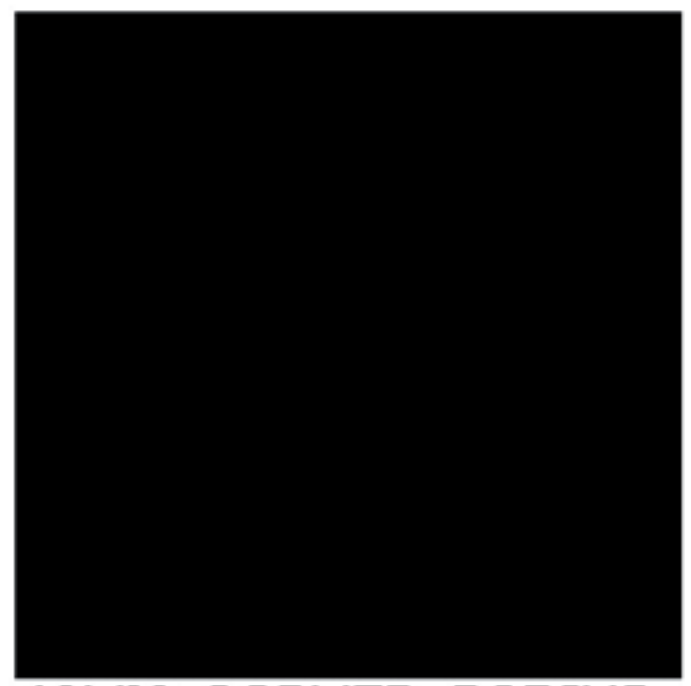

1ONM CORNER RADIUS

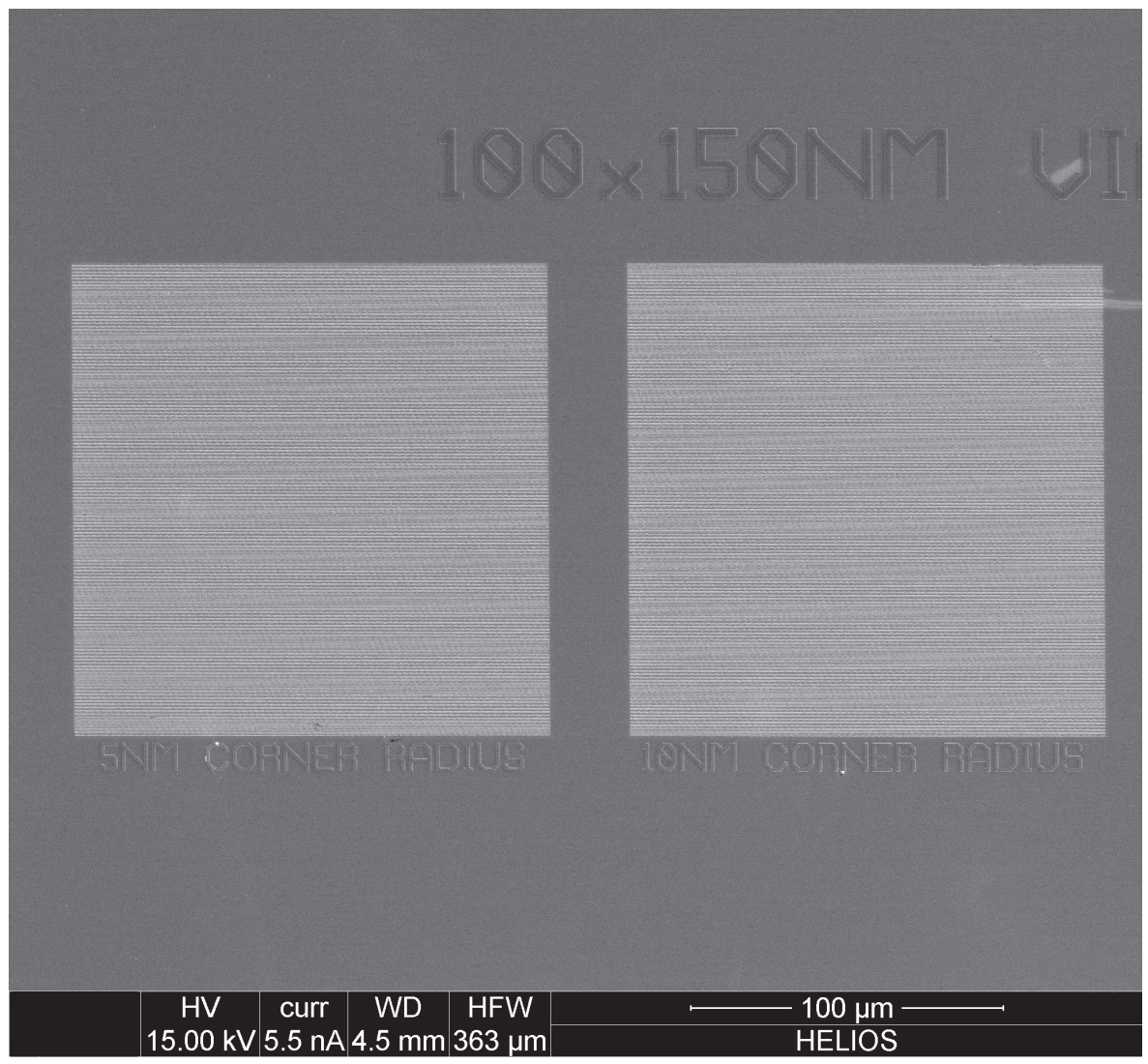

Plate 22 -Pattern 1-7 - Via Pad Variation 
Plate 23 - Pattern 1-7 - Via Pad Variation. (top) SEM micrograph taken of $10 \mathrm{~nm}$ corner radius and (bottom) SEM micrograph of $5 \mathrm{~nm}$ of corner radius. 

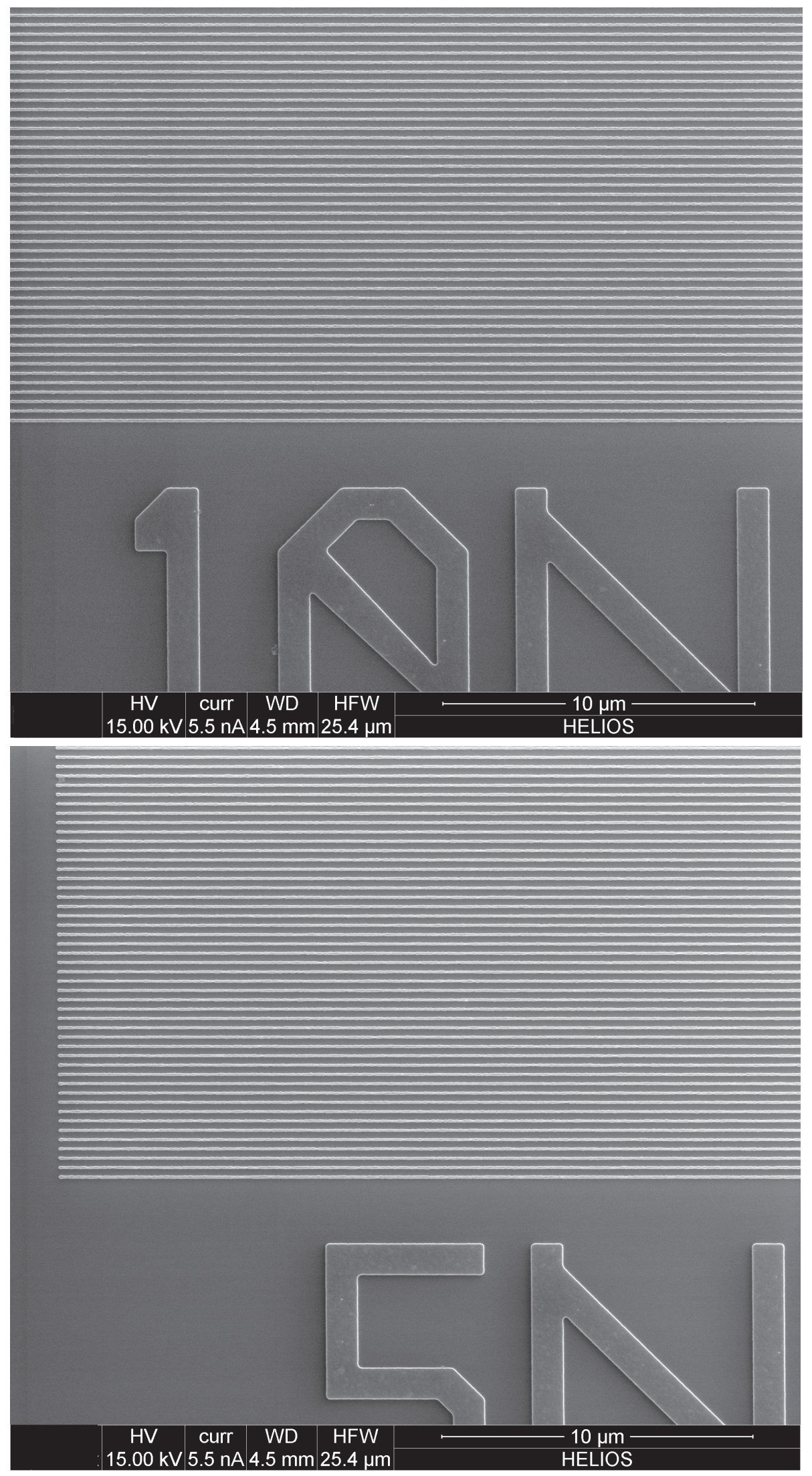

Plate 23 - Pattern 1-7 - Via Pad Variation 
Plate 24 - Pattern 1-8 - RM 8090. (top) GDS II drawing of Pattern 1-8 at a wide FOV. (bottom) GDS II drawing of one of the smaller Patterns of 1-8 at a smaller FOV.

[The RM 8820 patterns found adjacent to this pattern have been discussed earlier in this document and will not be repeated here.]

\section{Explanation:}

NIST pattern for NIST RM 8090. Several RM 8090 structures were added to the RM 8820 reticle. Within this section there are two RM 8090 structures, regardless of size, one is completely phase shifted and the other is not. In the NIST quad section, there are six RM 8090 structures, two are standard phase shift modules, but one is grounded, and the other four are 1/4 the size of the original. Two of the 1/4 size are line/space modules and two are trench modules; one of the line/space modules is grounded.

[The original RM 8090 pattern was originally designed by Michael Postek and Andras Vladar and its history is described earlier in the text.] 

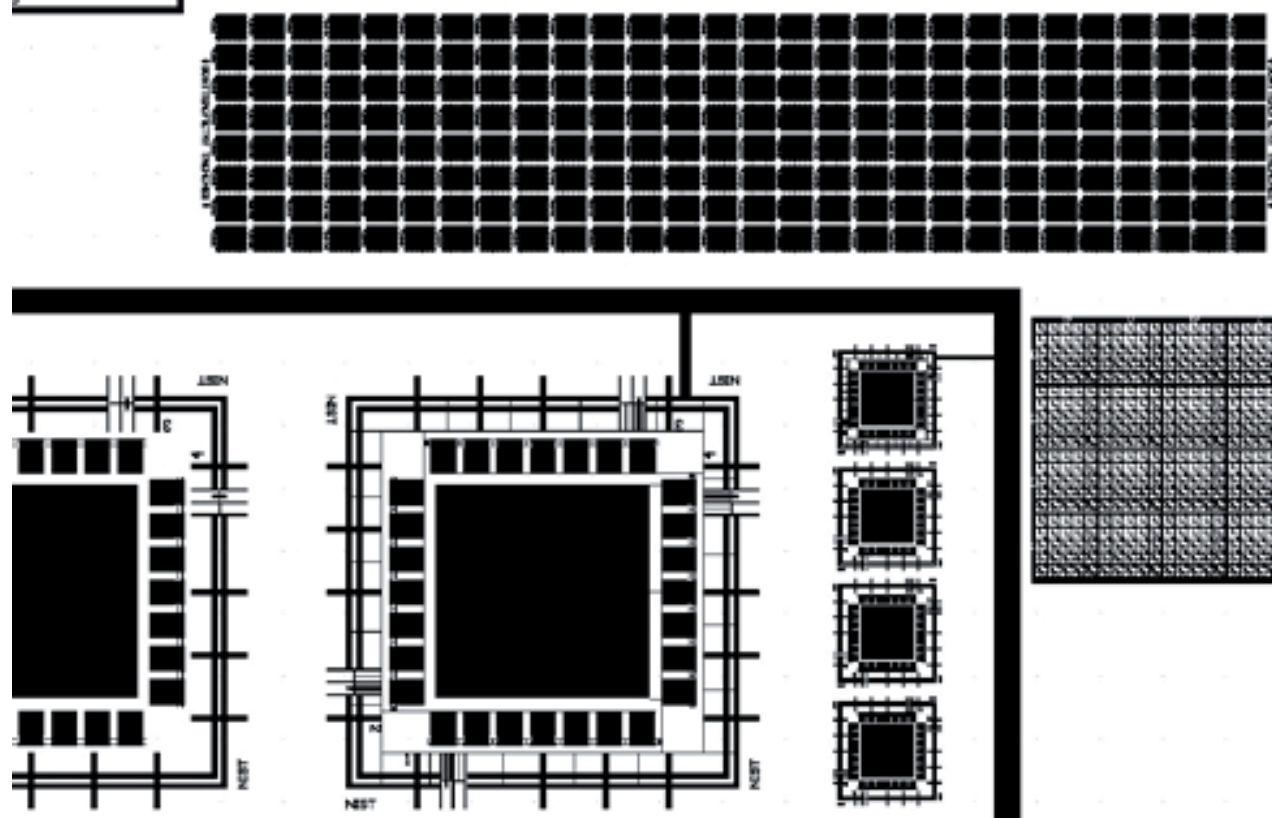

5

田

田
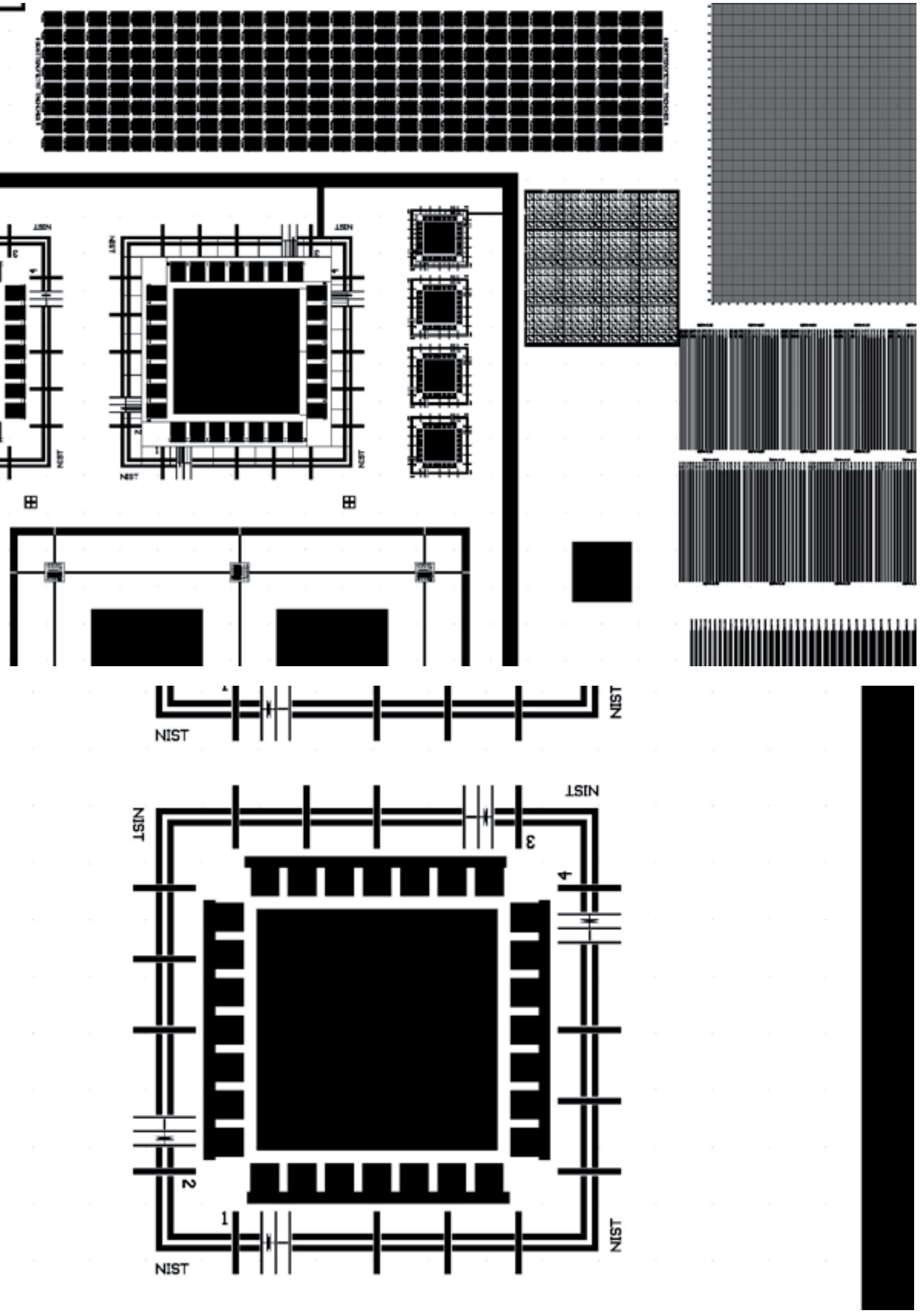

Plate 24 - Pattern 1-8 - RM 8090. 
Plate 25 - Pattern 1-8 - RM 8090/2090. SEM images of Pattern 1-8 at a wide FOV. (bottom) and one of the smaller Patterns of 1-8 at smaller FOV. 

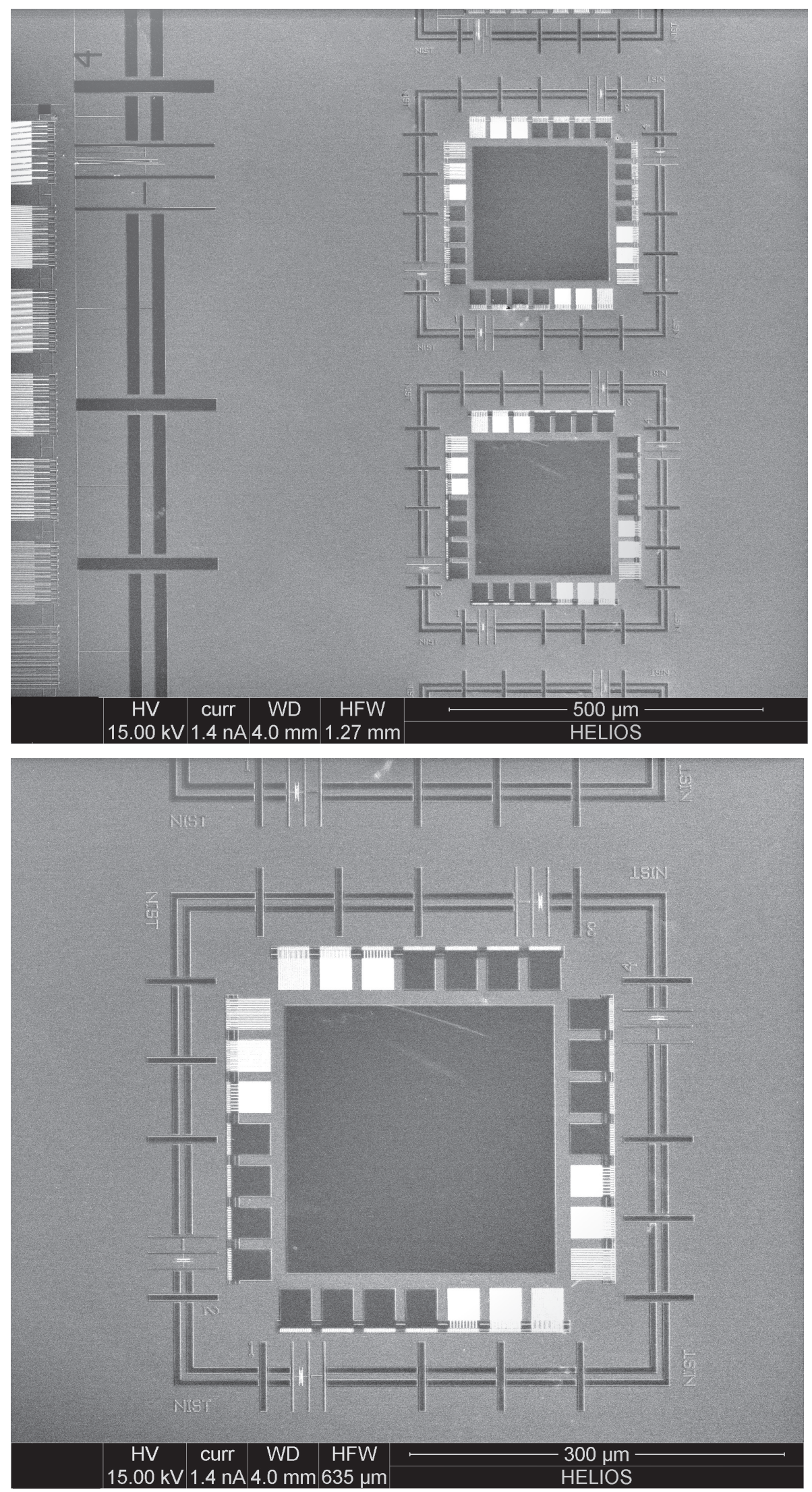

Plate 25 - Pattern 1-8 - RM 8090. 
Page intentionally left blank 
SECTION 2 
Plate 26 - Section 2-0 - Overall. (top) GDS II line drawing of the entire RM 8820 pattern highlighting Section 2 with the 6 additional major sub-patterns numerically identified. (bottom) GDS II detail drawing of Section 2 showing the 6 additional major sub-patterns included in this section. 


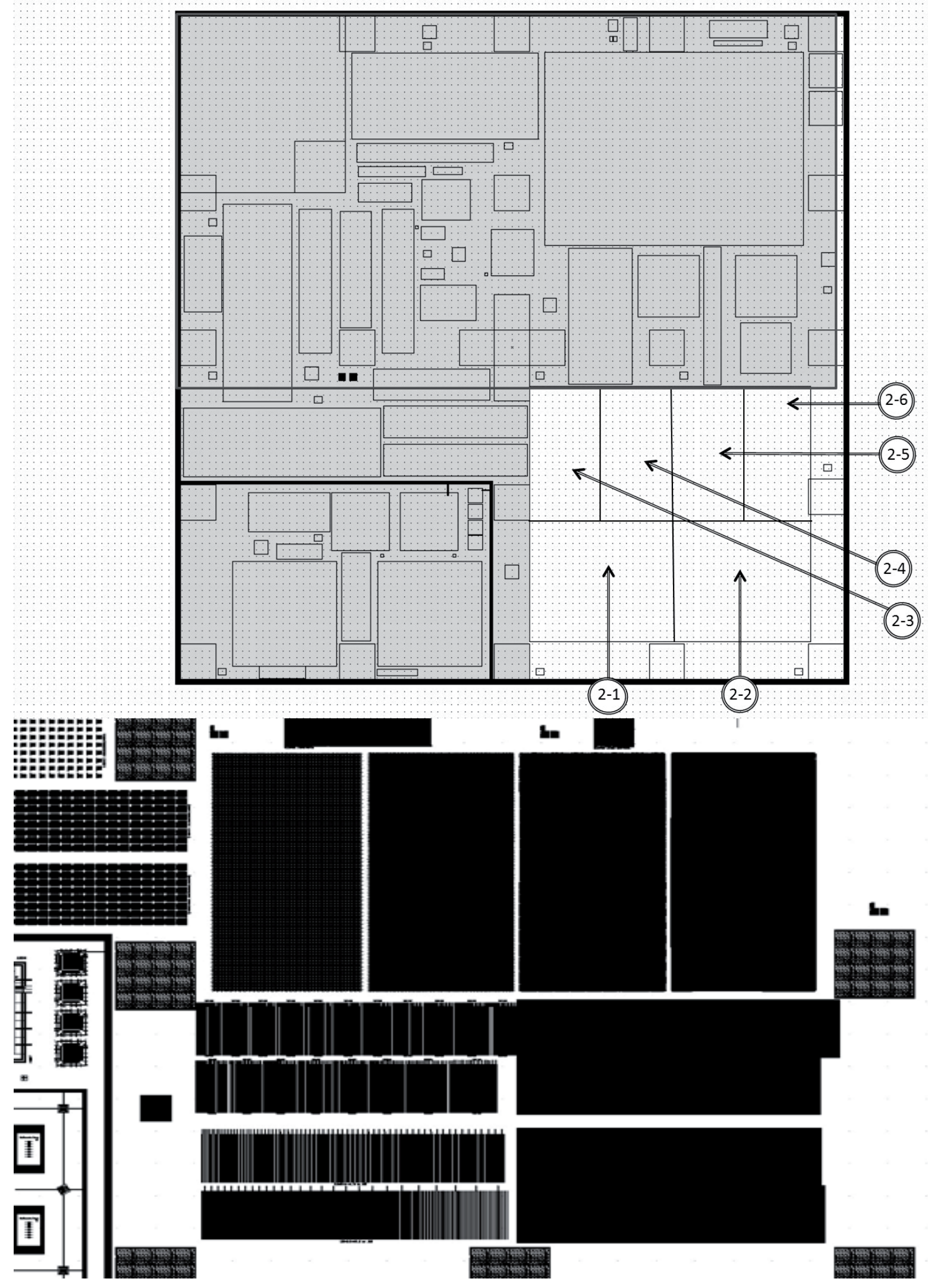

Plate 26 - Section 2. 
Plate 27 - Pattern 2-1 - Multiple Gap Lines Positive. (top) GDS II drawing of Pattern 2-1 at a wide FOV. (bottom) SEM micrograph of a similar area. 

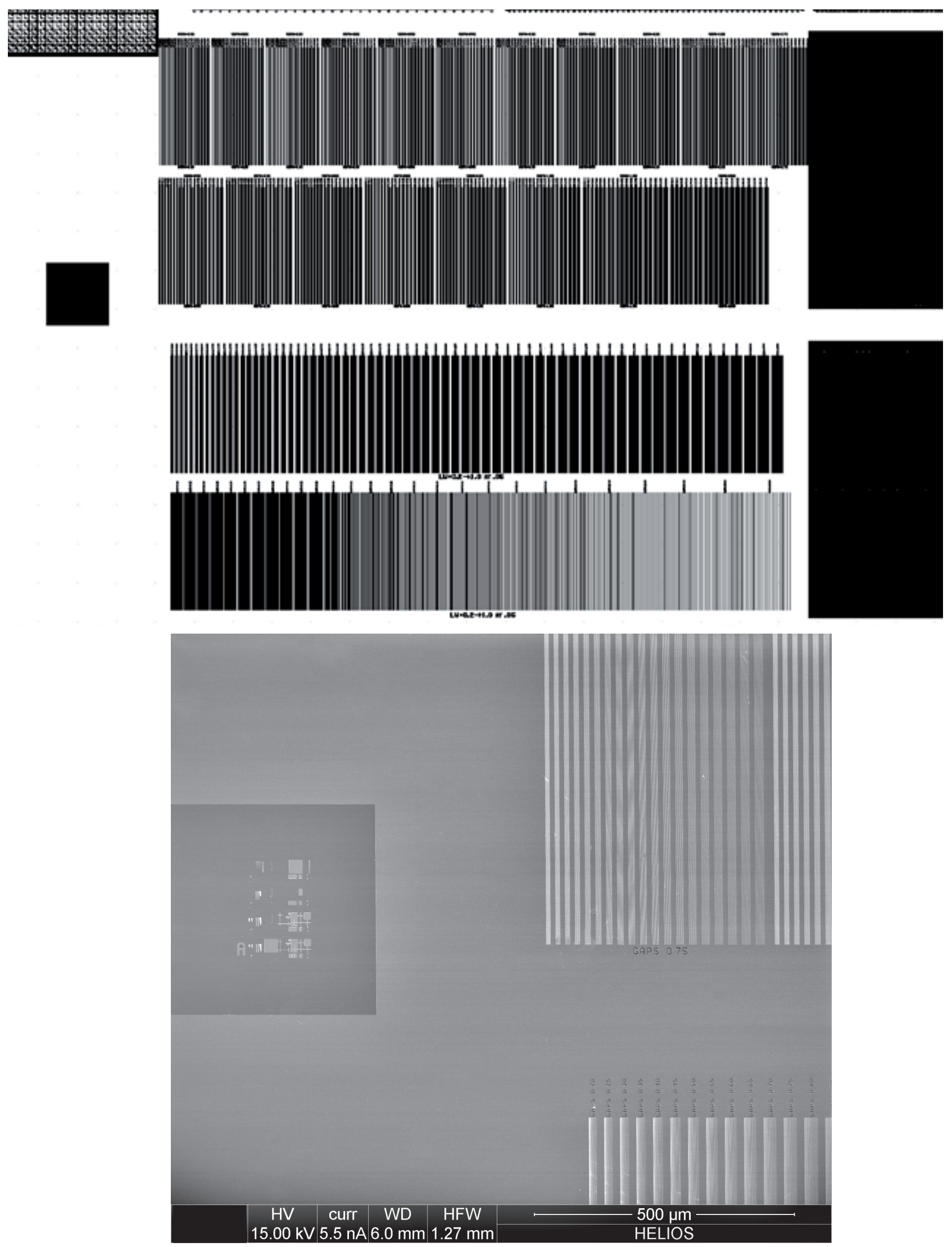

Plate 27 - Pattern 2-1 - Multiple Gap Lines Positive 
Plate 28 - Pattern 2-1 - Multiple Gap Lines Positive. (top) GDS II drawing of Pattern 2-1 at reduced FOV. (bottom) SEM micrograph of a similar area. 

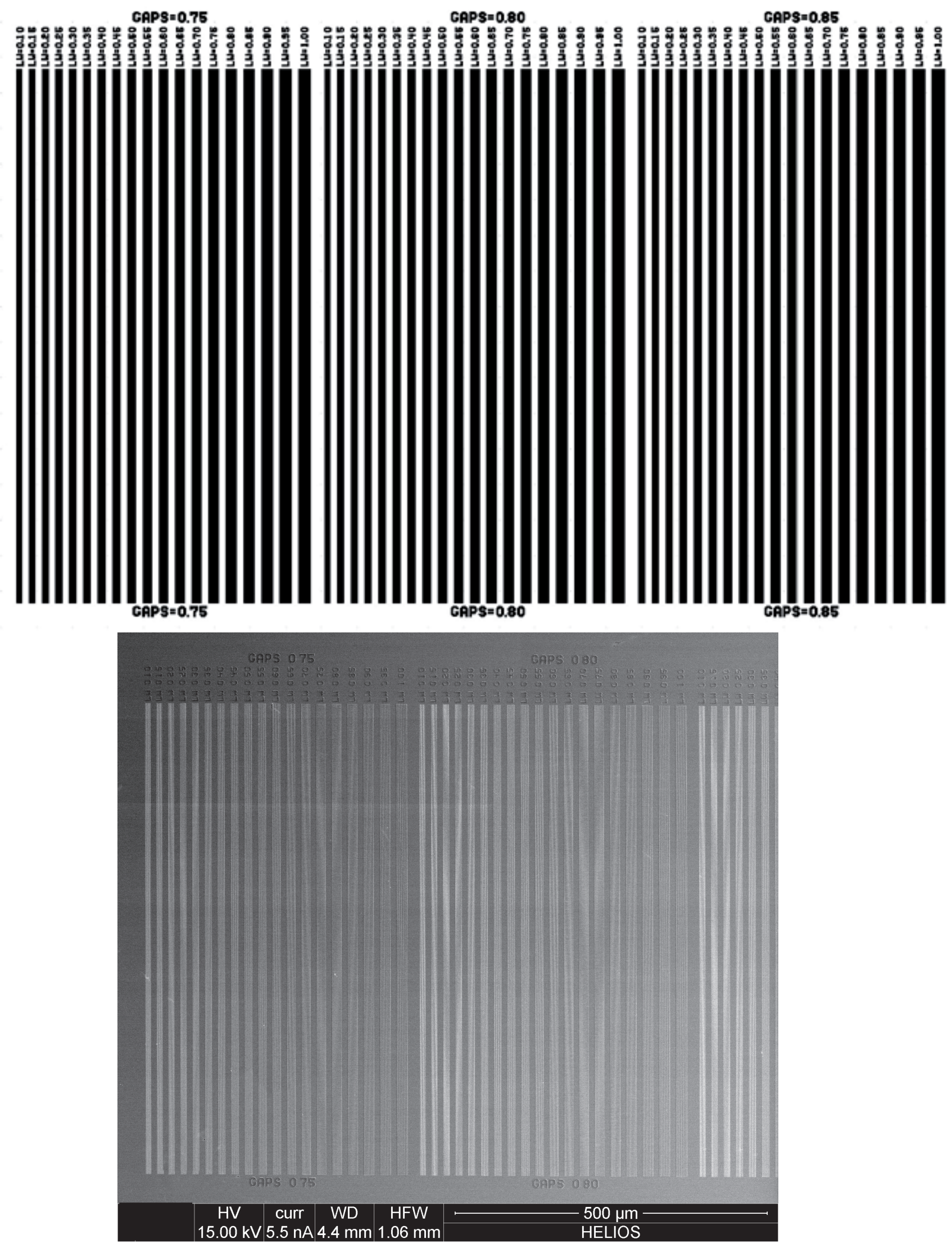

Plate 28 - Pattern 2-1 - Multiple Gap Lines Positive. 
Plate 29 - Pattern 2-1 - Multiple Gap Lines Positive. (top and bottom) SEM Micrographs of Pattern 2-1 at two different horizontal fields of view. 

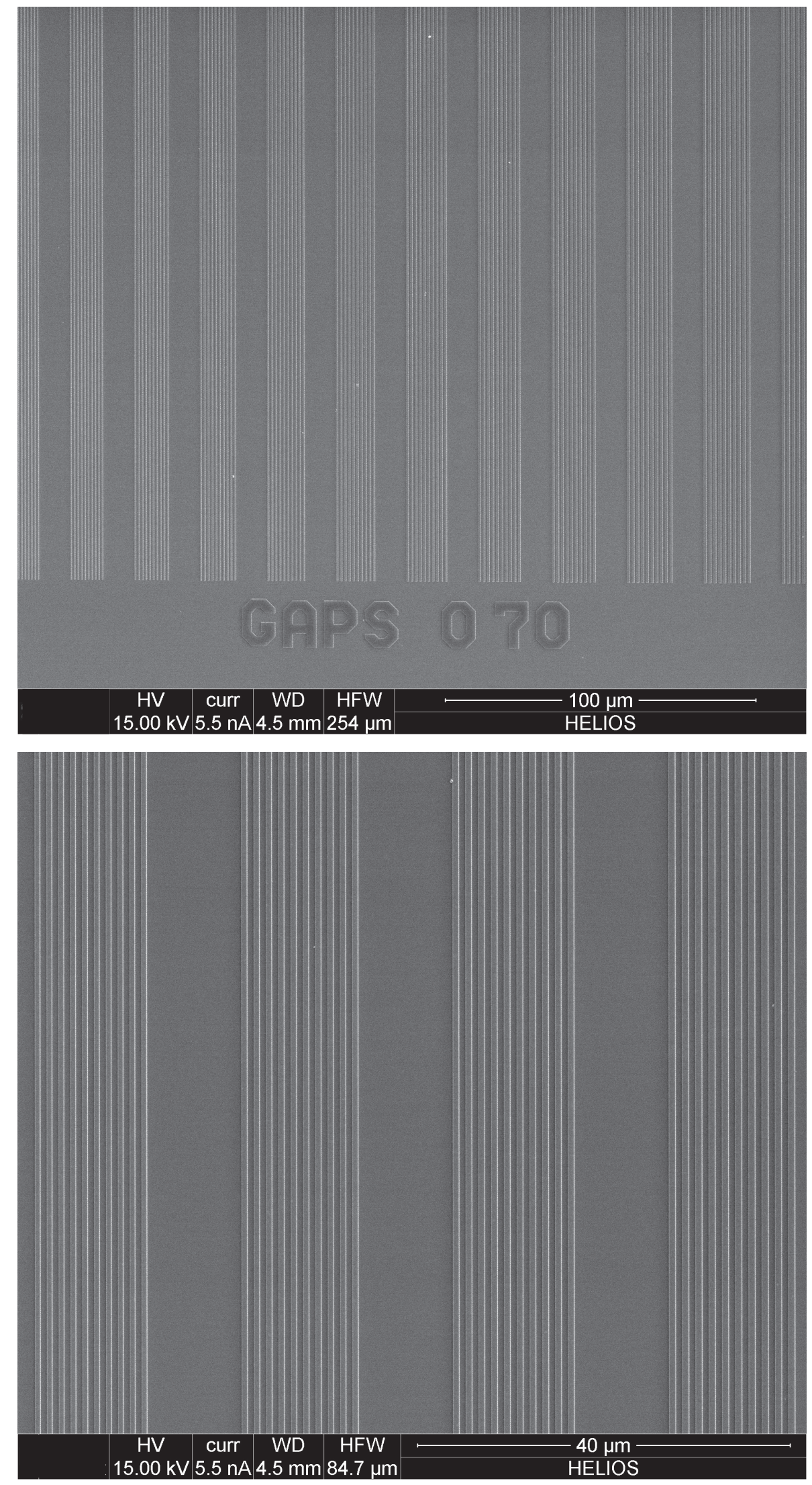

Plate 29 - Pattern 2-1 - Multiple Gap Lines Positive. 
Plate 30 - Pattern 2-2 - Multiple Gap Lines Negative. (top) GDS II drawing of the interface between the positive 2-1 pattern (left side) and the negative 2-2 pattern at a wide FOV. (bottom) SEM image of Pattern 2-2 at reduced HFW. 

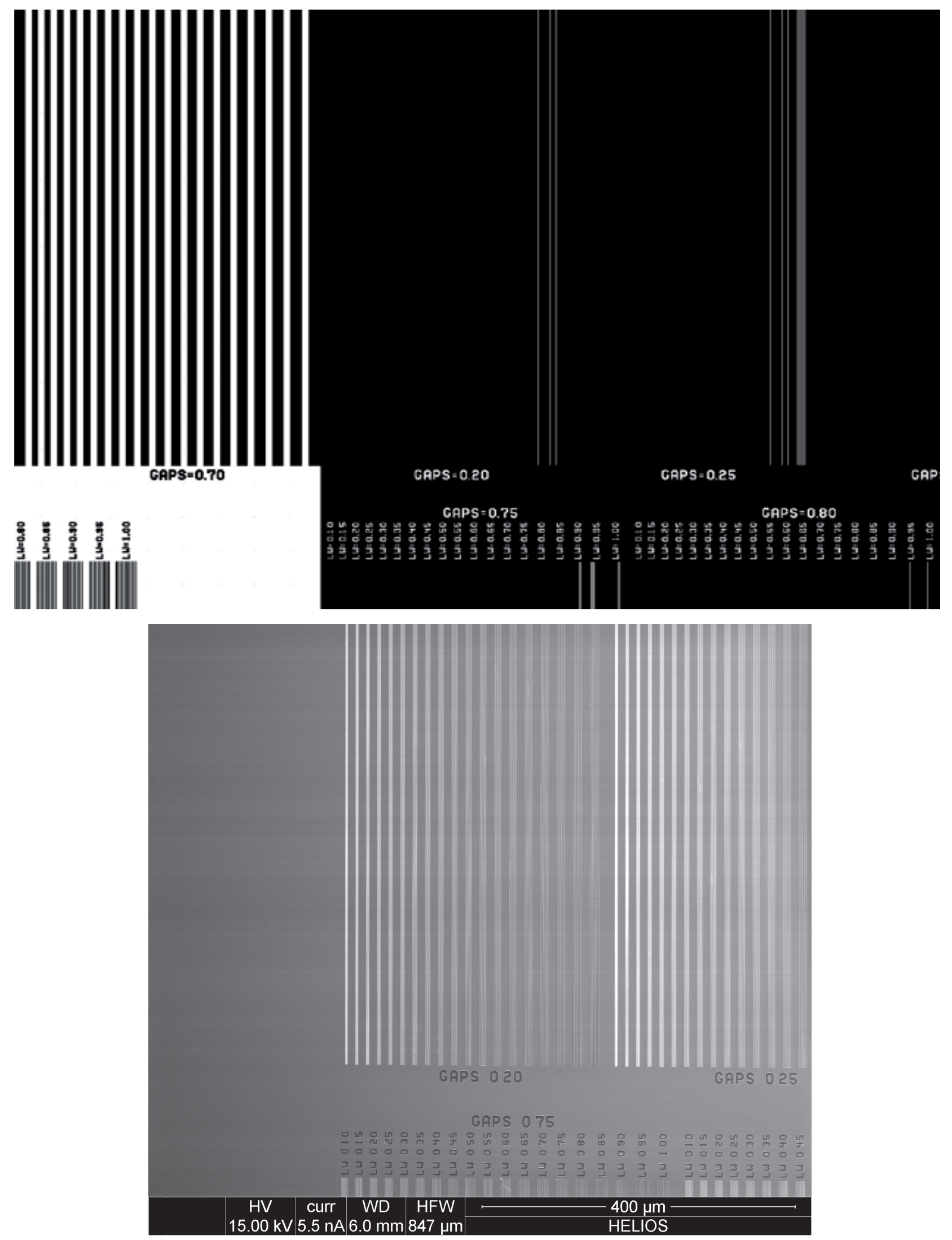

Plate 30 - Pattern 2-2 - Multiple Gap Lines Negative 
Plate 31 - Pattern 2-2 - Multiple Gap Lines Negative. (top) GDS II drawing of the negative 2-2 pattern at a narrower FOV. (bottom) SEM image of Pattern 2-2 at a comparable HFW. 

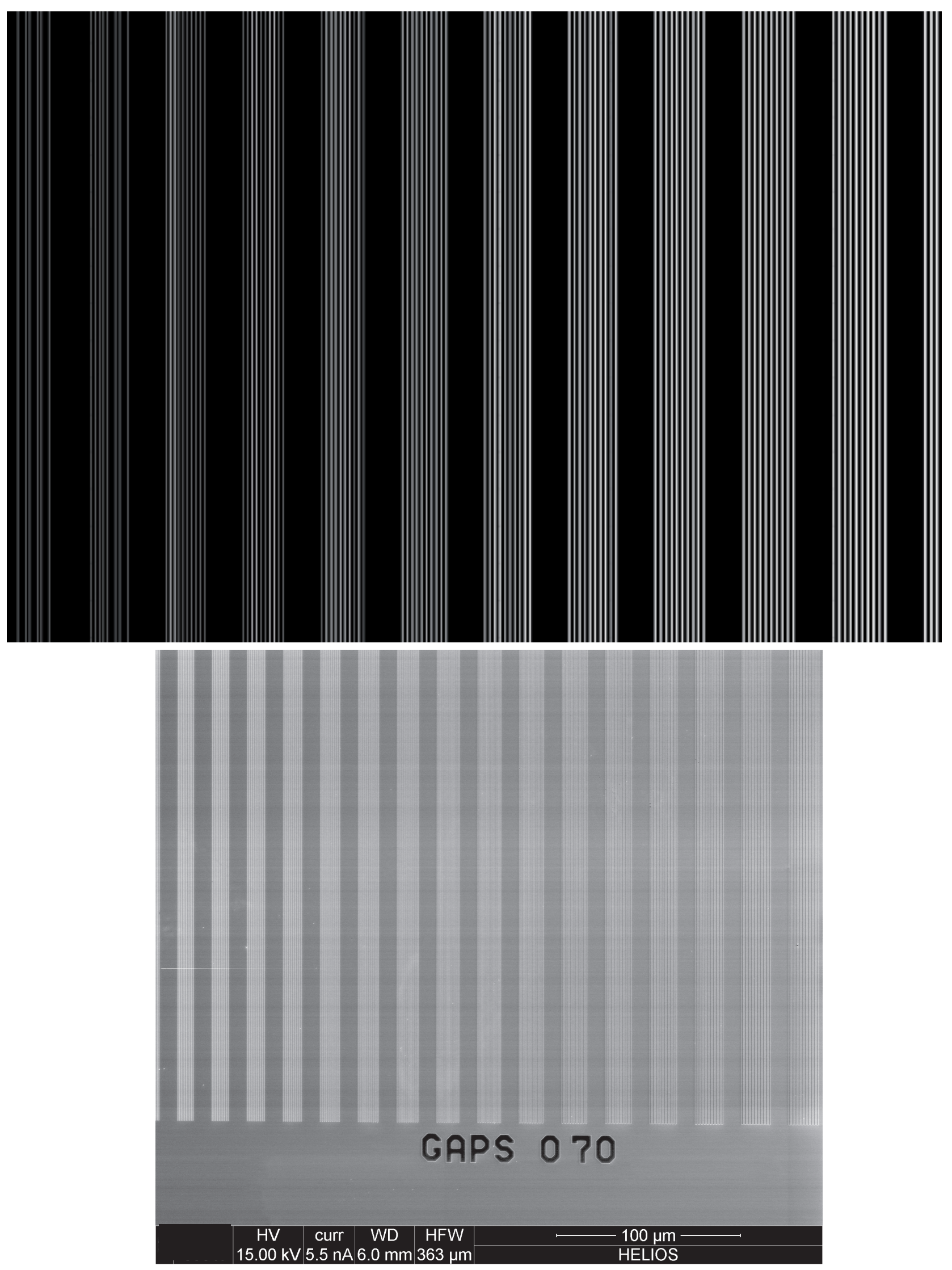

Plate 31 - Pattern 2-2 - Multiple Gap Lines Negative 
Plate 32 - Patterns 2-3, 2-4, 2-5 and 2-6 - Multiple Size Square Structures Section. (top) Wide FOV of GDS II drawing of Patterns 2-3, 2-4 and 2-5. Pattern 2-6 edge is at the extreme right part of the FOV. (bottom) GDS II drawing of Pattern 2-3 and a portion of Pattern 2-4 at reduced FOV. Pattern 2-3 has nominally $10 \mu \mathrm{m}$ square structures and Pattern 2-4 has nominally $5 \mu \mathrm{m}$ square structures. 

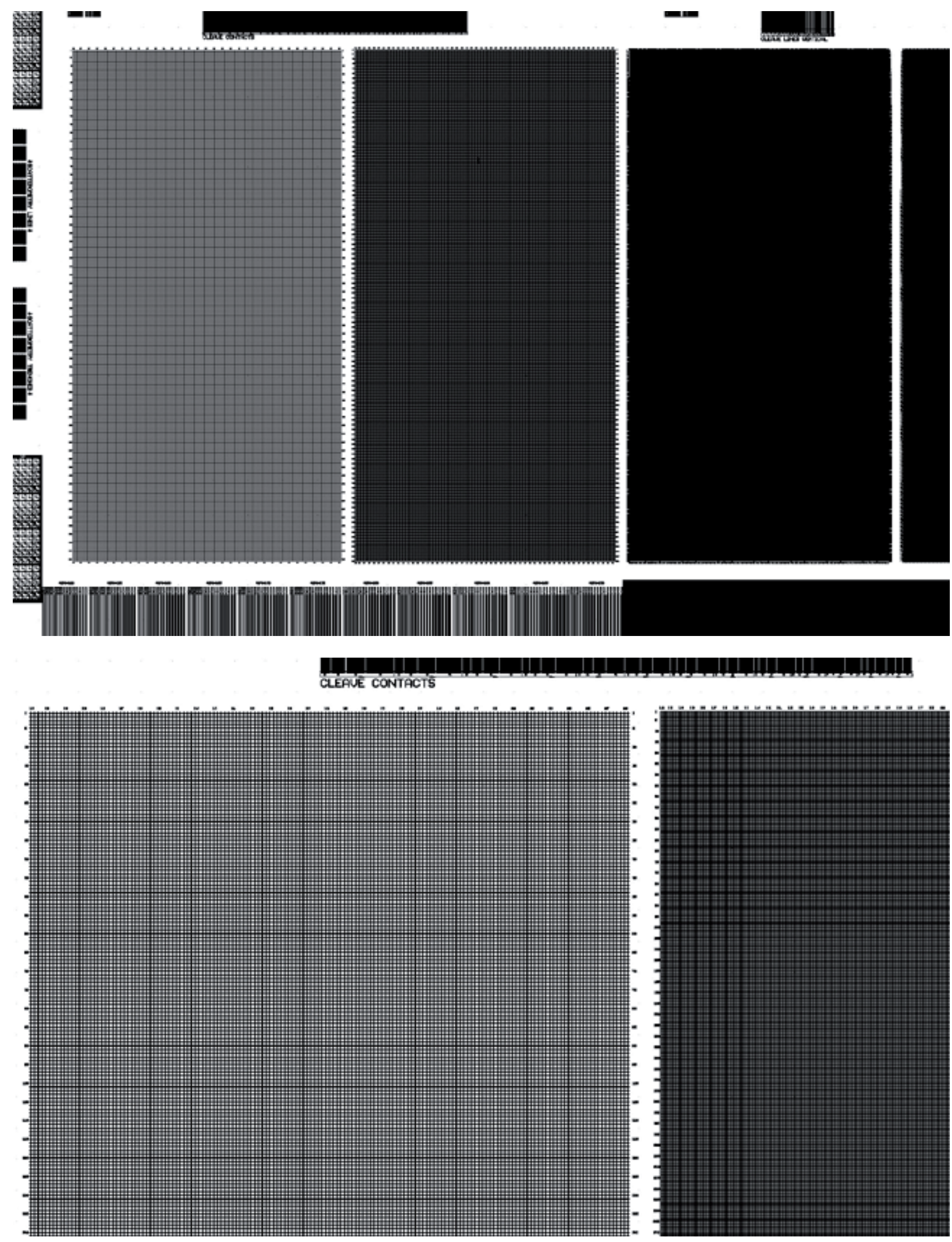

Plate 32 - Patterns 2-3, 2-4, and 2-5. and 2-6 - Multiple Size Square Structures Section 
Plate 33 - Pattern 2-3 - Multiple Size Square Structures Section. (top) Reduced FOV view of GDS II drawing of Pattern 2-3 showing the nominal $10 \mu \mathrm{m}$ structures. (bottom) SEM micrograph of Pattern 2-3. 

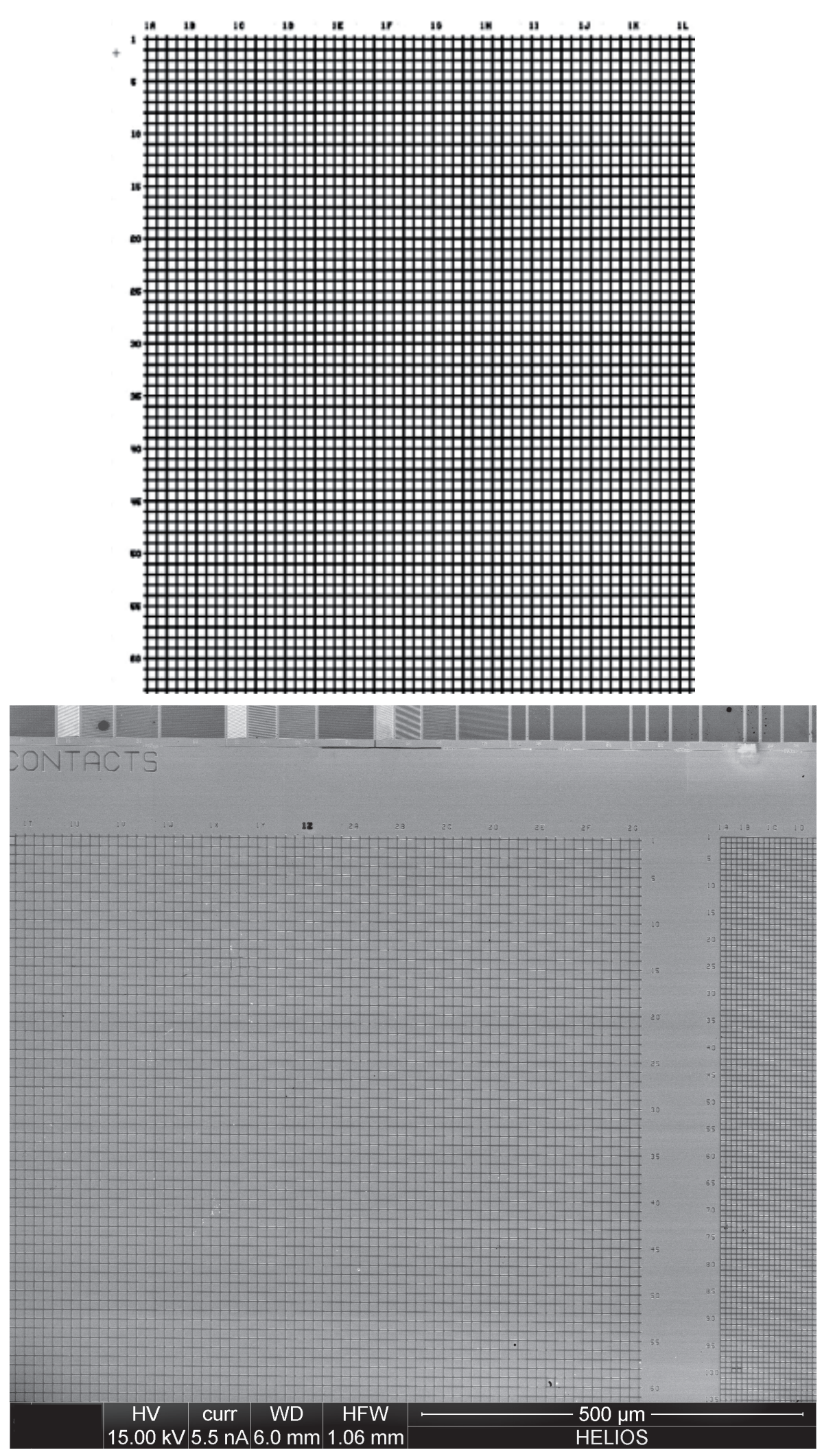

Plate 33 - Patterns 2-3 - Multiple Size Square Structures Section 
Plate 34 - Pattern 2-3 - Multiple Size Square Structures Section. (top, bottom) SEM Micrographs of Pattern 2-3 showing the nominal $10 \mu \mathrm{m}$ structures. 

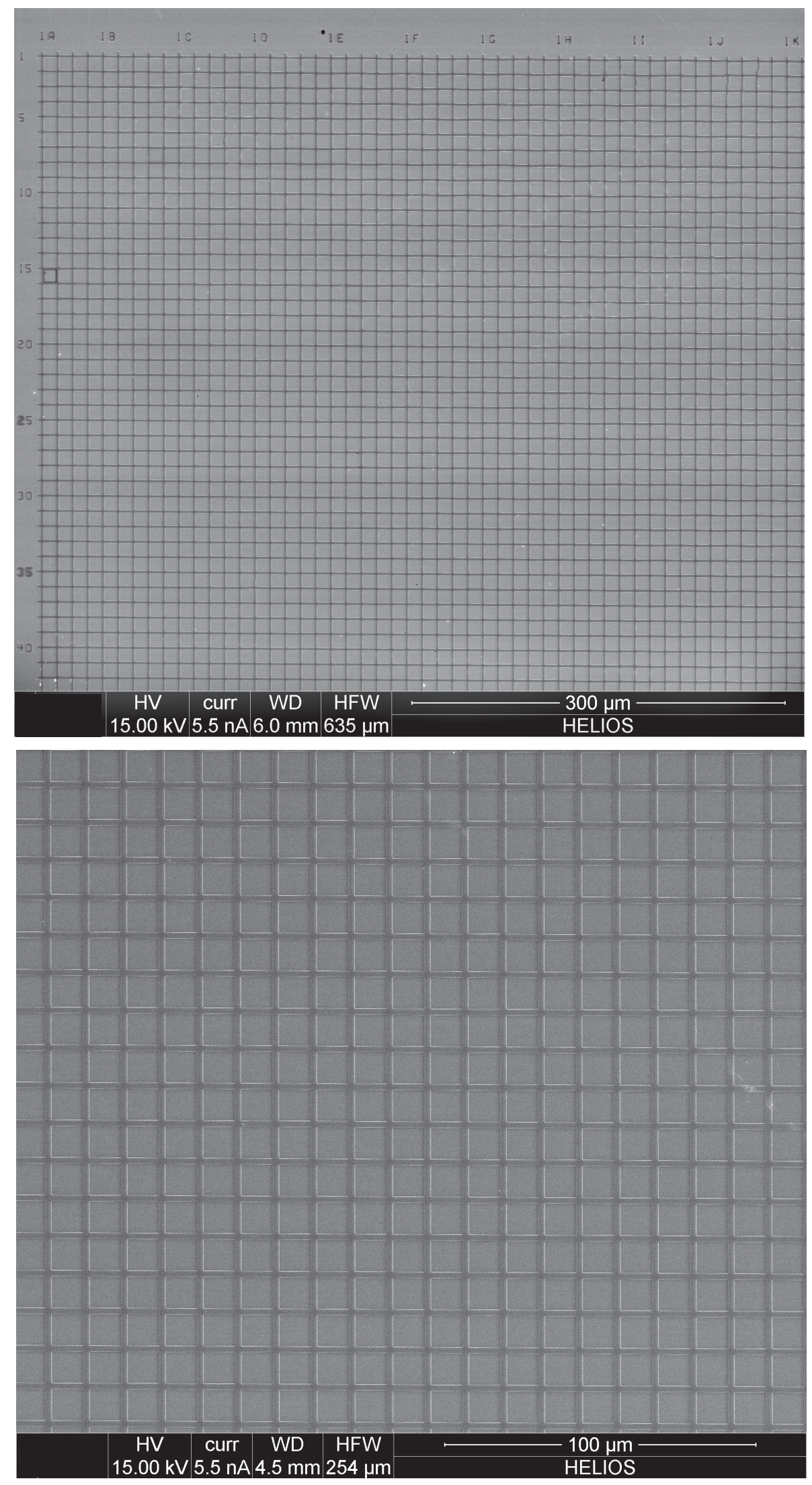

Plate 34 - Pattern - 2-3 - Multiple Size Square Structures Section 
Plate 35 - Pattern 2-4, Multiple Size Square Structures Section. (top) Reduced FOV view of GDS II drawing of Pattern 2-4 showing the nominal $5 \mu \mathrm{m}$ structures. (bottom) SEM micrograph of Pattern 2-4. 

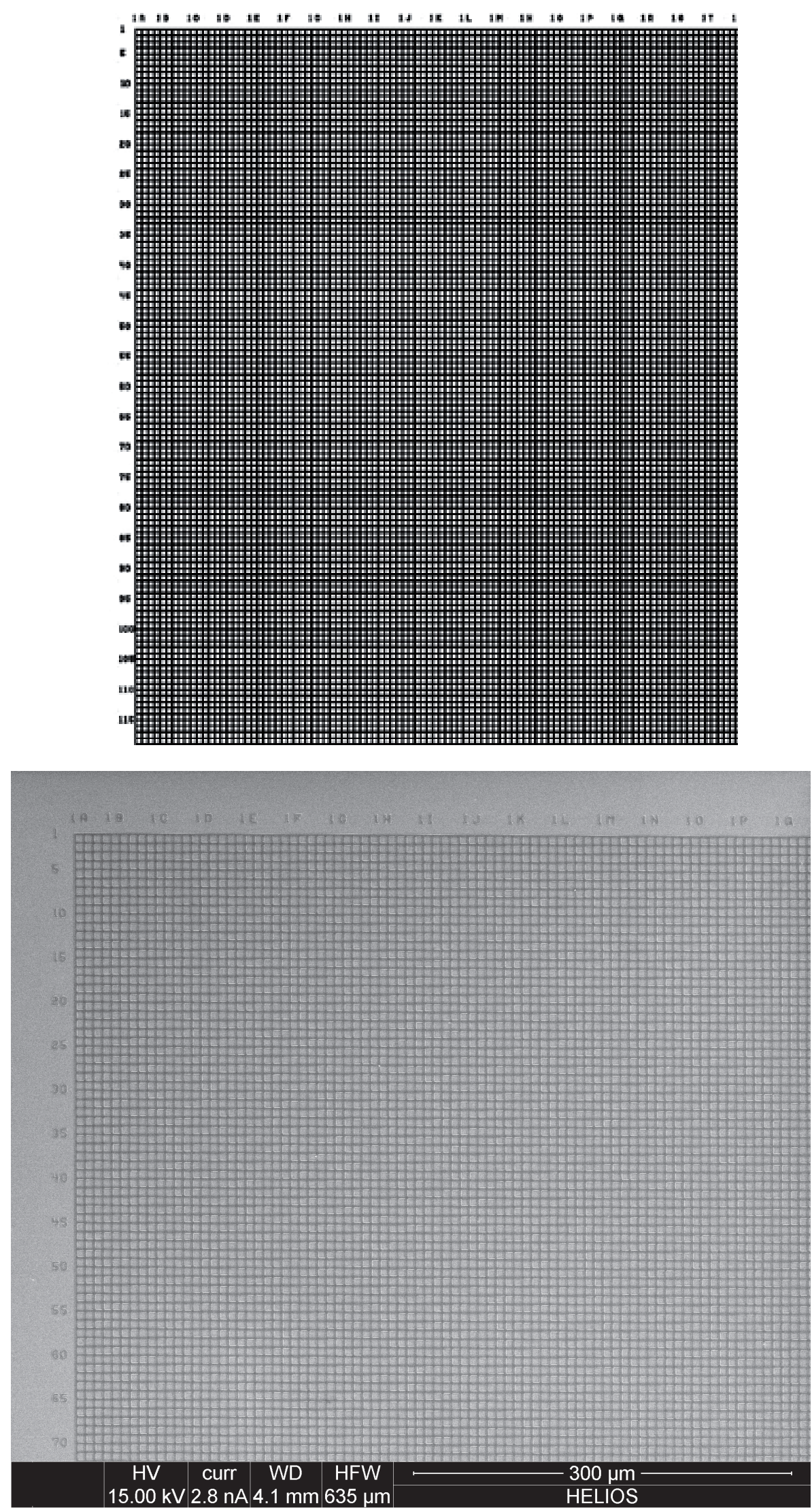

Plate 35 - Pattern 2-4 - Multiple Size Square Structures Section 
Plate 36 - Pattern 2-4, Multiple Size Square Structures Section. (top, bottom) SEM micrographs of Pattern 2-4 showing the nominal $5 \mu \mathrm{m}$ structures. 

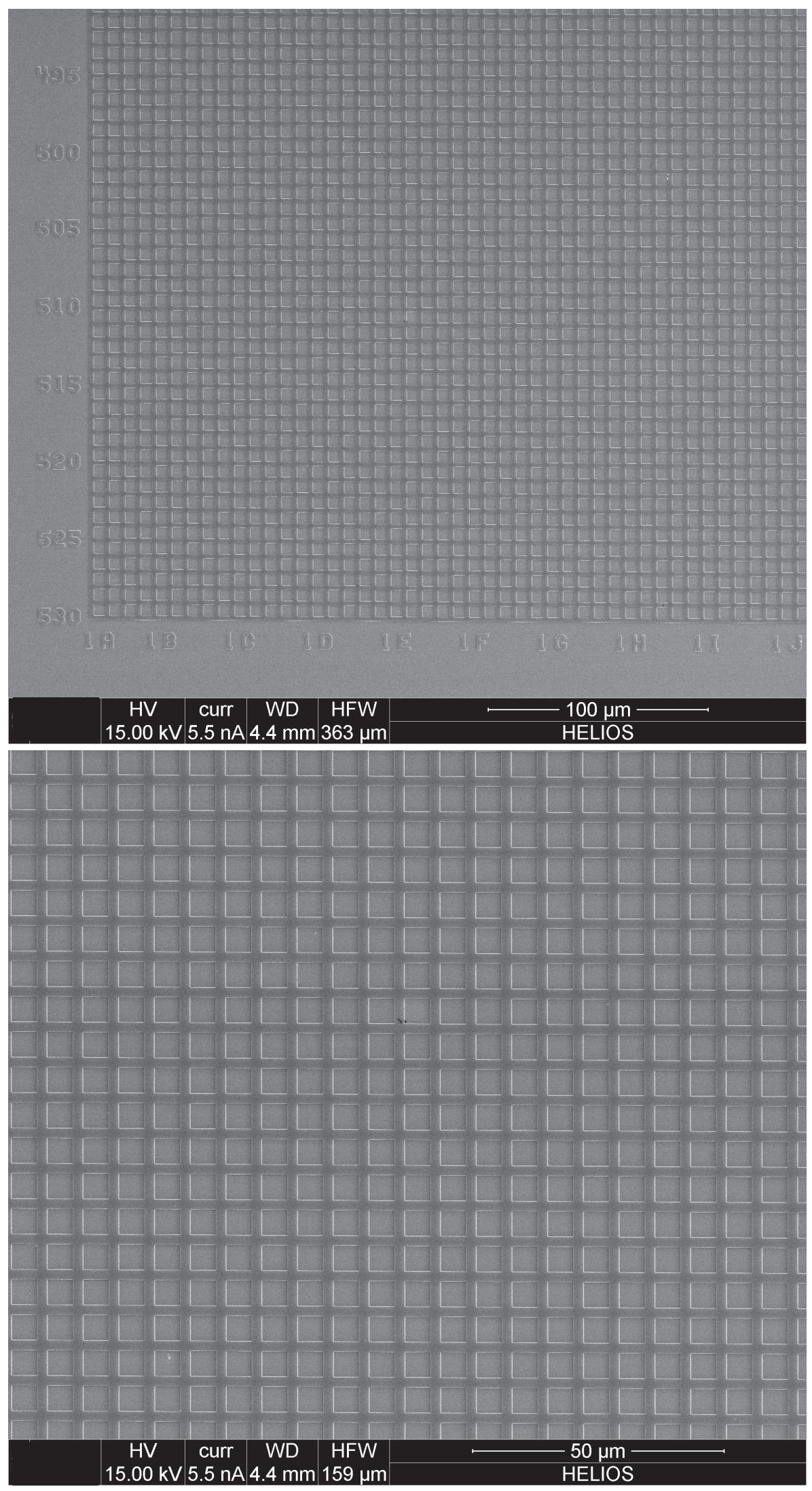
Plate 37 - Pattern 2-5, Multiple Size Square Structures Section. (top) Reduced FOV view of GDS II drawing of Pattern 2-5 showing the nominal $1 \mu \mathrm{m}$ structures. (bottom) SEM micrograph of Pattern $2-5$. 


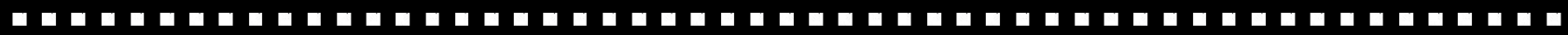

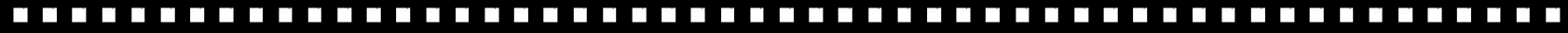

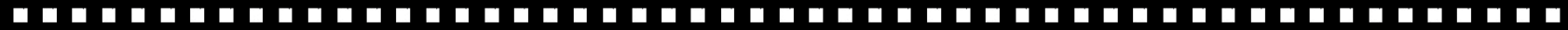
|

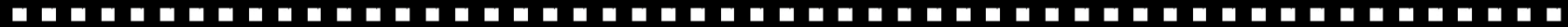

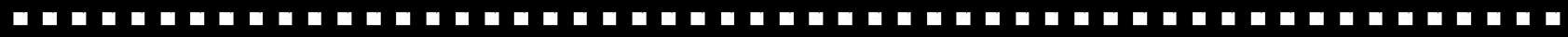

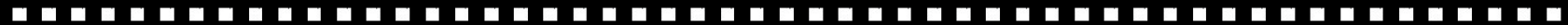

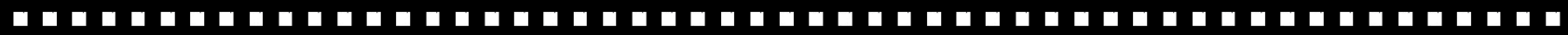

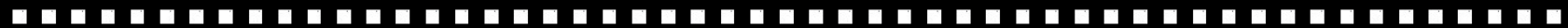

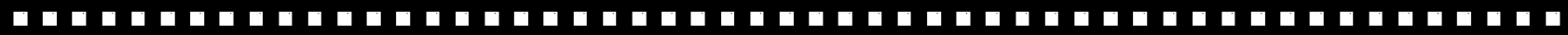

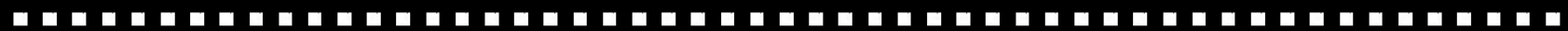

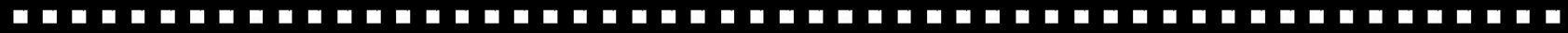

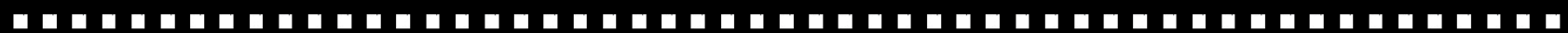

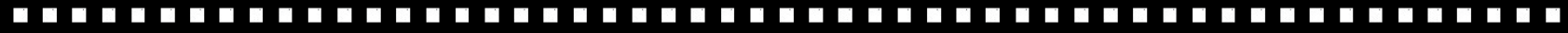
(10)

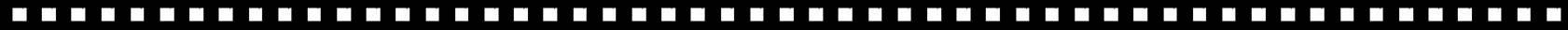

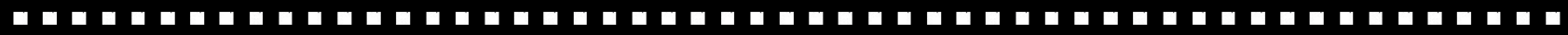

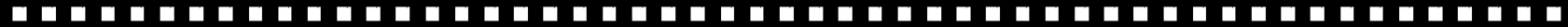

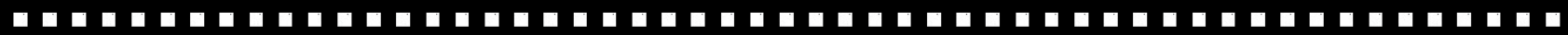

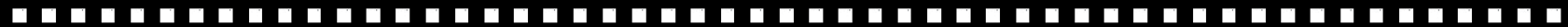
|

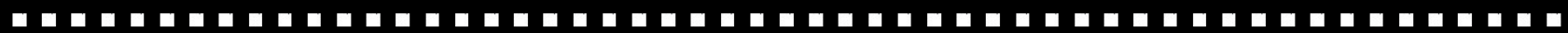

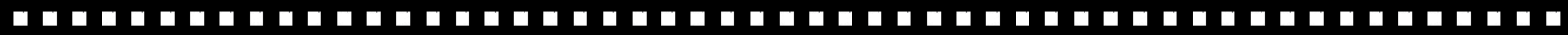

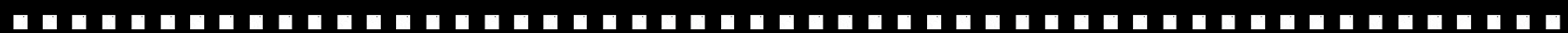

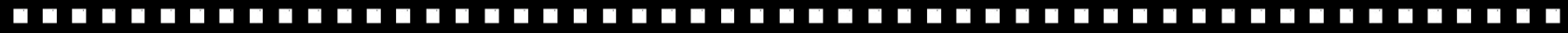

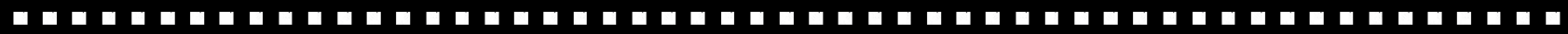

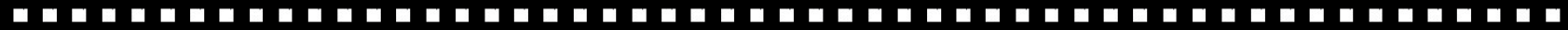

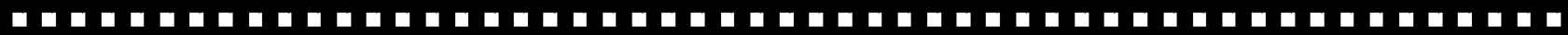

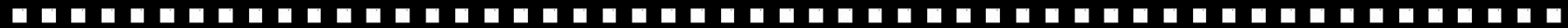

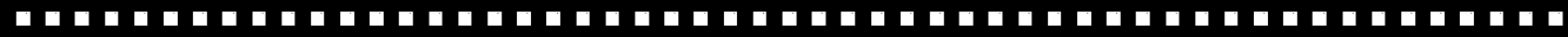

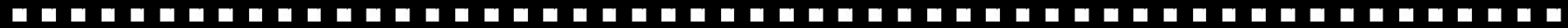

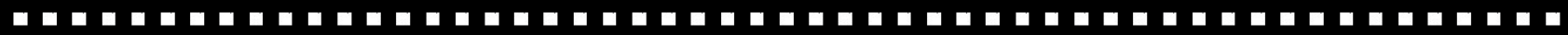

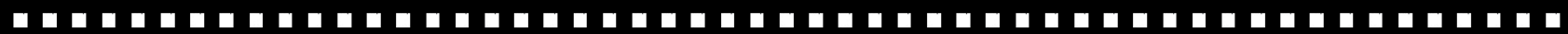

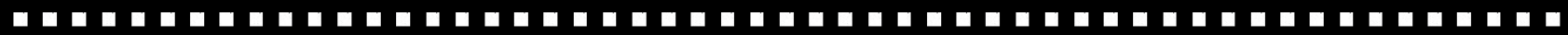

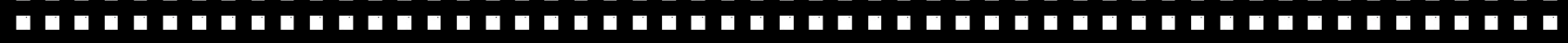

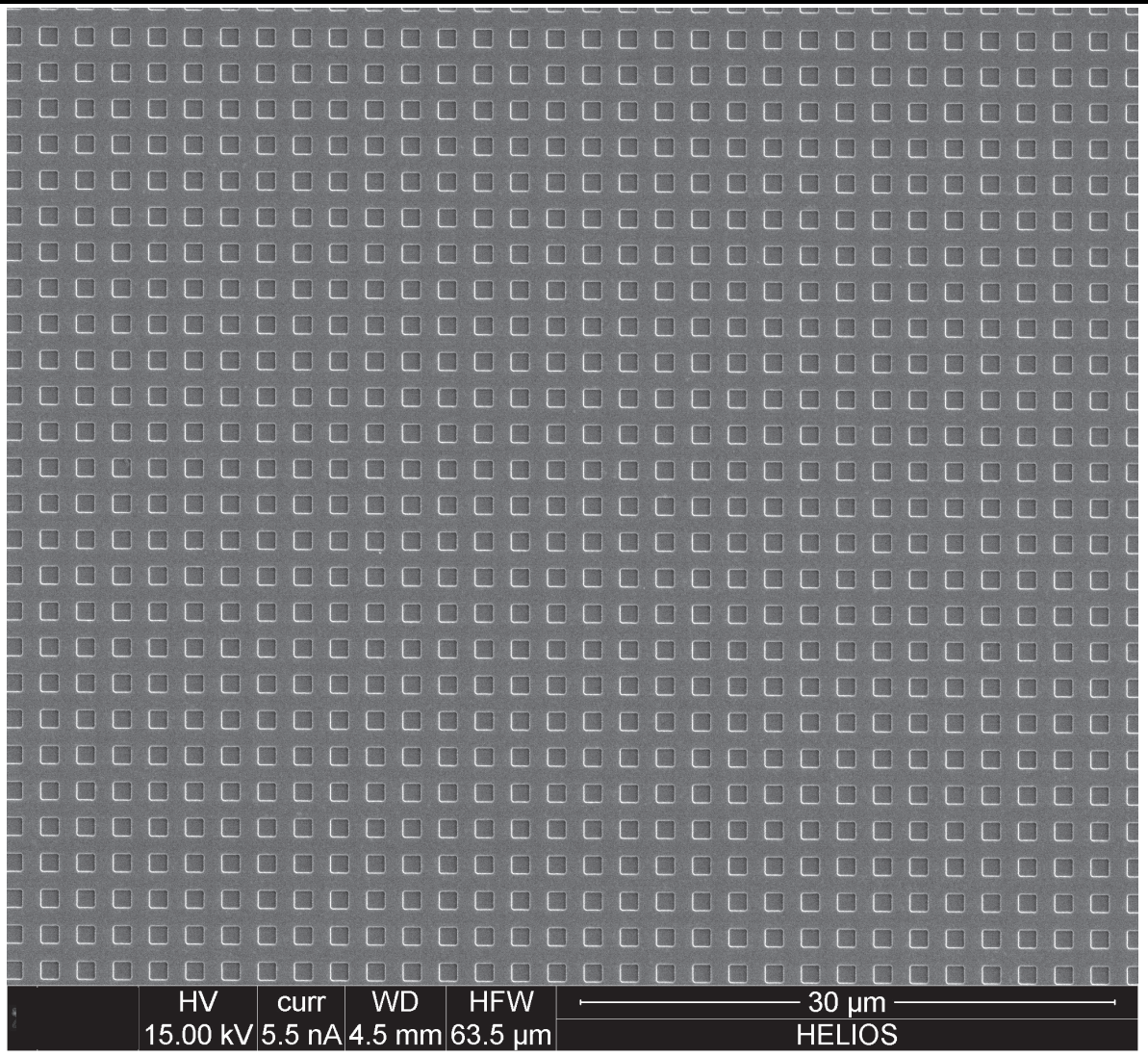

Plate 38 - Pattern 2-5 - Multiple Size Square Structures Section 
Plate 38 - Pattern 2-6 - Multiple Size Square Structures Section. (top) Reduced FOV view of GDS II drawing of Pattern 2-6 showing the nominal $0.5 \mu \mathrm{m}$. (bottom) SEM micrograph of Pattern 2-6. 

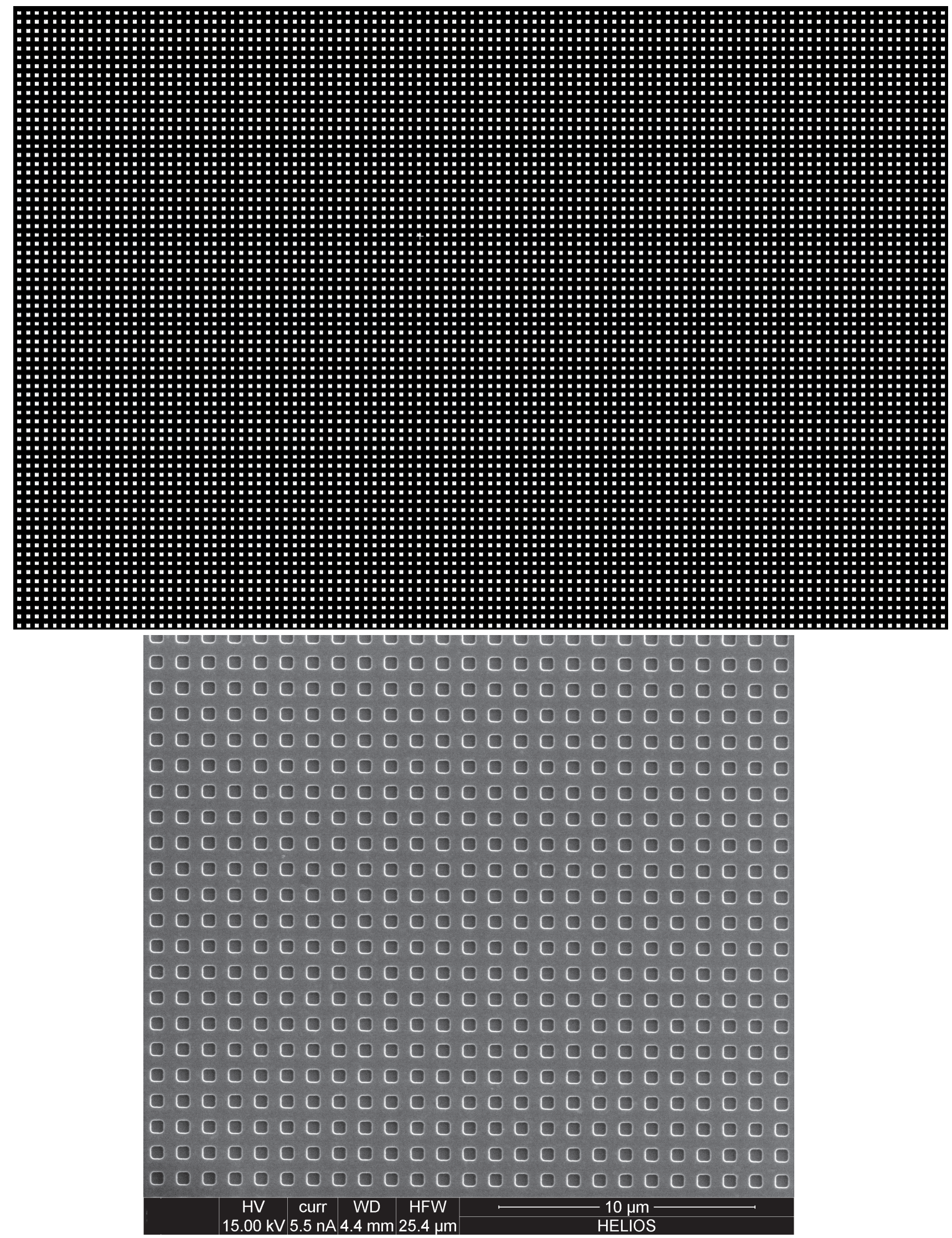

Plate 38 - Pattern 2-6 - Multiple Size Square Structures Section 
Page intentionally left blank 
SECTION 3 
Plate 39. Section 3-0 Overall. (top) GDS II line drawing of the entire RM 8820 pattern highlighting Section 3 with the three additional major sub-patterns numerically identified. (bottom) GDS II detail drawing of Section 3 showing the three additional major sub-patterns. Pattern 3-1 the Global Alignment Test Pattern is large enough to be included in the wide FOV. Pattern 3-1 is the global alignment test pattern (described below).

\section{Explanation:}

Global Alignment Test Patterns. This module is an array of optical global alignment patterns. There are $180 \mu \mathrm{m}$ dense line symbols and solid polysilicon symbols. There are 5 different cells within the Optical GAP module. The cells are based on the following symbol sizes: $45 \mu \mathrm{m}, 90 \mu \mathrm{m}, 180 \mu \mathrm{m}, 270$ $\mu \mathrm{m}, 540 \mu \mathrm{m}$. In this module the dense line symbols appear black. 

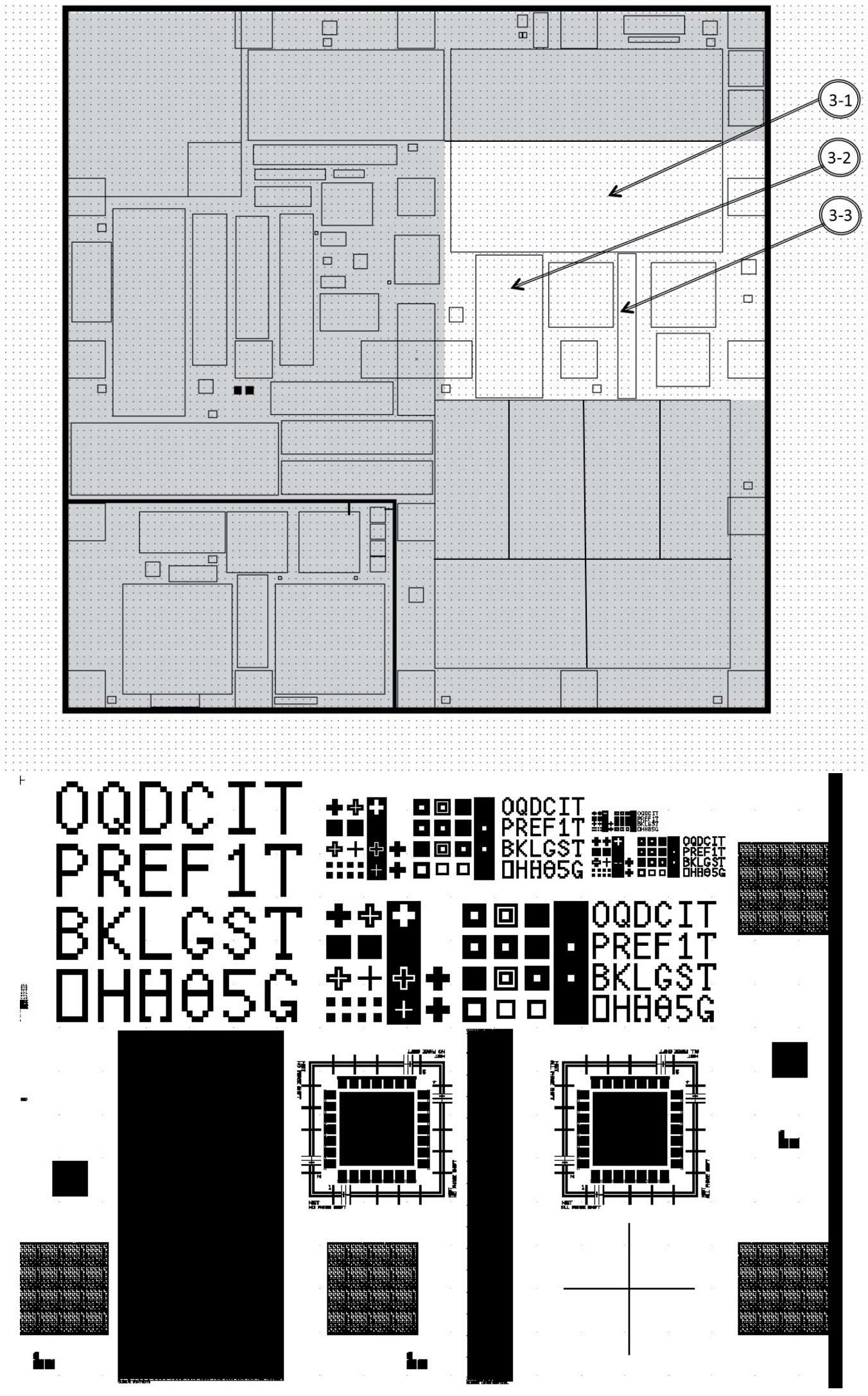

Plate 39 - Section 3 - Overall and Pattern 3-1 
Plate 40 - Pattern 3-1 Global Alignment Test Pattern. (top, bottom) SEM Micrographs of a portion of the Global Alignment Test Pattern section. 

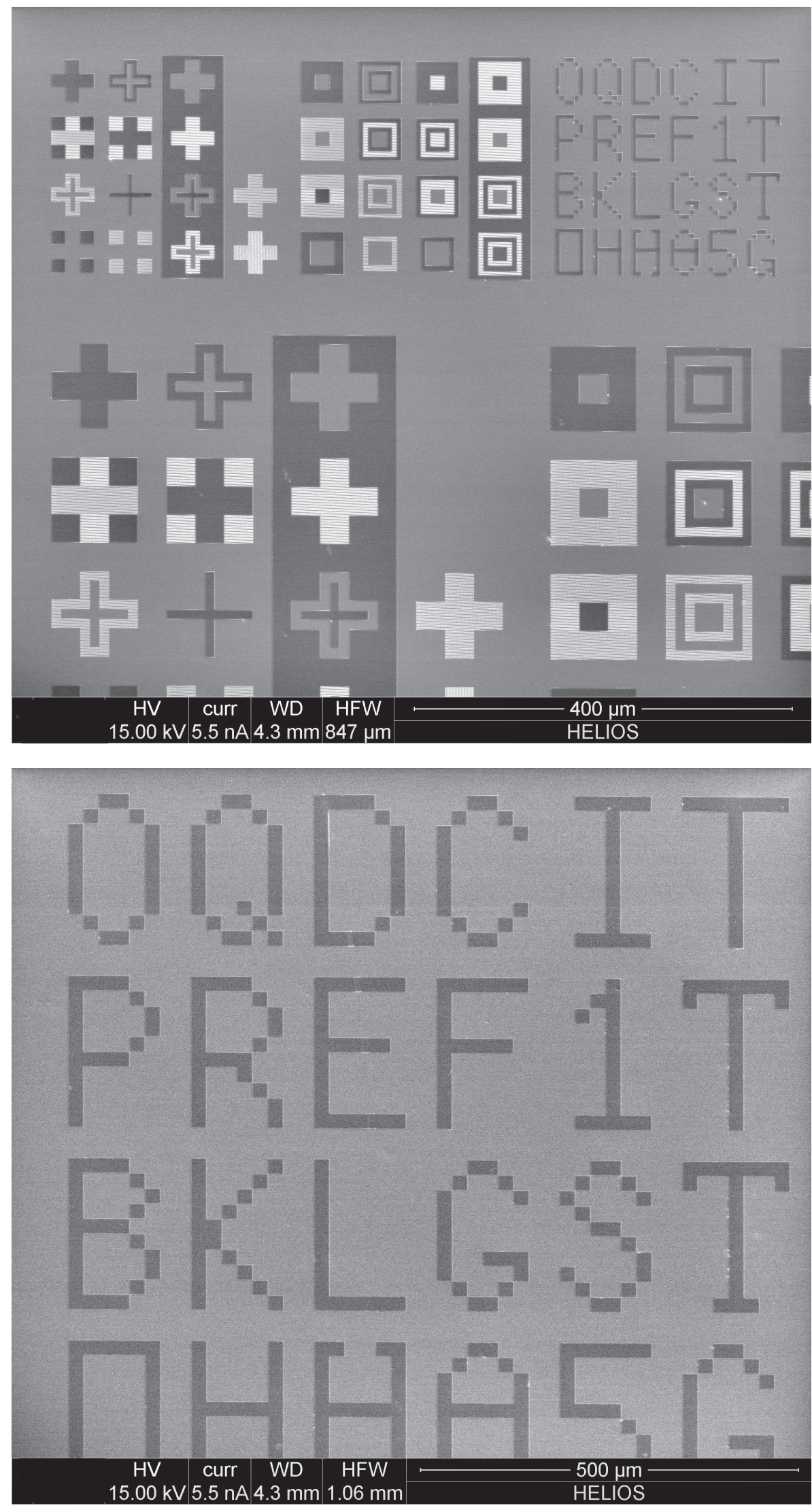

Plate 40 - Pattern 3-1 - Global Alignment Test Pattern. 
Plate 41 - Pattern 3-2 - Cleave lines. (top) GDS II drawing of the cleave lines pattern at wide FOV. The large gaps delimiting the different size and ratio of the lines are apparent in the image. (bottom) SEM micrograph of the cleave lines at a low FOV. The length of these lines is nominally $4 \mathrm{~mm}$.

\section{Explanation:}

Line/space Cleave. This large set of patterns and large area in size was designed for general line-space measurements and process control purposes and specifically for cleaving for cross-sectional SEM. With their $4 \mathrm{~mm}$ long line length, these structures are easily cleavable making it feasible to make topdown and crosssectional measurements at the same locations. Various pattern recognition marks are also included to allow for navigation of any tool under test and of the cross-section SEM cleave line if using a micro-cleaver. All the main nominal CD sizes in other marks are represented, with several different pitch ratios and also an isolated line and isolated space of these nominal dimensions. This module includes both horizontal and vertical versions. 

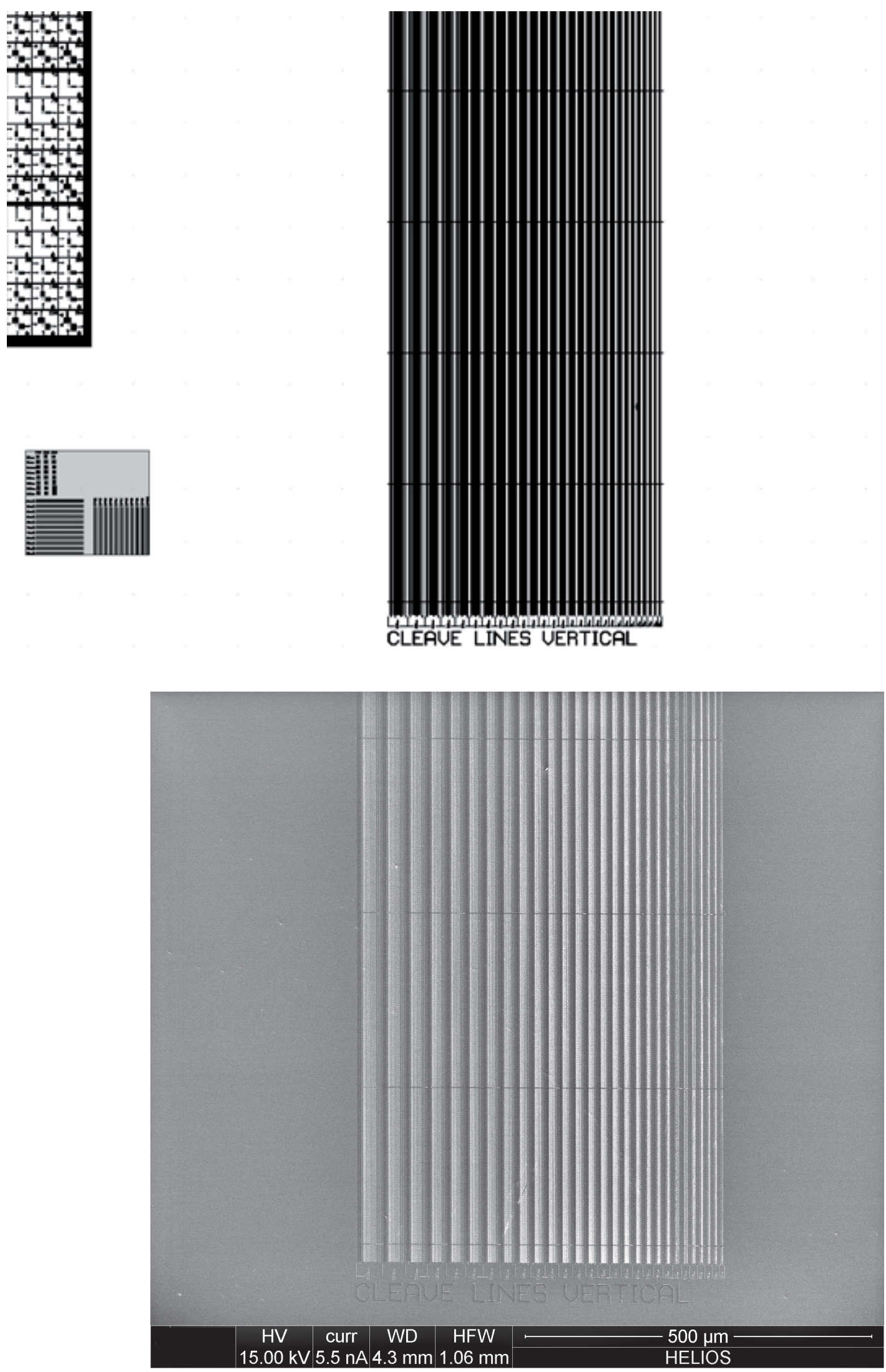

Plate 41 - Pattern 3-2 - Cleave lines 
Plate 42. Pattern 3-2 - Cleave lines. (top) GDS II drawing of the cleave lines pattern at smaller FOV. The large gaps delimiting the different size and ratio of the lines are apparent in the image. (bottom) SEM micrograph of the cleave lines at a small FOV. The length of these lines is nominally $4 \mathrm{~mm}$. 

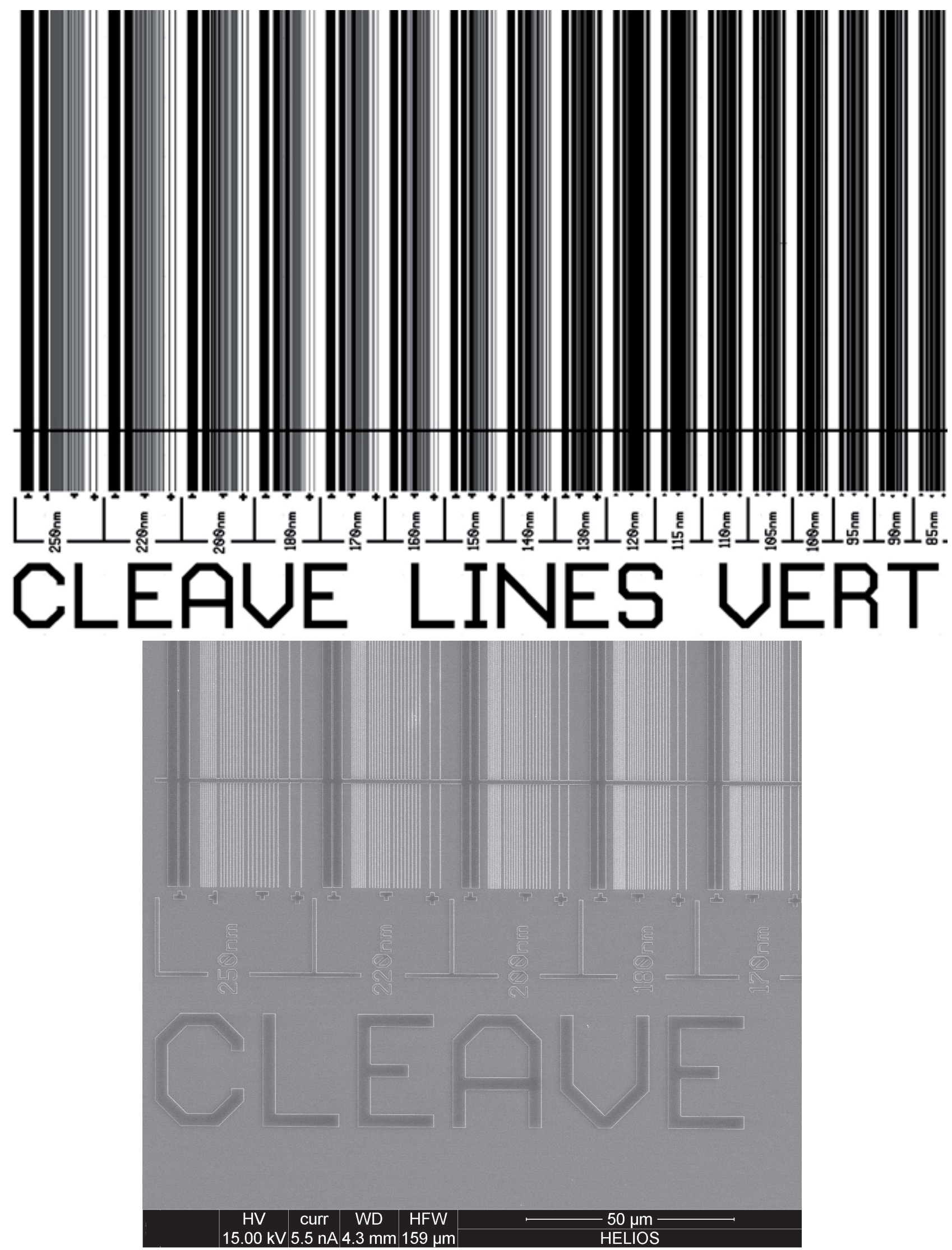

Plate 42 - Pattern 3-2.- Cleave lines. 
Plate 43 - Pattern 3-3 - Cleave contacts. (top) GDS II drawing of the cleave contacts pattern at wide FOV. The large gaps delimiting the different size and ratio of the contact holes are apparent in the image. (bottom) SEM micrograph of Pattern 3-3 at wide FOV. The length of these arrays is nominally 4 $\mathrm{mm}$. 

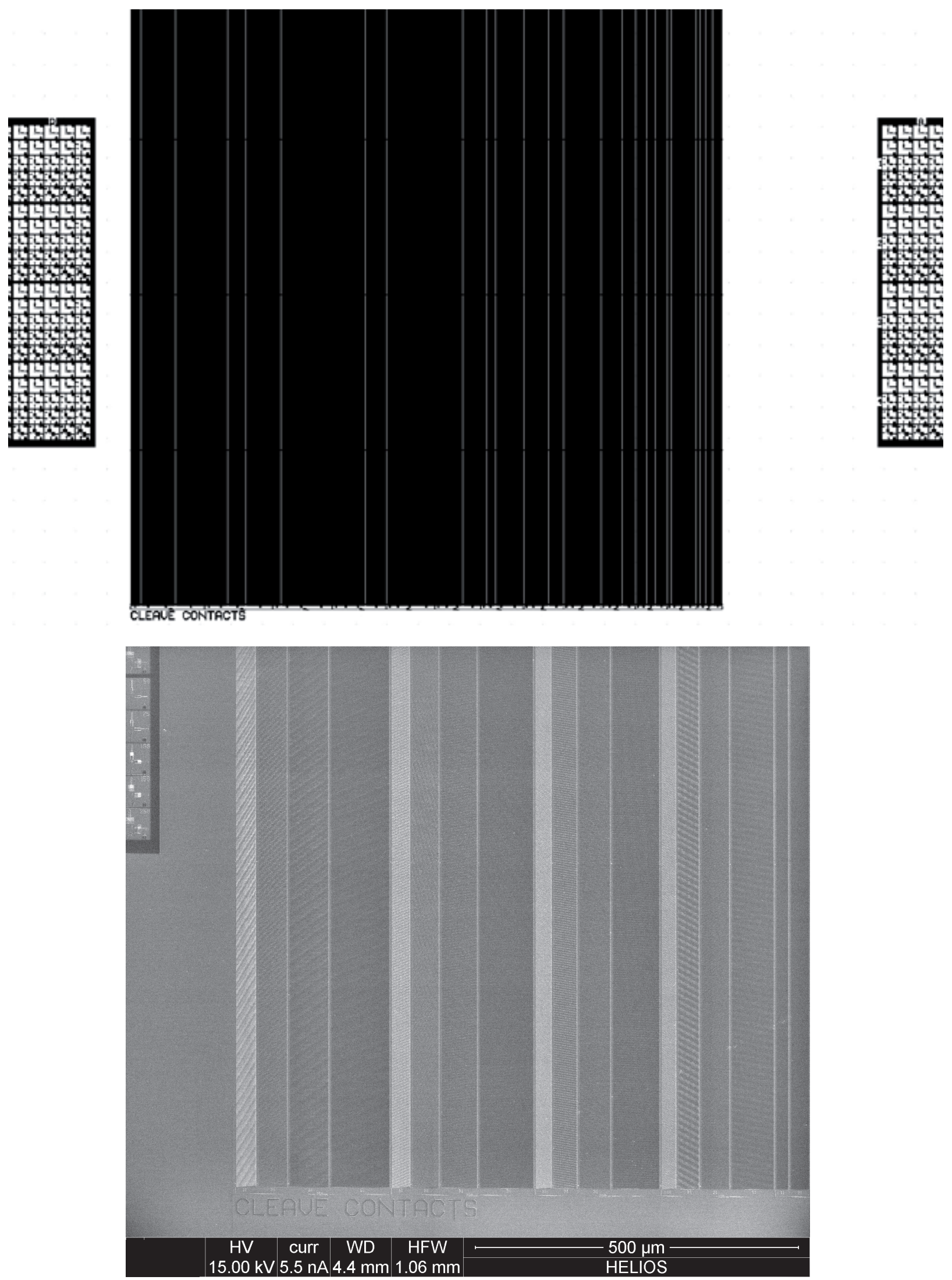

Plate 43 - Pattern 3-3 - Cleave contacts. 
Plate 44 - Patterns 3-3 - Cleave contacts. Reduced FOV view of GDS II drawing of Pattern 3-3 showing the nominal $250 \mathrm{~nm}$ contact hole structures with a nominal 1:1 spacing ratio. (bottom, left) SEM micrograph of a similar area. (bottom, right ) SEM micrographs at a reduced FOV. 

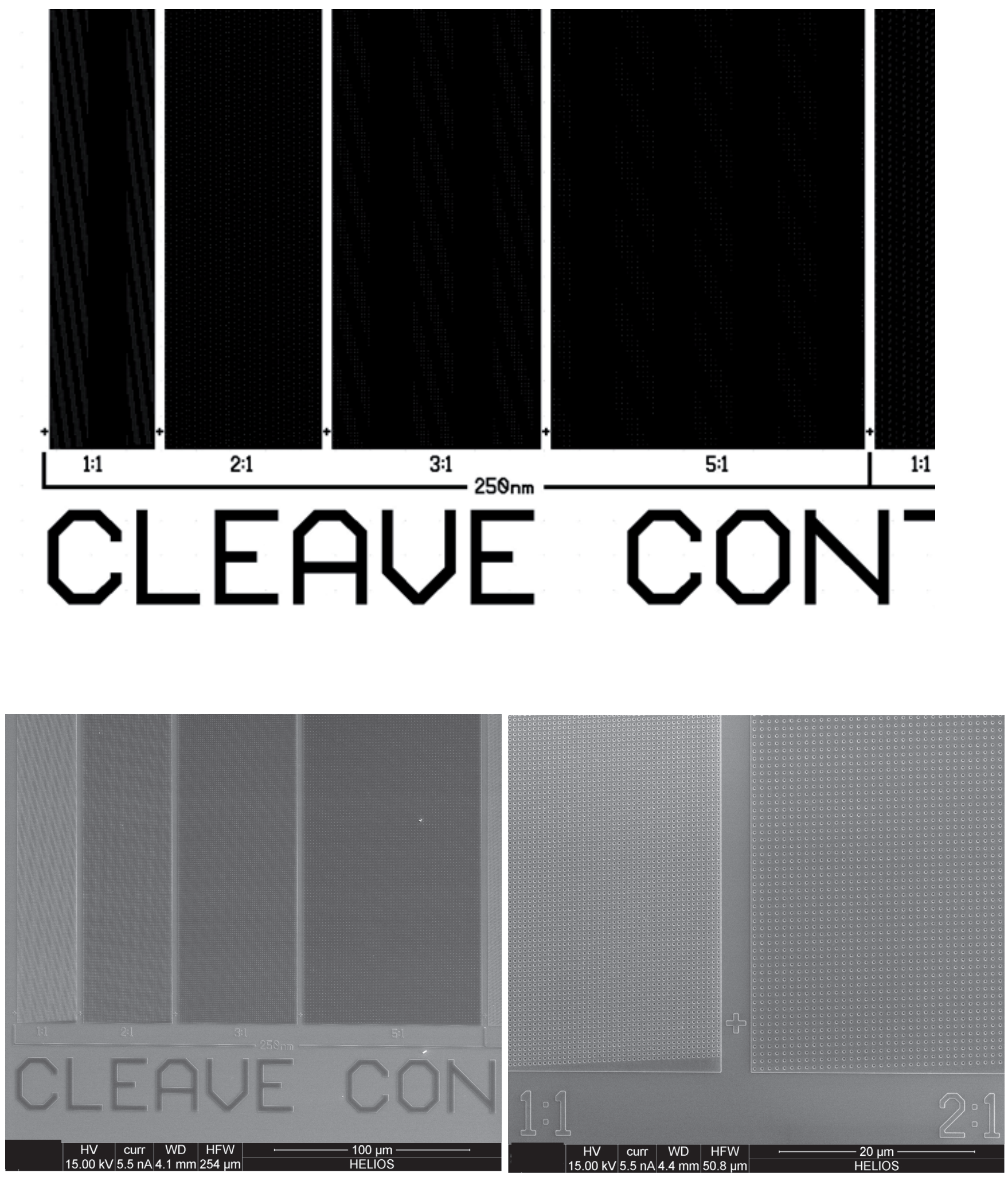
Plate 45 - Patterns 3-3 - Cleave contacts. (top) Reduced FOV view of GDS II drawing of Pattern 3-3 showing the nominal $250 \mathrm{~nm}$ contact hole structures with a nominal 1:1 spacing ratio. (bottom) SEM micrograph of a similar area. 

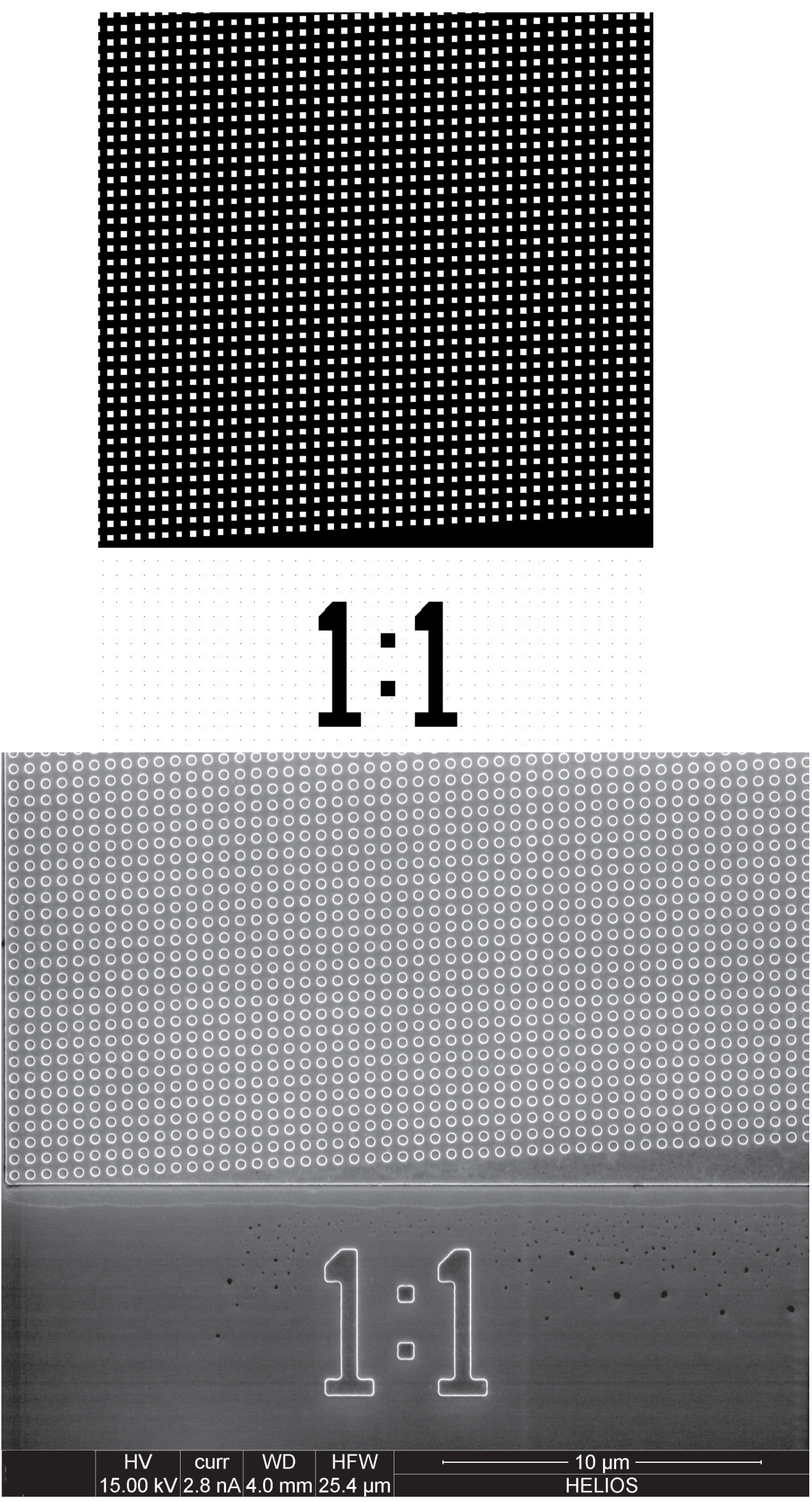

Plate 45 - Pattern 3-2 - Cleave contacts 
Plate 46 - Patterns 3-3 - Cleave contacts. Reduced FOV view of GDS II drawing of Pattern 3-3 showing the nominal $0.25 \mu \mathrm{m}$ contact hole structures with a nominal 2:1 spacing ratio. (bottom,) SEM micrograph of a similar area. 

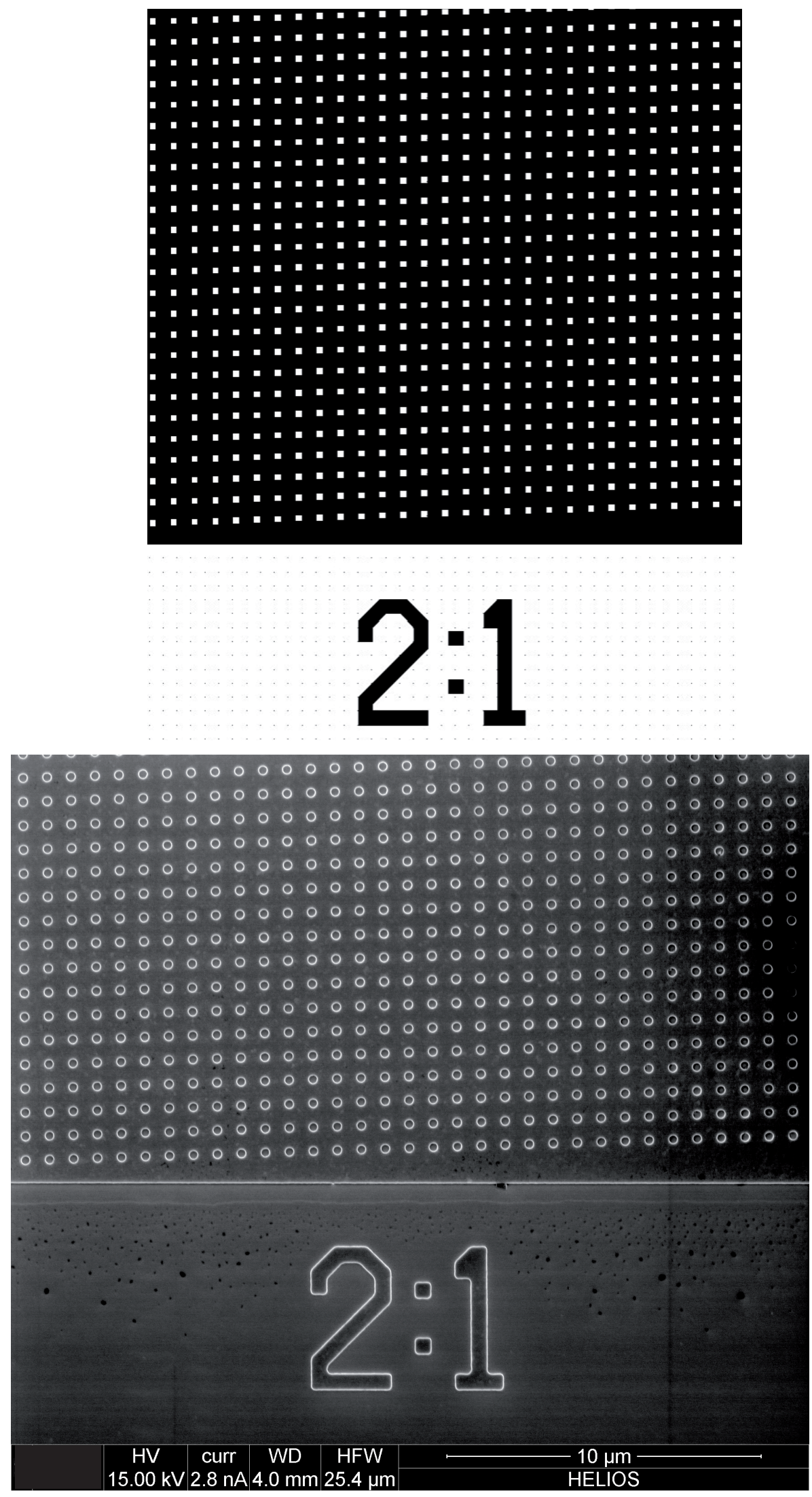

Plate 46 - Pattern 3-2 - Cleave contacts 
Plate 47 - Patterns 3-3 - Cleave contacts. (top) Reduced FOV view of GDS II drawing of Pattern 3-3 showing the nominal $0.25 \mu \mathrm{m}$ contact hole structures with a nominal 3:1 spacing ratio. (bottom) SEM of a similar area. 

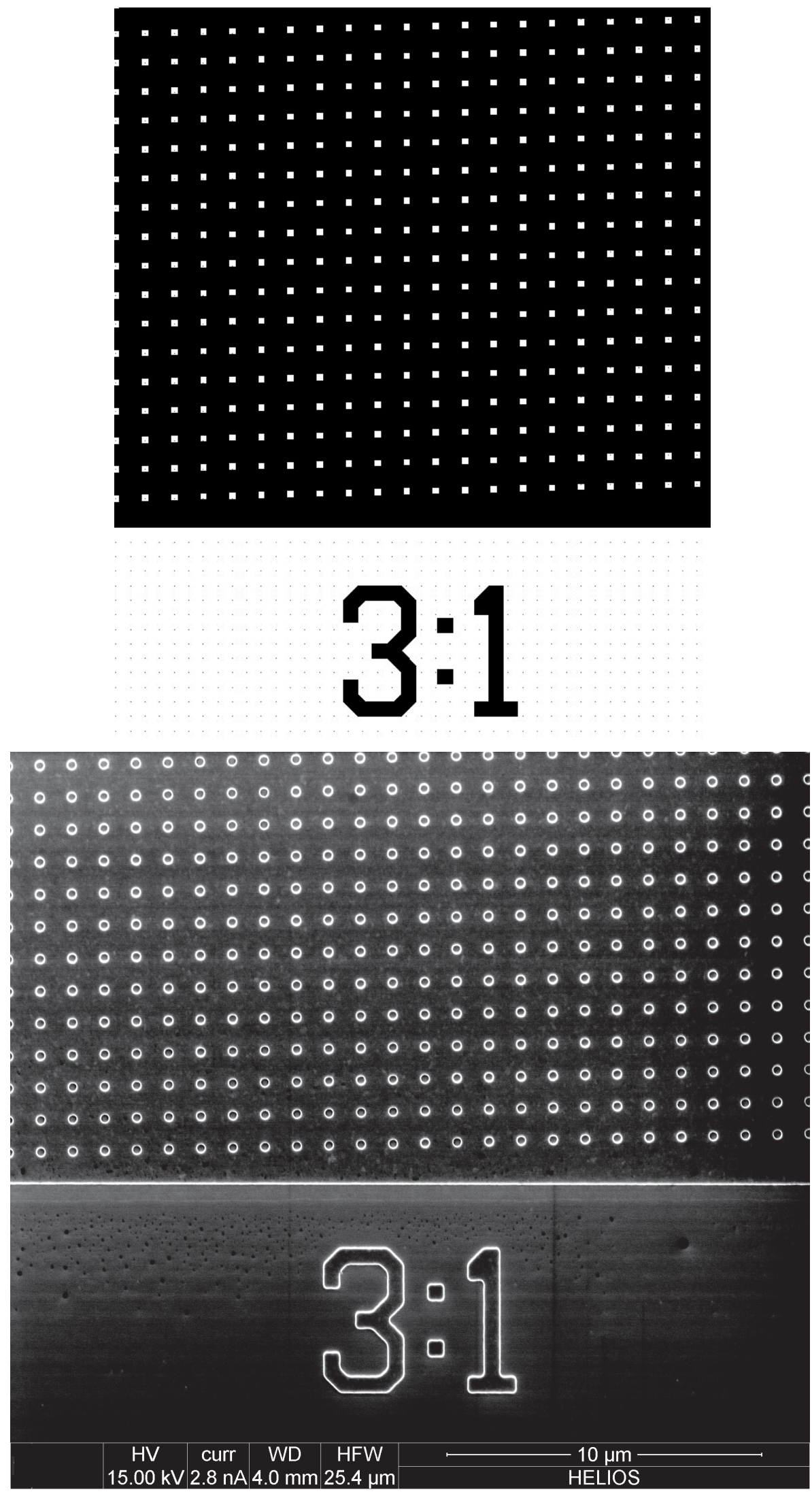

Plate 47 - Pattern 3-2 - Cleave contacts 
Plate 48 - Patterns 3-3 - Cleave contacts. (top) Reduced FOV view of GDS II drawing of Pattern 3-3 showing the nominal $0.25 \mu \mathrm{m}$ contact hole structures with a nominal 5:1 spacing ratio. (bottom) SEM of a similar area. 

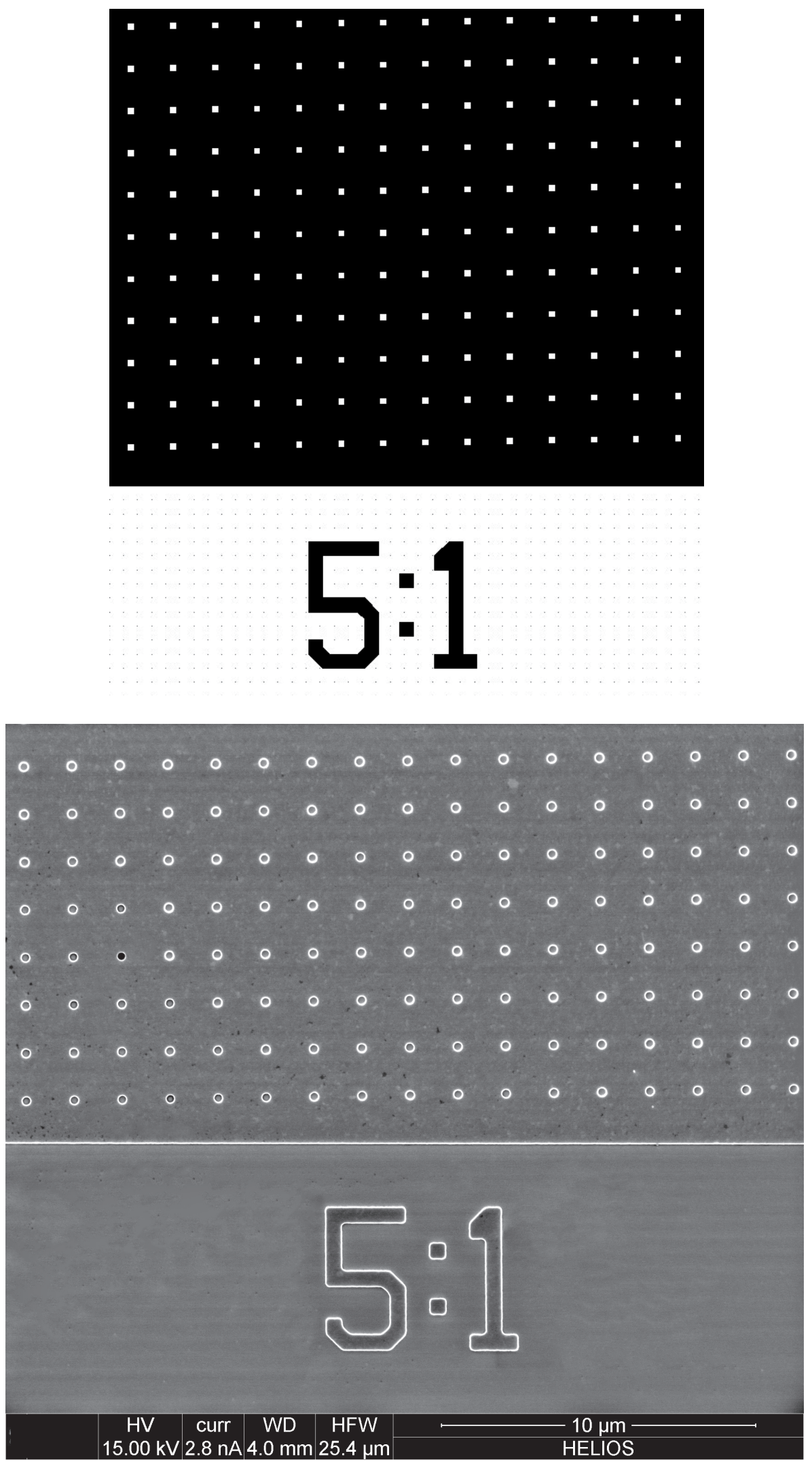

Plate 47 - Pattern 3-2 - Cleave contacts 
Page intentionally left blank 
SECTION 4 
Plate 49 - Section 4-0 overall. (top) GDS II line drawing of the entire RM 8820 pattern highlighting Section 4 with the 4 additional major sub-patterns numerically identified. (bottom) GDS II detail drawing of section 4 showing the 4 additional major sub-patterns included in this section. 

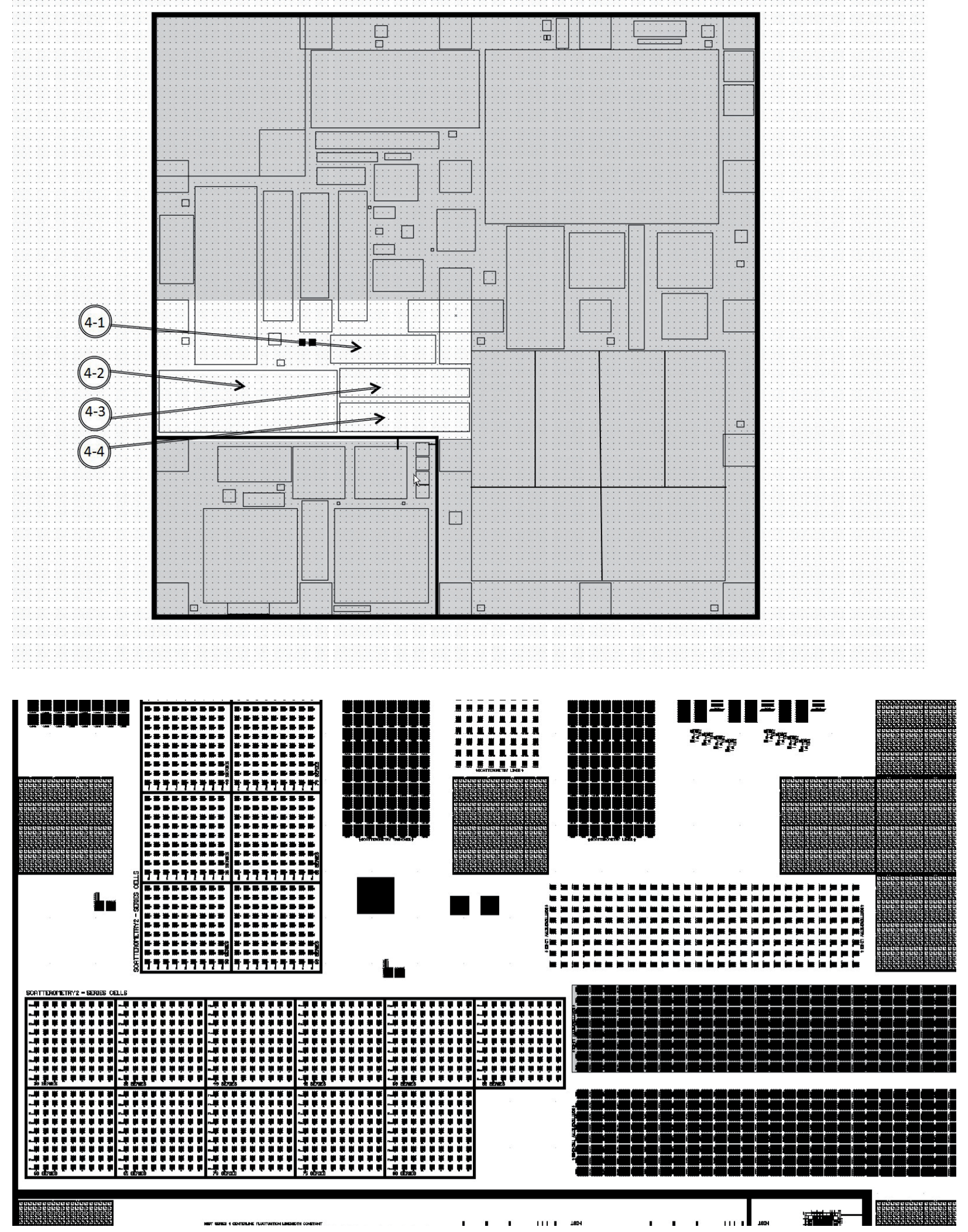

Plate 49 Section 4 - Overall 
Plate 50 - Pattern 4-1 - Scatterometry Lines. (top) GDS II drawing of the scatterometry lines Pattern 4-1 at wide FOV. (bottom) SEM micrograph of a similar area. 


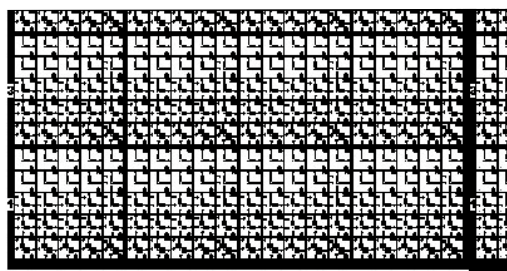

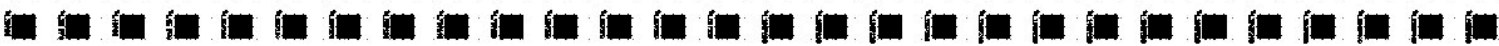

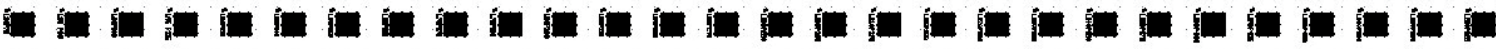

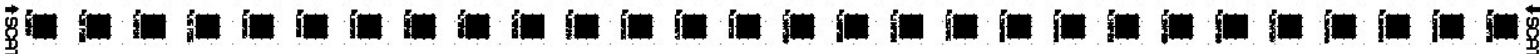

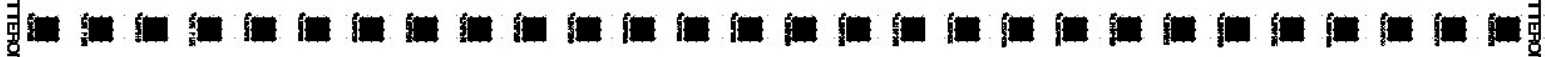

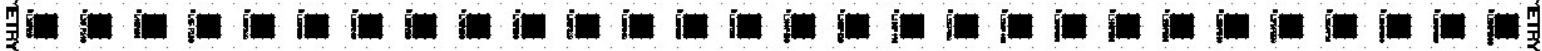

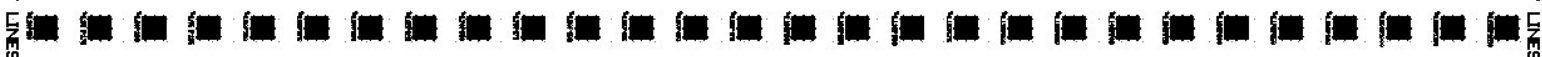

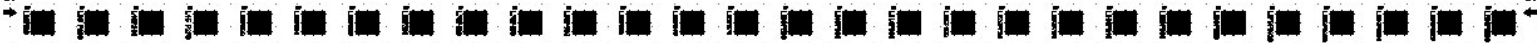

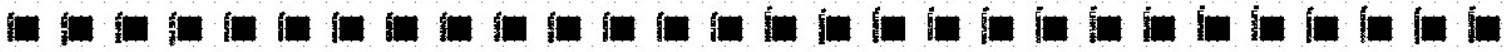
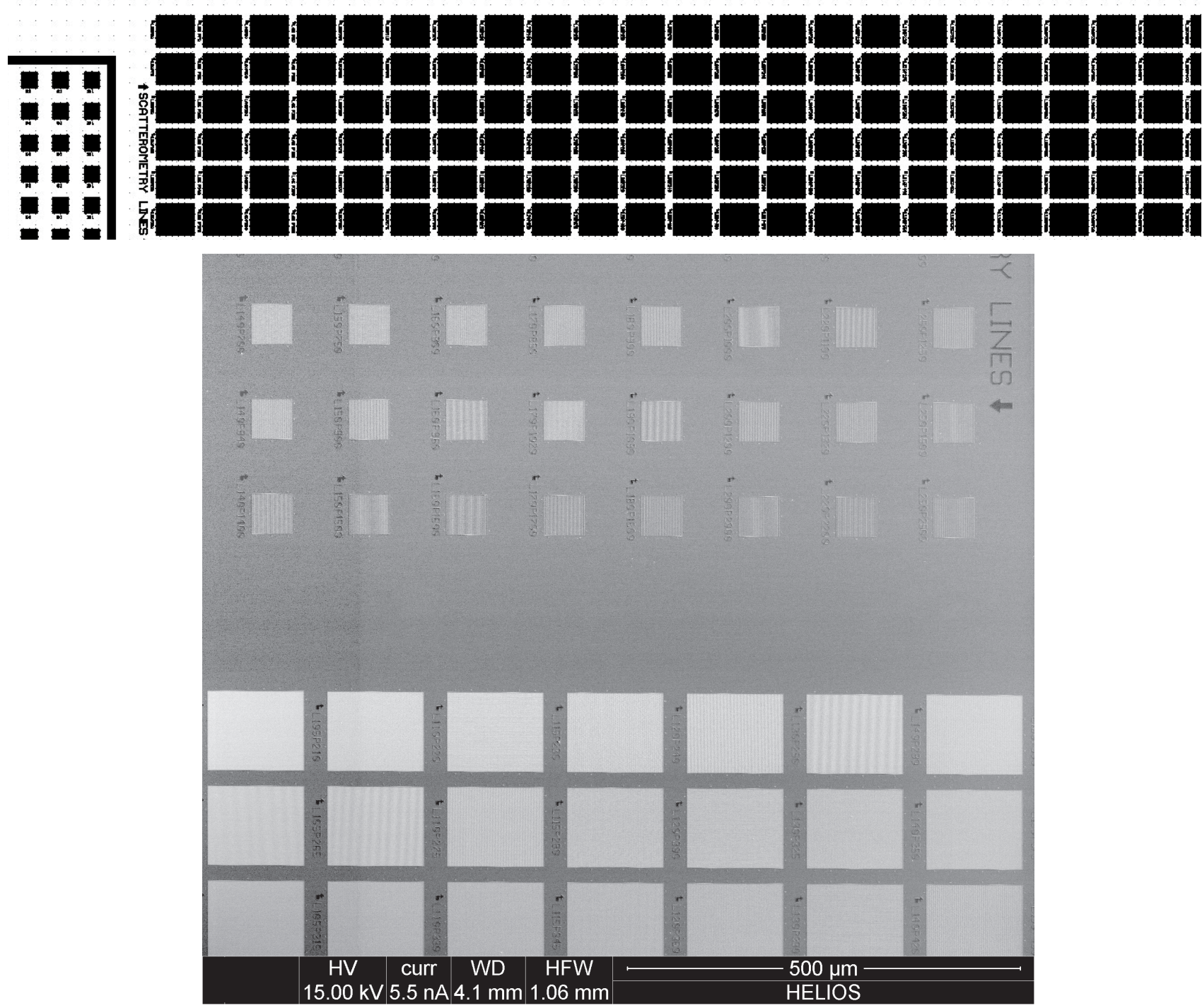

Plate 50 - Pattern 4-1 - Scatterometry Lines 
Plate 51 - Pattern 4-1 - Scatterometry Lines. (top) GDS II drawing of Pattern 4-1 at a smaller FOV showing some of the detail of the fine patterns. (bottom) SEM micrograph of a similar area. 

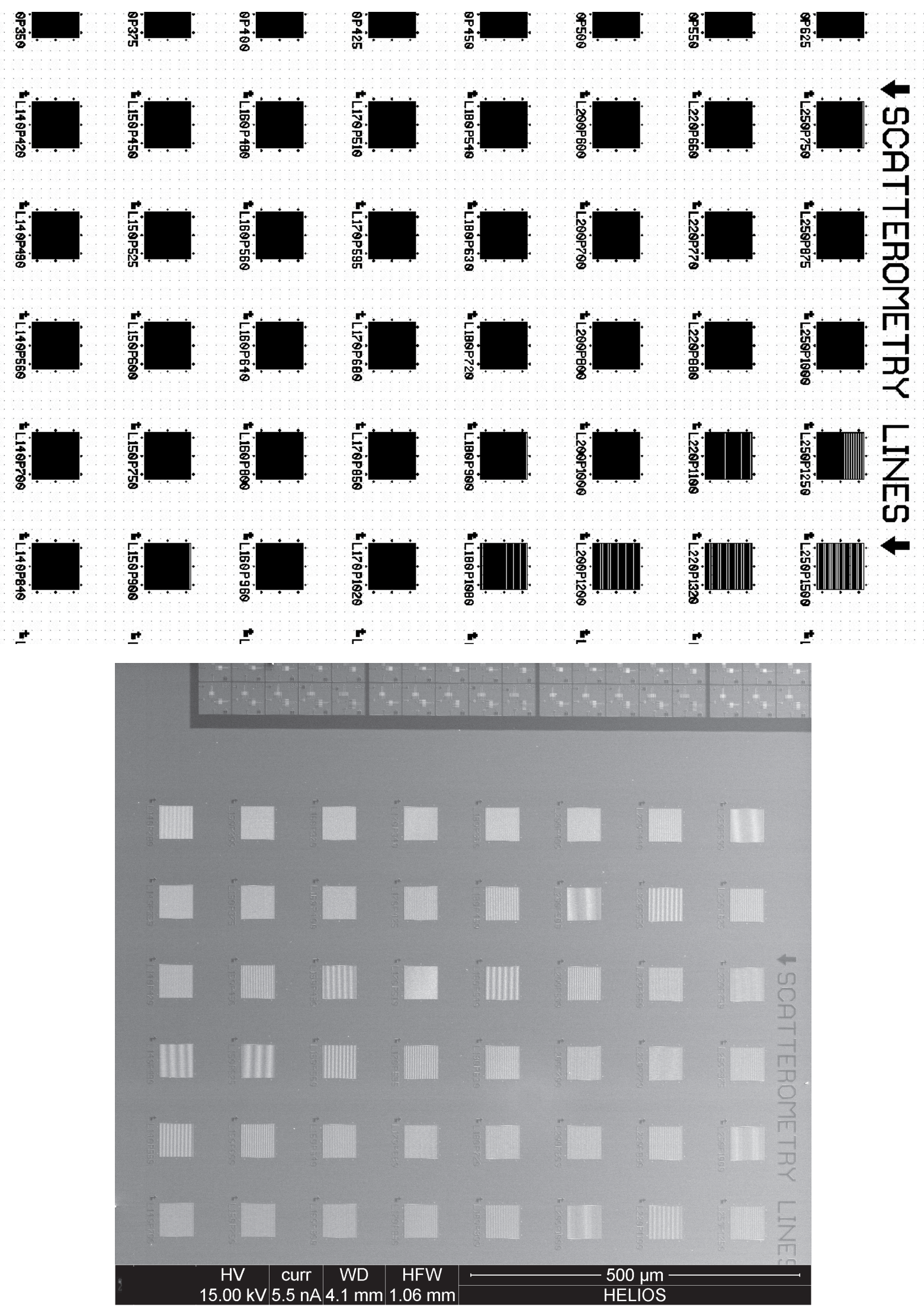

Plate 51 - Pattern 4-1 - Scatterometry Lines 
Plate 52 - Pattern 4-1 - Scatterometry Lines. (top) GDS II drawing of the scatterometry lines Pattern 4-1 at smaller FOV showing the variety of line size and line pitches available. (bottom) SEM micrograph of a similar area. 

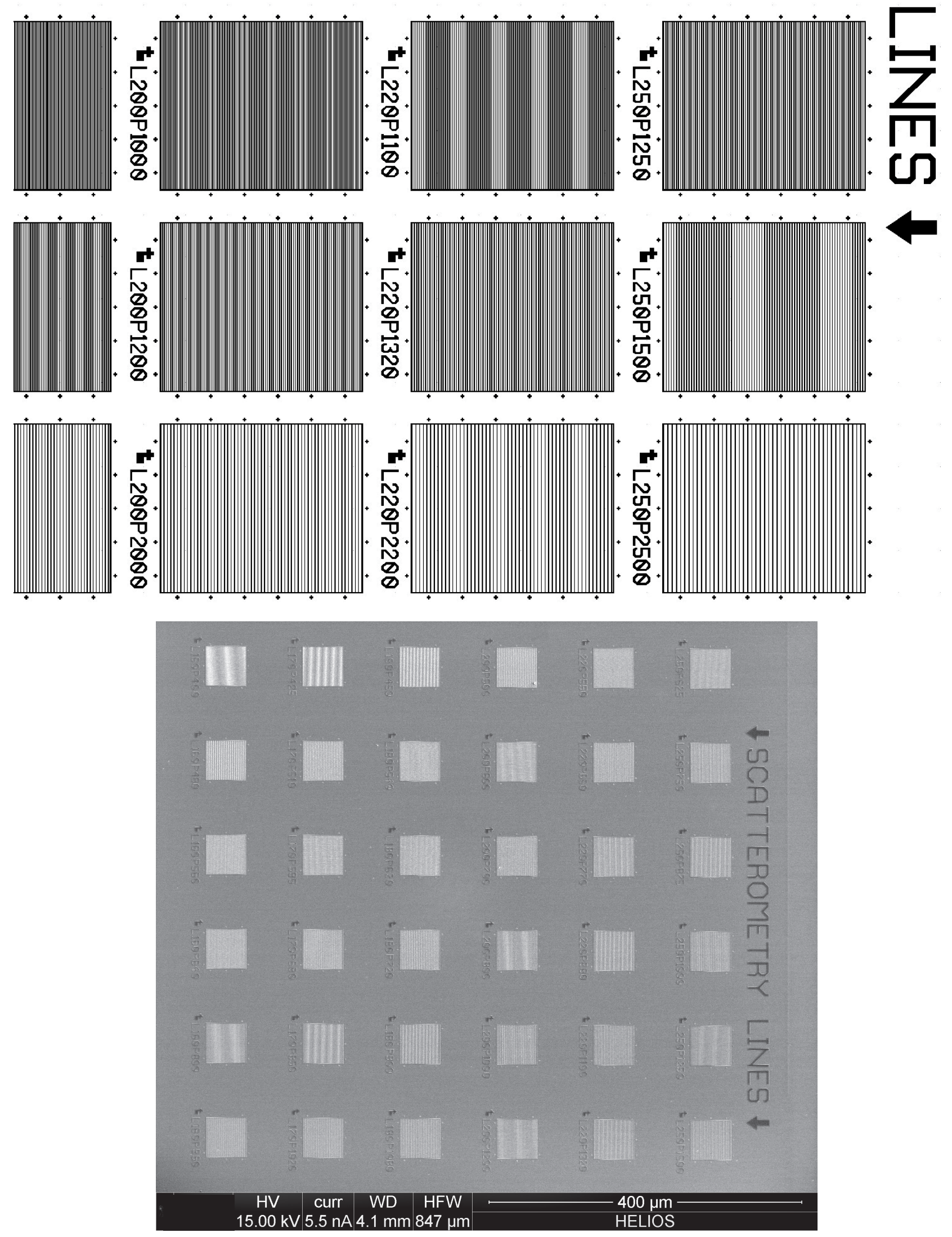

Plate 52 - Pattern 4-1 - Scatterometry Lines 
Plate 53 - Pattern 4-1 - Scatterometry Lines. (top) GDS II drawing of Pattern 4-1 at a smaller FOV showing some of the detail of the $250 \mathrm{~nm}$ lines and $750 \mathrm{~nm}$ pitch spacing pattern. (bottom) SEM micrograph of a similar area. 

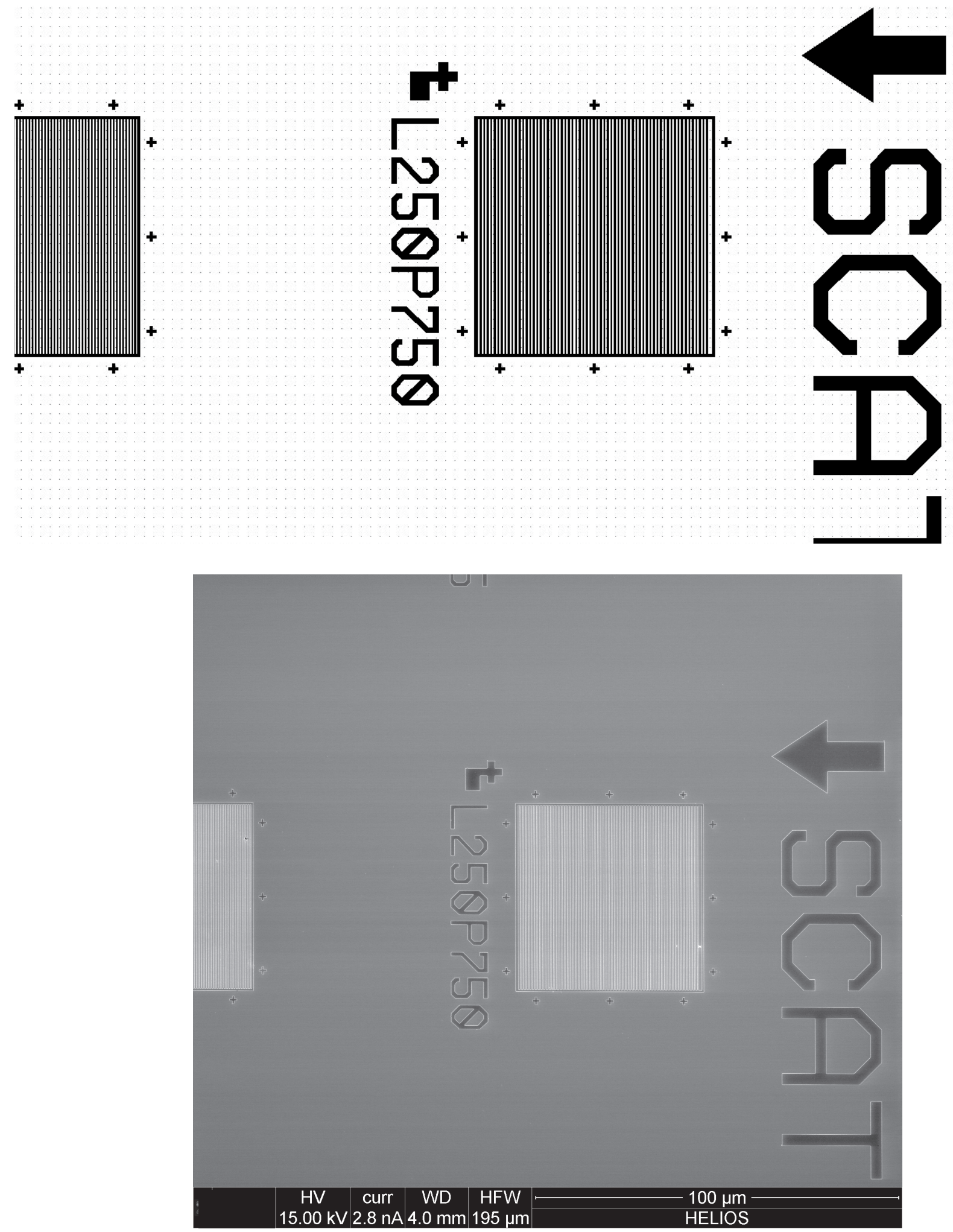

Plate 53 - Section 4; Pattern 4-1 
Plate 54 - Pattern 4-2 - Scatterometry 2 Series Cells. (top) GDS II drawing of the scatterometry lines Pattern 4-2 at wide FOV. (bottom) SEM micrograph of a similar area. 
SCATTEROMETRY2 - SERIES CELLS

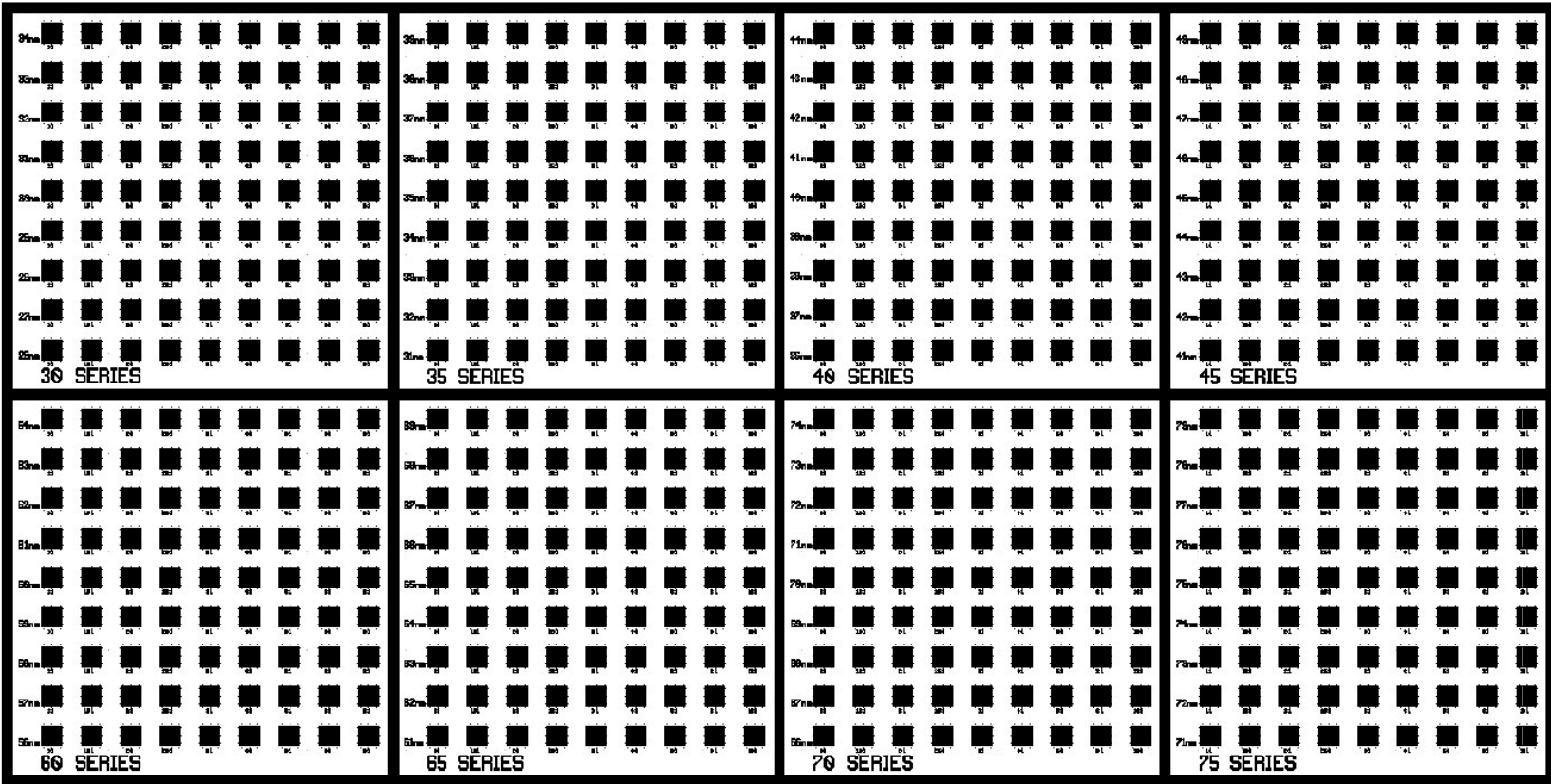

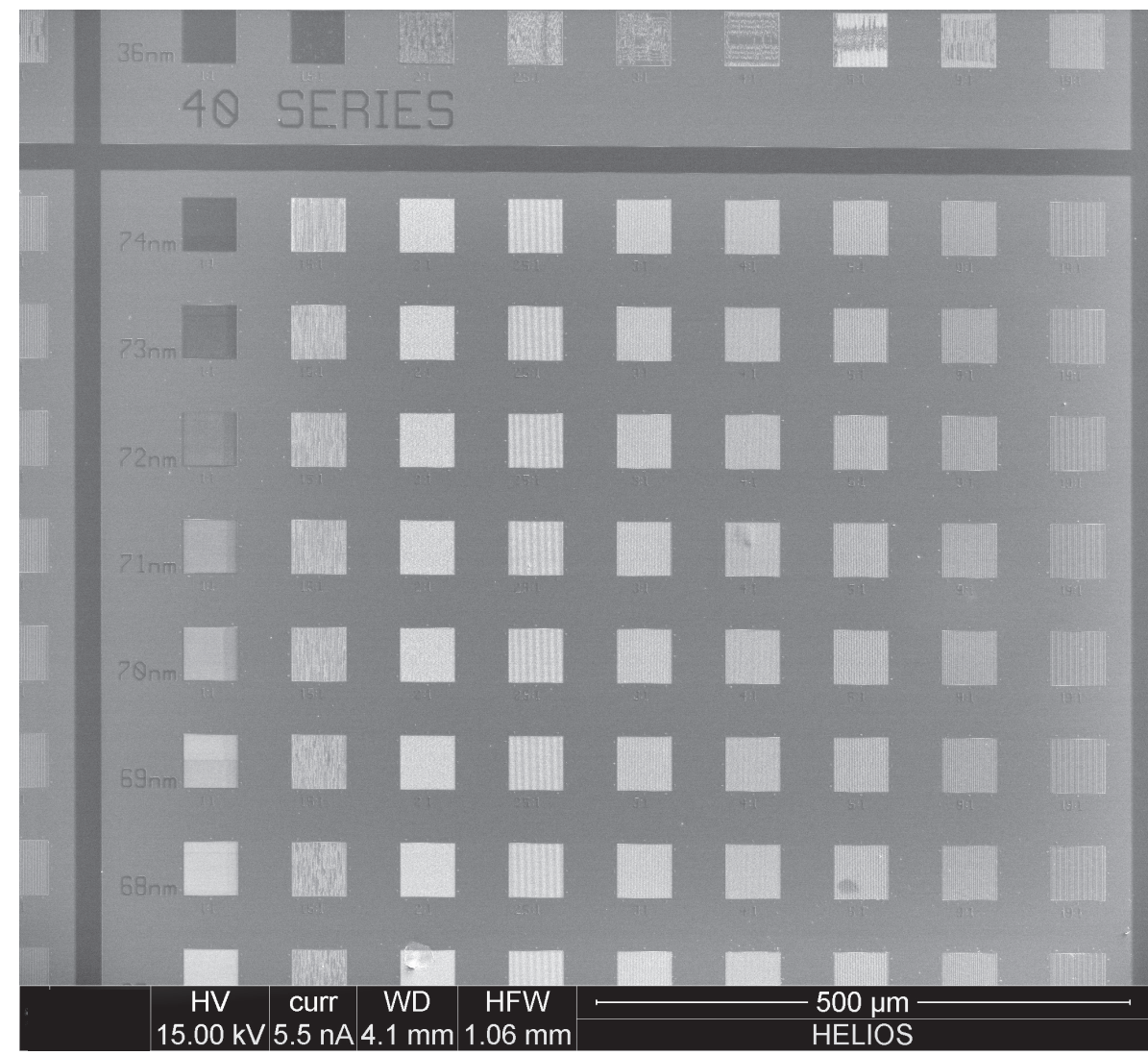

Plate 54 - Pattern 4-2 - Scatterometry 2 Series Cells. 
Plate 55 - Pattern 4-2 - Scatterometry 2 Series Cells. (top) GDS II drawing of Pattern 4-2 at a smaller FOV showing some of the detail of the fine patterns. (bottom) SEM micrograph of a similar area. 

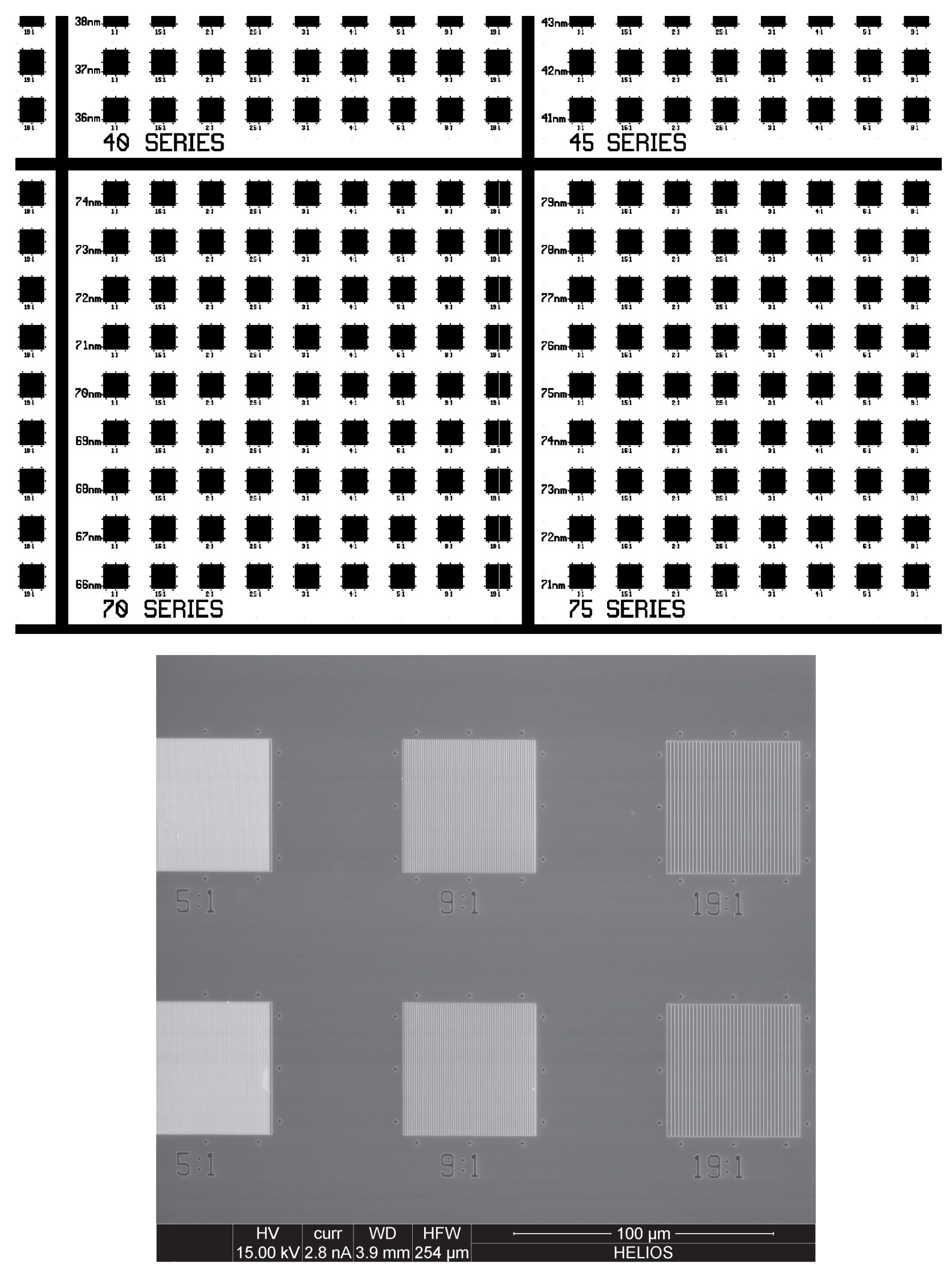

Plate 55 - Pattern 4-2 - Scatterometry 2 Series Cells. 
Plate 56 - Pattern 4-2 - Scatterometry 2 Series Cells. (top) GDS II drawing of the scatterometry lines Pattern 4-2 at smaller FOV showing the variety of line size and line pitches available. (bottom) SEM micrograph of a similar area. 


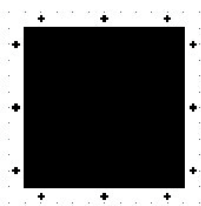

$3: 1$

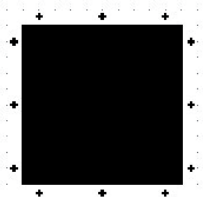

3:1

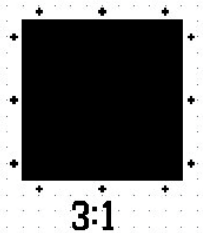

3:1

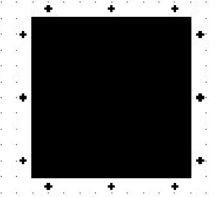

$4: 1$

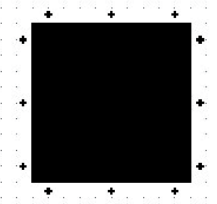

$4: 1$

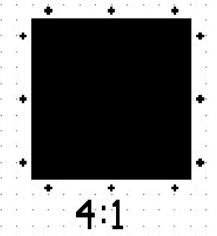

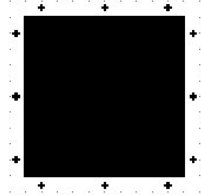

$5: 1$

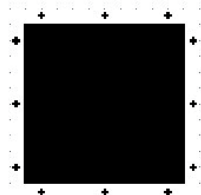

$5: 1$

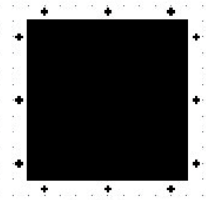

$5: 1$

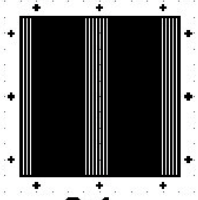

9:1

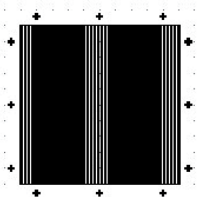

9:1

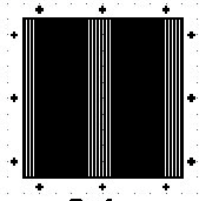

9:1

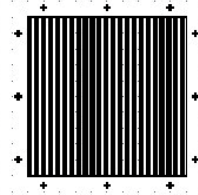

19:1

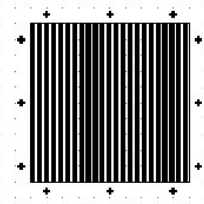

$19: 1$

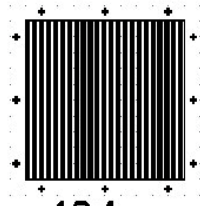

$19: 1$

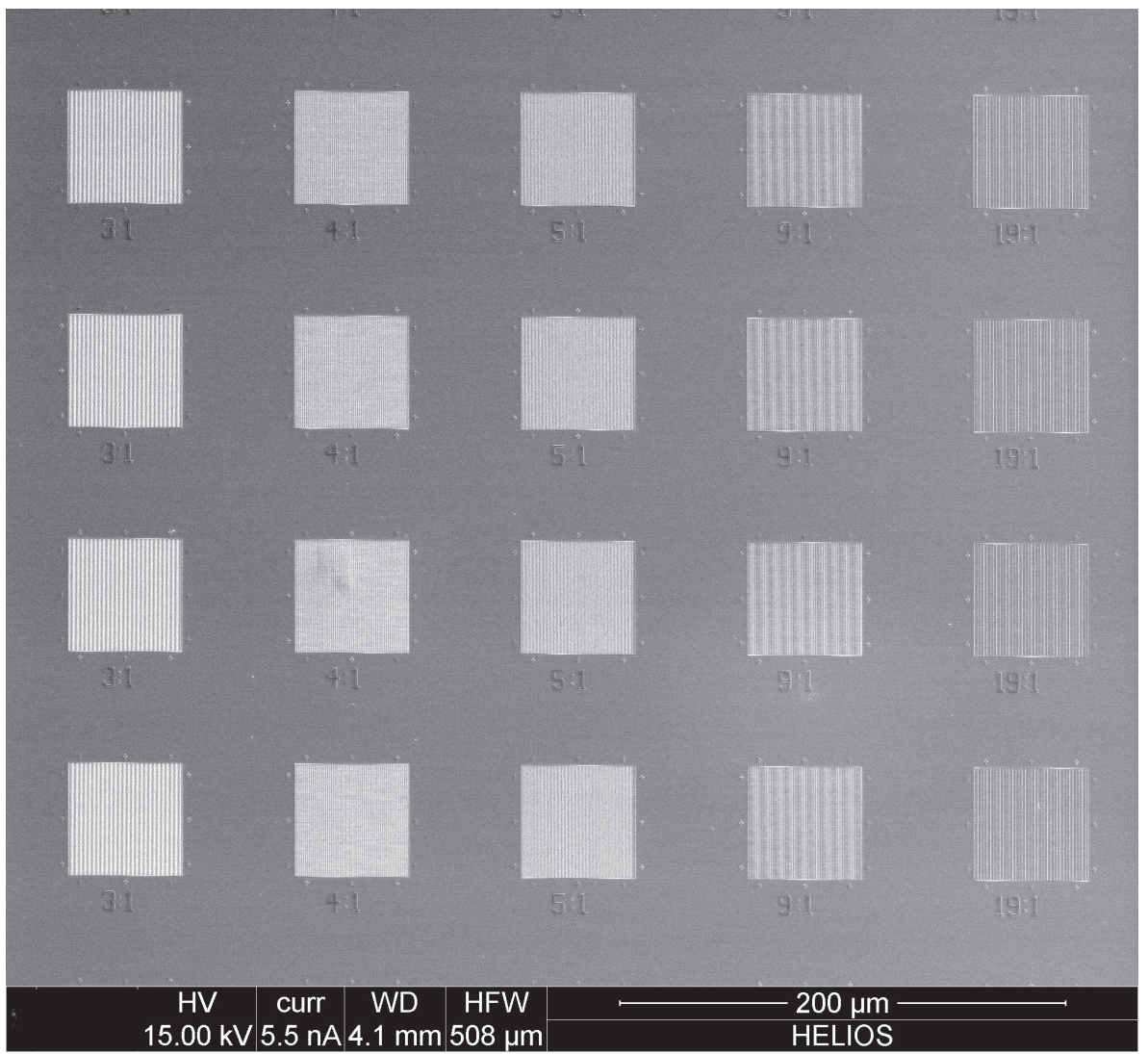

Plate 56 - Pattern 4-2- Scatterometry 2 Series Cells. 
Plate 57 - Pattern 4-2 - Scatterometry 2 Series Cells. (top) GDS II drawing of the scatterometry lines Pattern 4-2 at smaller FOV showing the variety of line size and line pitches available. (bottom) SEM micrograph of a similar area. 


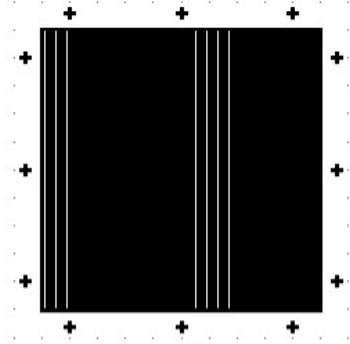

$5: 1$

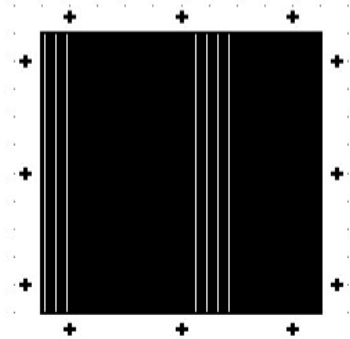

$5: 1$

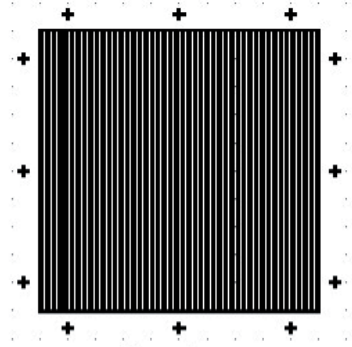

9:1

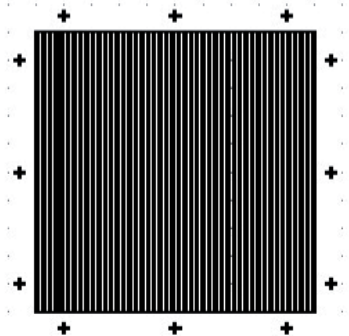

$9: 1$
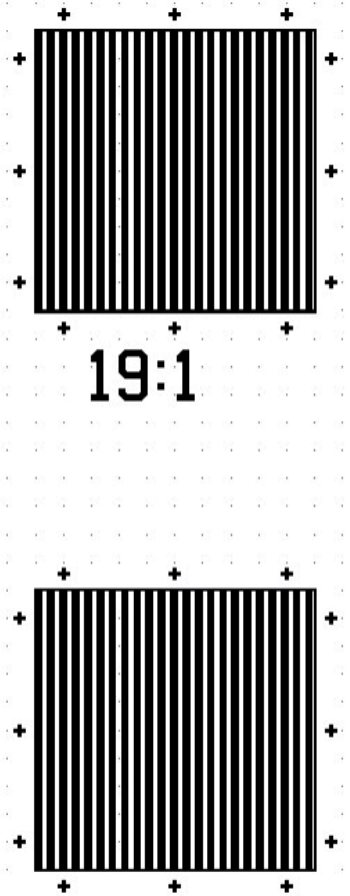

19:1

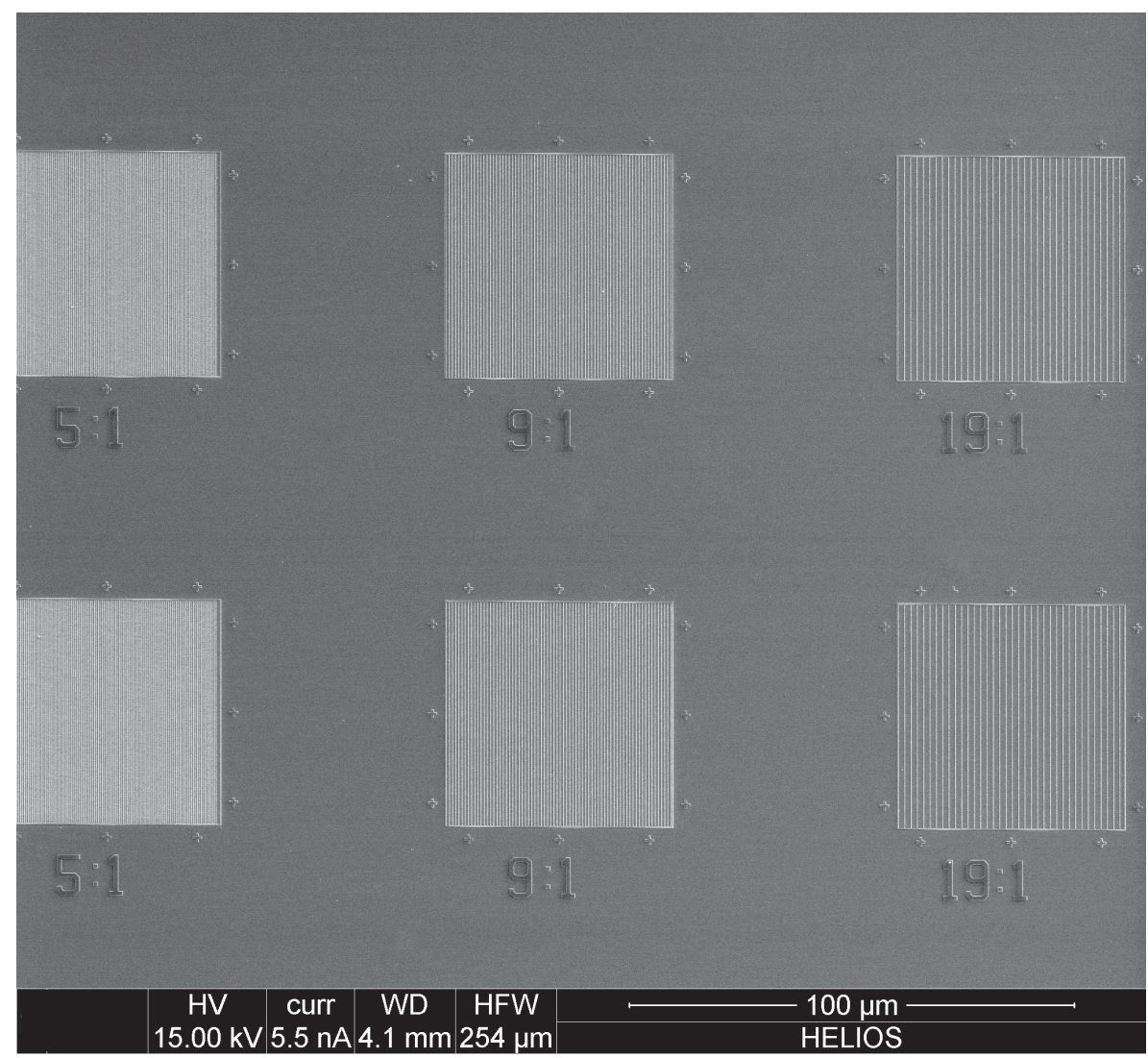

Plate 57 - Pattern 4-2 - Scatterometry 2 Series Cells. 
Plate 58 - Pattern 4-3 and 4-4 - Scatterometry Lines and Trenches. (top) GDS II drawing of the scatterometry lines and trenches of Patterns 4-3 and 4-4 at wide FOV. (bottom) SEM micrograph of a similar area. 

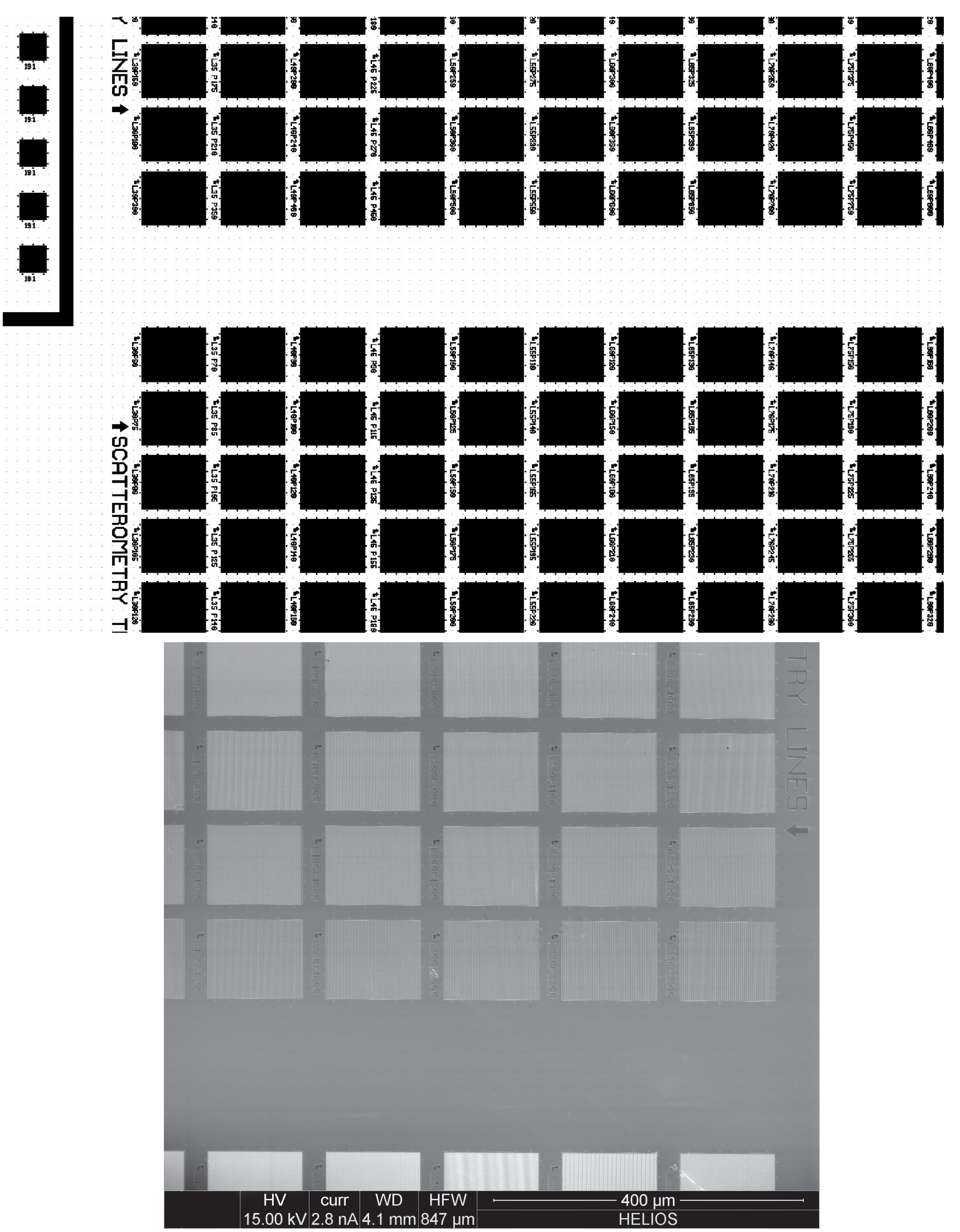

Plate 58 - Pattern 4-3 and 4-4 - Scatterometry Lines and Trenches. 
Plate 59 - Pattern 4-3 - Scatterometry Lines. (top) GDS II drawing of Pattern 4-3 at a smaller FOV showing some of the detail of the fine patterns. (bottom) SEM micrograph of several of the test patterns. 

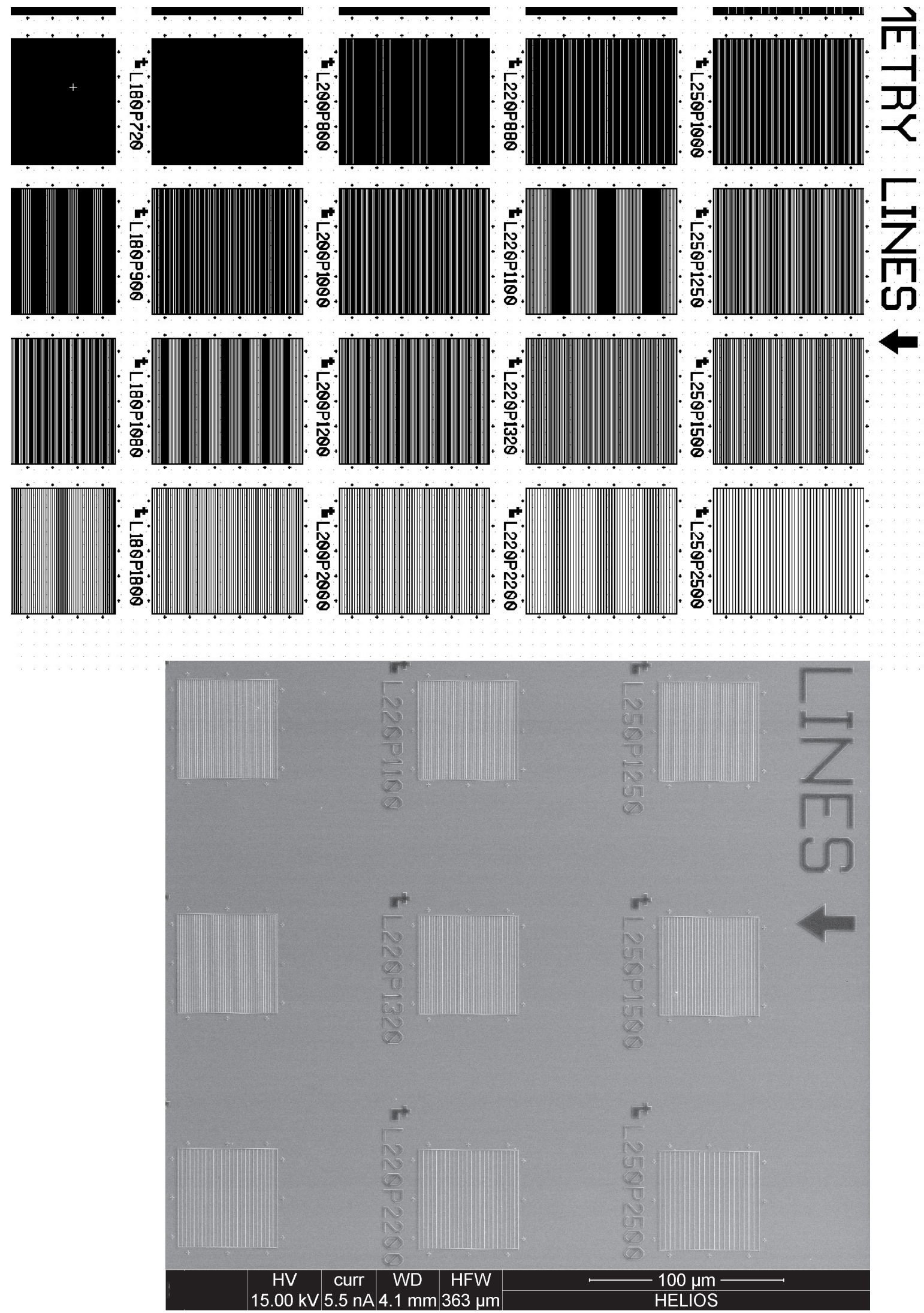

Plate 59 - Pattern 4-3 Scatterometry Lines. 
Plate 60 - Pattern 4-3 - Scatterometry Lines. (top) GDS II drawing of Pattern 4-3 at a smaller FOV showing some of the detail of the fine patterns. (bottom) SEM micrograph of the L250; P1500 pattern. 

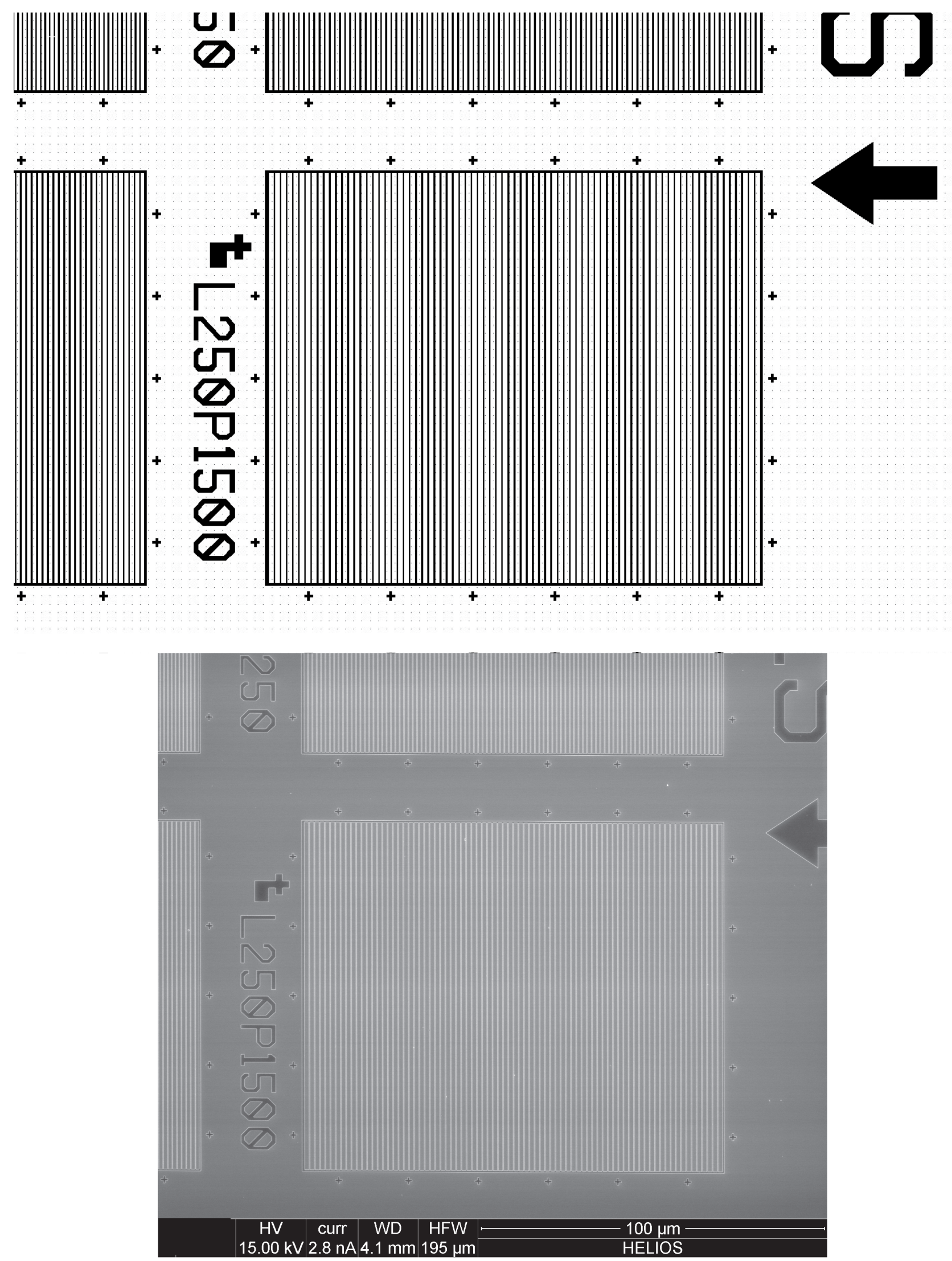

Plate 60 - Pattern 4-3 Scatterometry Lines. 
Plate 61 - Pattern 4-4 - Scatterometry Trenches. (top) GDS II drawing of Pattern 4-4 showing some of the detail of the fine patterns. (bottom) SEM micrograph of a similar area of the pattern. 

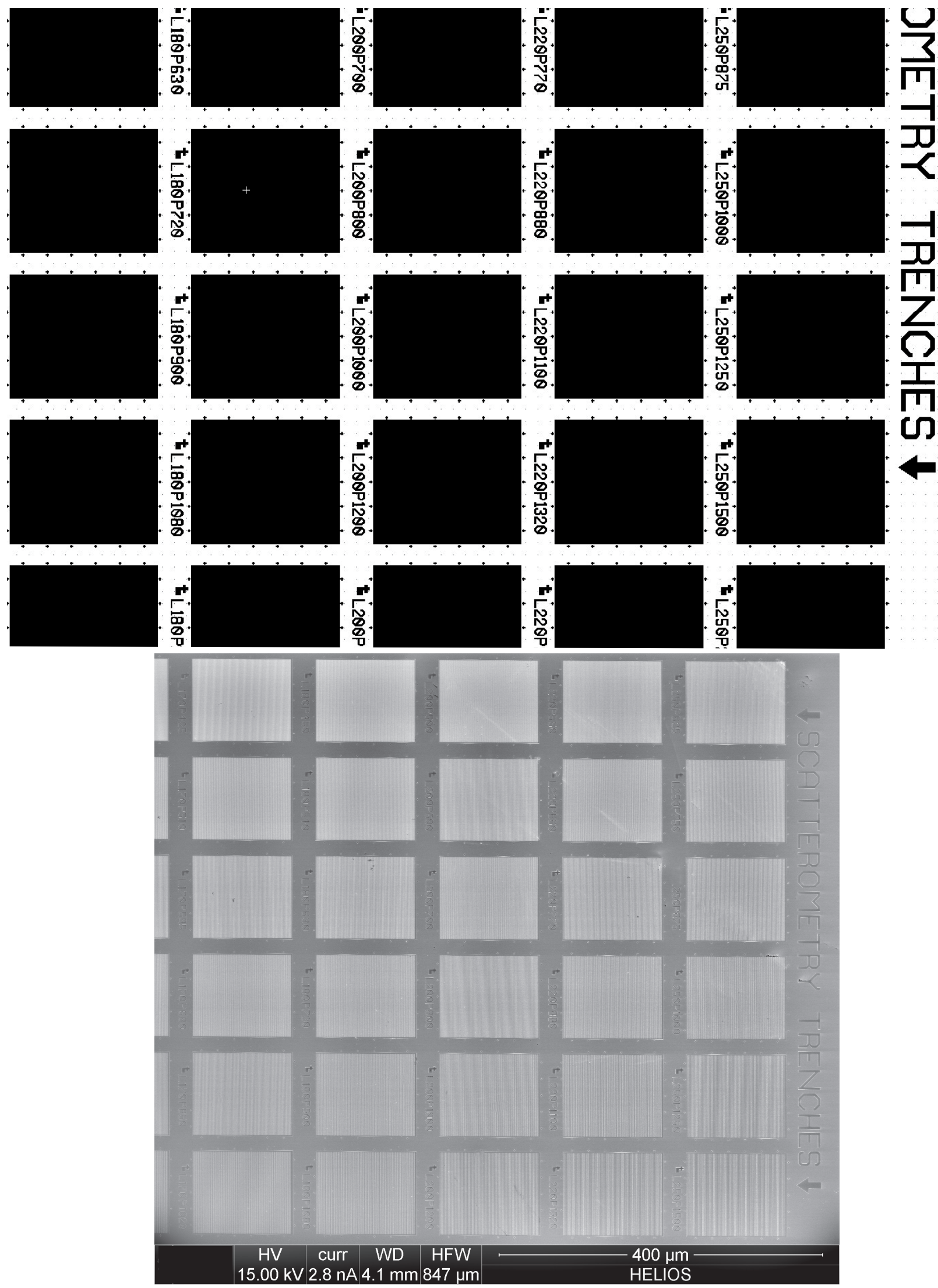

Plate 61 - Pattern 4-4 Scatterometry Trenches 
Plate 62 - Pattern 4-4 - Scatterometry Trenches. (top) GDS II drawing of Pattern 4-4 at a smaller FOV showing some of the detail of the fine patterns. (bottom) SEM micrograph of the L250; P 750 pattern. 

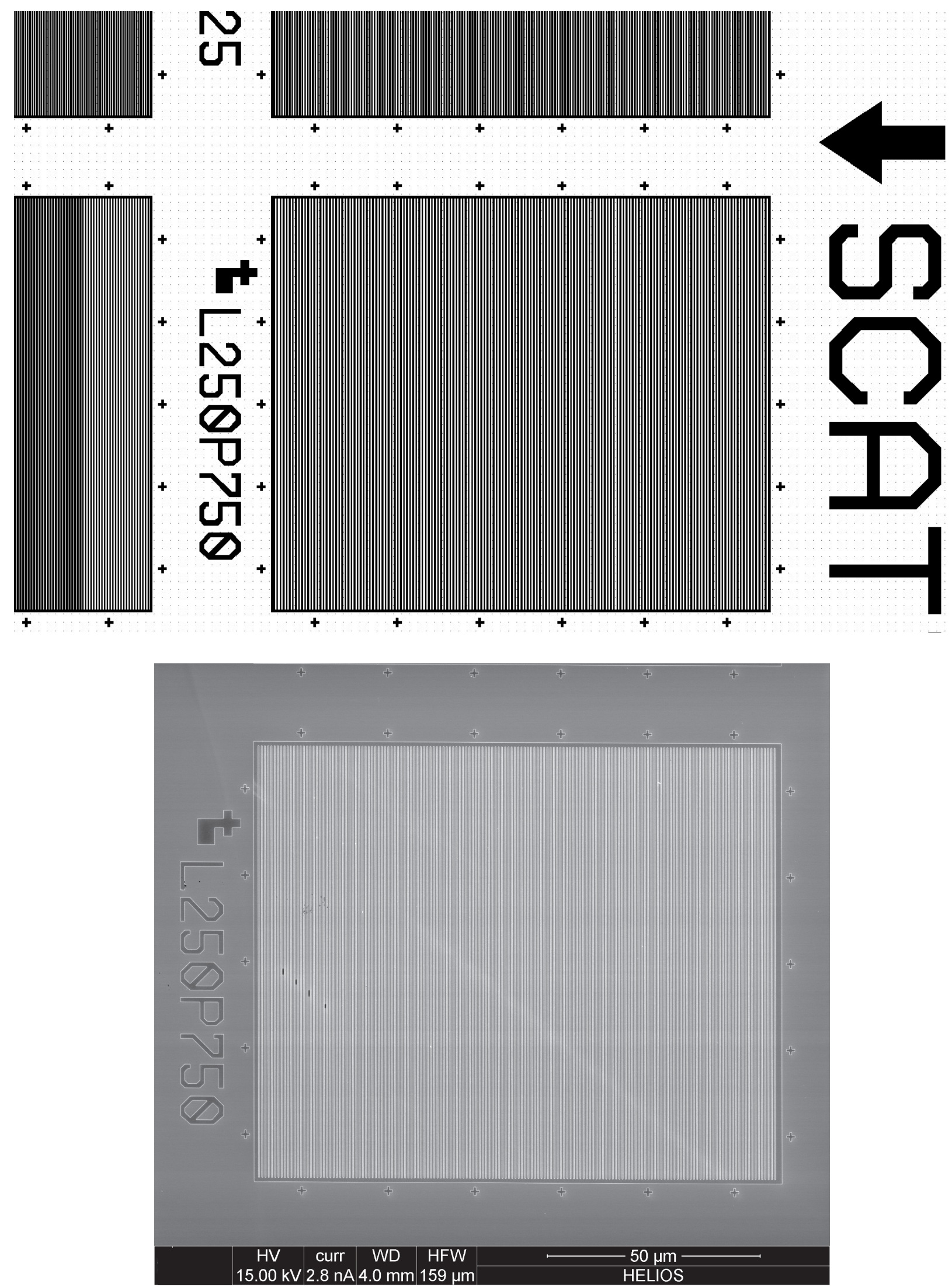

Plate 62 - Pattern 4-4 - Scatterometry Trenches. 
Page intentionally left blank 
SECTION 5 
Plate 63. Section 5-0 - Overall. (top) GDS II line drawing of the entire RM 8820 pattern highlighting Section 5 with the 10 additional major sub-patterns numerically identified. (bottom) GDS II detail drawing of Section 5 showing the 10 additional major sub-patterns included in this section. 

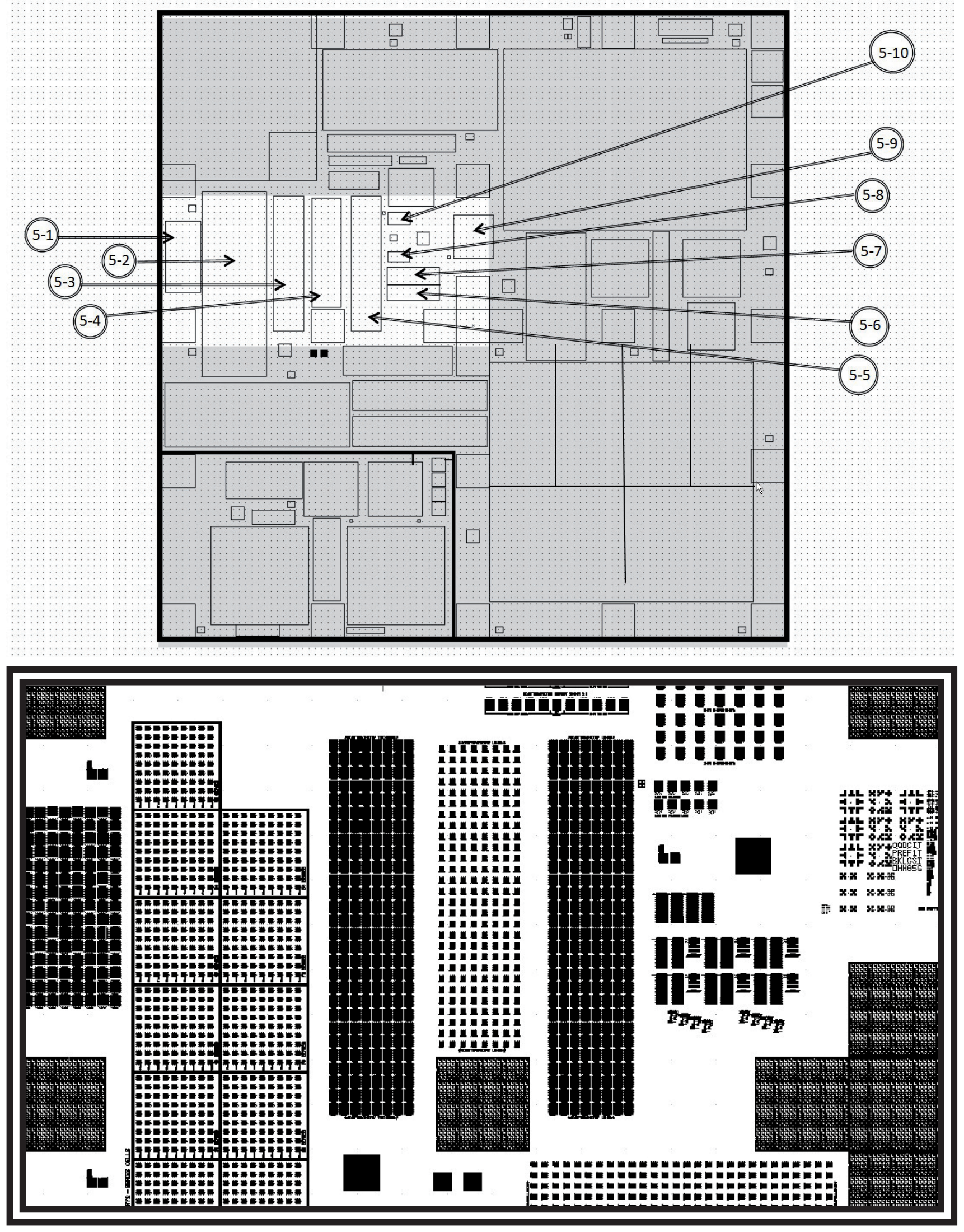

Plate 63. Section 5-0 - Overall 
Plate 64 - Pattern 5-1 - Contact Holes. (top) GDS II drawing of the contact holes Pattern 5-1 at wide FOV. (bottom) SEM micrograph of a similar area. 

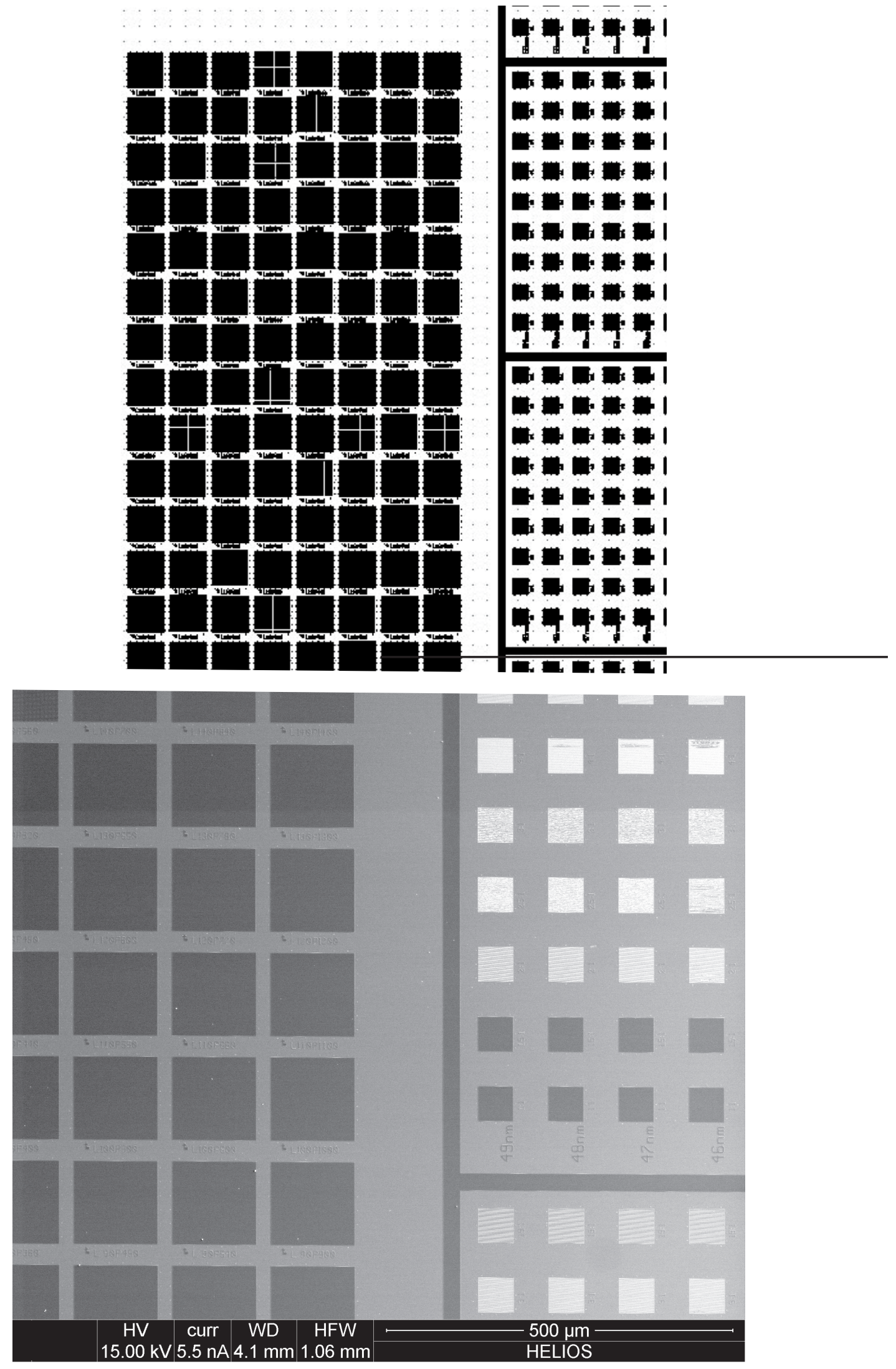

Plate 64 - Pattern 5-1 - Contact Holes 
Plate 65 - Pattern 5-1 - Contact Holes. (top) GDS II drawing of the contact holes Pattern 5-1 a smaller FOV showing some of the detail of the fine patterns. (bottom) SEM micrograph of a similar area. 

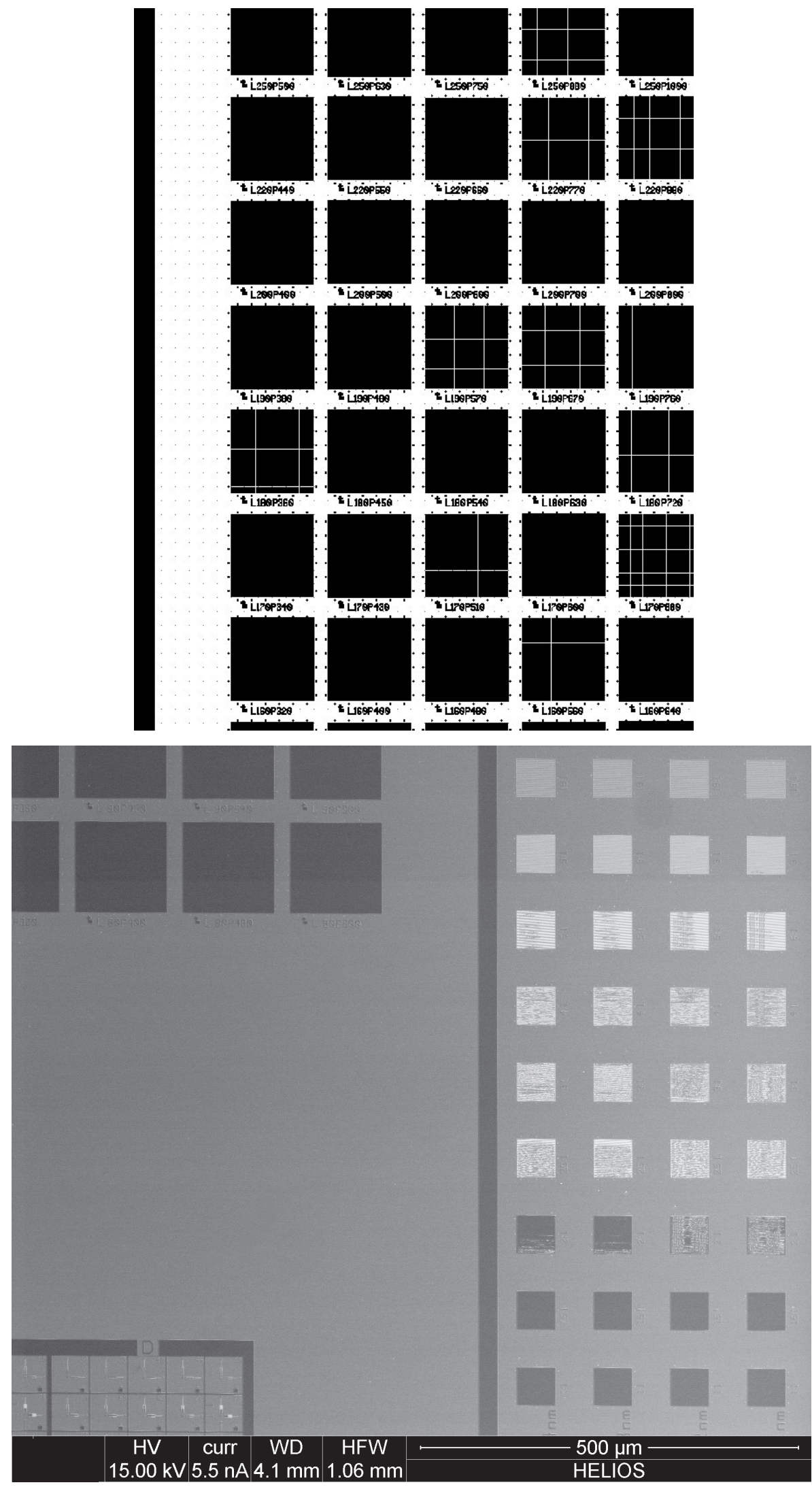

Plate 65 - Pattern 5-1 - Contact Holes 
Plate 66 - Pattern 5-1 - Contact Holes. (top) GDS II drawing of Pattern 5-1 at a reduced FOV showing some of the detail of the fine pattern with a nominal $250 \mathrm{~nm}$ contact holes and $880 \mathrm{~nm}$ pitch. (bottom) SEM micrograph of a similar area. 

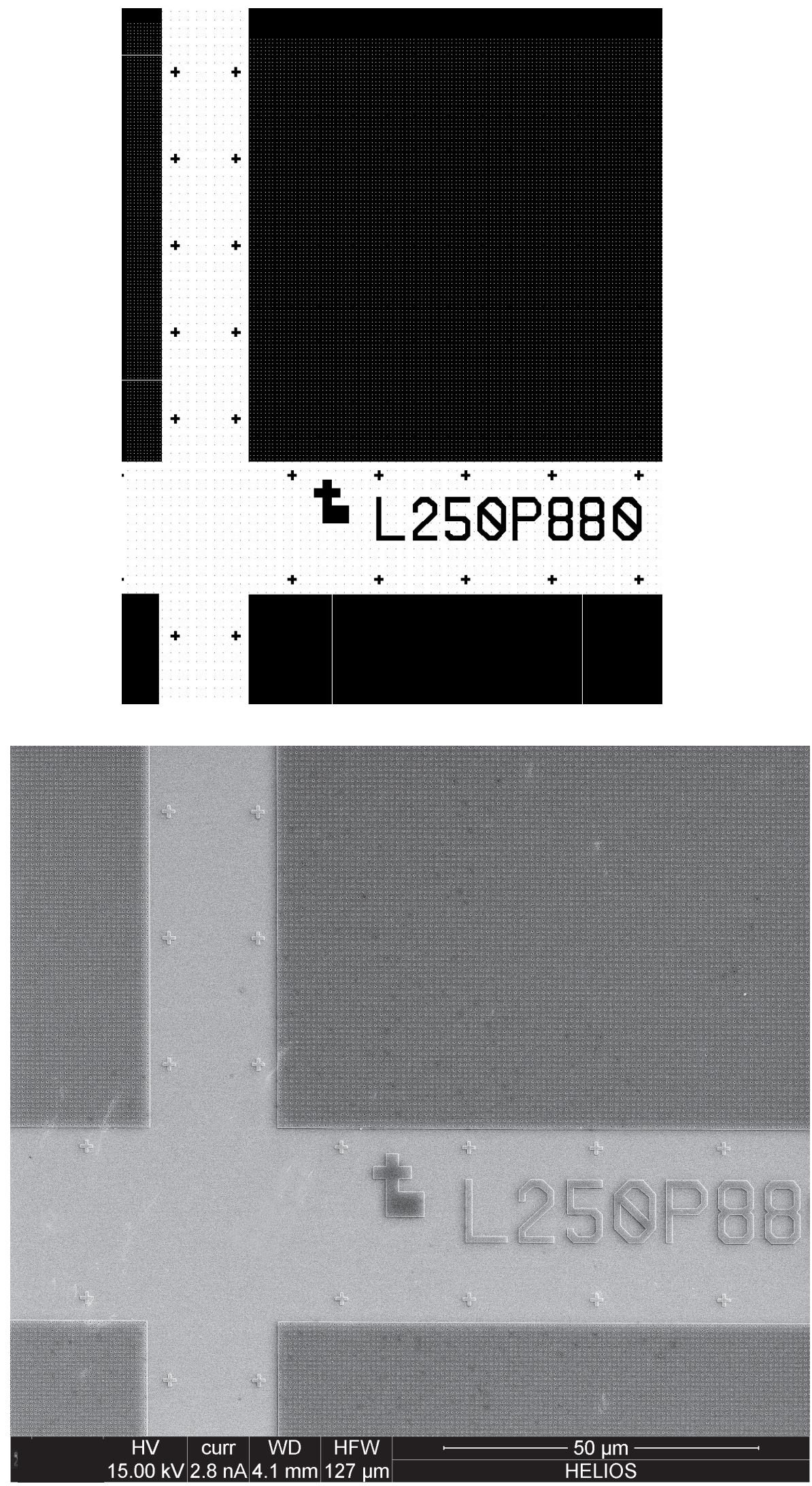

Plate 66 - Pattern 5-1 - Contact Holes 
Plate 67 - Pattern 5-1 - Contact holes. (top) SEM micrograph of contact hole pattern of the contact holes Pattern 5-1 at wide FOV. (bottom) SEM micrograph of a smaller FOV showing some of the detail of the fine details of Pattern 5-1. 

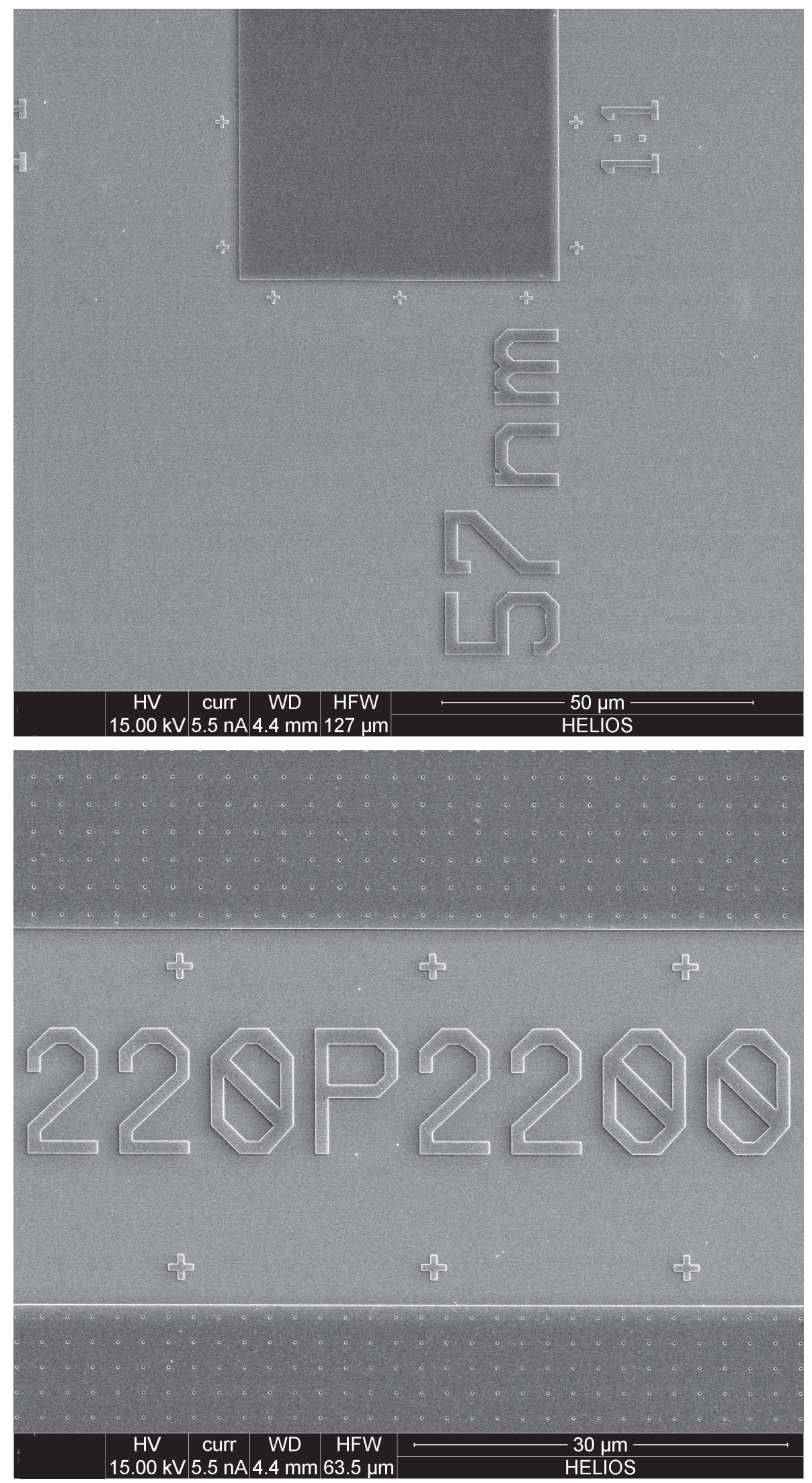

Plate 67 - Pattern 5-1 - Contact holes 
Plate 68 - Pattern 5-2 - Scatterometry 2 Sseries Cells. GDS II drawing of the scatterometry lines Pattern 5-2 at wide FOV. 


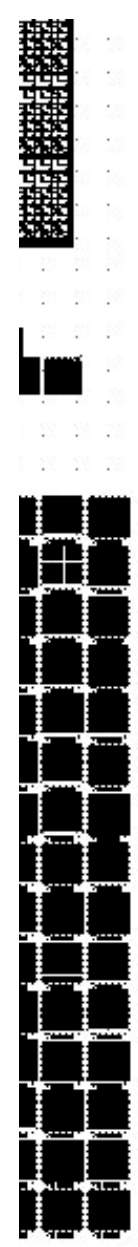

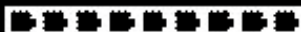
E

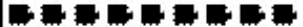

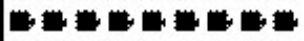

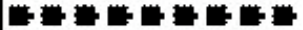

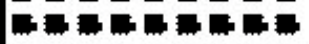

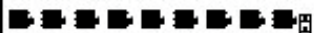

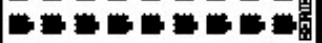

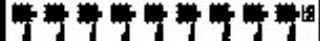

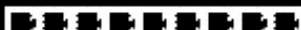

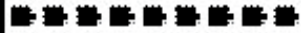
E

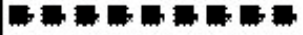

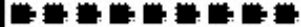

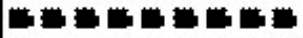
둔

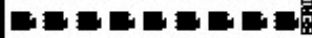

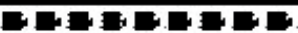

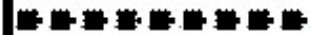
E

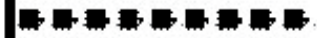

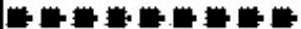

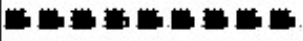
E

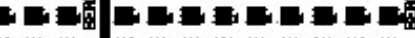

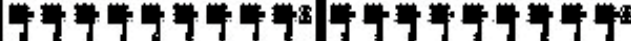

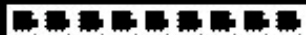

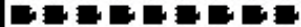

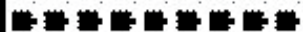

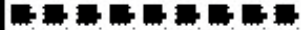

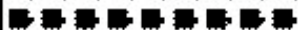

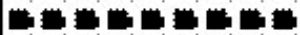

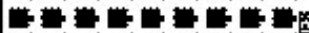

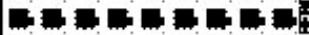

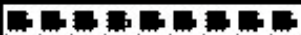

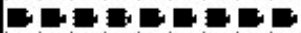

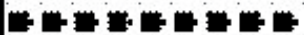
En

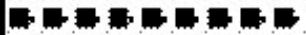

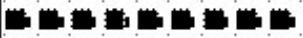

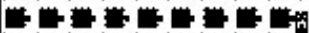

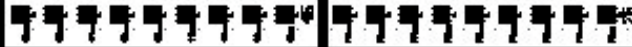

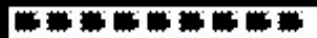

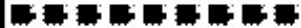

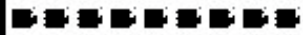

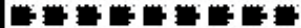
E

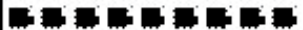

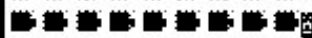

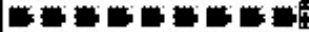

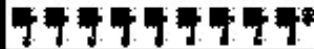

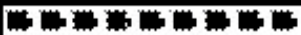
E E

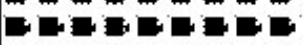

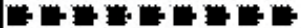

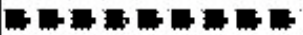

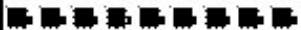

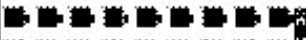

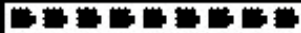

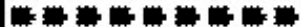

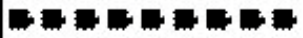

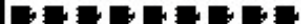

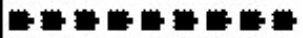

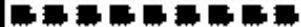

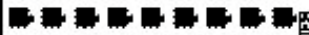

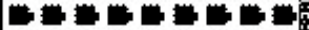

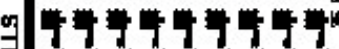

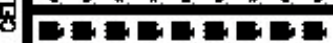

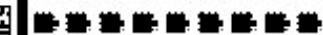

f

I

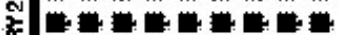

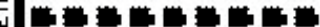

进

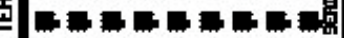

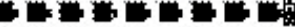

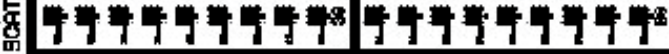

ES CELLS 
Plate 69 - Pattern 5-2 - Scatterometry 2 Series Cells. (top) GDS II drawing of the scatterometry lines Pattern 5-2 at wide FOV. (bottom) GDS II drawing of Pattern 5-2 at a reduced FOV showing some of the fine detail of a pattern with 19:1 ratio. 


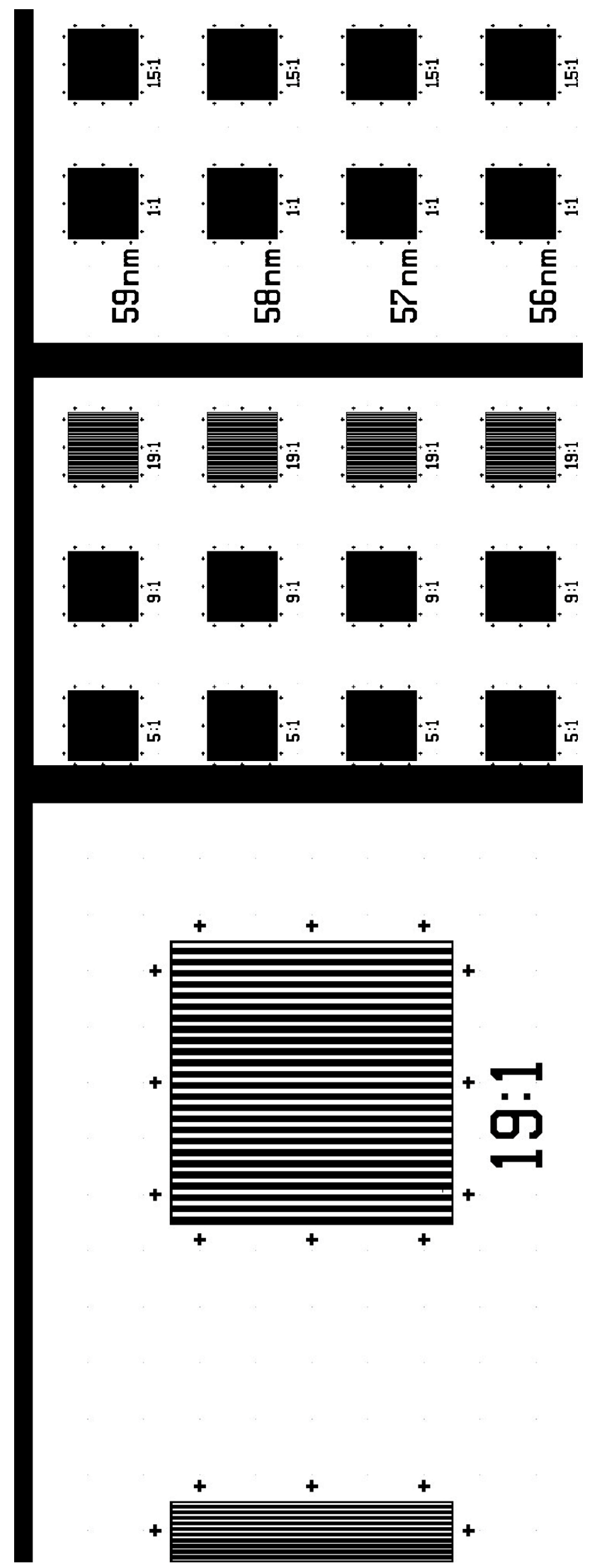

Plate 69 - Pattern 5-2 - Scatterometry 2 Series Cells 
Plate 70 - Pattern 5-2 - Scatterometry 2 Series Cells. (top) SEM micrograph of Pattern 5-2 at wide HFW. (bottom) SEM micrograph of Pattern 5-2 at a reduced HFW showing some of the fine detail of a pattern with 19:1 ratio. 

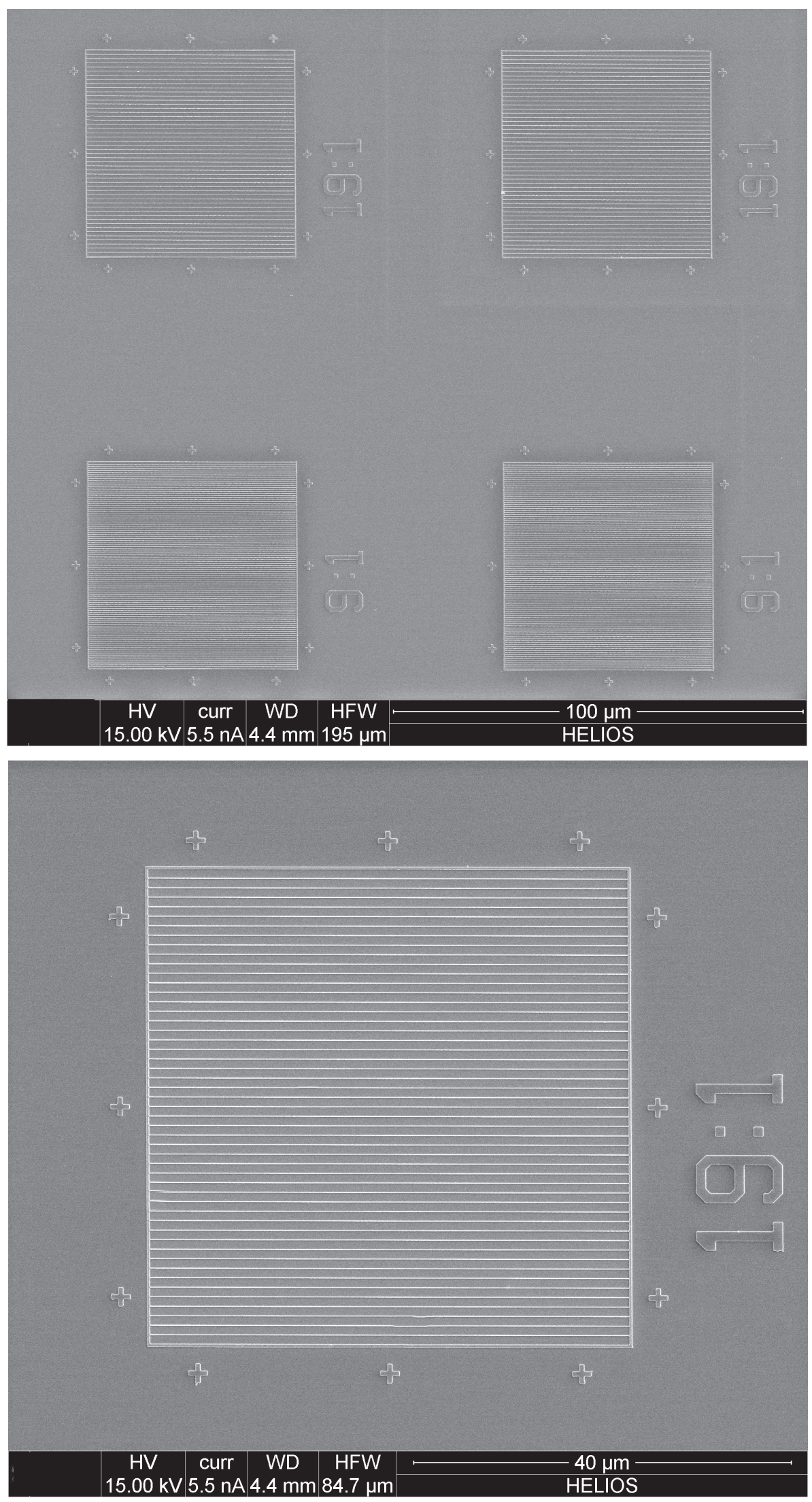

Plate 70 - Pattern 5-2 - Scatterometry 2 series cells 
Plate 71 - Patterns 5-3, 5-4, and 5-5 - Scatterometry. GDS II drawing of the scatterometry lines Pattern 5-3, 5-4, 5-5 and 5-6 at wide FOV. 


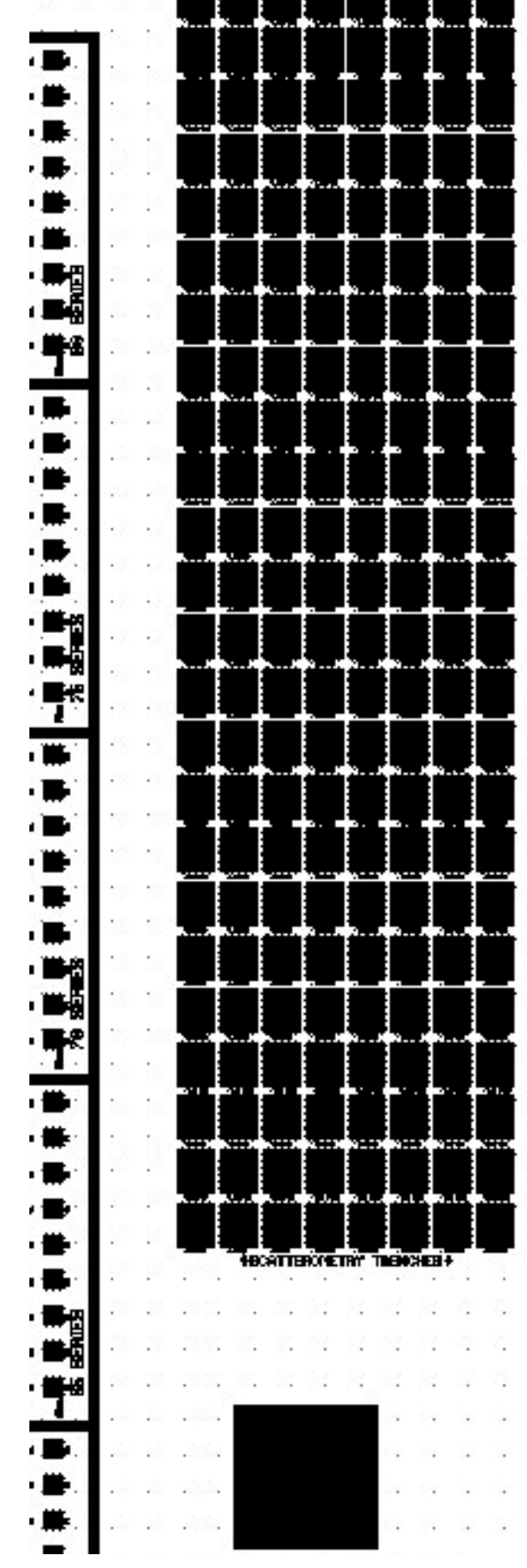

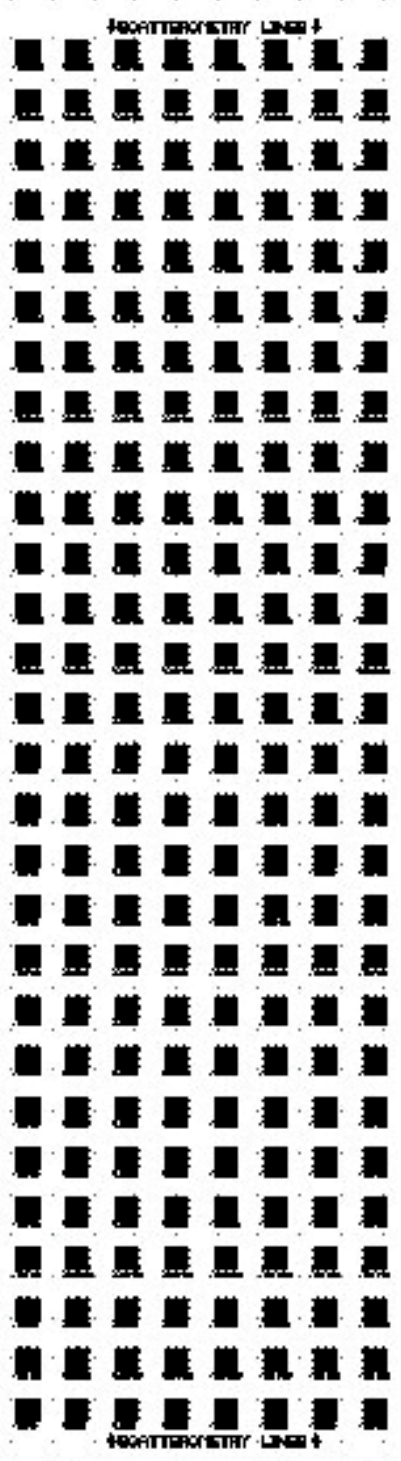
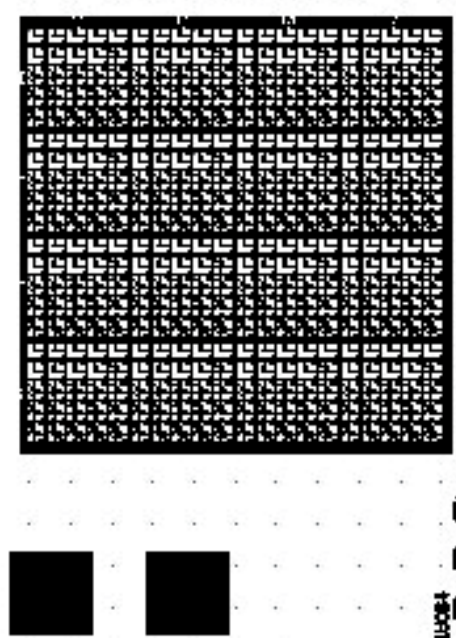

ש
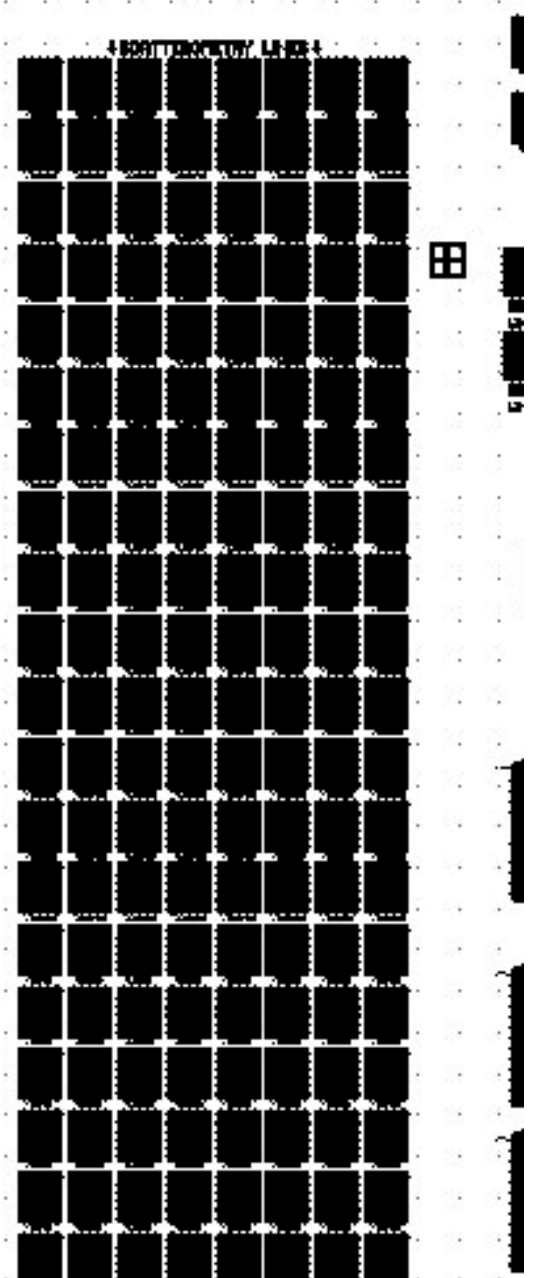

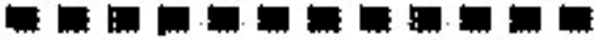

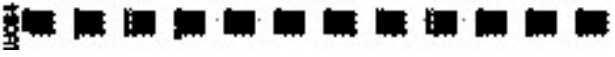

Plate 71 - Patterns 5-3, 5-4, and 5-5 - Scatterometry 
Plate 72 - Pattern 5-3 - Scatterometry trenches. GDS II drawing of the scatterometry trenches Pattern 5-3 at wide FOV. 

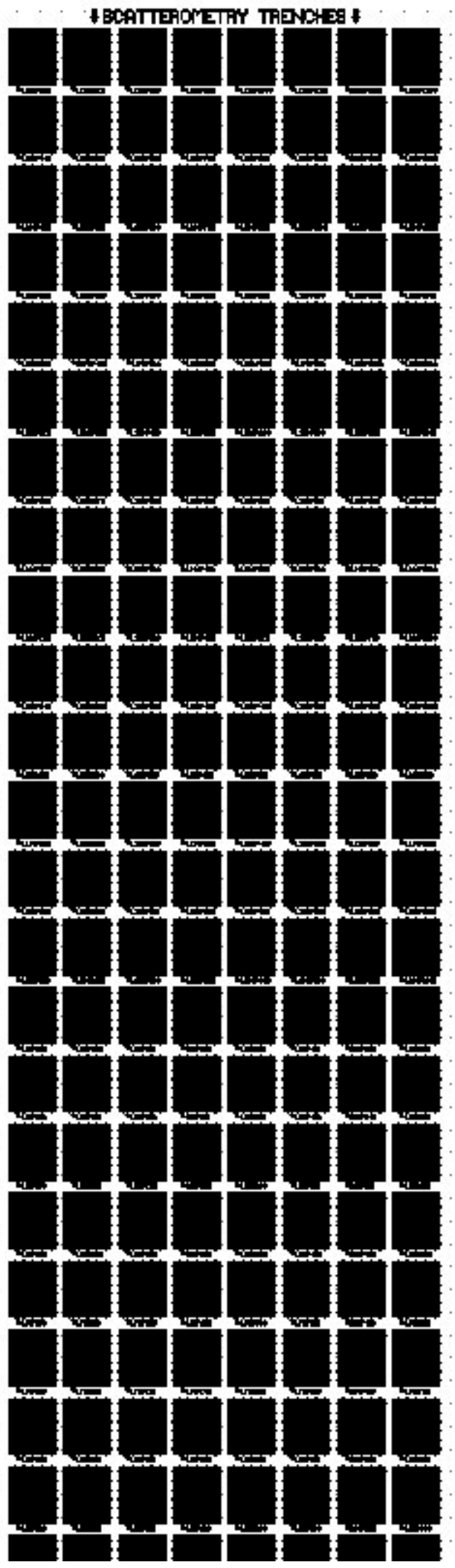

Plate 72 - Pattern 5-3 - Scatterometry trenches 
Plate 73 - Pattern 5-3 - Scatterometry trenches. (top) SEM micrograph of Pattern 5-3 at wide FOV (bottom) SEM micrograph of Pattern 5-3 at a smaller FOV showing some of the detail of the fine patterns. 

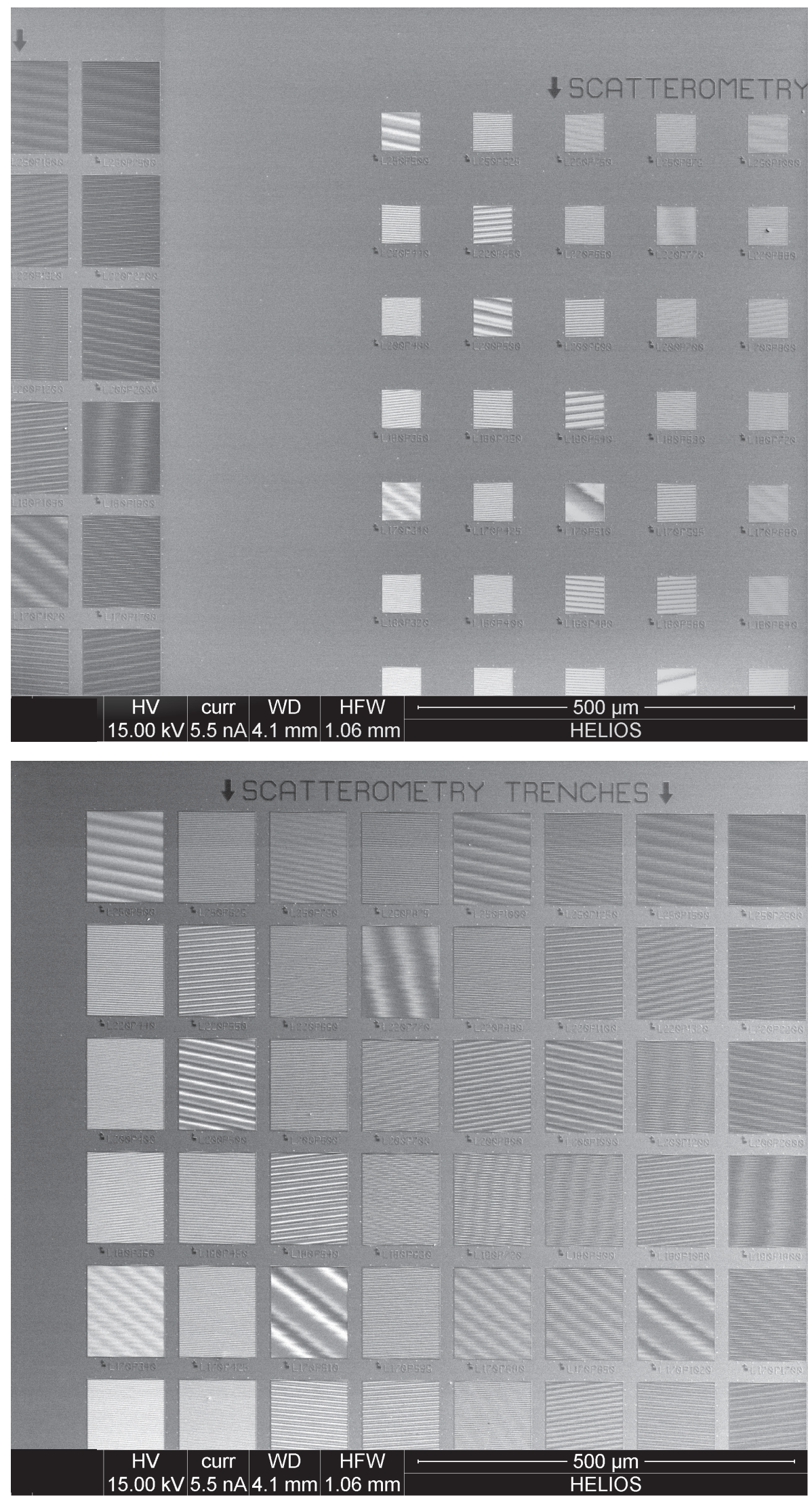

Plate 73 - Pattern 5-3 - Scatterometry trenches 
Plate 74 - Pattern 5-3 - Scatterometry trenches. (top) SEM micrograph of Pattern 5-3 at wide FOV (bottom) SEM micrograph of Pattern 5-3 at a smaller FOV showing some of the detail of the fine patterns. 

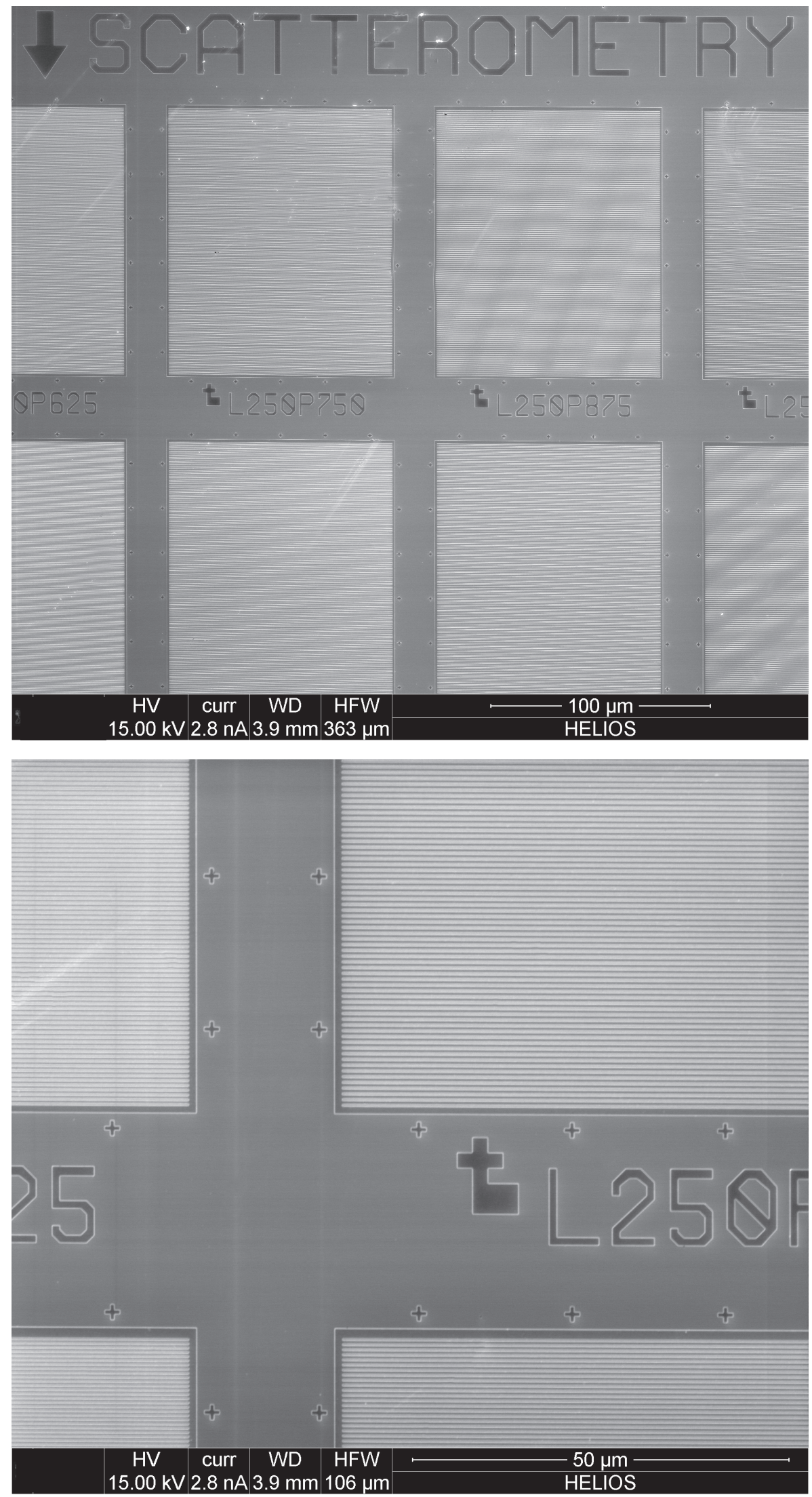

Plate 74 - Pattern 5-3 - Scatterometry trenches. 
Plate 75 - Pattern 5-4 - Scatterometry lines. GDS II drawing of the scatterometry lines Pattern 5-3 at wide FOV. 


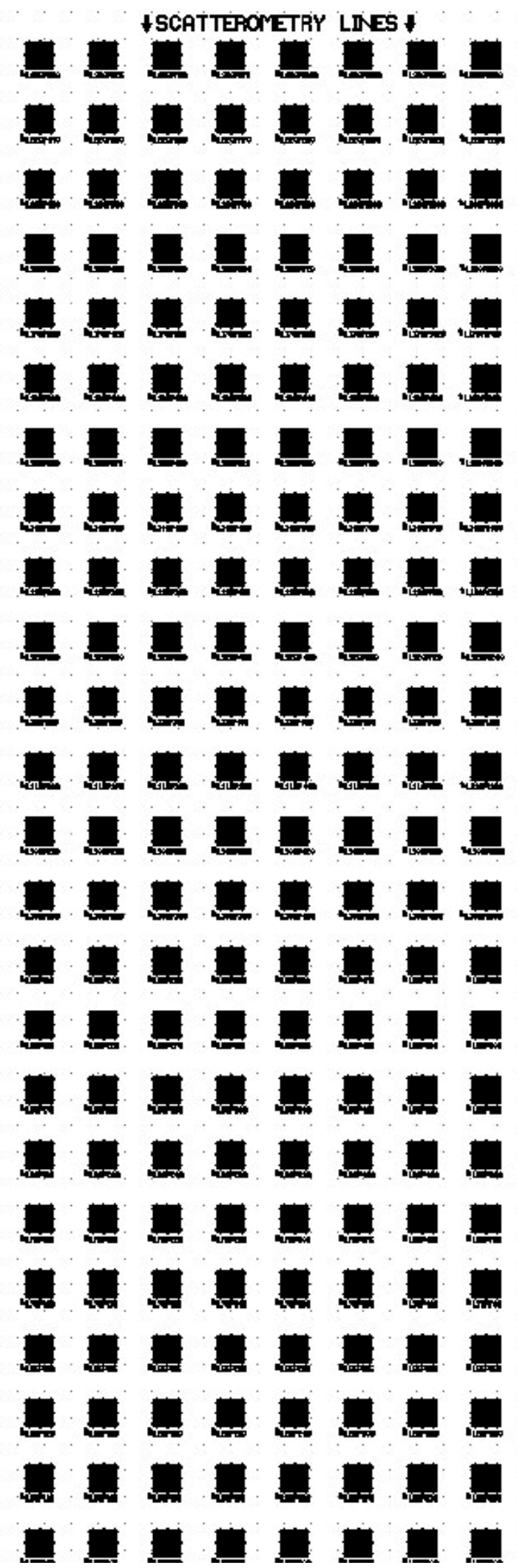

Plate 75 - Pattern 5-4 - Scatterometry lines 
Plate 76 - Pattern 5-4 - Scatterometry lines (top) GDS II drawing of the scatterometry lines Pattern 5-4 at wide FOV. (bottom) SEM micrograph of a similar area. 


\section{$\downarrow$ SCATTEROMETRY}
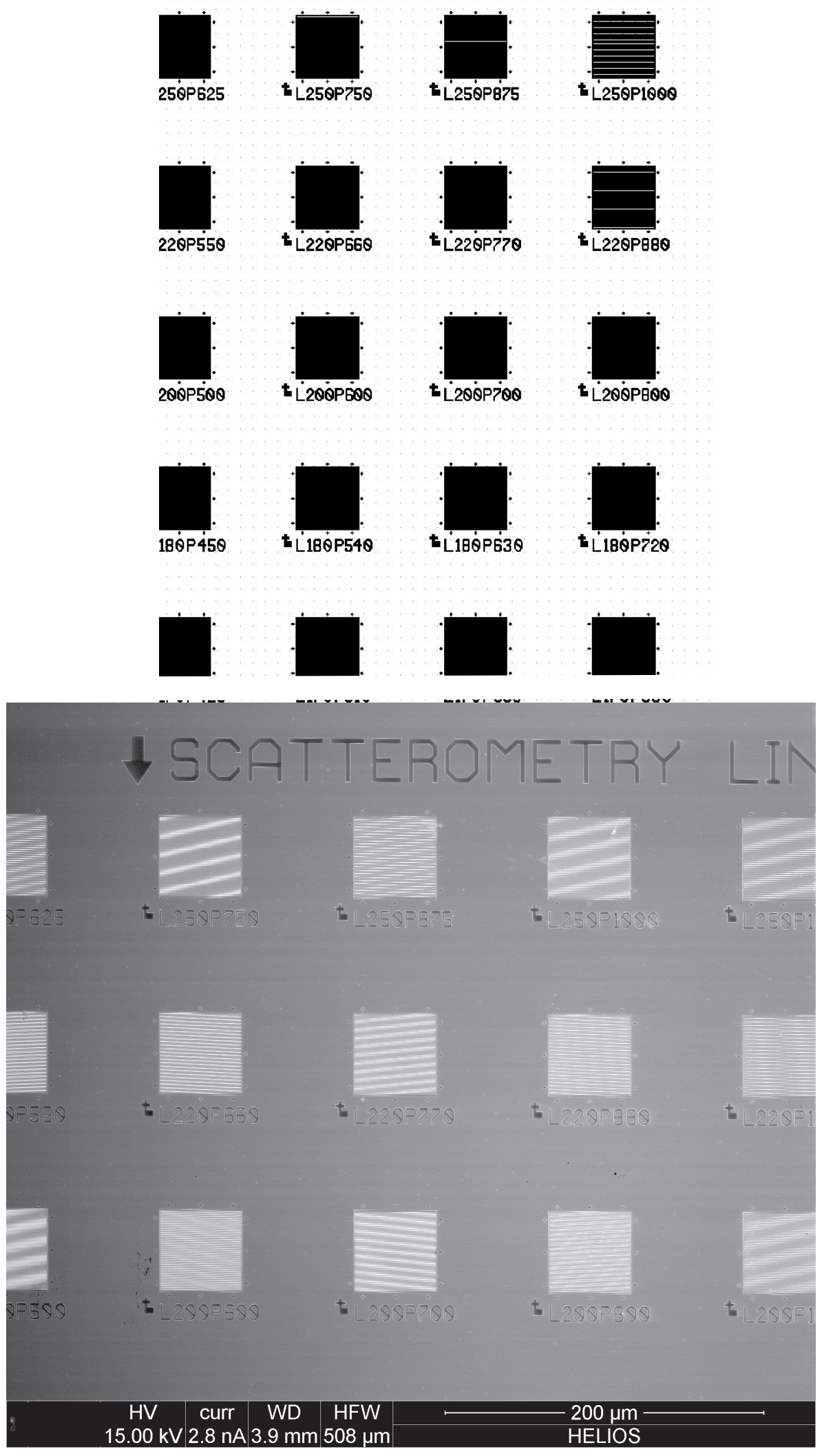

Plate 76 - Pattern 5-4 - Scatterometry lines 
Plate 77 - Pattern 5-4 - Scatterometry lines (top) GDS II drawing of the scatterometry lines Pattern 5-4 at wide FOV. (bottom) SEM micrograph of a similar area. 

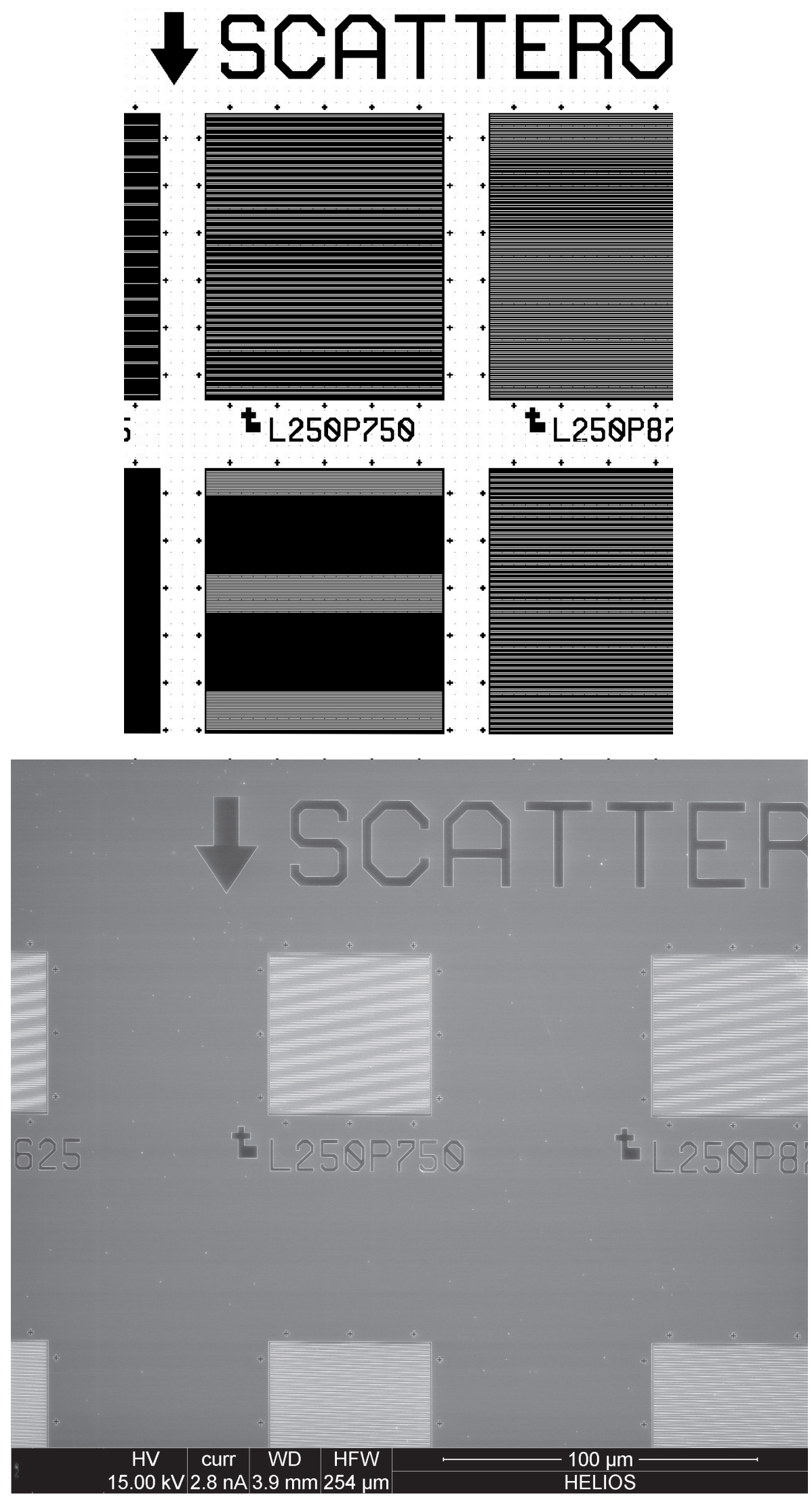

Plate 77 - Pattern 5-4 - Scatterometry lines 
Plate 78 - Pattern 5-5 - Scatterometry lines. GDS II drawing of the scatterometry lines Pattern 5-3 at wide FOV. 


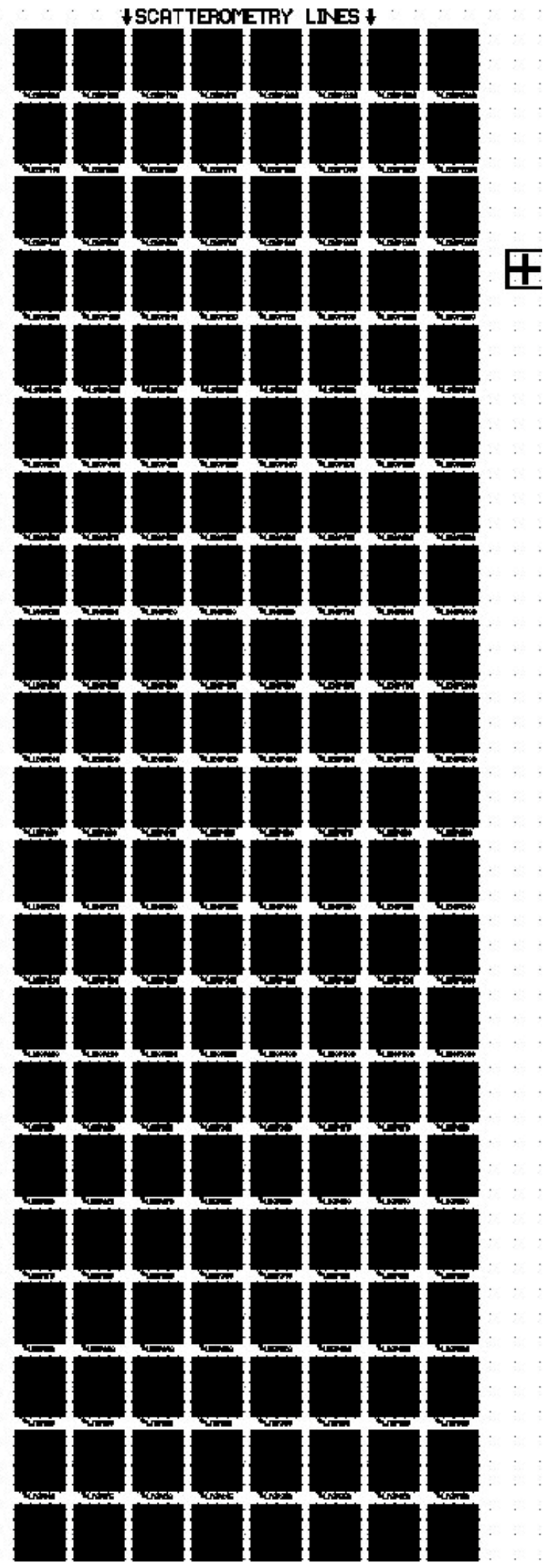

Plate 78 - Pattern 5-5 - Scatterometry lines 
Plate 79 - Pattern 5-5 - Scatterometry lines (top) GDS II drawing of the scatterometry lines Pattern 5-5 at wide FOV. (bottom) SEM micrograph of a similar area. 

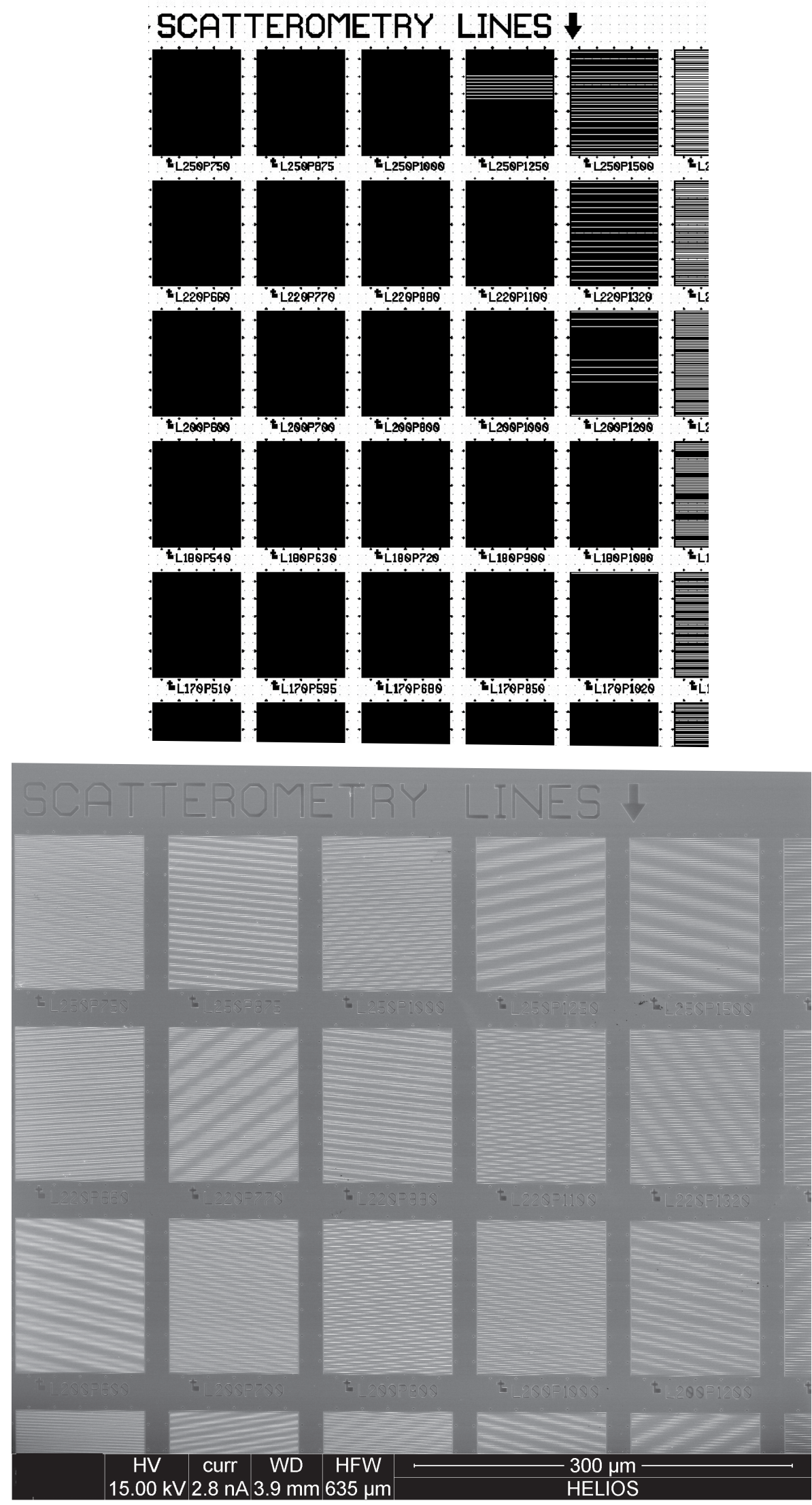

Plate 79 - RM 8820 Section 5; Pattern 5-5 
Plate 80 - Pattern 5-5 - Scatterometry lines (top) GDS II drawing of the scatterometry lines Pattern 5-5 at wide FOV. (bottom) SEM micrograph of a similar area. 

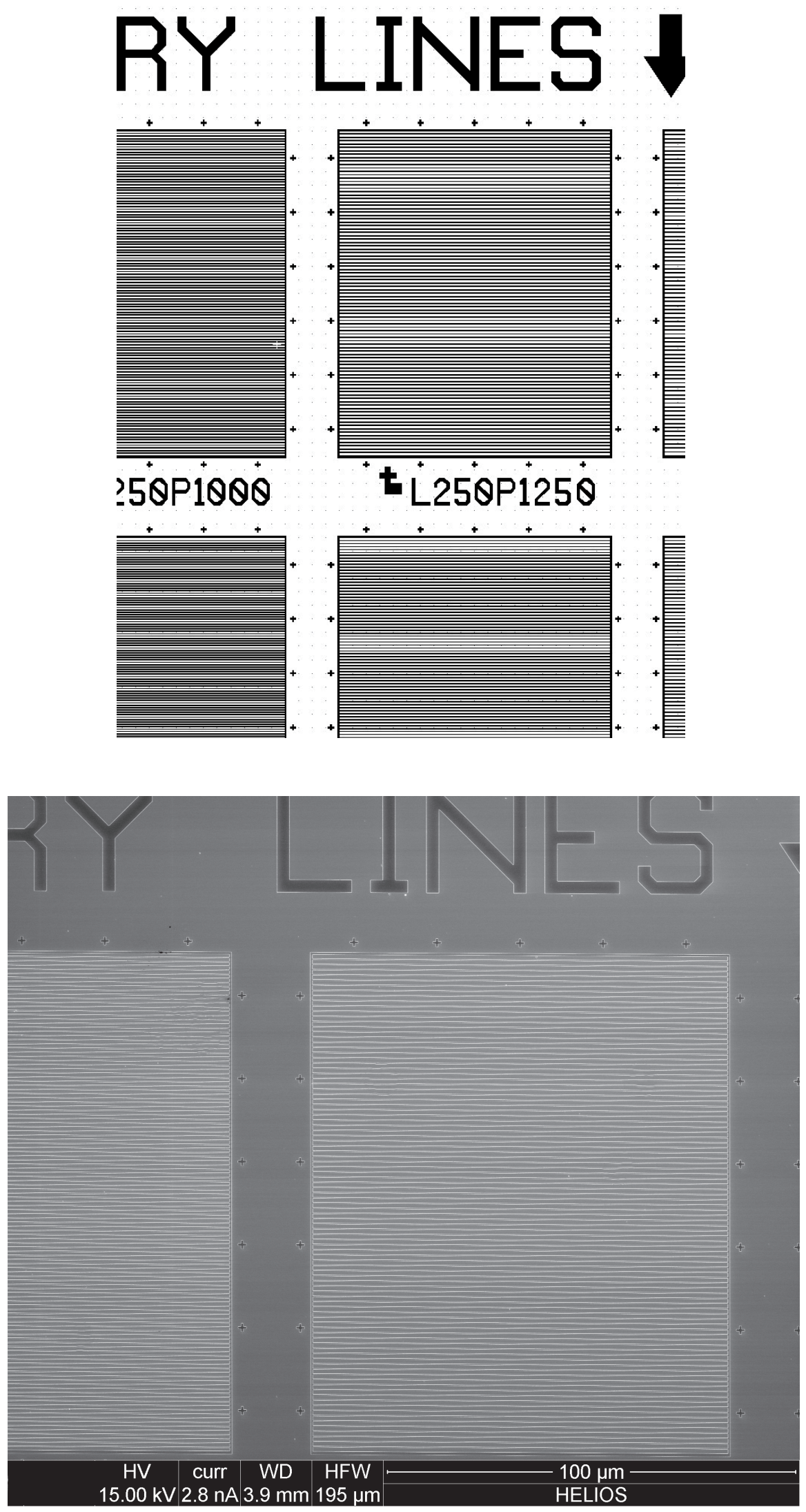

Plate 80 - Pattern 5-5 - Scatterometry lines 
Plate 81 - Patterns 5-6, 5 - 7 and 5 - 8 - Line Edge Roughness. (top) GDS II line drawing of the line edge roughness patterns. (bottom) SEM micrograph of a similar area.

\section{Explanation:}

Line Edge Roughness (LERBEN). The module contains $20 \mu \mathrm{m}$ long lines with various assist features for printing intentional periodic roughness artifacts similar to above. Amplitude: $10 \mathrm{~nm}$ to $100 \mathrm{~nm}$ (in $10 \mathrm{~nm}$ steps), $120 \mathrm{~nm}$, and $150 \mathrm{~nm}$. The periodicity is: 50 to 260 (10 nm steps), 280 to 340 (20 nm steps), 370, 400, 450, 500; the tabs are 1/3 of the periodicity. These additional LER patterns were designed by Benjamin Bunday of SEMATECH. The different amplitudes and periodicities and widths of the carrier lines are shown, with different styles of assist features, and each module is repeated 4 times for giving redundancy in cases of resist shrinkage. 

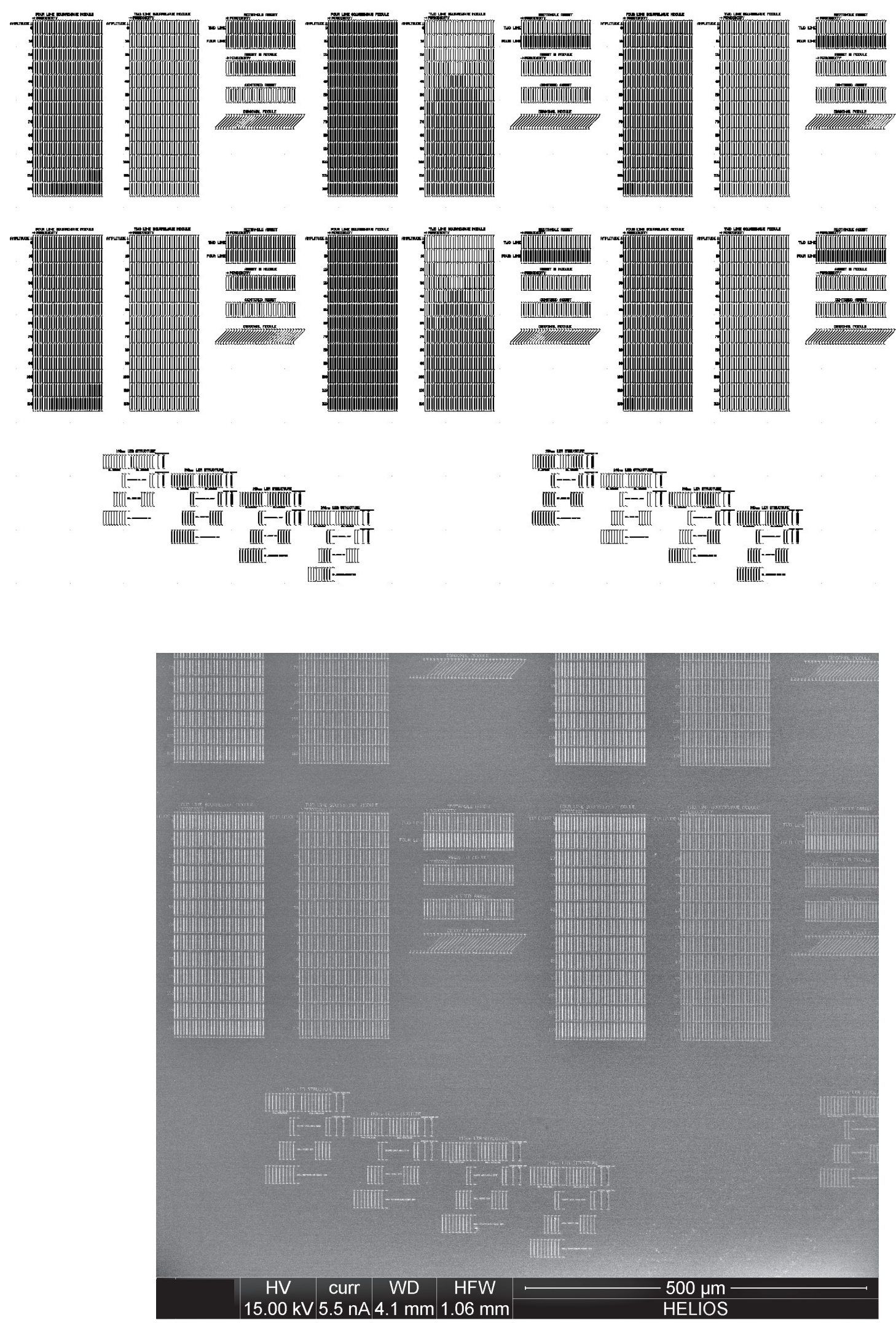

Plate 81 - Patterns 5-6, 5 - 7 and 5 - 8 - Line Edge Roughness. 
Plate 82 - Pattern 5-6 - Line Edge Roughness. (top) GDS II line drawing of the line edge roughness patterns of Pattern 5-6. (bottom) SEM of a similar area of the $140 \mathrm{~nm}$ LER structure. 


\section{0nm LER STRUCTURE}
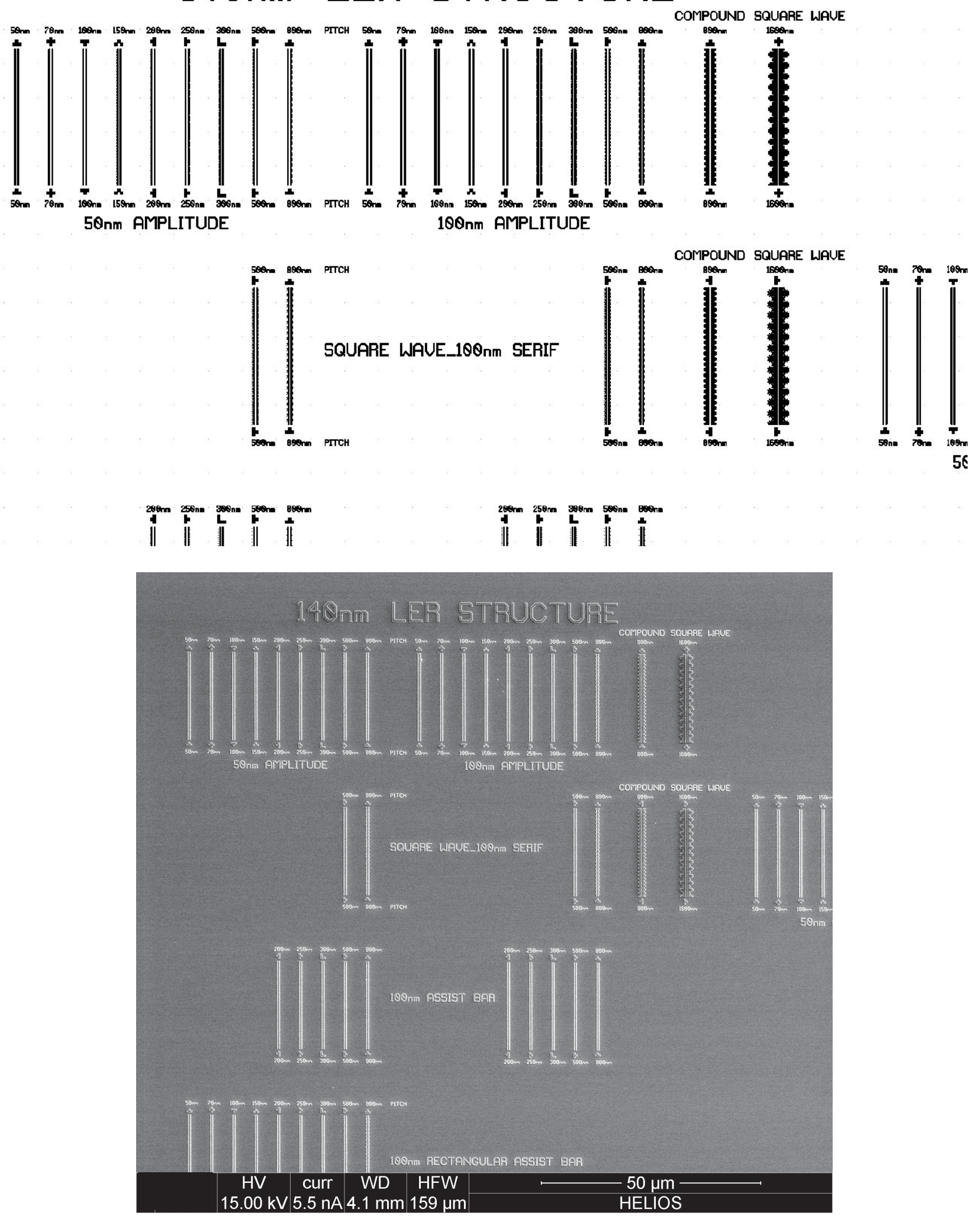

Plate 82 - Pattern 5-6 - Line Edge Roughness 
Plate 83 - Pattern 5-6 - Line Edge Roughness. (top) GDS II line drawing of the line edge roughness patterns of Pattern 5 - 6 showing compound square wave roughness. (bottom) SEM of a similar area of the LER structure. 
COMPOUND SQUARE WAUE

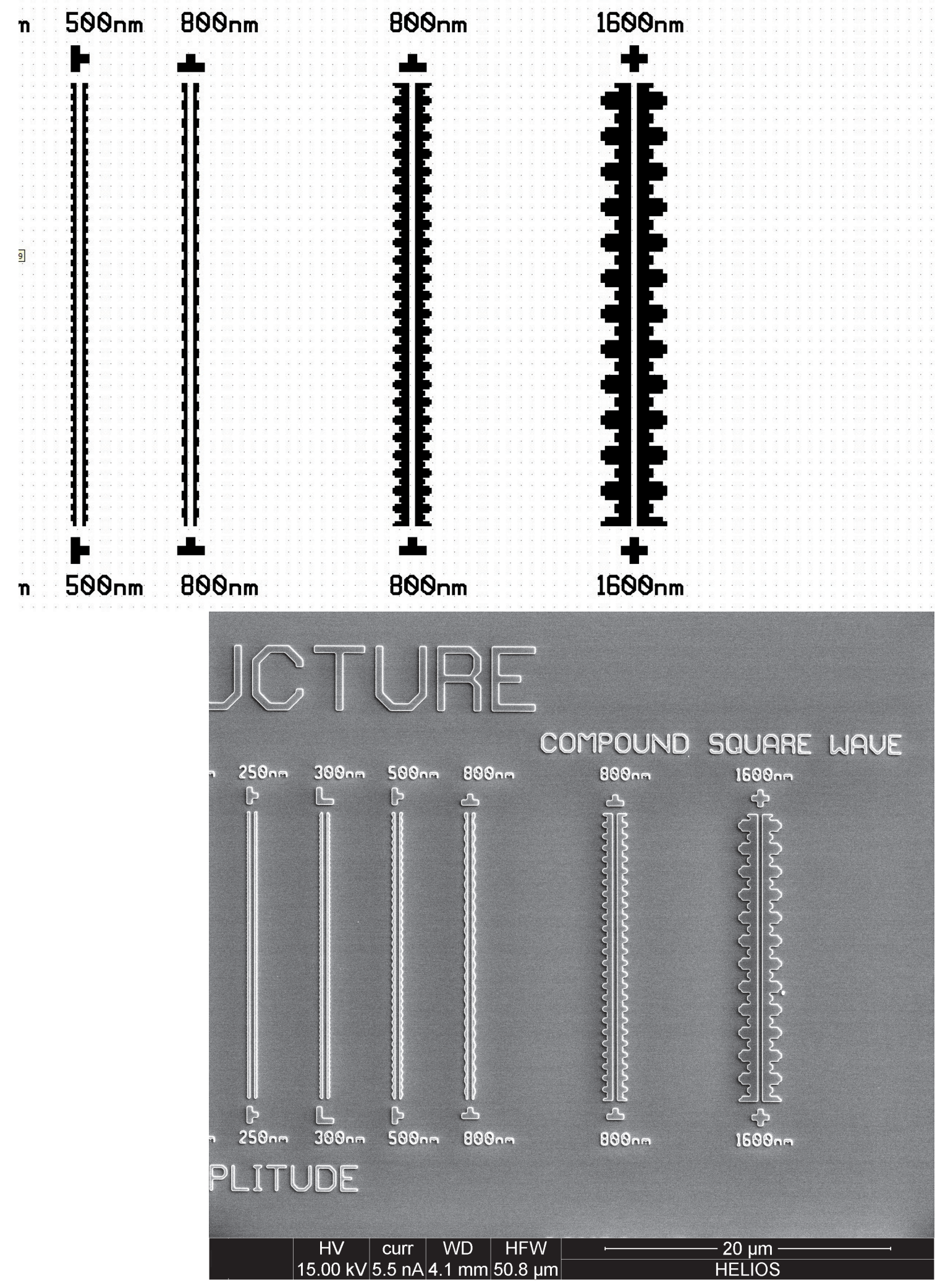

Plate 83 - Pattern 5-6 - Line Edge Roughness. 
Plate 84 - Pattern 5-7 - Line Edge Roughness. (top) GDS II line drawing of the line edge roughness patterns of Pattern 5 - 7 showing the of the four line square wave periodicity. (bottom) SEM of a similar area of the structure. 

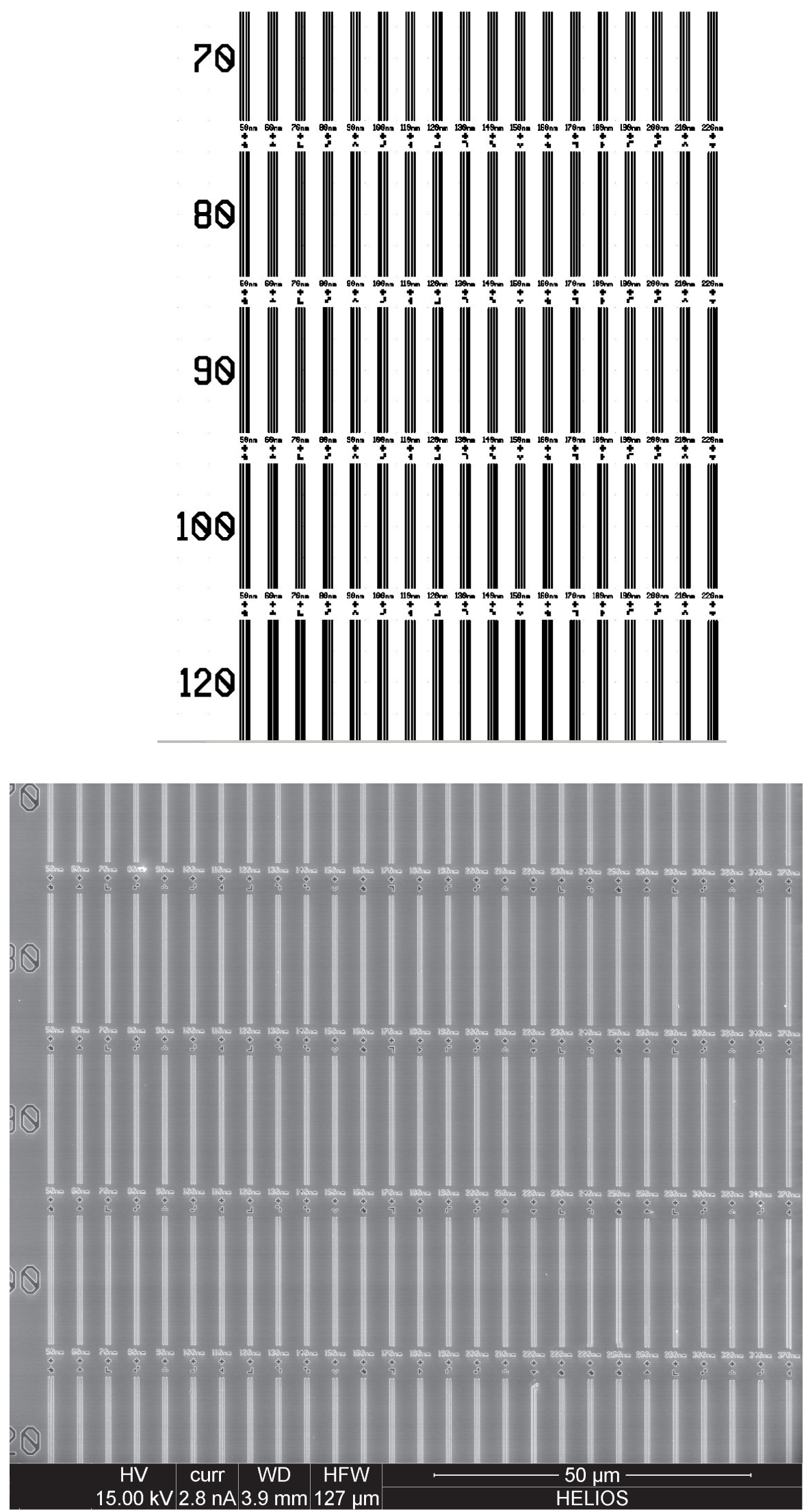

Plate 84 - Pattern 5-7 - Line Edge Roughness 
Plate 85 - Pattern 5-7 - Line Edge Roughness. (top) GDS II line drawing of the line edge roughness patterns of Pattern 5 - 7 showing the centered assist and diagonal modules. (bottom) SEM of a similar area of the structure. 


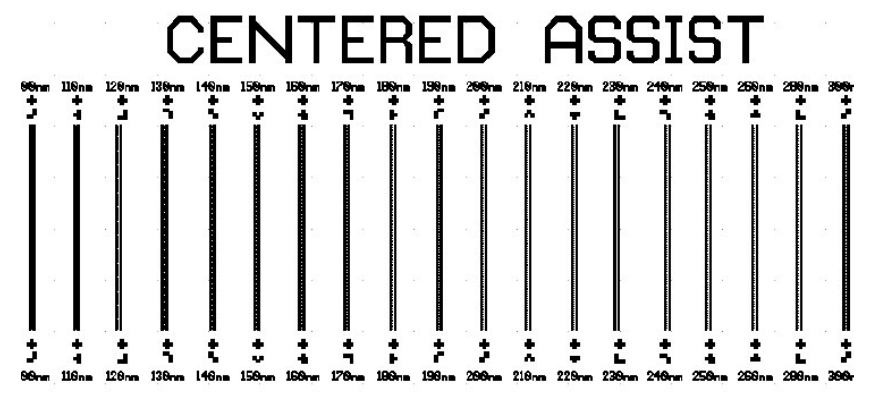

\section{DIAGONAL MODULE}
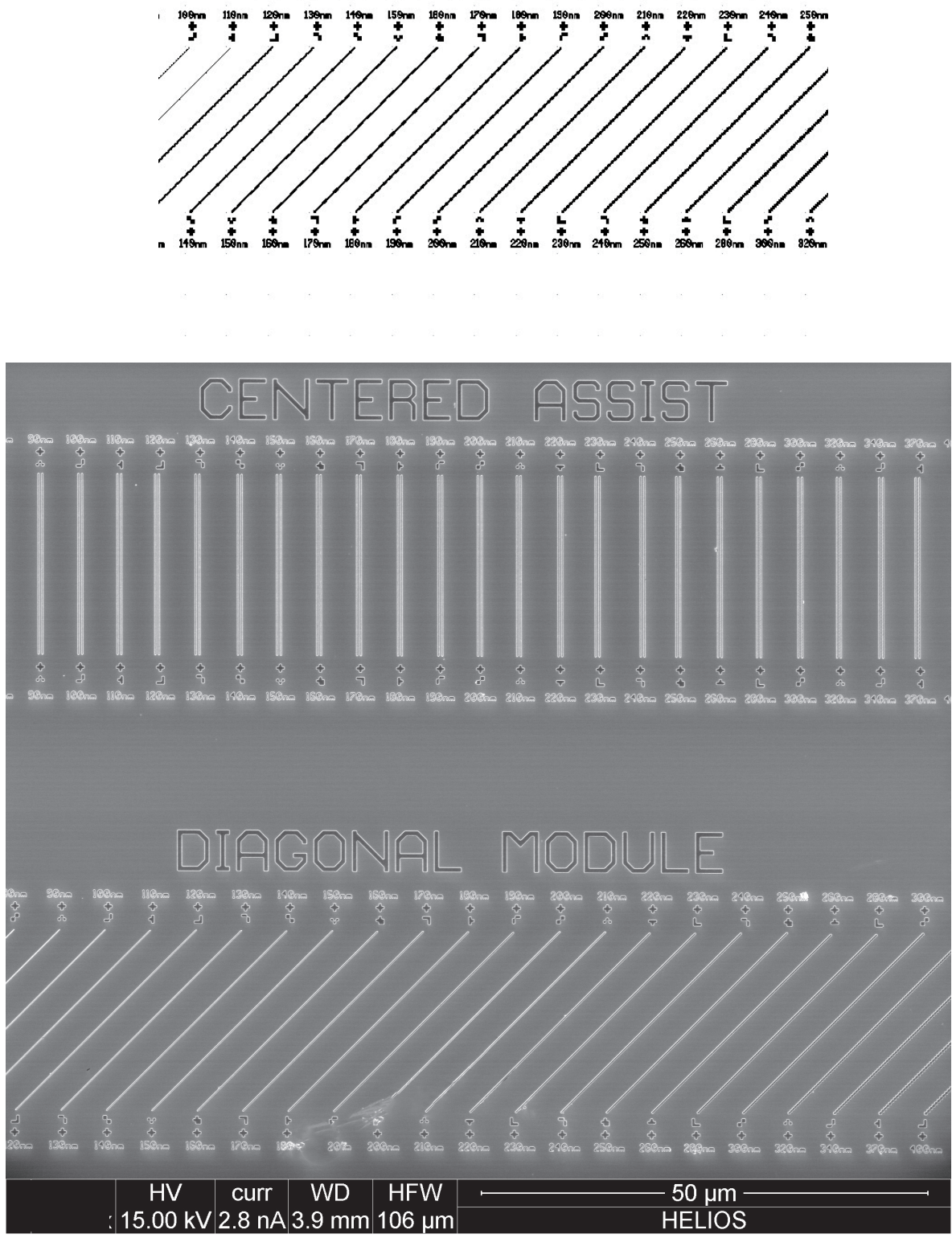

Plate 85 - Pattern 5-7 - Line Edge Roughness 
Plate 86 - Pattern 5-8 - Line Edge Roughness. (top) GDS II line drawing of the line edge roughness at reduced FOV of the four line square wave periodicity. (bottom) SEM micrograph of a similar area. 

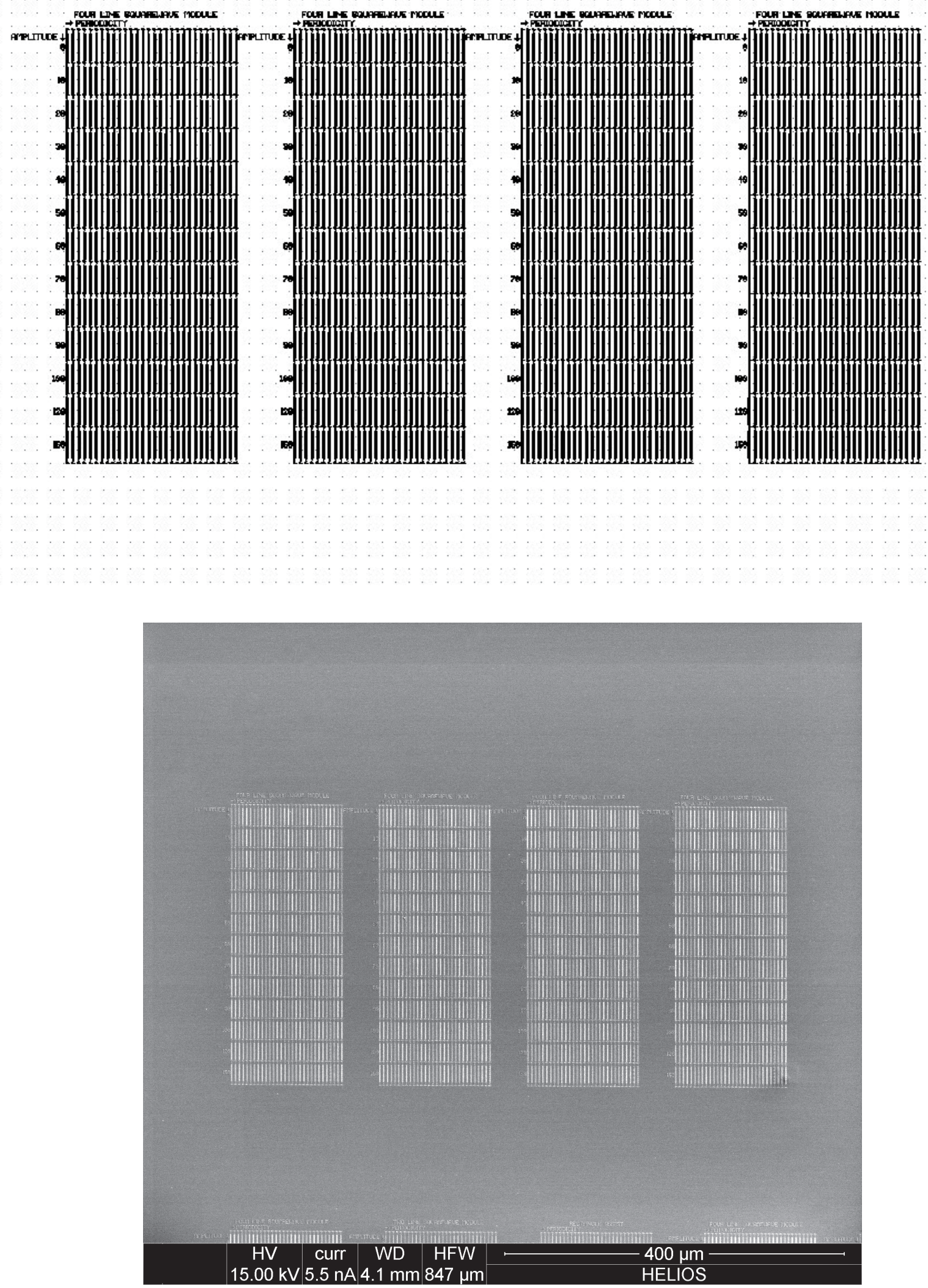

Plate 86 - Pattern 5-8 - Line Edge Roughness 
Plate 87 - Pattern 5-8 - Line Edge Roughness. (top) GDS II line drawing of the line edge roughness at reduced FOV of the four line square wave periodicity. (bottom) SEM micrograph of a similar area. 

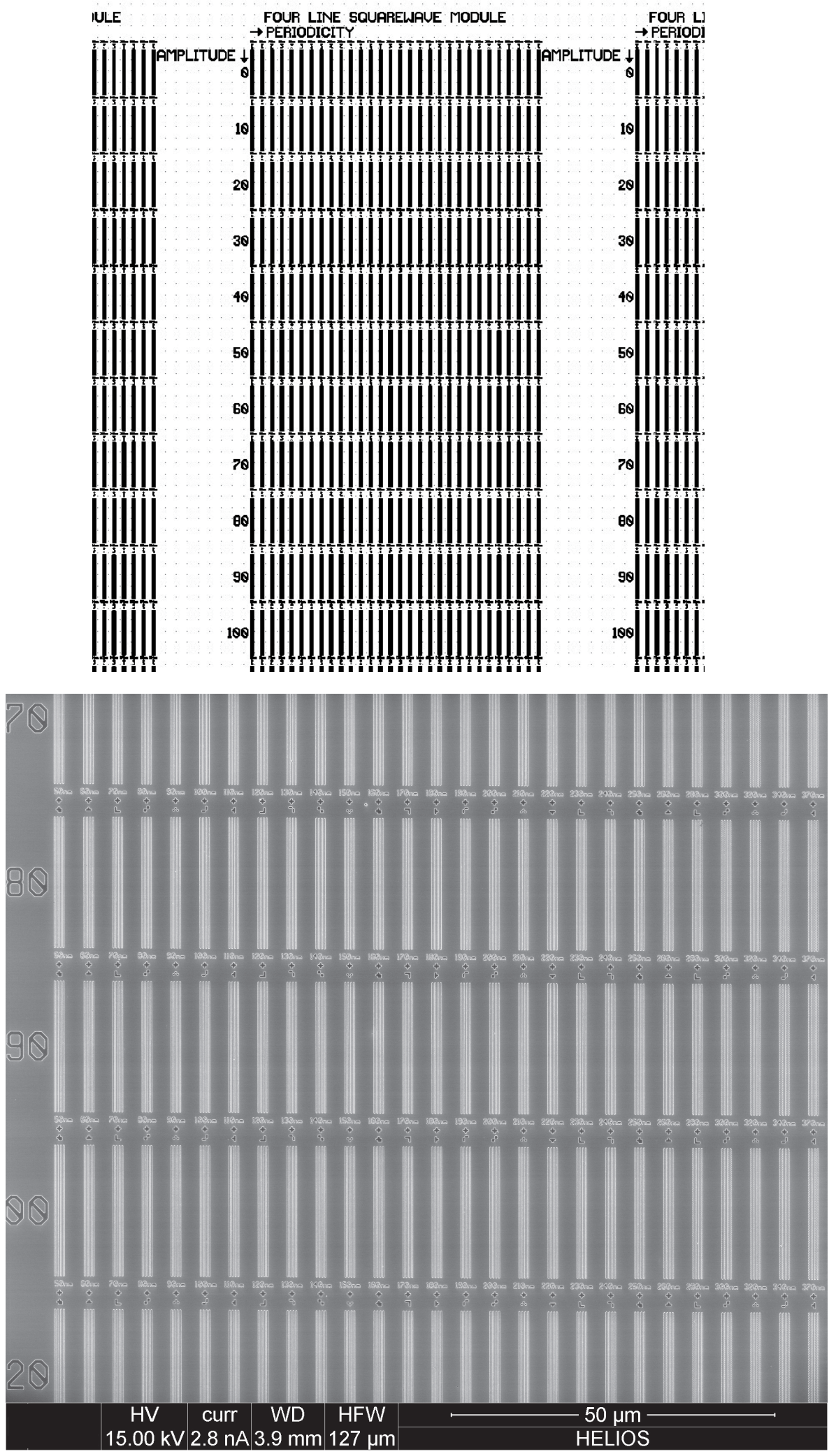

Plate 87 - Pattern 5-8 - Line Edge Roughness 
Plate 88 - Pattern 5 - 8 - Line Edge Roughness. (top) GDS II line drawing of the line edge roughness at reduced FOV of the four line square wave periodicity. (bottom) SEM micrograph of a similar area. 


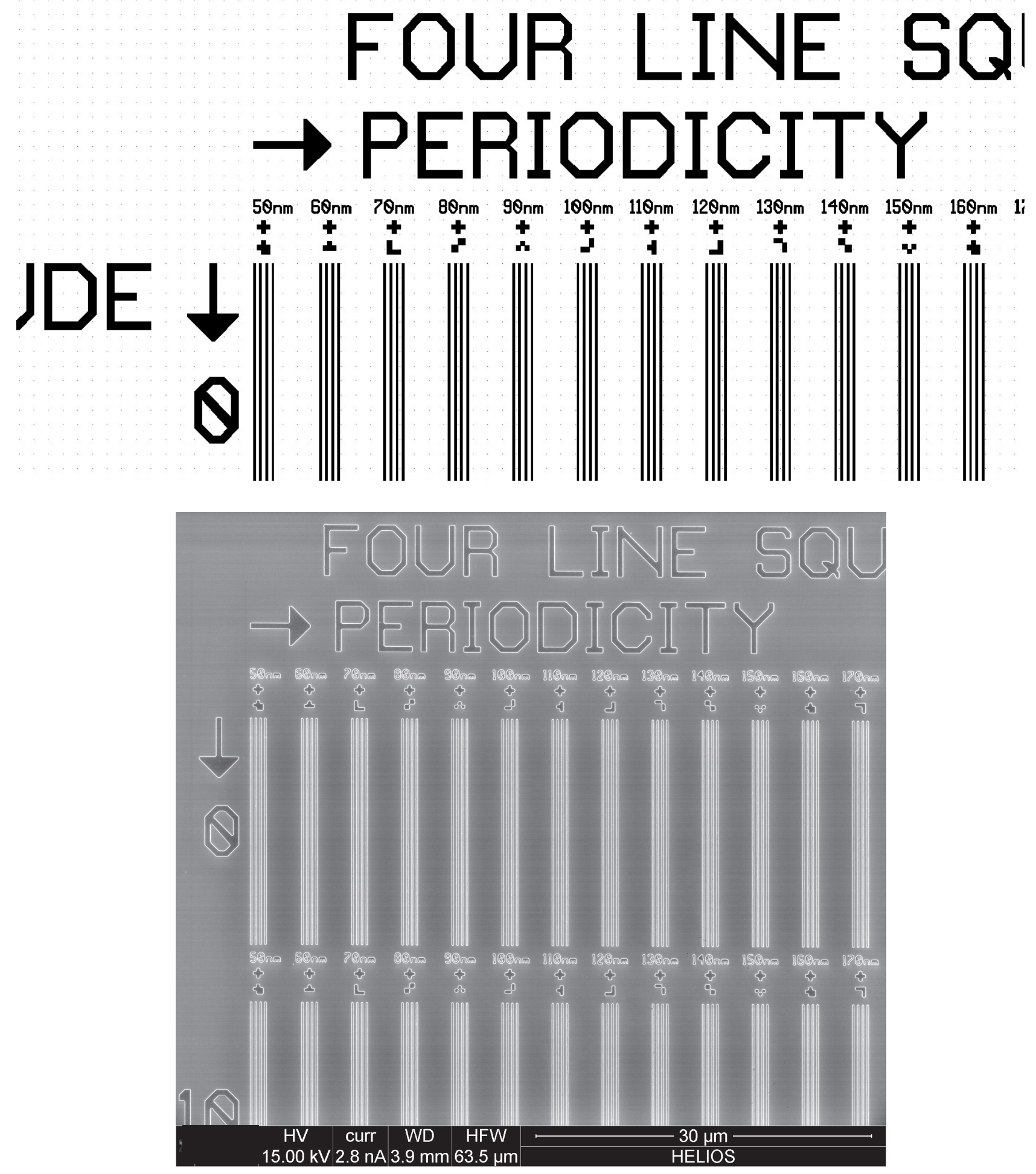

Plate 88 - Pattern 5 - 8 - Line Edge Roughness. 
Plate 89 - Pattern 5 - 9 - SEM Pattern Recognition. (top) GDS II line drawing of the SEM Pattern Recognition module at a wide FOV. (bottom) SEM micrograph of a similar area.

\section{Explanation:}

SEM Pattern Recognition. This module is an array of: 1) pattern recognition symbols of various sizes with and without dummy fill. The symbol sizes are varied and are filled with $180 \mathrm{~nm}$ line/spaces and in vertical and horizontal directions, others are solid and some are filled with $180 \mathrm{~nm}$ contact holes. 


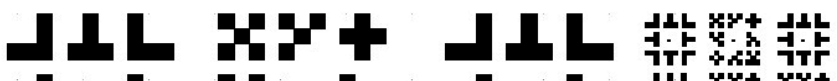
Hry

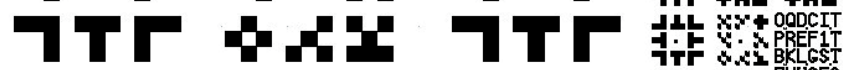

J1 x+

fry $y$ y

TTr A $A x+A$

IL XP+OODCIT

A r $\boldsymbol{y}$ PREF $1 \mathrm{~T}$

TTr

$+A x_{0 H A G G}$

$\mathrm{Ax} \mathrm{Ax}$

XX $\mathrm{xH}$

\author{
$=$
}

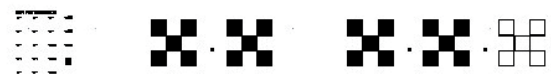

SEM PATTERN REC

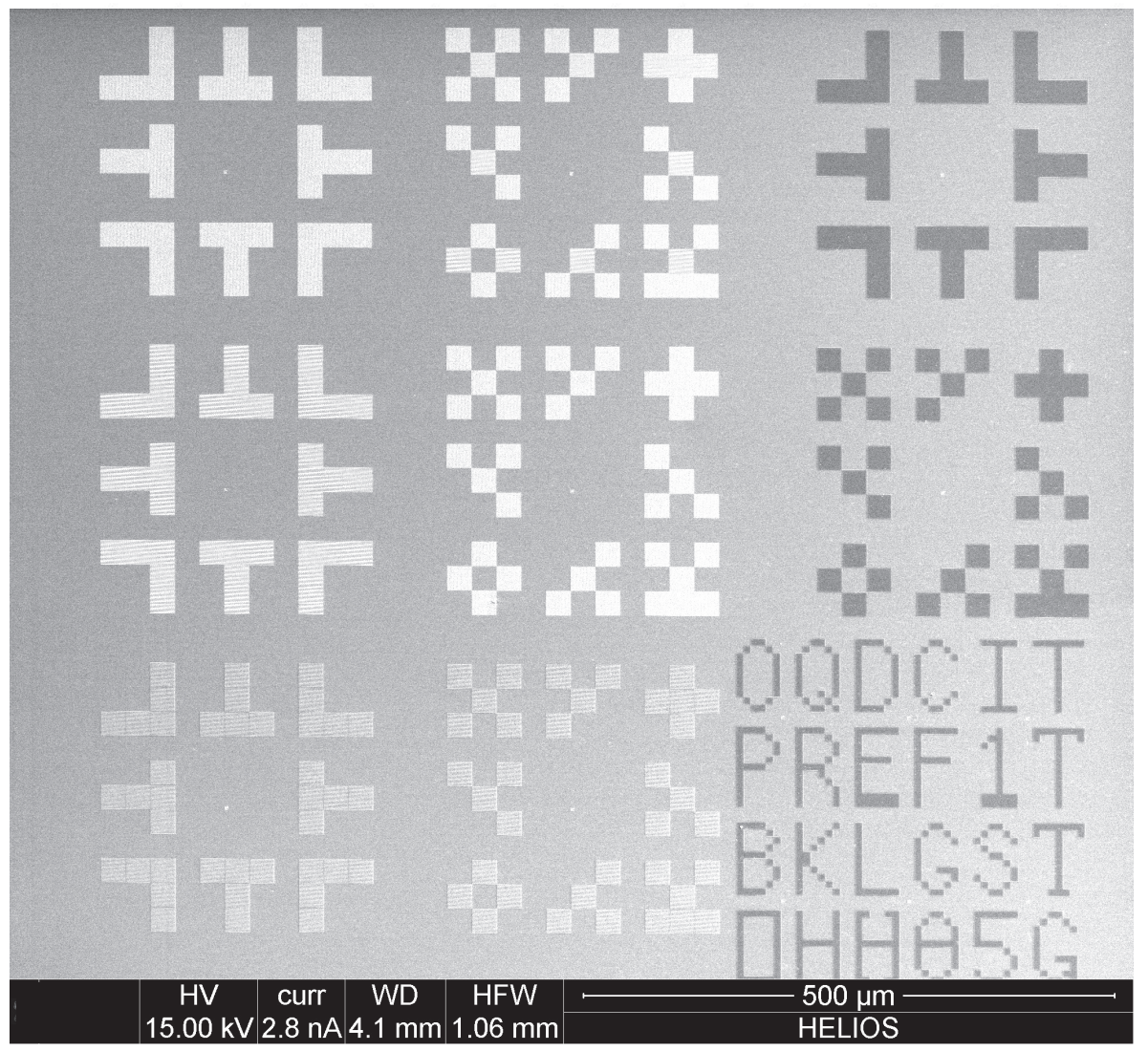

Plate 89 - Pattern 5 - 9 - SEM Pattern Recognition 
Plate 90 - Pattern 5 - 9 - SEM Pattern Recognition. (top) GDS II line drawing of the SEM Pattern Recognition module at a reduced FOV. (bottom) SEM micrograph of a similar area. 


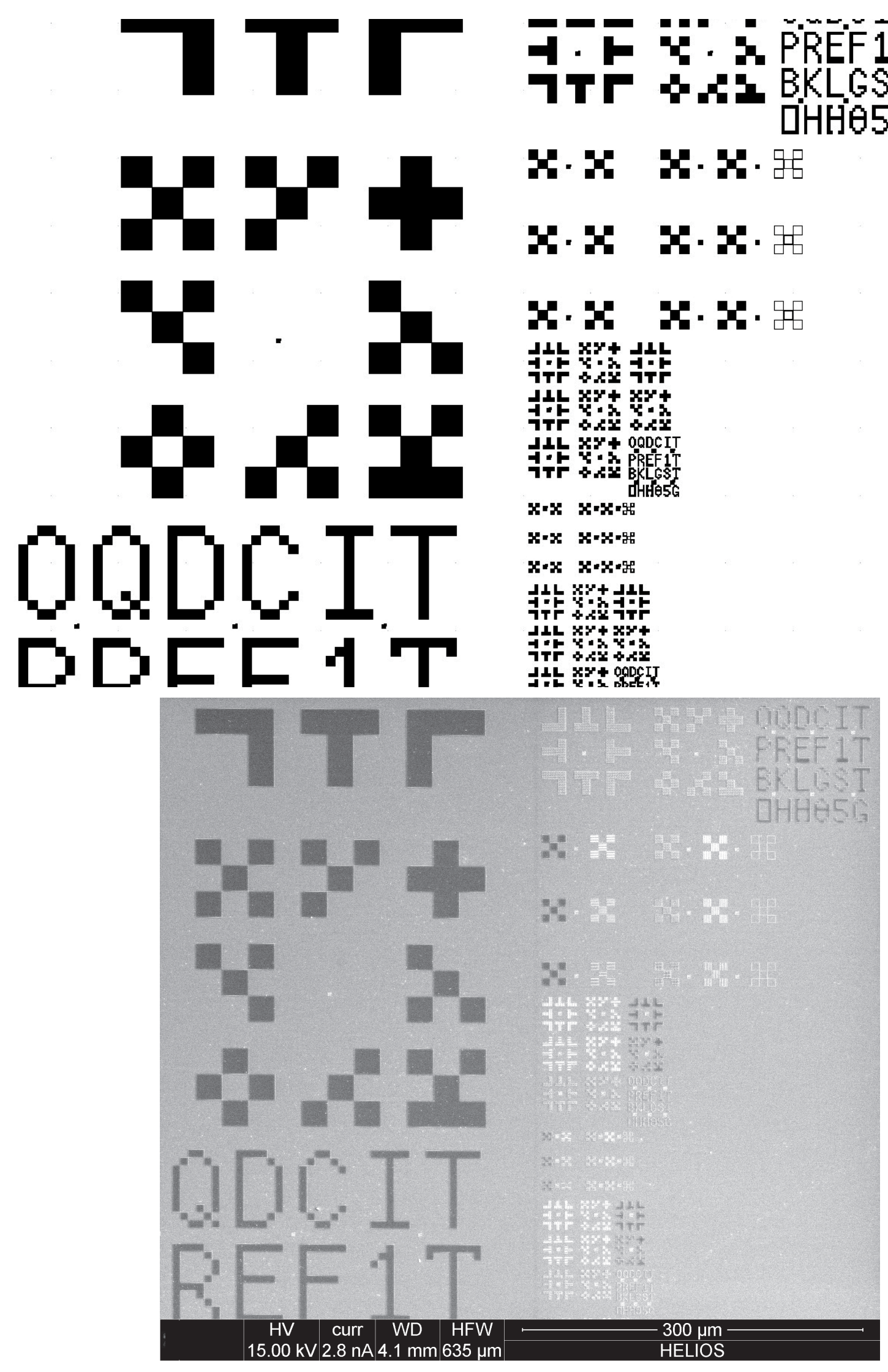

Plate 90 - Pattern 5 - 9 - SEM Pattern Recognition 
Plate 91 - Pattern 5 - 9 - SEM Pattern Recognition. (top) SEM Micrograph of the SEM Pattern Recognition module at an HFW of (top) $159 \mu \mathrm{m}$ and (bottom) $84.7 \mu \mathrm{m}$. 


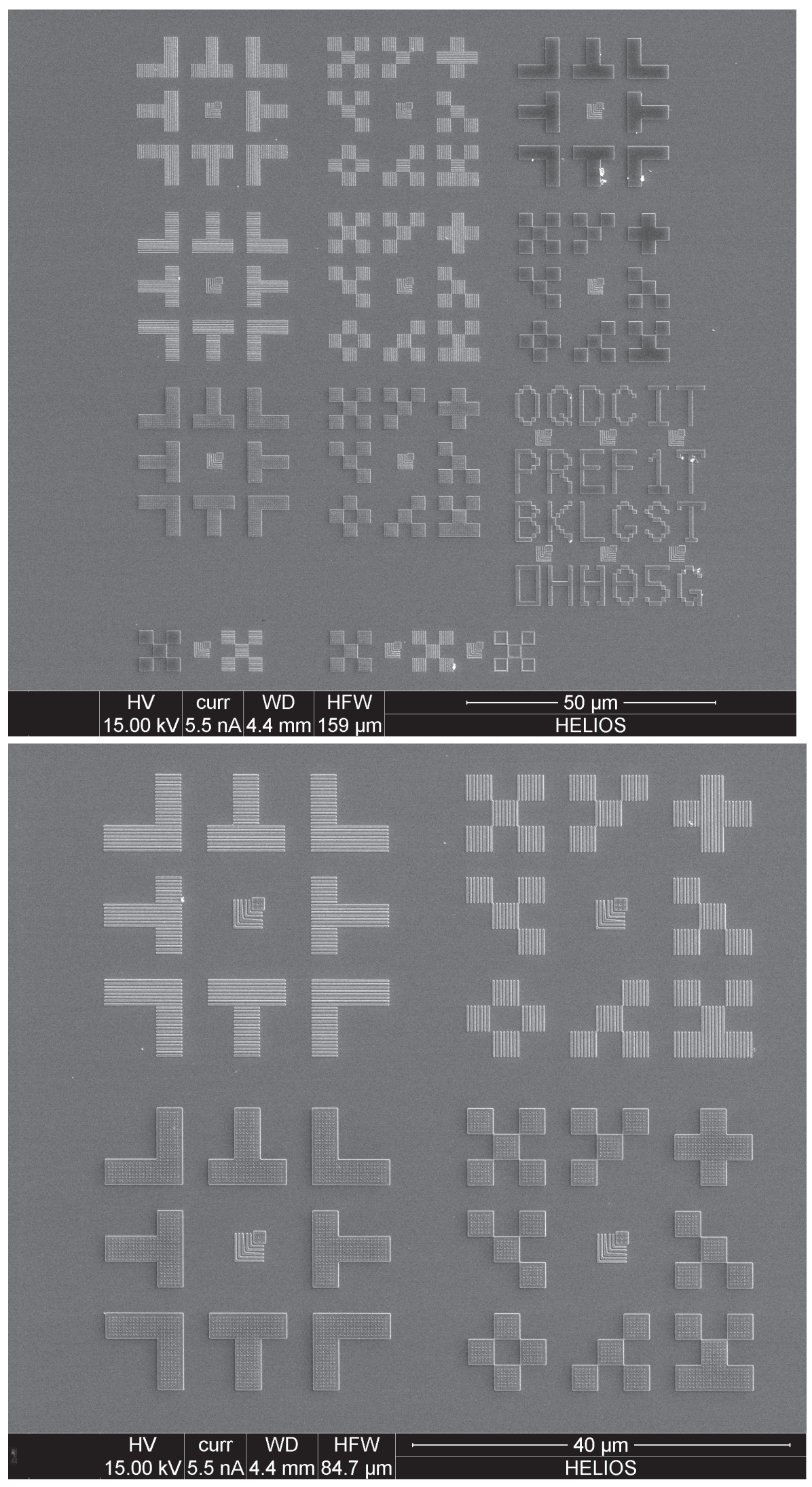

Plate 91 - Pattern 5 - 9 - SEM Pattern Recognition 
Plate 92 - Pattern 5-10 - Line End. (top) GDS II line drawing of the line end pullback and line end pull back links patterns a wide FOV. (bottom) SEM micrograph of a similar area.

\section{Explanation:}

LineEnd. These modules are designed to show effects of line end shortening and enable reliable measurement of this unavoidable consequence of sub-wavelength lithography. There are two types of line end shortening structures: 1) horizontal/vertical block pattern, 2) horizontal link pattern. OPC is added to the last cell in both types as indicated; $15 \mathrm{~nm}$ is added to each side on the block pattern and $10 \mathrm{~nm}$ is added to each side of the link pattern. Block size/space: $150 \mathrm{~nm}$ width $150 \mathrm{~nm}$ space, $160 \mathrm{~nm}$ width $150 \mathrm{~nm}$ space, $150 \mathrm{~nm}$ width $200 \mathrm{~nm}$ space, $160 \mathrm{~nm}$ width $200 \mathrm{~nm}$ space $150 \mathrm{~nm}$ width $200 \mathrm{~nm}$ space with OPC. These are in gratings large enough to be used with OCD.

LineEnd shortening. There are two types of line end shortening structures: 1) horizontal/vertical block pattern and 2) horizontal link pattern. OPC is added to the last cell in both types as indicated; 15 $\mathrm{nm}$ is added to each side on the block pattern and $10 \mathrm{~nm}$ is added to each side of the link pattern. Block size/space: $150 \mathrm{~nm}$ width $150 \mathrm{~nm}$ space, $160 \mathrm{~nm}$ width $150 \mathrm{~nm}$ space, $150 \mathrm{~nm}$ linewidth $200 \mathrm{~nm}$ space, $160 \mathrm{~nm}$ width $200 \mathrm{~nm}$ space $150 \mathrm{~nm}$ width $200 \mathrm{~nm}$ space with OPC. 


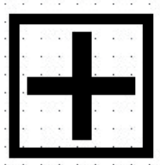

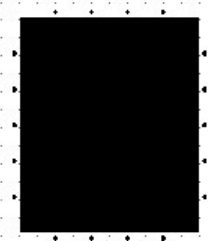

IGONMLINÉ L5ONMGAP

LINE END PULLBACK

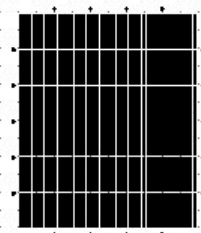

16ONMLINEE

ISONMGAP

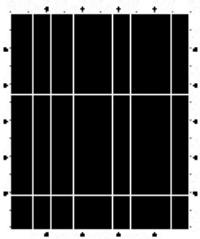

15ONMLINE

15ONMGAP

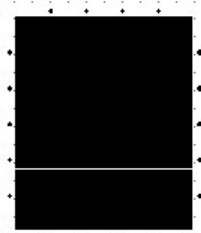

IGONMLINE 2OONMGAP

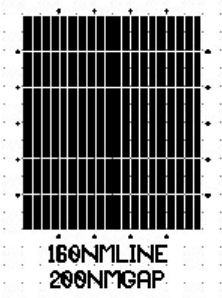

PULLBACK LINKS

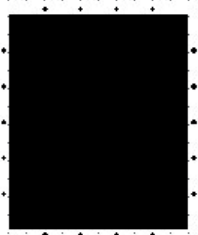

15ONMI INE 2OONMGAP
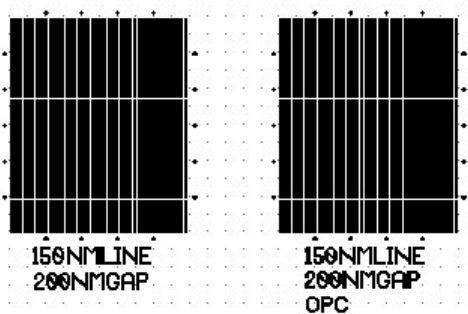

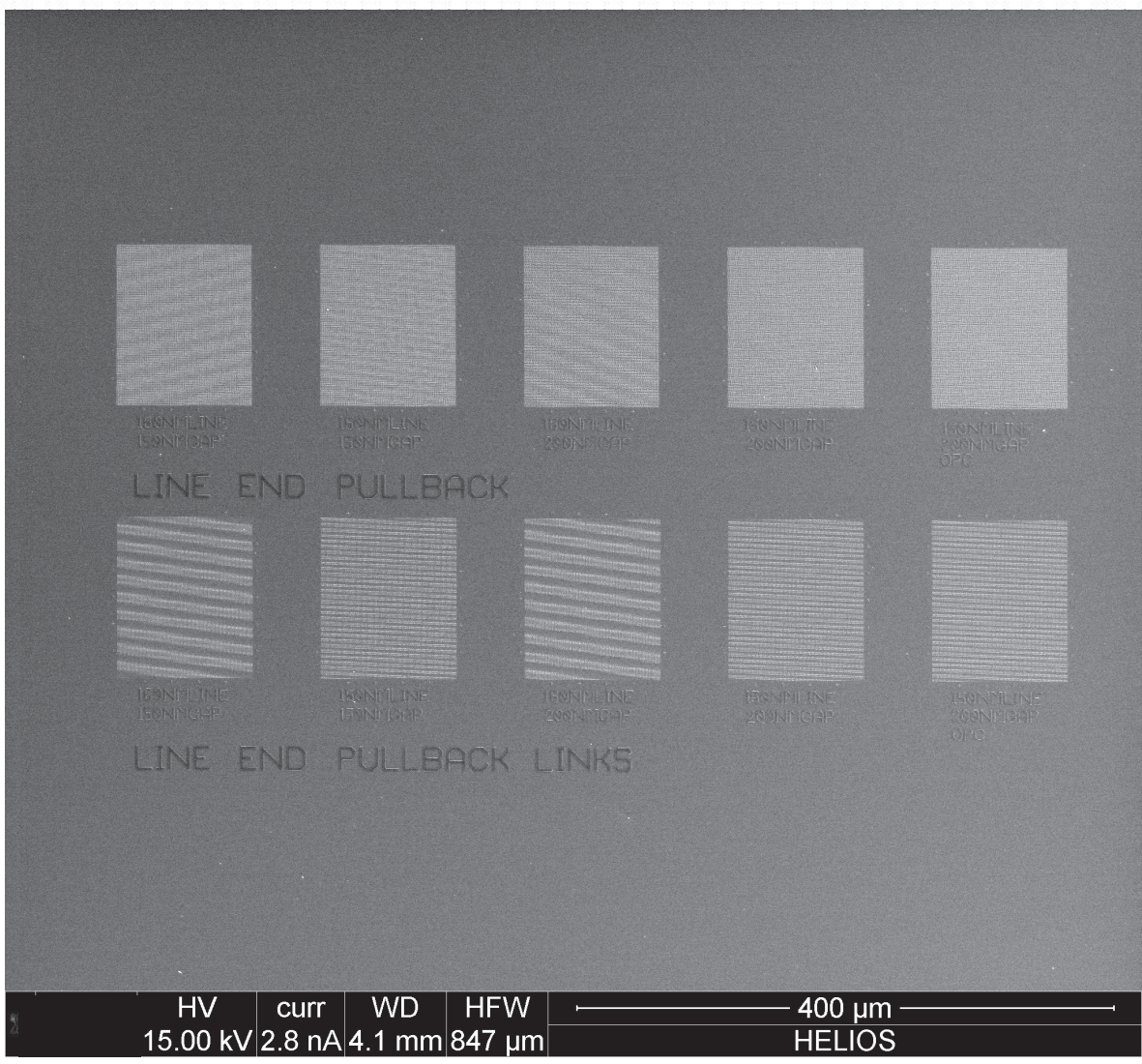

Plate 92 - Pattern 5-10 - Line End 
Plate 93. Pattern 5-10 - Line End Pullback. (top) GDS II line drawing of the line end pullback and line end pull back links patterns a wide FOV. (bottom) SEM micrograph of a similar area. 


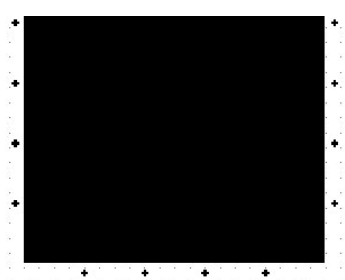

16ONMLINE 15ONMGAP

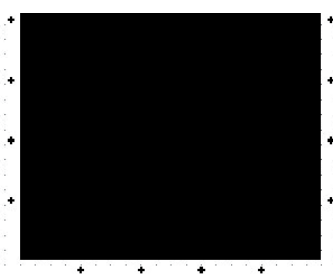

150NMLINE 15ONMGAP

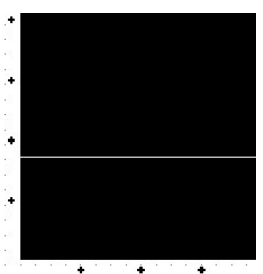

16ONMLINE 2OQNMGAP

\section{LINE END PULLBACK}
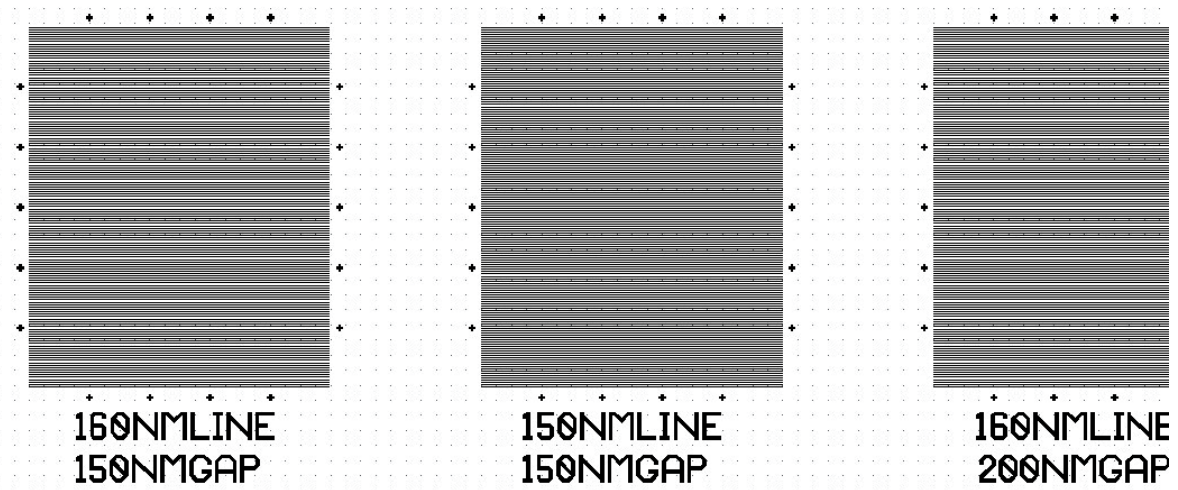

LINE END PULLBACK LINKS

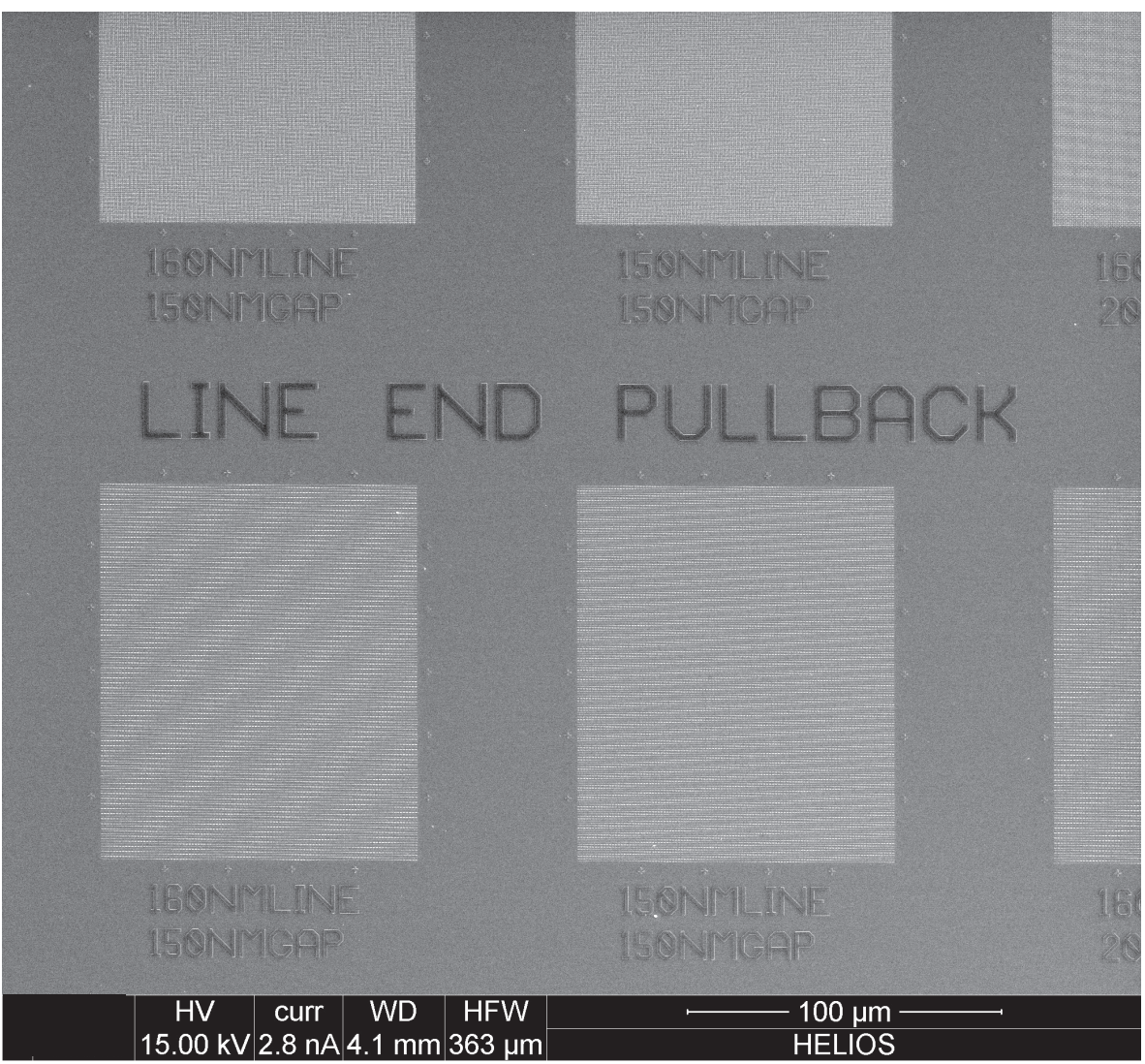

Plate 93. Pattern 5-10 - Line End Pullback 
Plate 94 - Pattern 5 - 10 - Line End Pullback. SEM Micrographs of the $150 \mathrm{~nm}$ (top) and $160 \mathrm{~nm}$ (bottom) lines. 

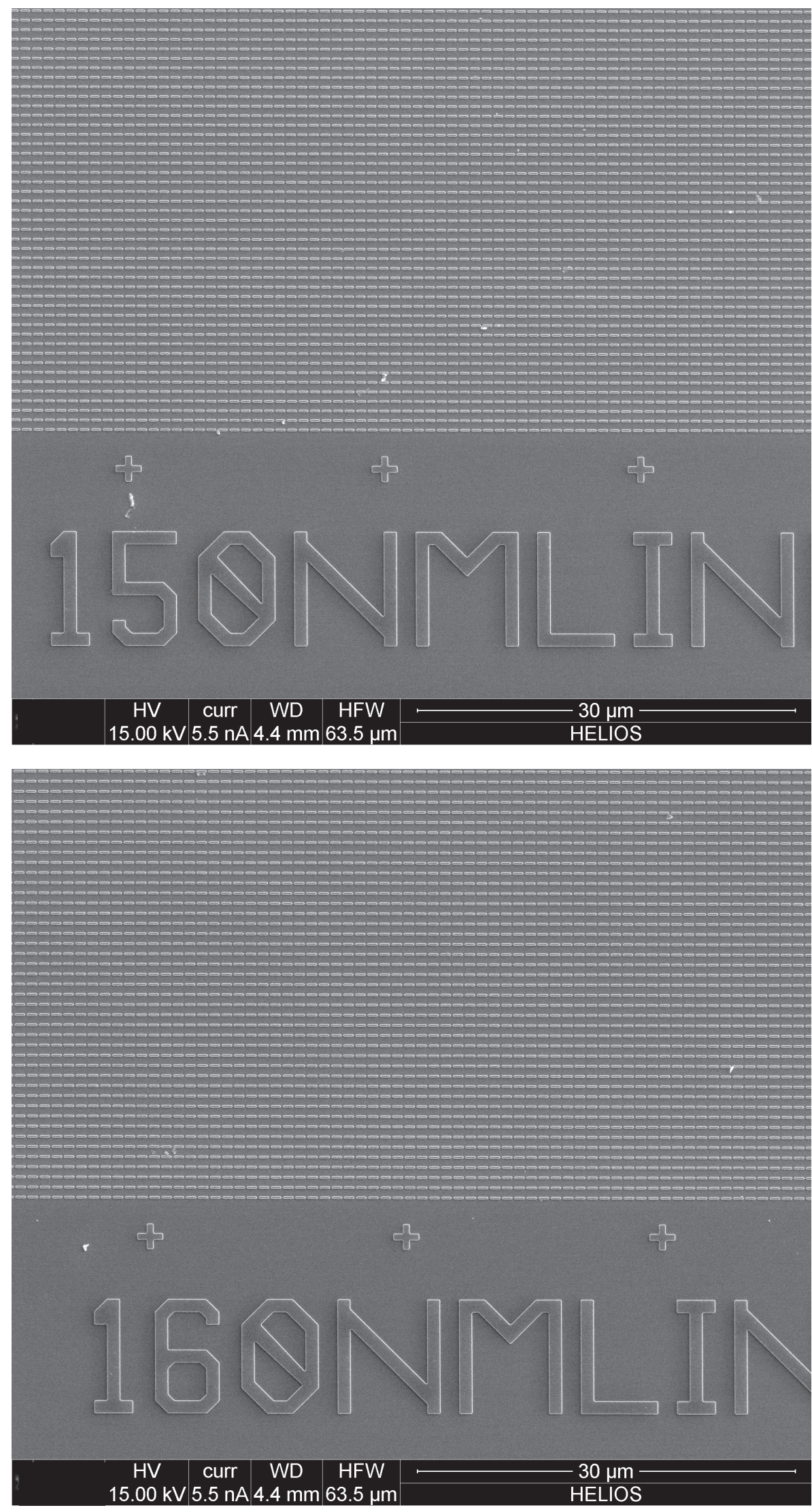

Plate 94 - Pattern 5 - 10 - Line End Pullback 
Page intentionally left blank 
SECTION 6 
Plate 95. Section 6-0 Overall. (top) GDS II line drawing of the entire RM 8820 pattern highlighting Section 6 with the 9 additional major sub-patterns numerically identified. (bottom) GDS II detail drawing of section 6 showing the 9 additional major sub-patterns included in this section. Pattern 6-1 is also seen in the center of this module. 

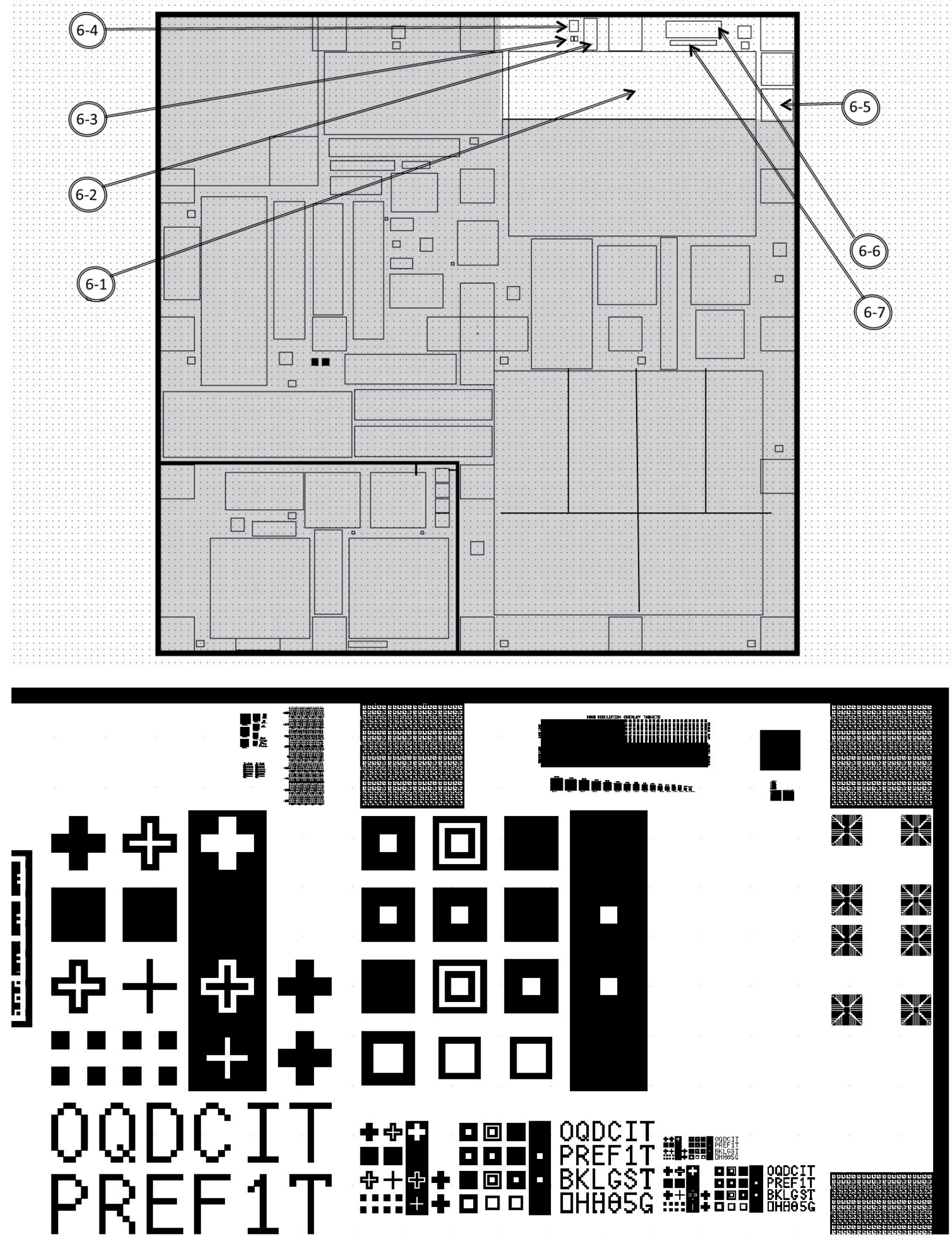

Plate 95. Section 6-0 Overall 
Plate 96 - Patterns 6-2, 6-3 and 6-4. Wide FOV of GDS II line drawing of Patterns 6-2 (SEM overlay), 6-3 (scatterometry) and 6-4 (array target) at a wide FOV. (bottom) SEM micrograph of a similar area. 

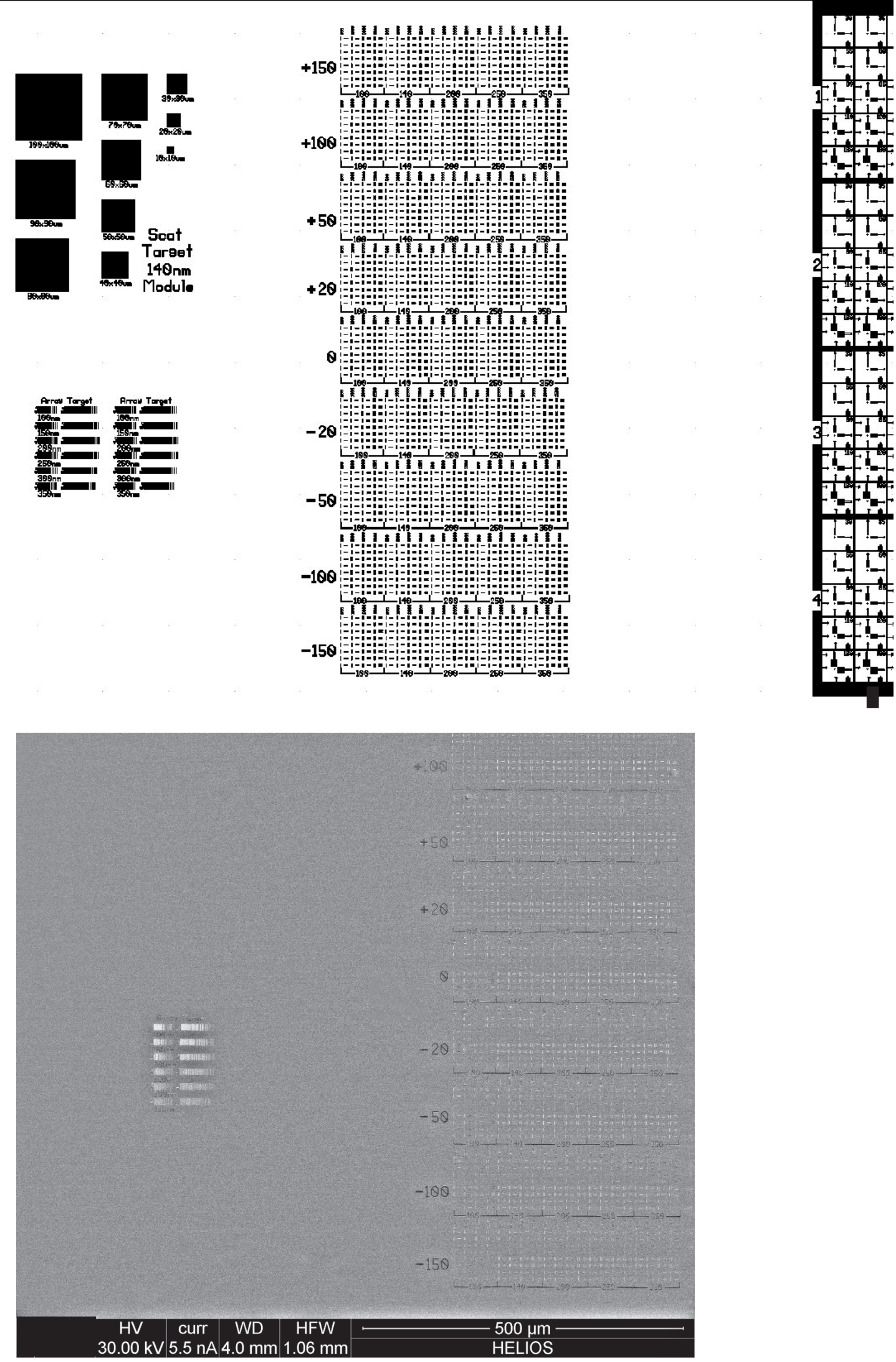

Plate 96 - Patterns 6-2, 6-3 and 6-4 
Plate 97 - Pattern 6-2 - SEM Overlay. GDS II drawing of Pattern 6-2 at a smaller FOV showing some of the detail of the fine patterns. There are several line groups with different design rules and different built-in offsets of the center line for testing SEM overlay measurement capabilities.

\section{Explanation:}

There are several line groups with different design rules and different built-in offsets of the center line for testing SEM overlay measurement capabilities.

SEM Overlay. The SEM overlay structures were designed with multiple groups of three lines. The groups are placed both horizontally and vertically in the module. CDs are $100 \mathrm{~nm}, 140 \mathrm{~nm}, 200 \mathrm{~nm}$, $250 \mathrm{~nm}, 300 \mathrm{~nm}$. The inner line is offset by the following steps in the $\mathrm{X}$ and $\mathrm{Y}$ direction: $-150 \mathrm{~nm}$, $-100 \mathrm{~nm},-50 \mathrm{~nm},-20 \mathrm{~nm}, 0 \mathrm{~nm}, 20 \mathrm{~nm}, 50 \mathrm{~nm}, 100 \mathrm{~nm}, 150 \mathrm{~nm}$. All polarities were used for the SEM Overlay structures. 

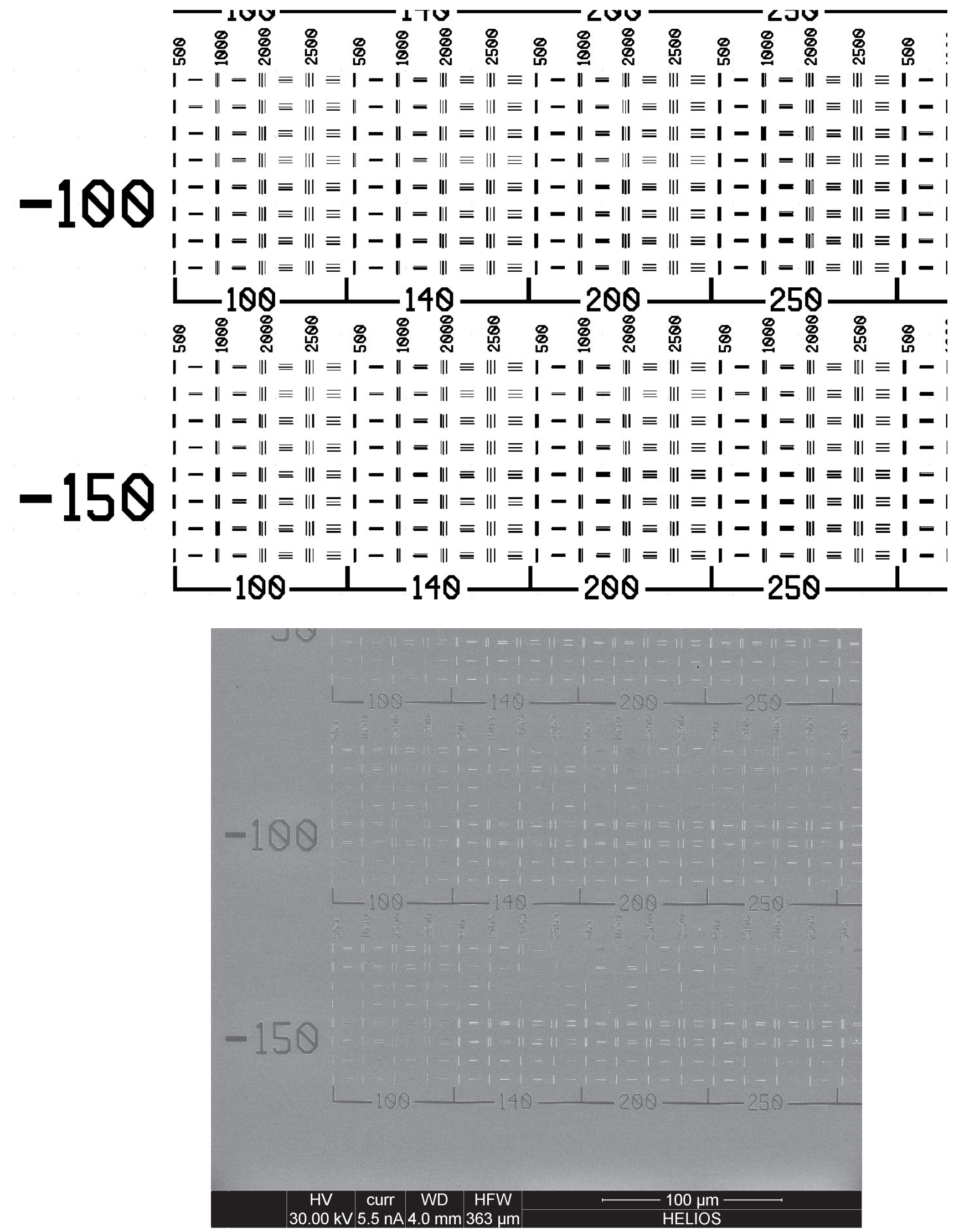

Plate 97 - Pattern 6-2 - SEM Overlay. 
Plate 98 - Pattern 6-3 - Array Target. (top) GDS II line drawing of the array target module at a wide FOV. (bottom) SEM micrograph of a similar area.

\section{Explanation:}

Array Target. The array target is composed of two types of modules. Module 1 has four lines with three spaces and the Module 2 has 10 lines and 9 spaces. The critical dimensions (CDs) are $100 \mathrm{~nm}$ $150 \mathrm{~nm}, 250 \mathrm{~nm}, 300 \mathrm{~nm}$ and $350 \mathrm{~nm}$. The pitch starts at 1:1 and then is spaced as follows: $100 \mathrm{~nm}$, $150 \mathrm{~nm}, 200 \mathrm{~nm}, 250 \mathrm{~nm}, 300 \mathrm{~nm}, 350 \mathrm{~nm}, 400 \mathrm{~nm}, 450 \mathrm{~nm}, 500 \mathrm{~nm}, 1000 \mathrm{~nm}, 2000 \mathrm{~nm}$. Therefore, the $200 \mathrm{~nm}$ line would start as a 1:1 and increase in spacing following the above spacing requirements. 

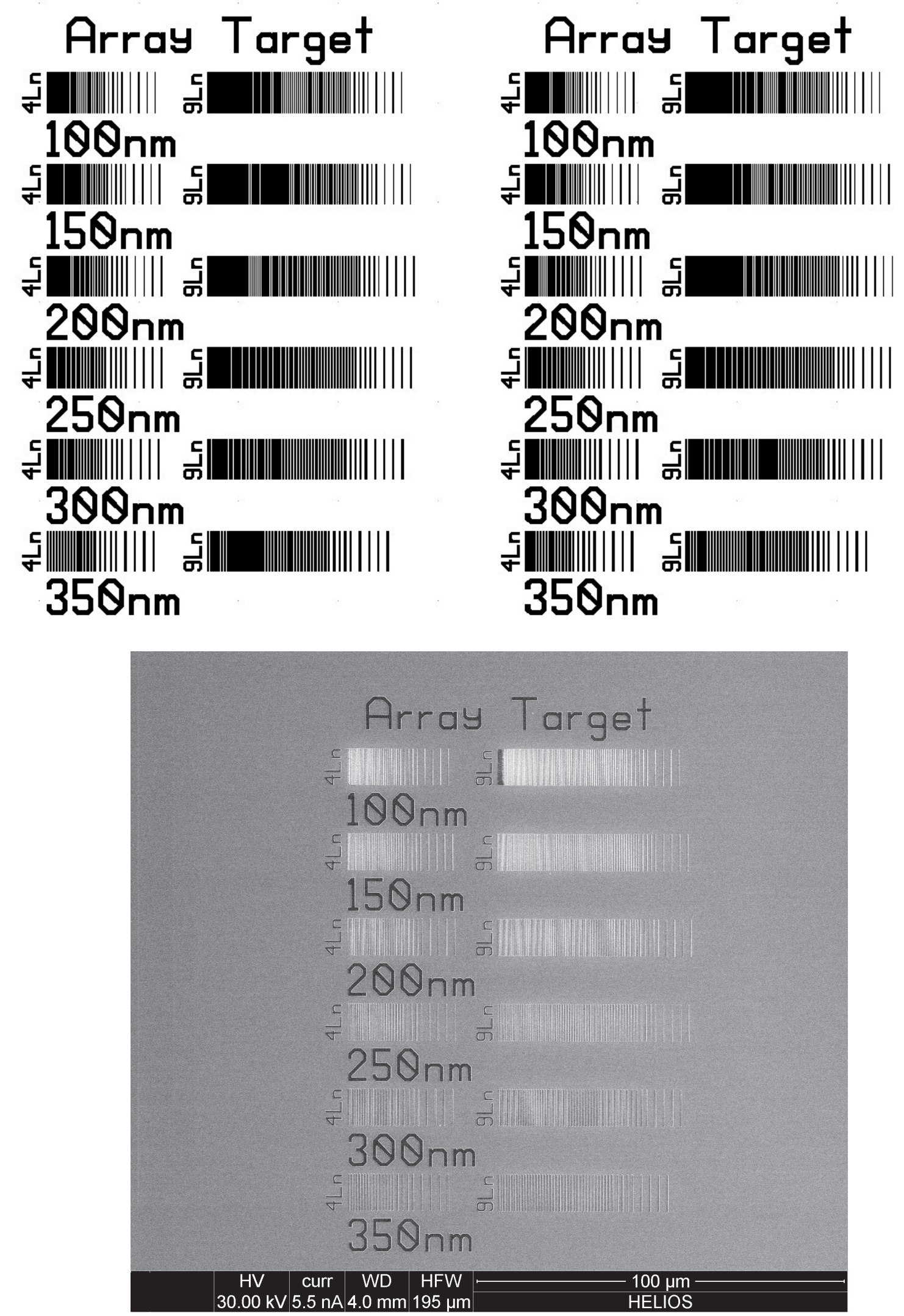

Plate 98. Pattern 6-3 - Array Target 
Plate 99 - Pattern 6-3 - Array Target. (top) GDS II line drawing of the array target module at a wide FOV. (bottom) SEM micrograph of a similar area. 

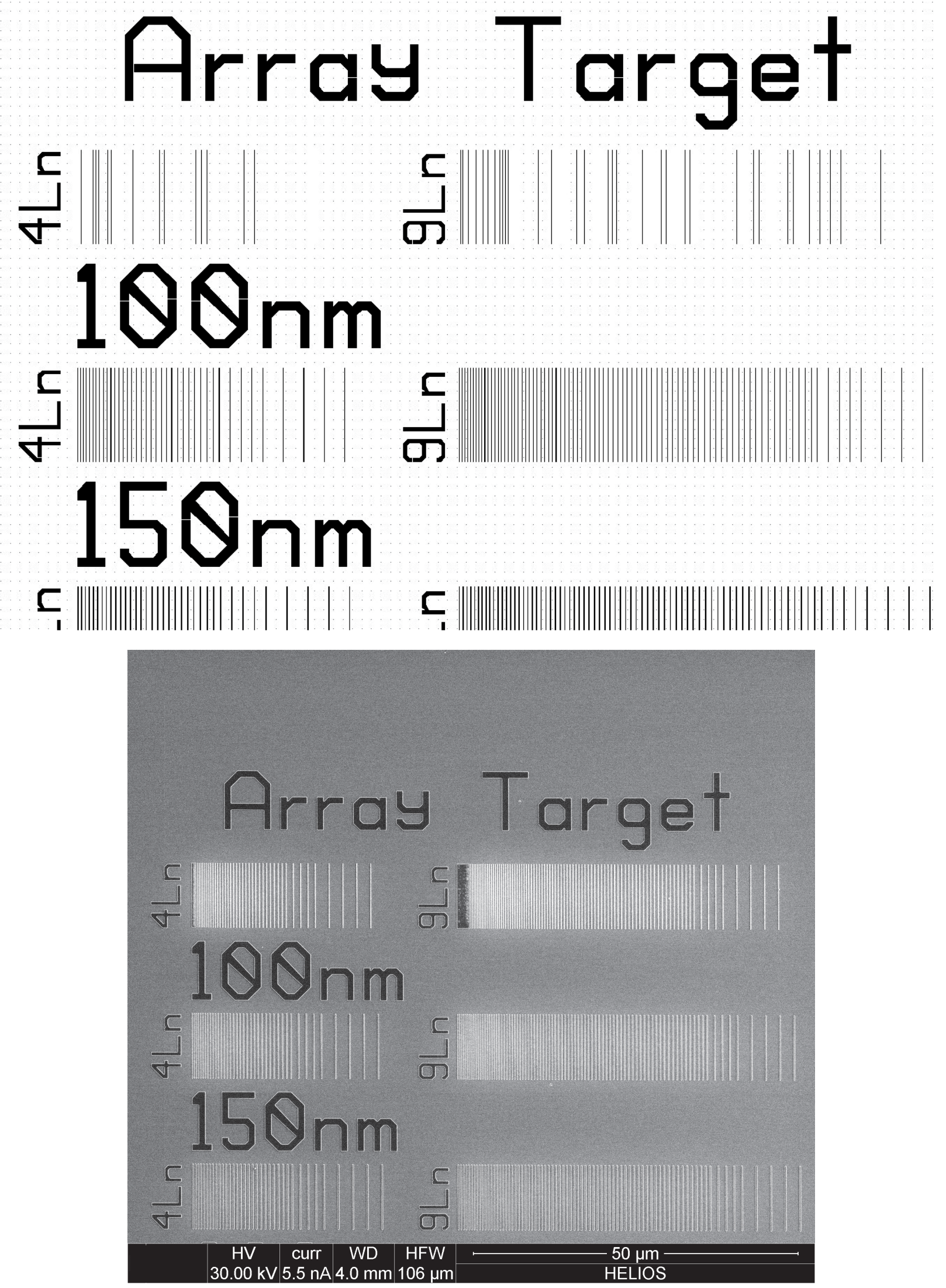

Plate 99 - Pattern 6-3 - Array Target. 
Plate 100 - Pattern 6-3 - Array Target. SEM micrograph of a segment of the Array Target pattern. 


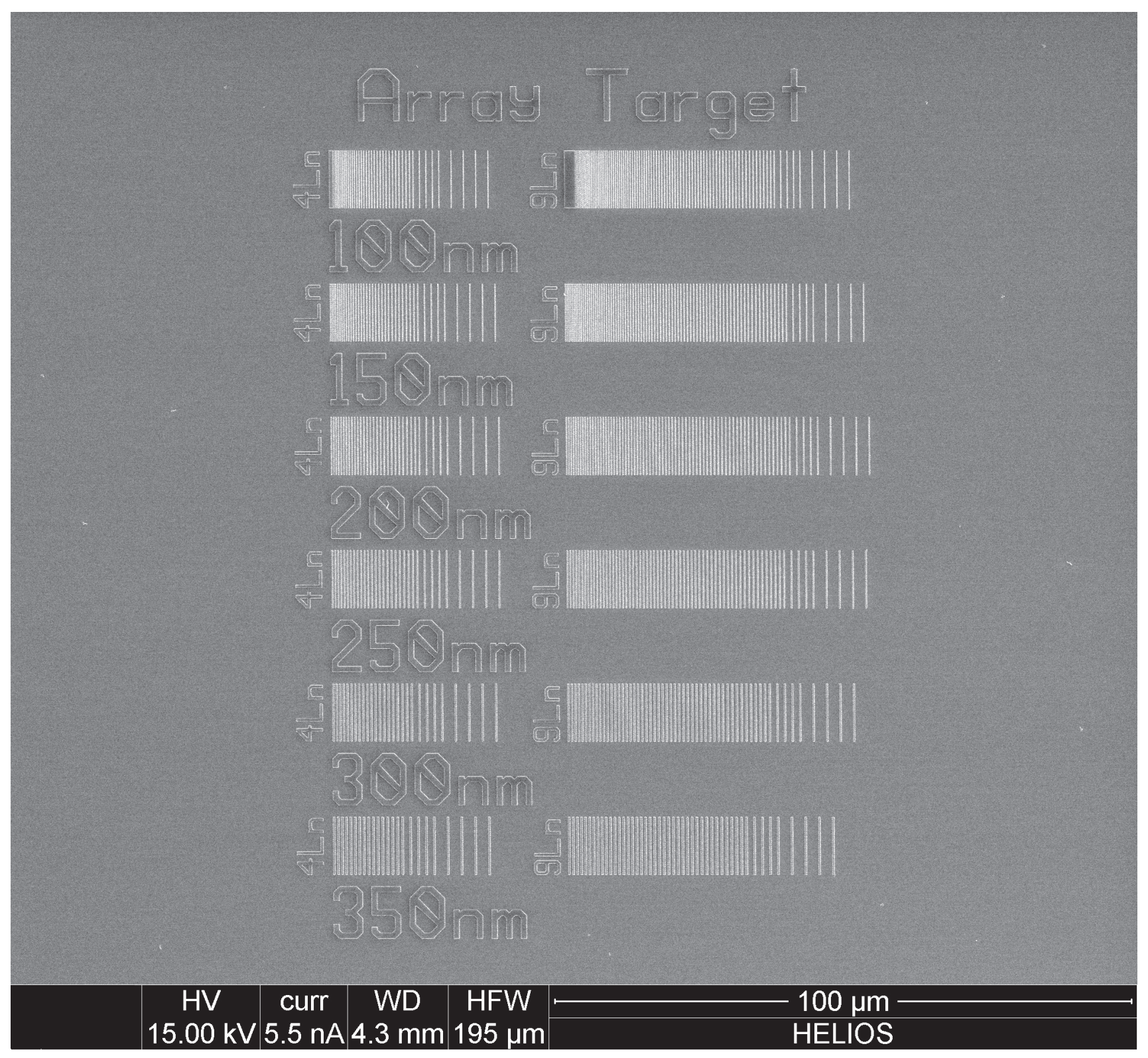


Plate 101 - Pattern 6-4 - Scatterometry 140 Module. (top) GDS II line drawing of the scatterometry 140 module module at a wide FOV. (bottom left and right) GDS II drawing of a reduced FOV of some of the fine detail of the patterns. 


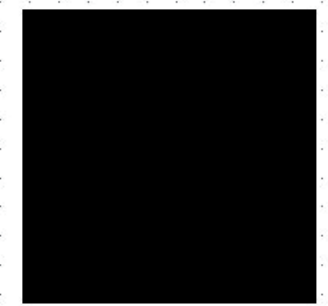

$100 \times 180$ um

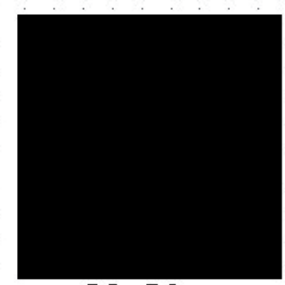

$98 \times 90 u m$

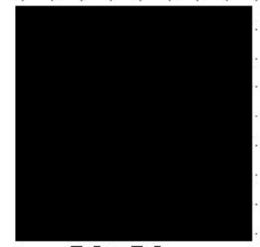

80x80um

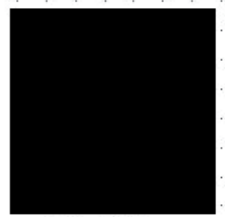

PQxPOum

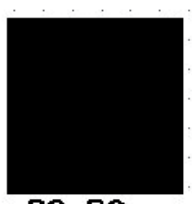

60x60um

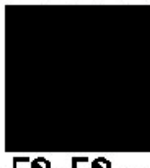

50x58um

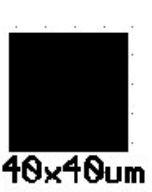

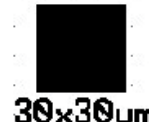

$20 \times 20$ um

10x10um
Soat

Target

$140 \mathrm{~nm}$

Module
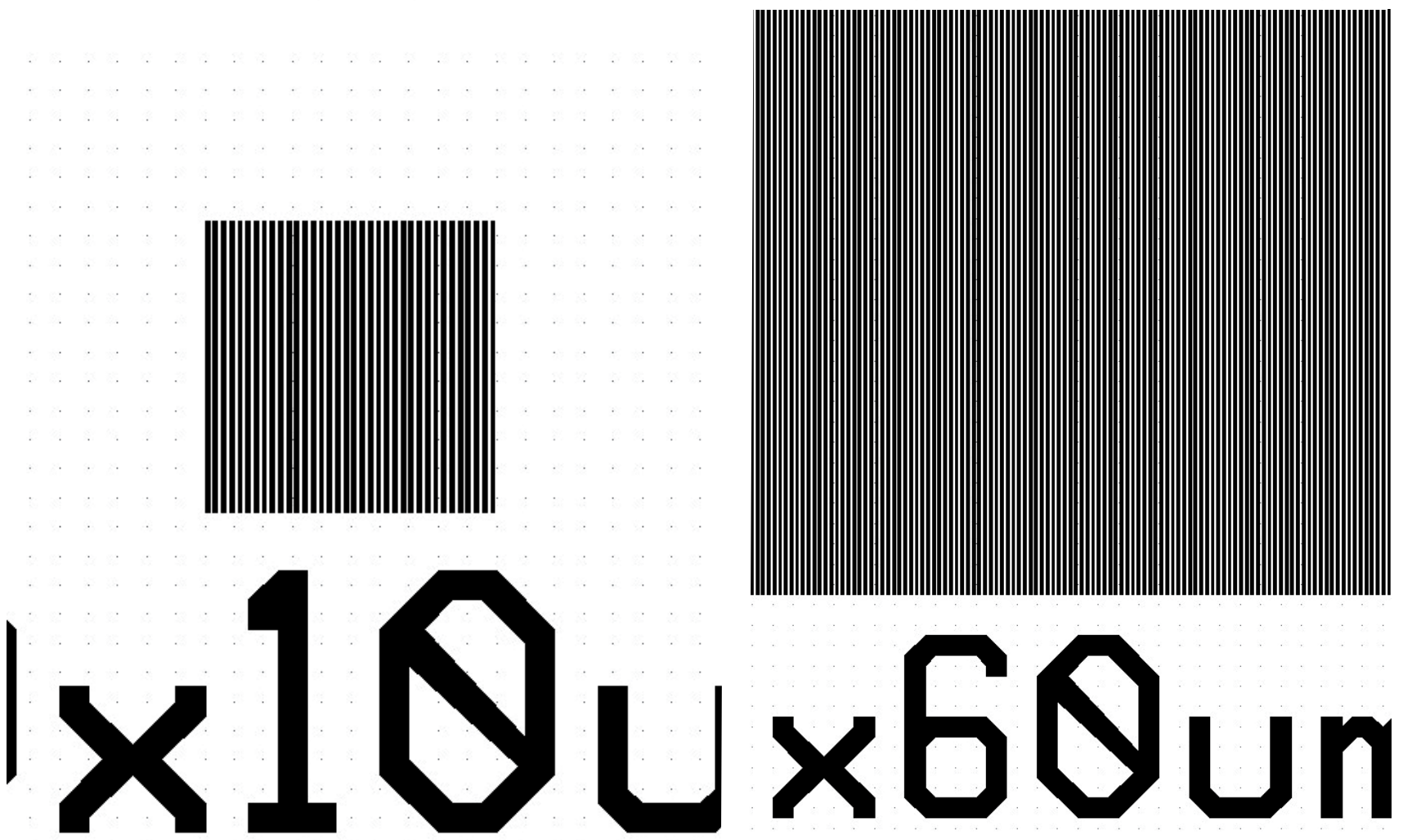

Plate 101 - Pattern 6-4 - Scatterometry 140 Module 
Plate 102 - Pattern 6-4 - Scatterometry 140 module. (top) SEM micrograph of the $100 \times 100$ array at low HFW (bottom) SEM micrograph of higher HFW showing the individual lines. 

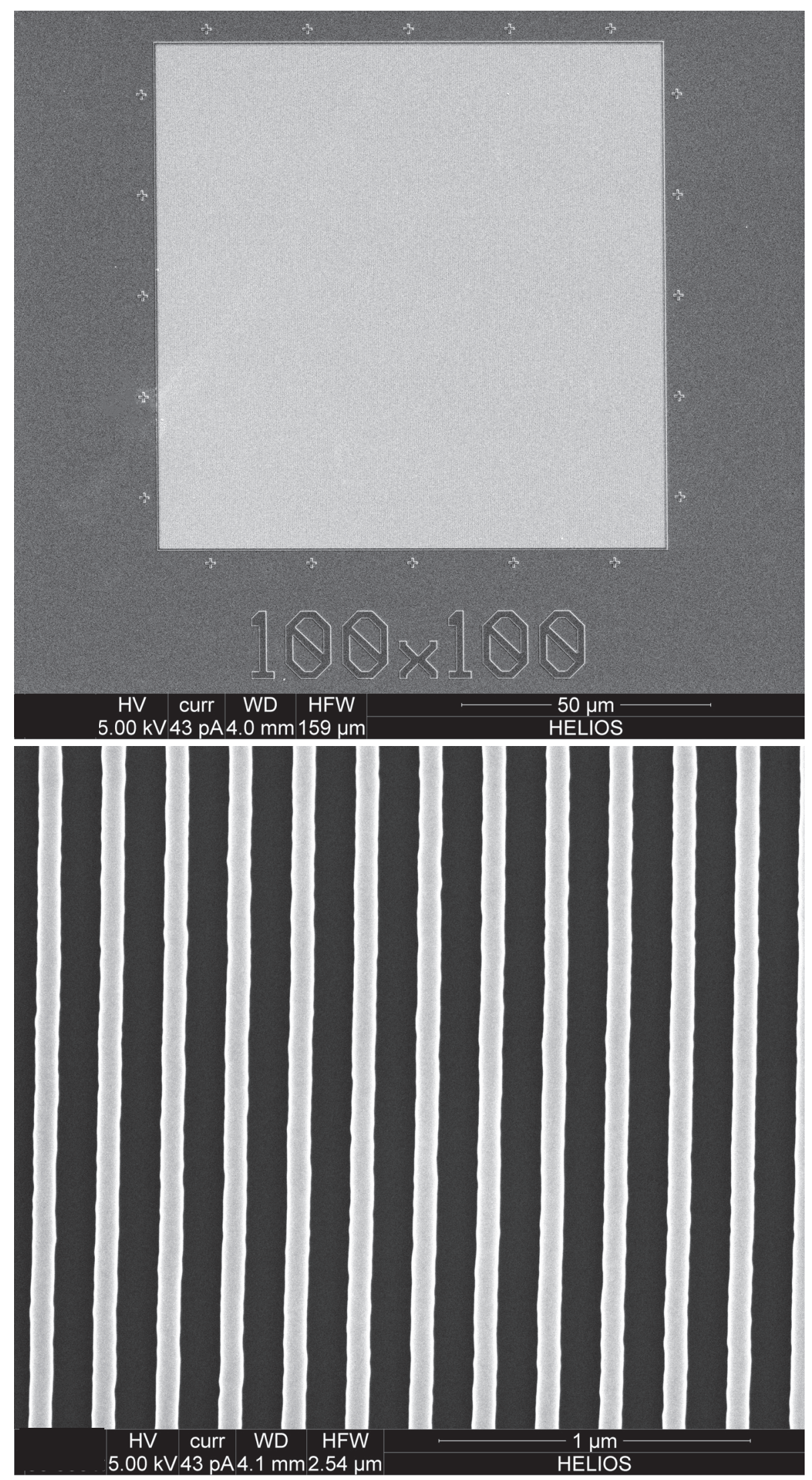

Plate 102 - Pattern 6-4 - Scatterometry 140 module 
Plate 103 - Pattern 6-5 - NIST (proposed) AFM Step Height Pattern. (top) GDS II line drawing of a portion of ta proposed NIST pattern for a step height standard module at a wide FOV. (bottom) SEM micrograph of the NIST pattern module showing the fine structure in the center.

\section{Explanation:}

NIST proposed step height prototype pattern. This pattern is designed to accommodate issues specific to AFMs. The large direction pattern surrounding the critical dimension target is to make locating the target easier for AFMs with limited navigation and pattern recognition capability. The large target and pitch size is for assessing large field of view scanning. (Designed by Ronald Dixson of NIST). 


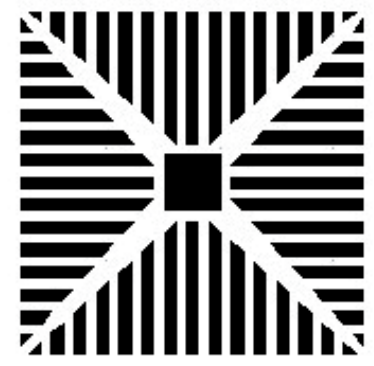

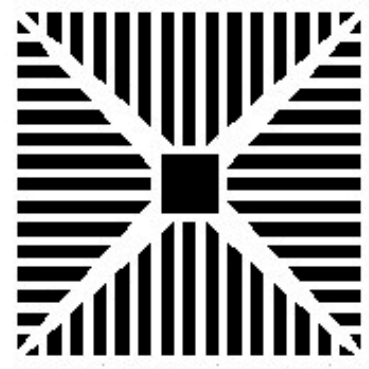

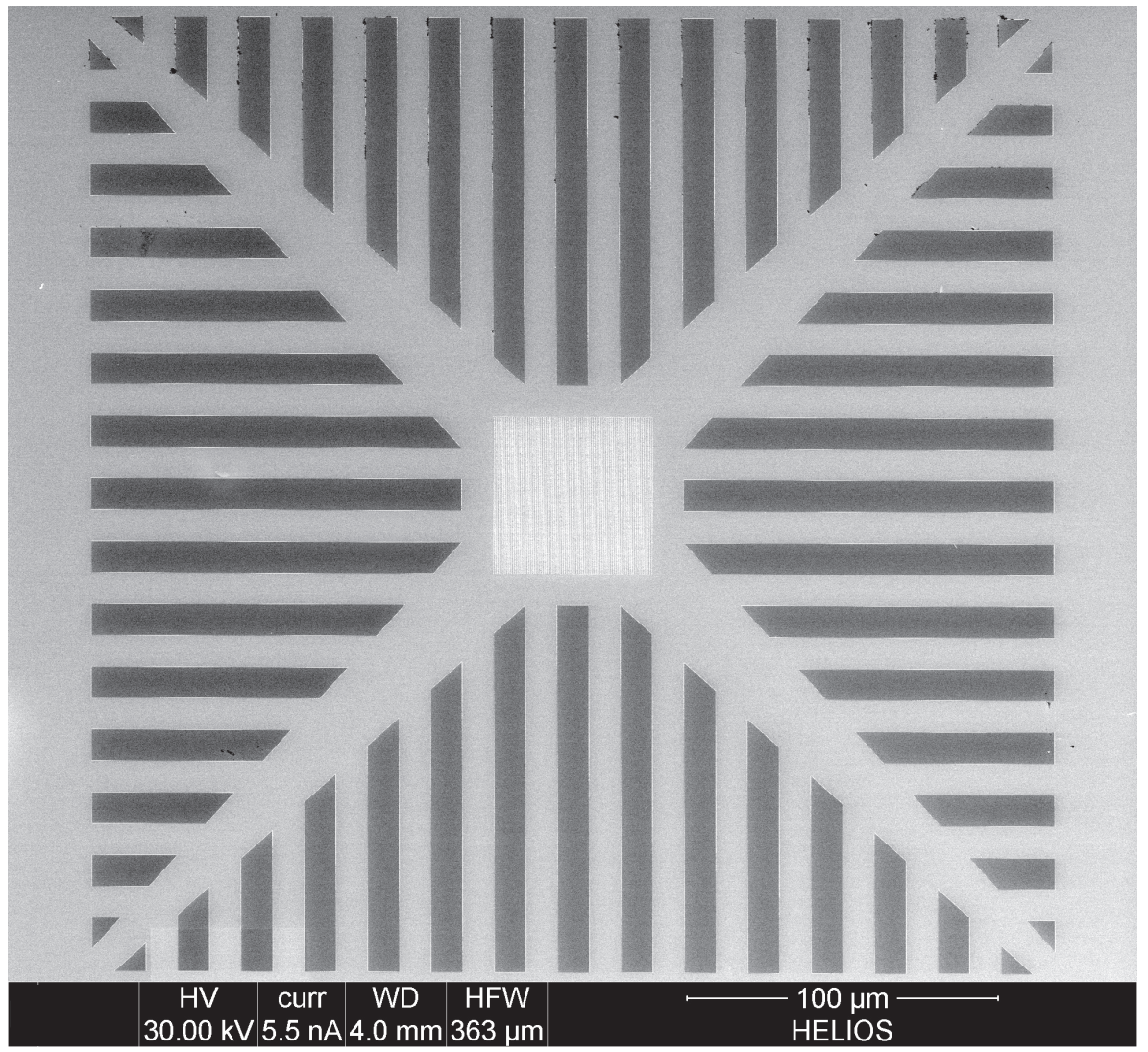

Plate 103 - Pattern 6-5 - NIST (proposed) AFM Step Height Pattern. 
Plate 104 - Pattern 6-5 - NIST (proposed) AFM Step Height Pattern. (top) GDS II line drawing of a portion of the NIST prototype pattern for step height standard module at a wide FOV. (bottom) SEM micrograph of the NIST pattern module showing the fine structure in the center. 

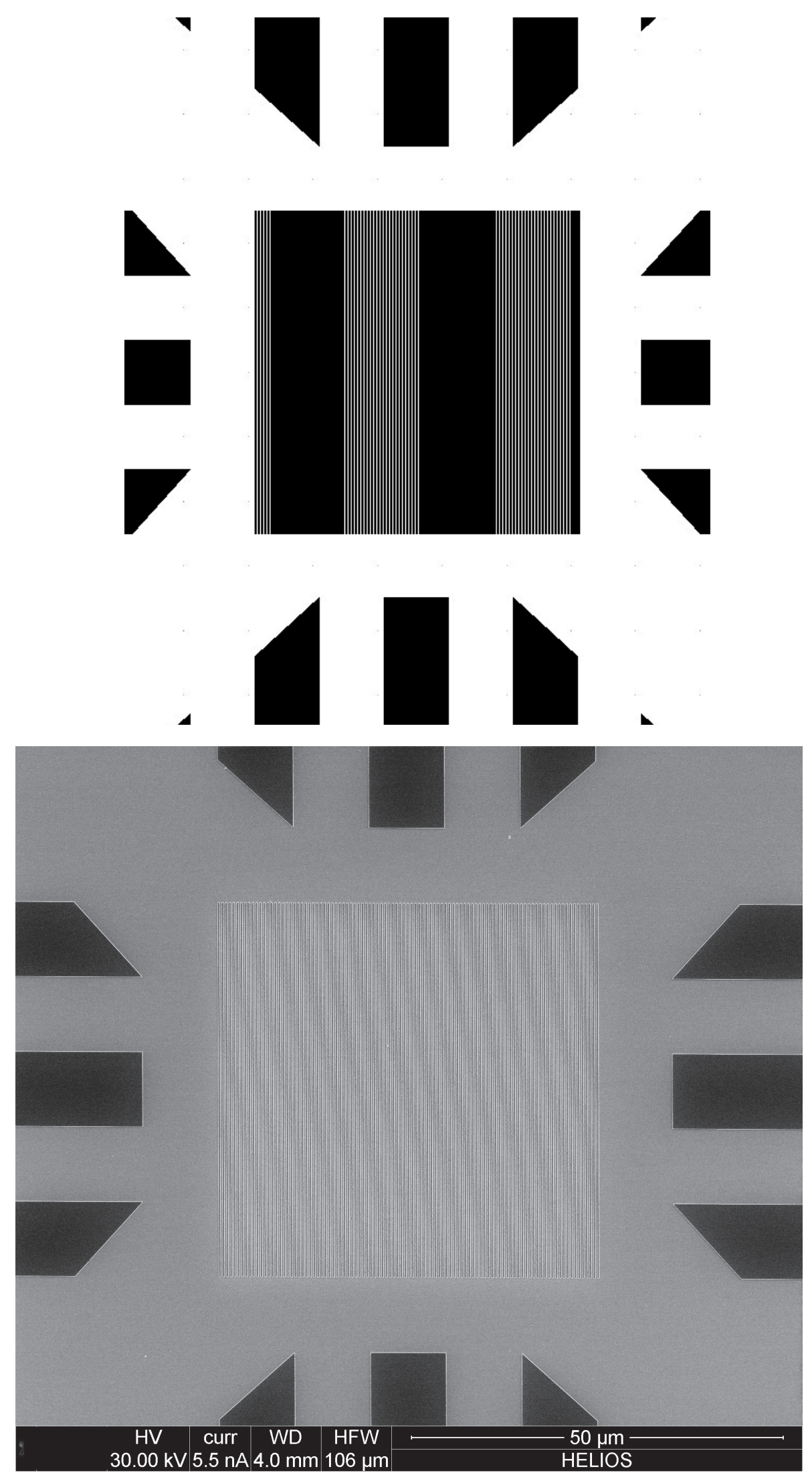
Plate 105. Pattern 6-5 NIST (proposed) AFM Step Height Pattern. SEM micrograph of the center of the NIST pattern module showing the fine structure. 


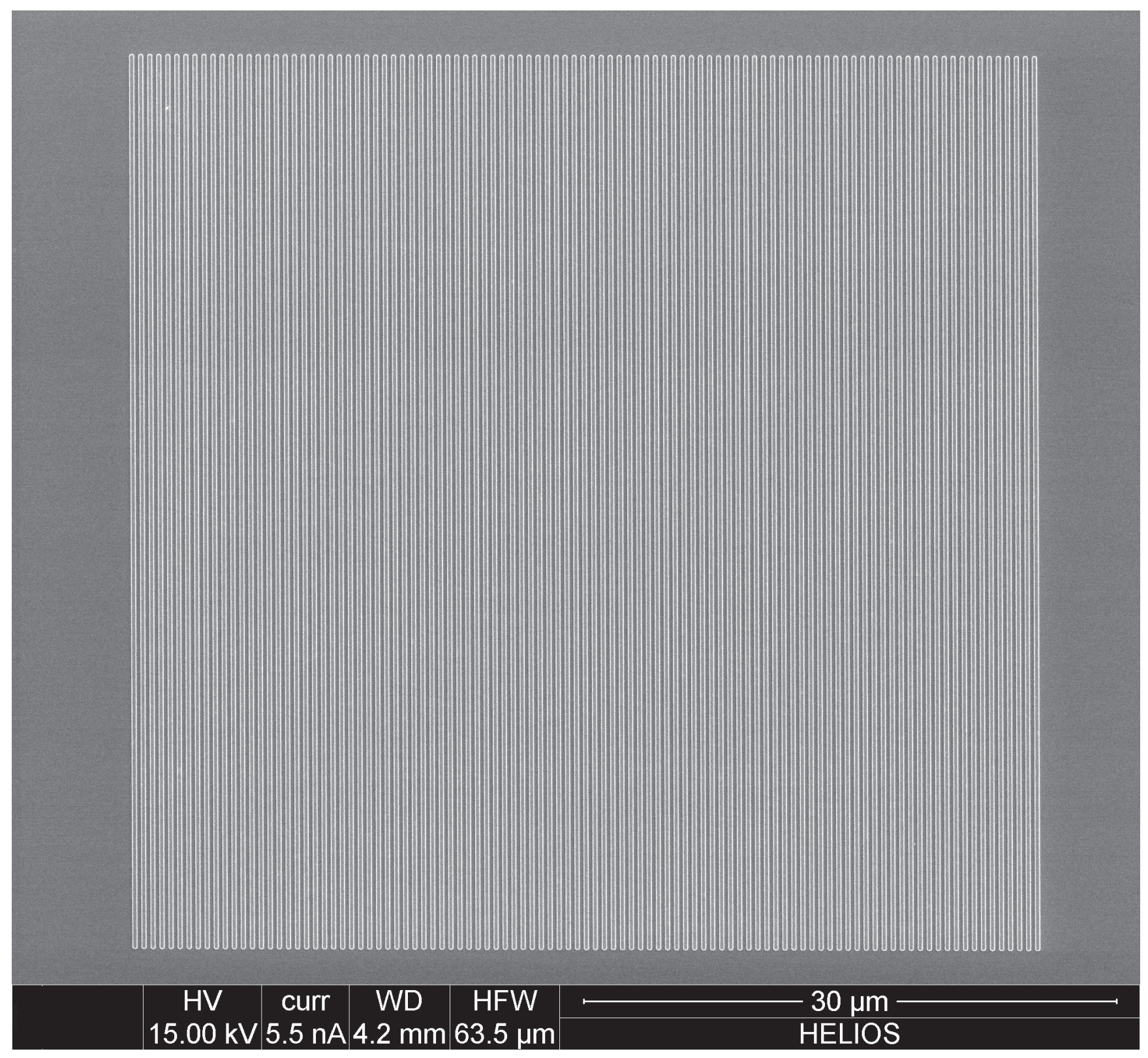


Plate 106 - Pattern 6-6 - High Resolution Overlay. (top) GDS II line drawing of the high resolution overlay targets module Pattern 6-8 and 6-9 at a wide FOV. (bottom) SEM micrograph of a similar area.

\section{Explanation:}

High Resolution Overlay Targets. The High Resolution Module is made of several 20 x 20 micrometer boxes of line/trench modules. CDs are $100 \mathrm{~nm}, 150 \mathrm{~nm}, 200 \mathrm{~nm}, 250 \mathrm{~nm}, 300 \mathrm{~nm}, 400$ $\mathrm{nm}, 500 \mathrm{~nm}$. The space widths are $500 \mathrm{~nm}, 1000 \mathrm{~nm}, 2000 \mathrm{~nm}, 3000 \mathrm{~nm}, 4000 \mathrm{~nm}, 5000 \mathrm{~nm}$. There are three cells for each CD/space: the first cell has a $30 \%$ offset in the positive $\mathrm{X}$ axis, the second cell has a $30 \%$ offset in the positive Y axis, and the third cell has no offset but is 180 degrees out of phase. Because this pattern was originally intended for an overlay reticle set, only a single level target is displayed. 

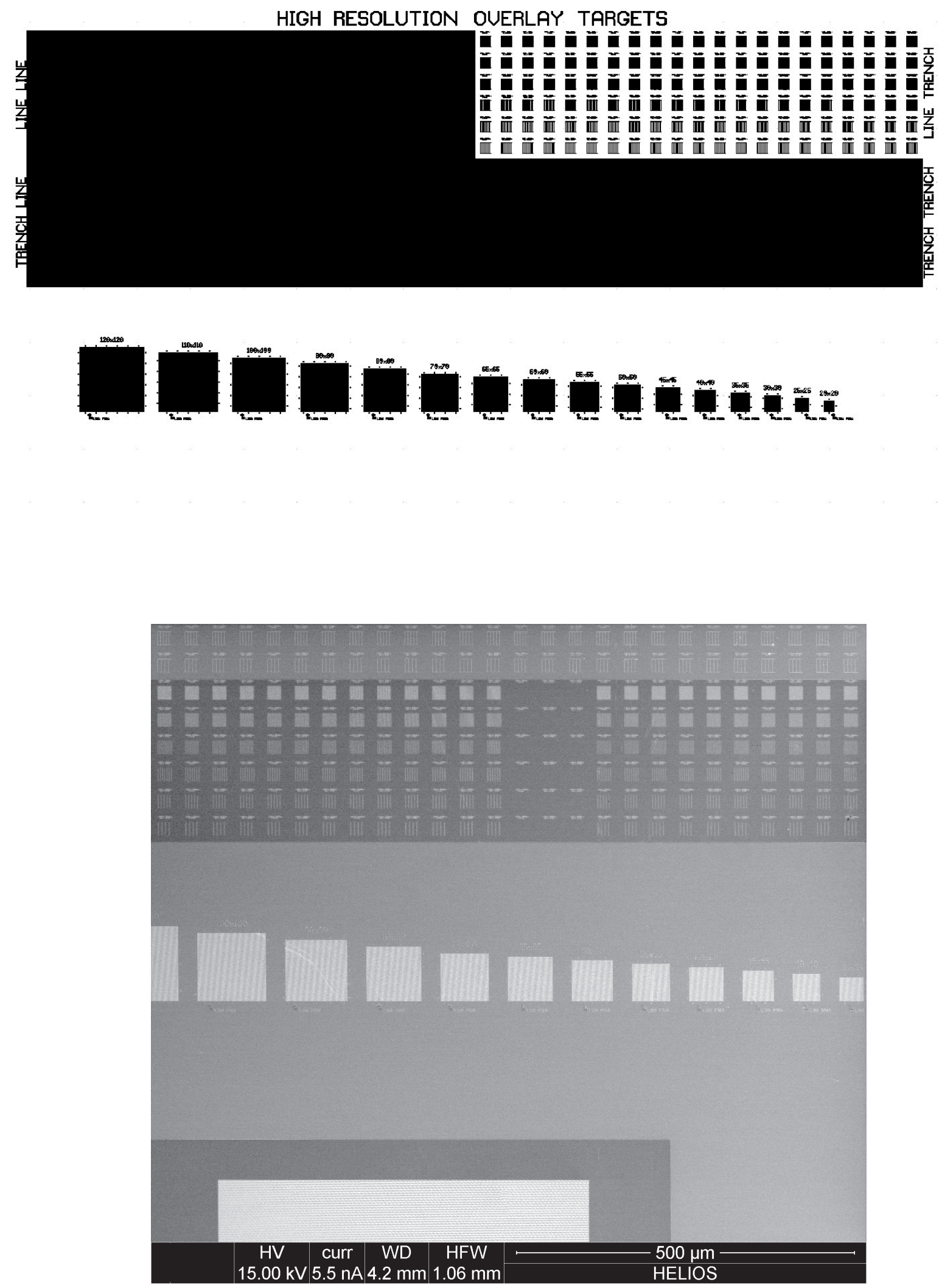

Plate 106 - Pattern 6-6 - High Resolution Overlay 
Plate 107 - Pattern 6-6 - High Resolution Overlay. (top) GDS II line drawing of the high resolution overlay targets module Pattern 6-6 at a wide FOV. (bottom) SEM micrograph of a similar area. 


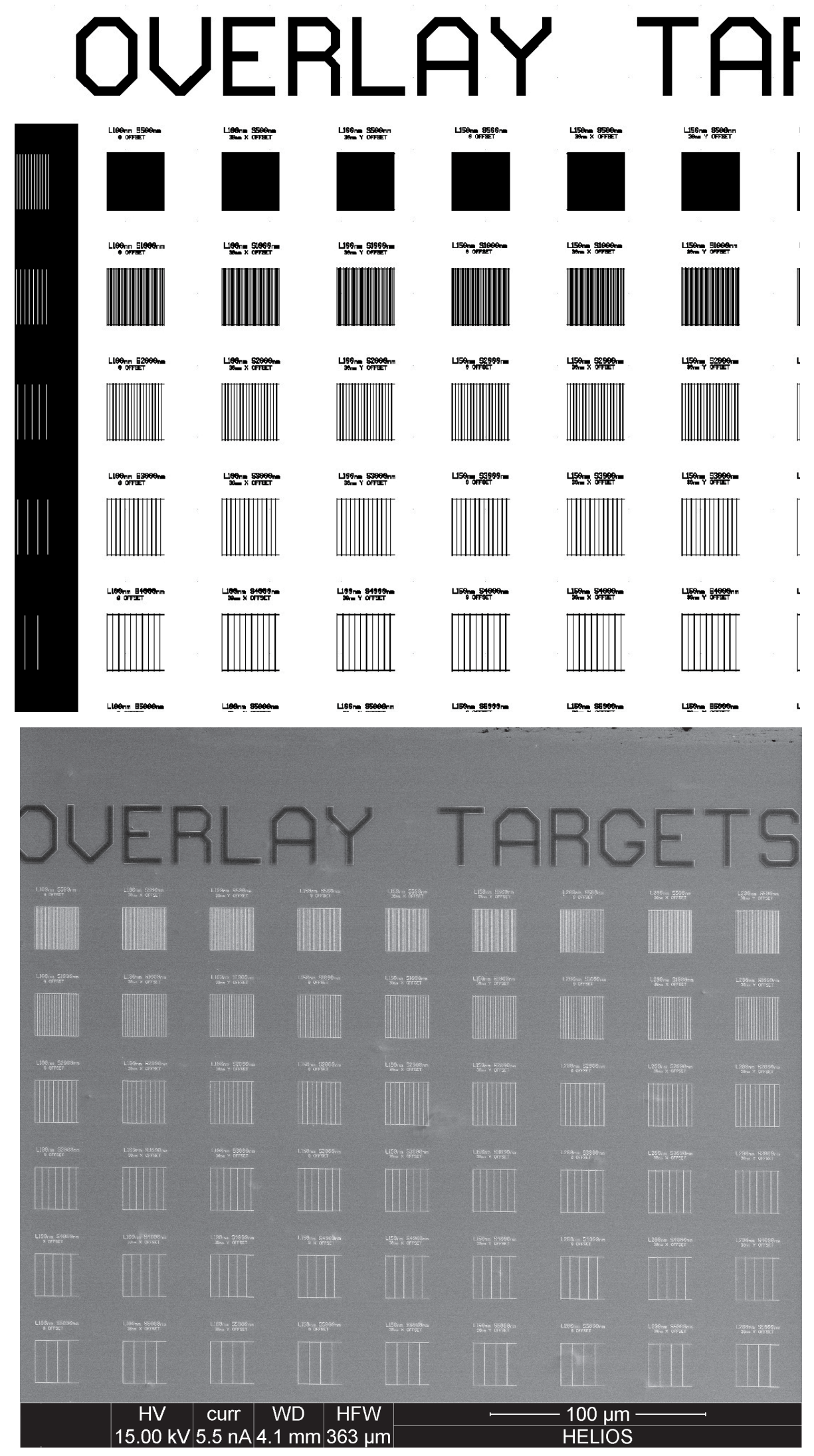

Plate 107 - Pattern 6-6 - High Resolution Overlay 
Plate 108 - Pattern 6-6 - High Resolution Overlay. (top) GDS II line drawing of the high resolution overlay targets module Pattern 6-6 at a wide FOV. (bottom) SEM micrograph of a similar area. 


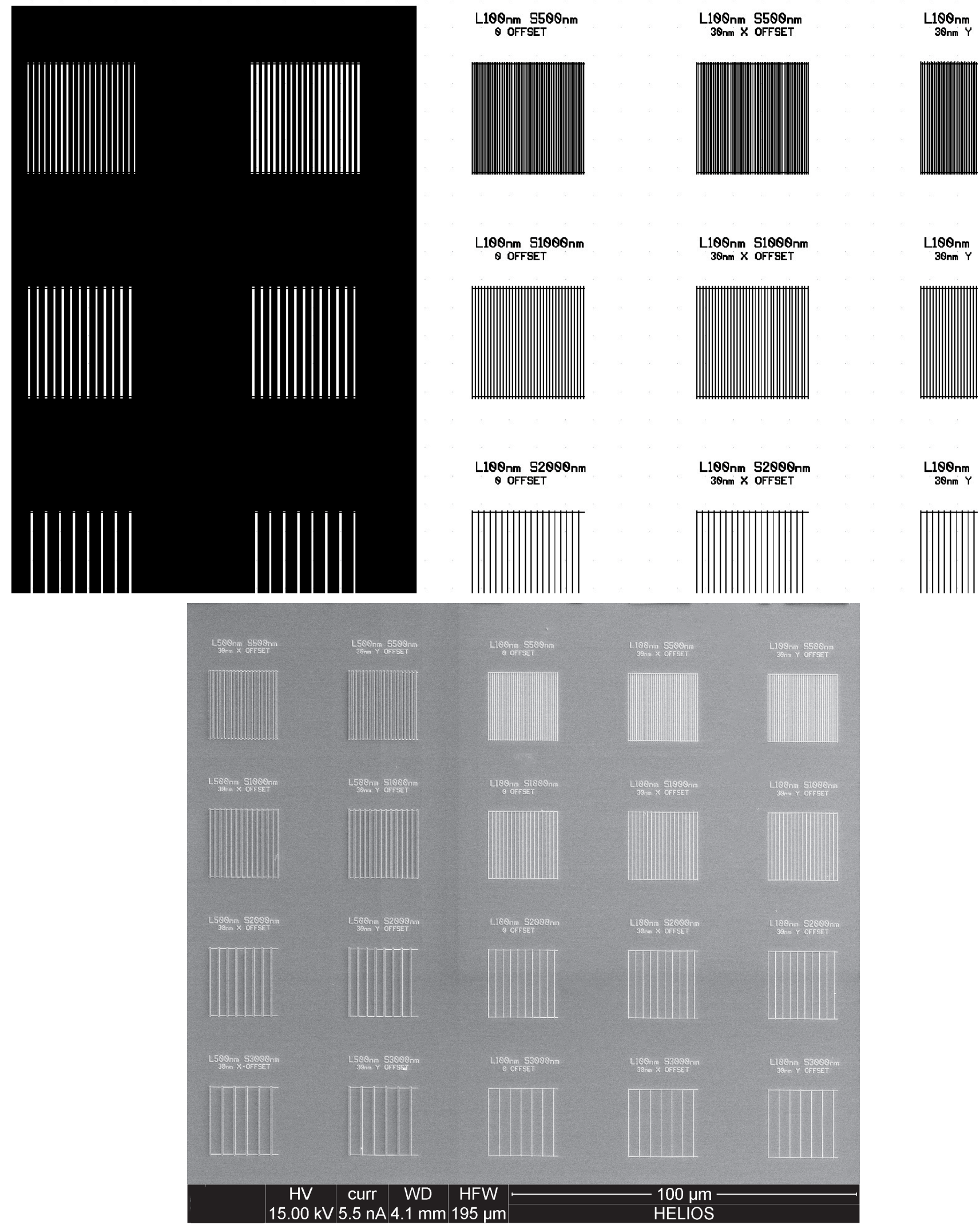

Plate 108 - Pattern 6-6 - High resolution overlay 
Plate 109 - Pattern 6-6 - High Resolution Overlay. (top and bottom) SEM micrographs of the overlay patterns at higher HFW. 


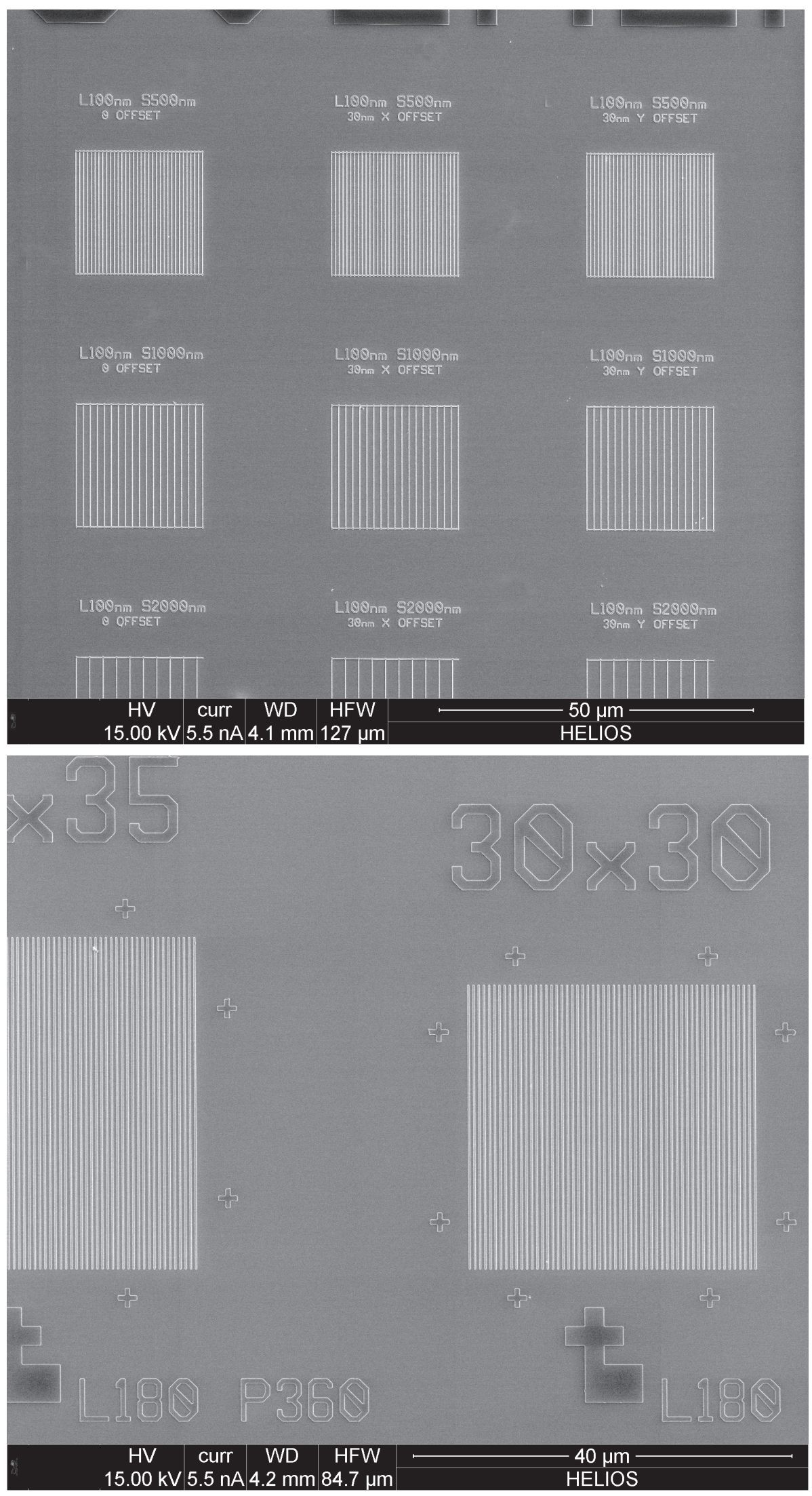

Plate 109 - Pattern 6-6 - High Resolution Overlay 
Plate 110 - Pattern 6-6 - High Resolution Overlay. (top) GDS II line drawing of the high resolution overlay targets module Pattern 6-6 at a wide FOV. (bottom) SEM micrograph of a similar area. 


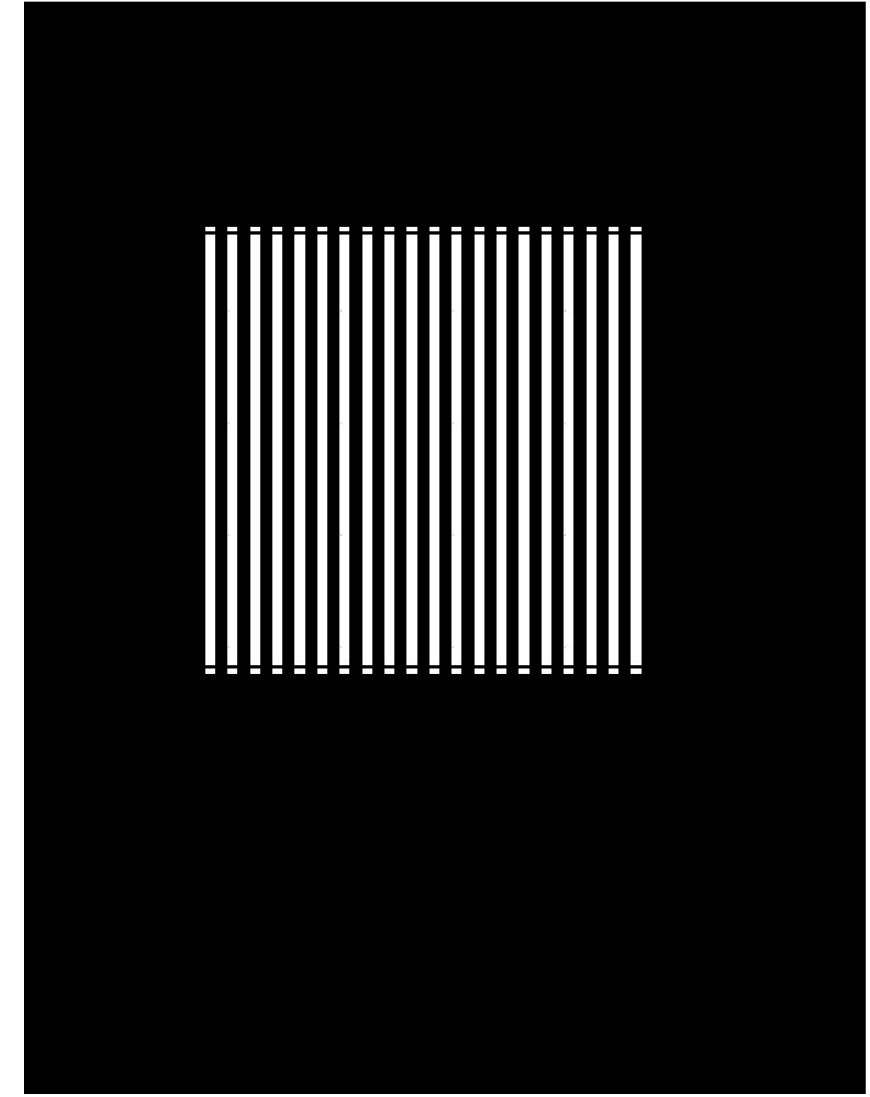

\section{L100nm S500nm $\theta$ OFFSET}

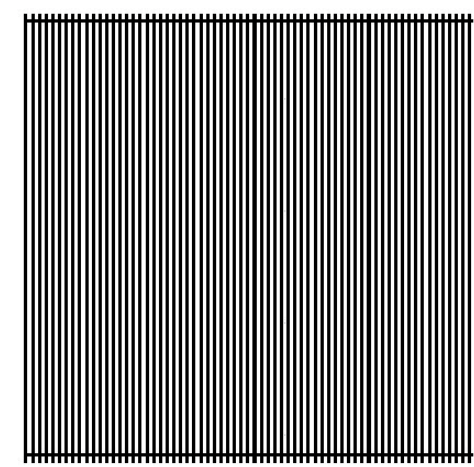

\section{$\mathrm{L} 100 \mathrm{~nm} 51000 \mathrm{~nm}$} $\theta$ OFFSET

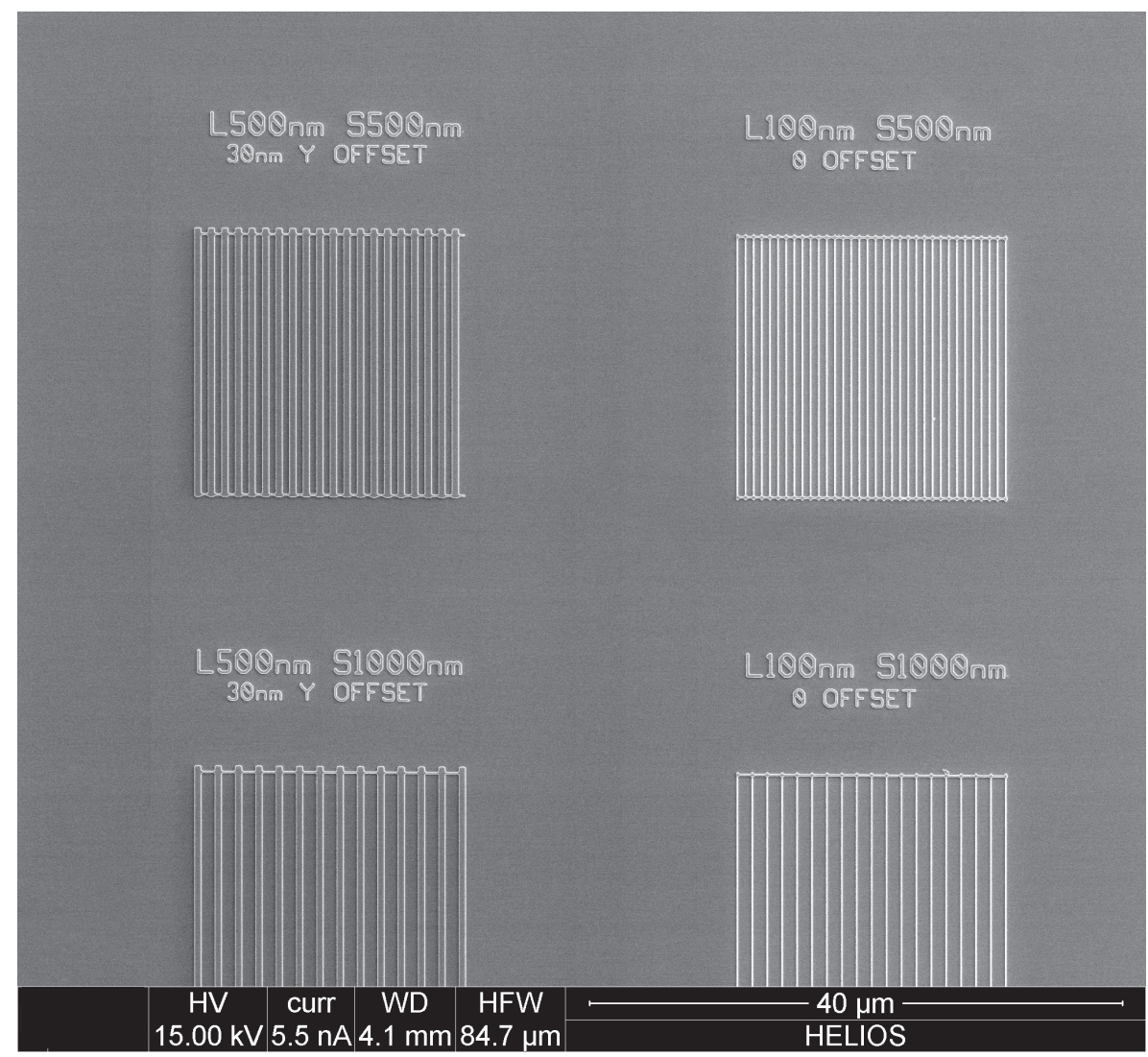

Plate 110 - Pattern 6-6 - High Resolution Overlay. 
Plate 111 - Pattern 6-7 - Scatterometry Field Size. (top) Reduced field GDS II line drawing of several of the scatterometry targets found on Pattern 6-7 at a reduced FOV. (bottom) SEM micrograph of a similar area. 


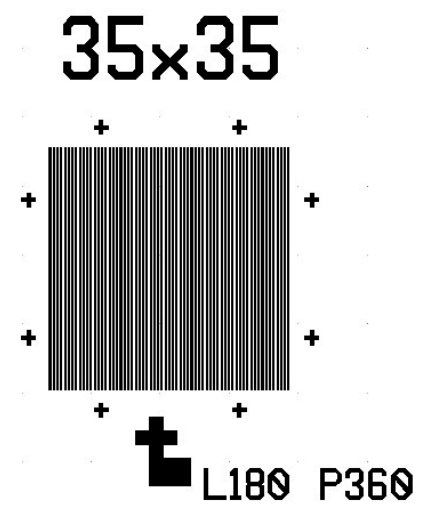

\section{$30 \times 30$}

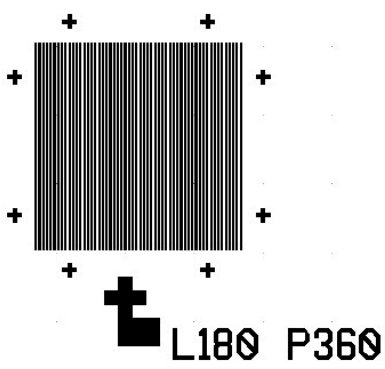

$25 \times 25$

$+$

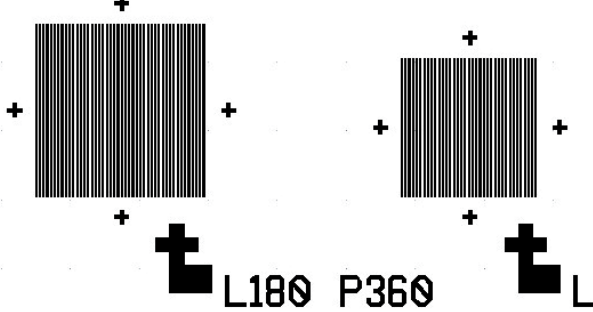

$2 \theta \times 2 \theta$

L180 P360

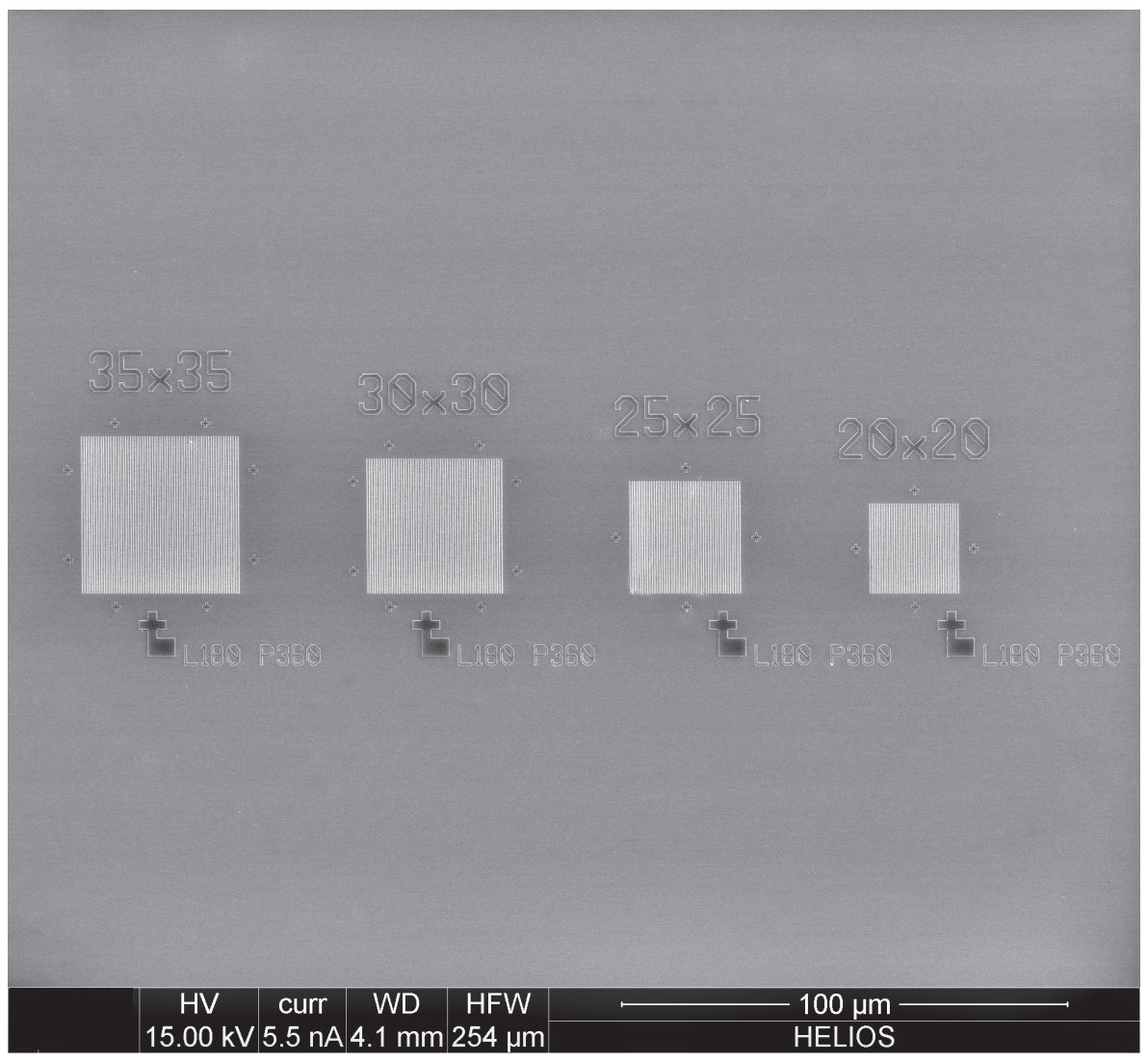

Plate 111 - Pattern 6-7 - Scatterometry Field Size 
Plate 112 - Pattern 6-7 - Scatterometry Field Size. (top) Reduced field GDS II line drawing of several of the scatterometry targets found on Pattern 6-7 at a reduced FOV. (bottom) SEM micrograph of a similar area. 


\section{$35 \times 35$}
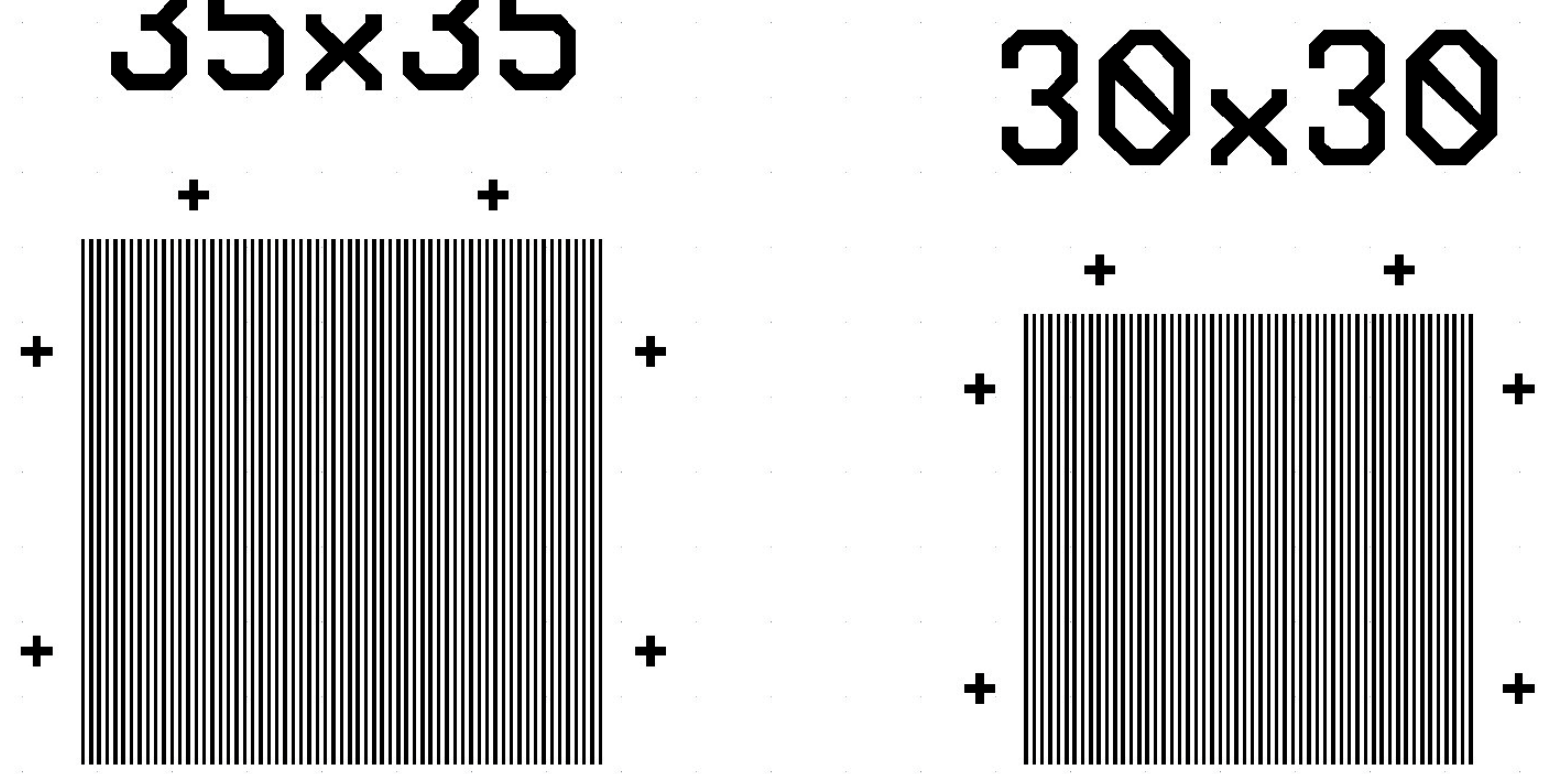

9
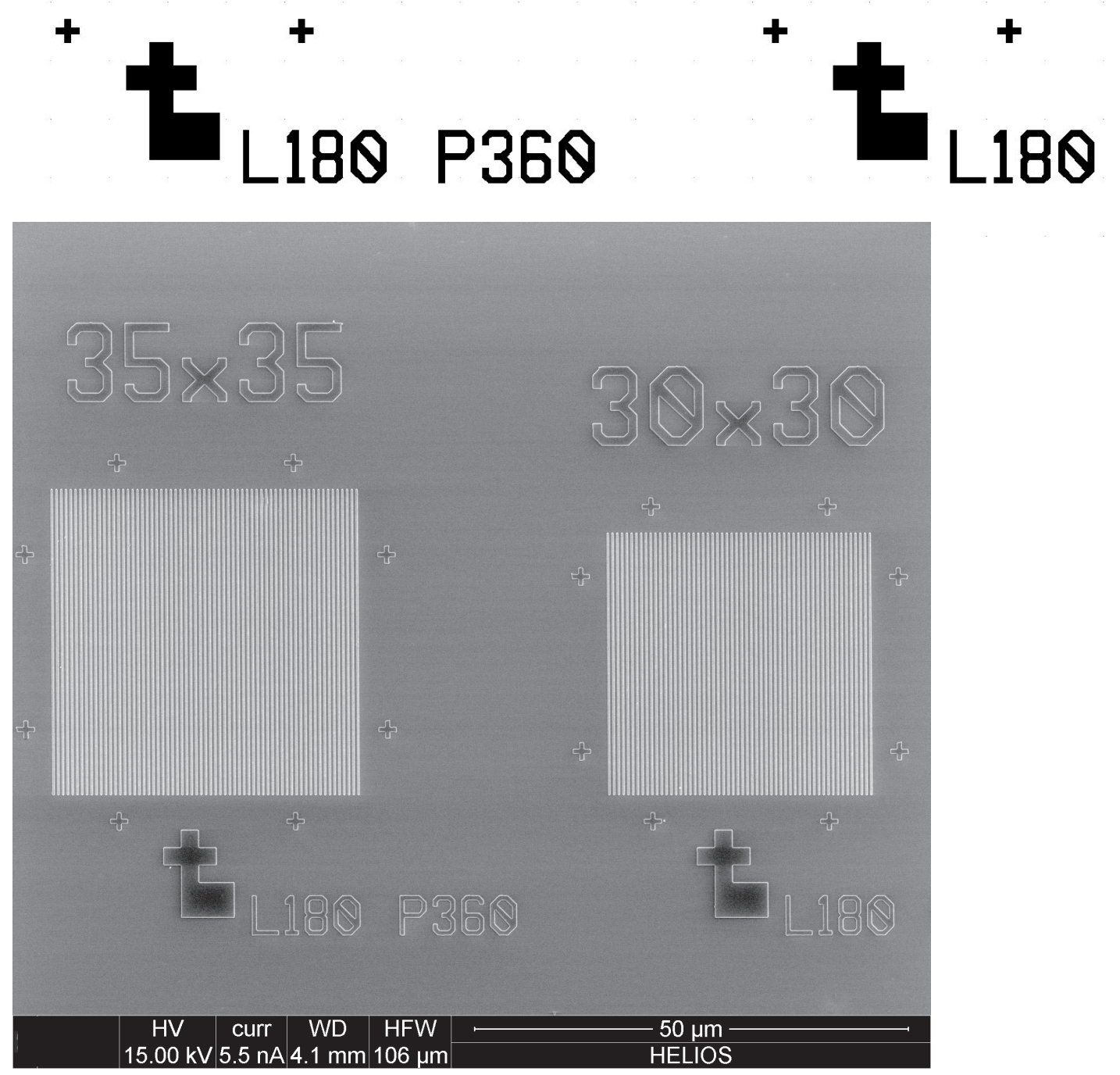

Plate 112 - Pattern 6-7 - Scatterometry Field Size. 
Page intentionally left blank 
SECTION 7 
Plate 113. Section 7-0 Overall. (top) GDS II line drawing of the entire RM 2089 pattern highlighting Section 7 with the 6 additional major sub-patterns numerically identified. (bottom) GDS II detail drawing of section 7 showing the 6 additional major sub-patterns included in this section. Pattern 7-1 is also seen in the center of this module. 

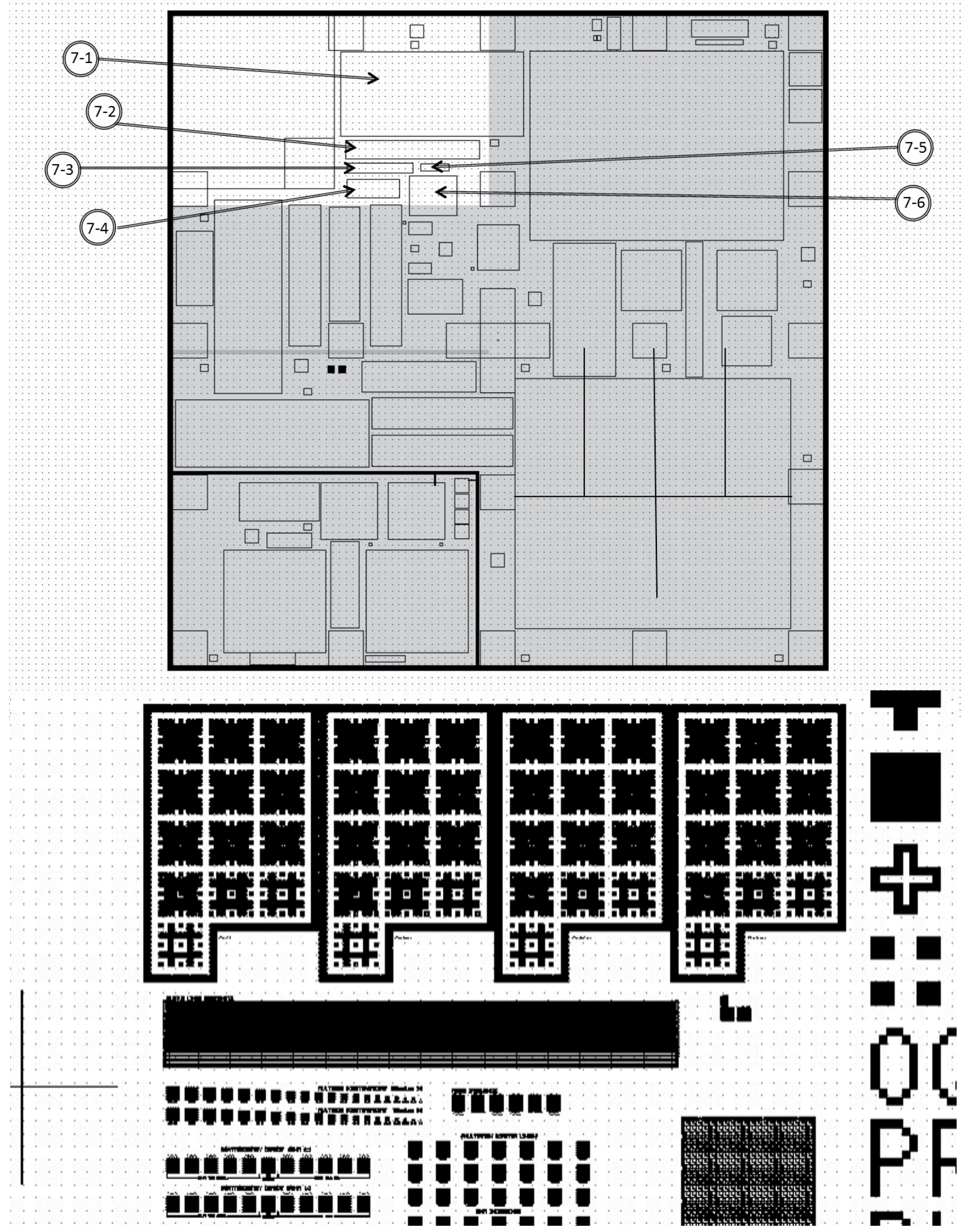

Plate 113. Section 7-0 Overall. 
Plate 114 - Patterns 7-1 and 7-2. Wide FOV of GDS II line drawing of Patterns 7-1 (Fill module) and 7-2 (Cleave lines) at wide FOV. 

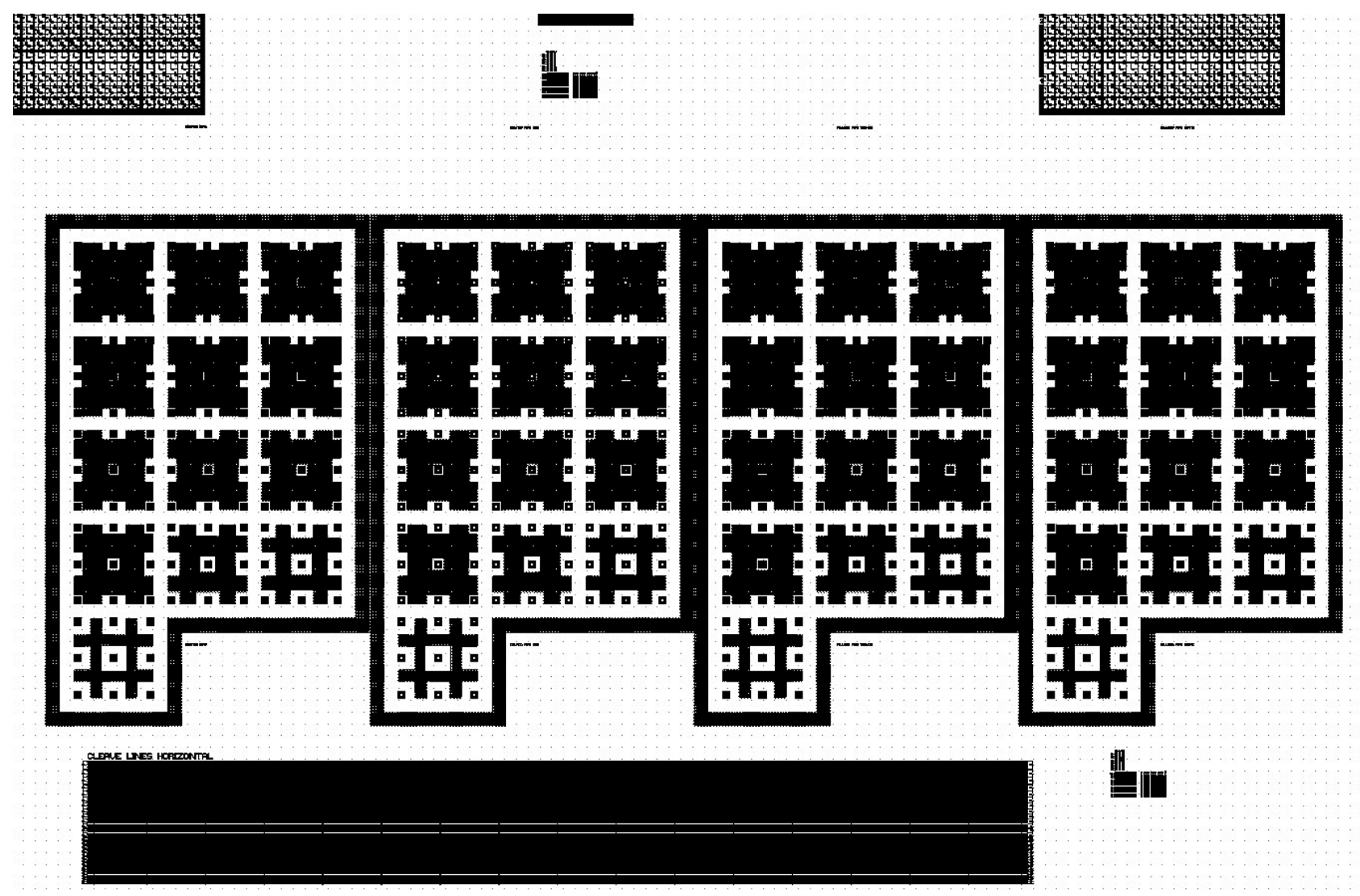

Plate 114 - Patterns 7-1 and 7-2. 
Plate 115 - Patterns 7-1 - Fill Module. (top) Wide FOV of GDS II line drawing of patterns 7-1 . fill module. (below). SEM micrograph of a similar area.

\section{Explanation:}

Fill Module. Each module contains 13 sub modules as shown in Pattern 7-1. In each sub module there is dummy fill surrounding 9 targets. There are four different types of targets but in each module only one type is used. The first module contains line in dense line targets, the second module contains box in box targets, the third module contains $0.5 \mu \mathrm{m}$ trench in trench targets, and the fourth module contains $1 \mu \mathrm{m}$ frame in frame targets. The targets remain the same in each module while the dummy fill expands away from each overlay target. The fill remains a constant. $100 \mu \mathrm{m}$ away for the outer centered targets, the fill then varies from sub module to sub module. Sub modules go from left to right, top to bottom. One thing to note is that because this cell was originally intended for an overlay reticle set, you will only see a single level target. 

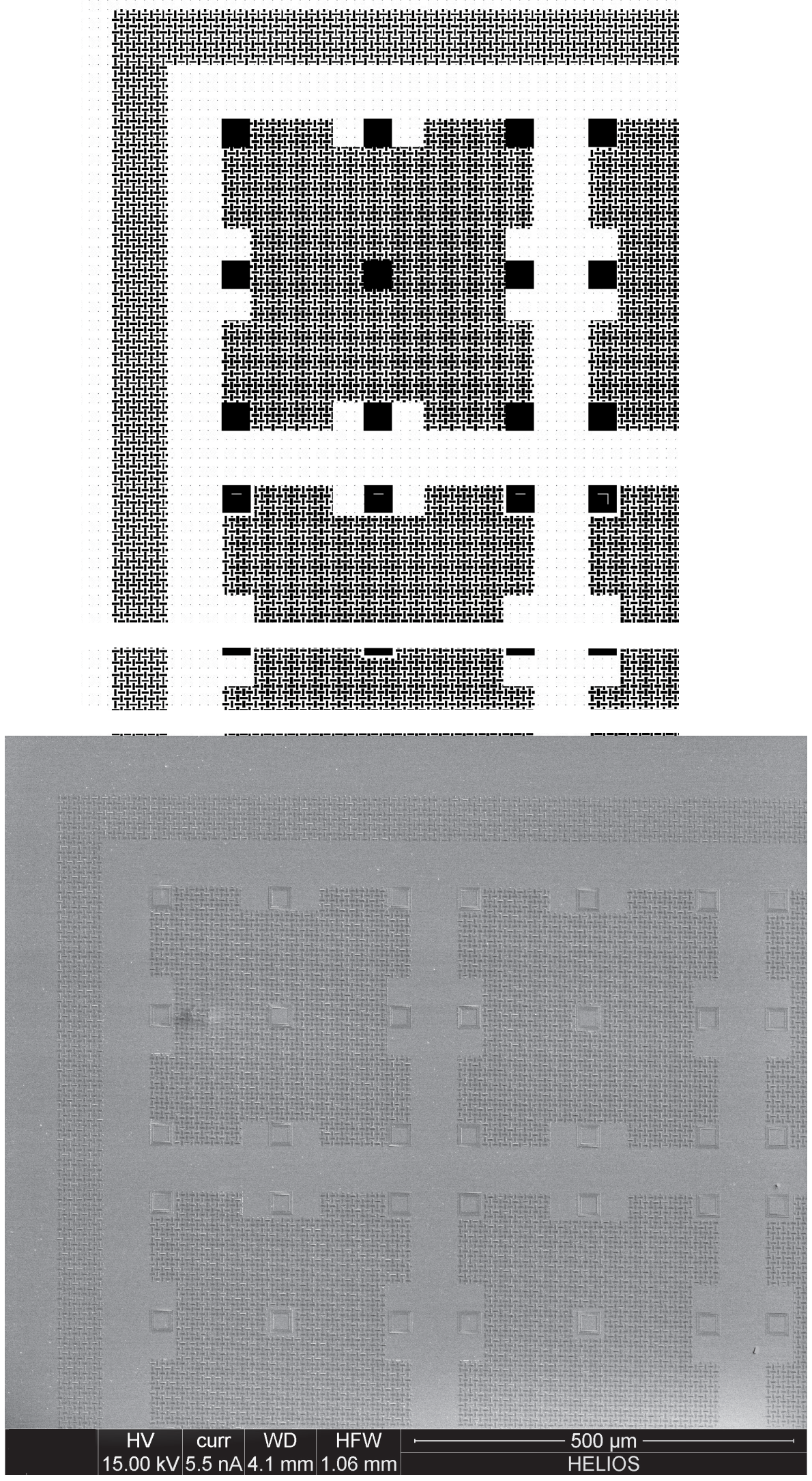

Plate 115 - Patterns 7-1 - Fill Module 
Plate 116 - Patterns 7-1 - Fill Module. (top) Low HFW SEM micrograph of the fill module. (bottom) Higher HFW micrograph of a one of the cells. 

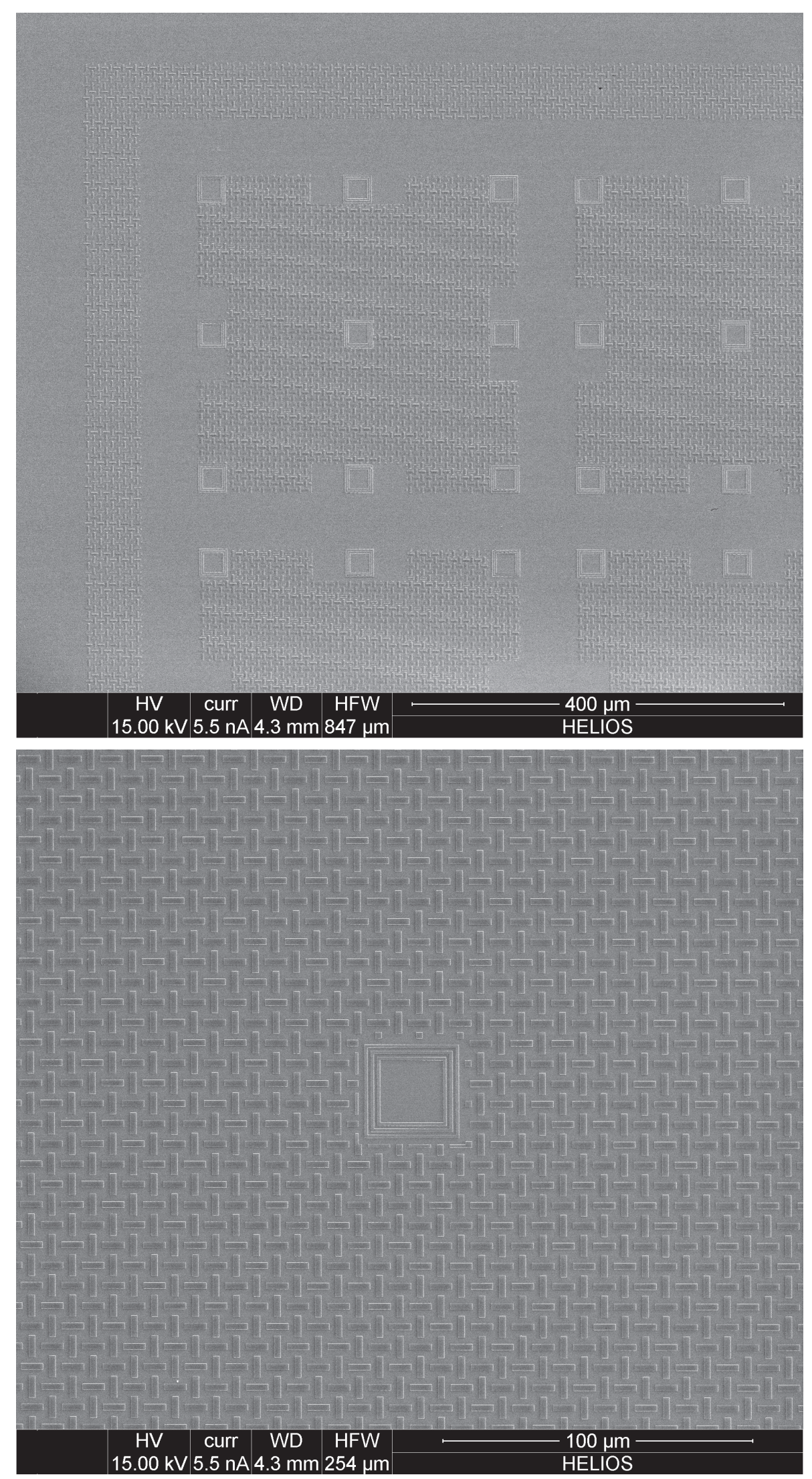

Plate 116 - Patterns 7-1 - Fill Module. 
Plate 117 - Patterns 7-1 - Fill Module. (top) Low HFW SEM micrograph of the fill module. (bottom) Higher HFW micrograph of a one of the cells. 


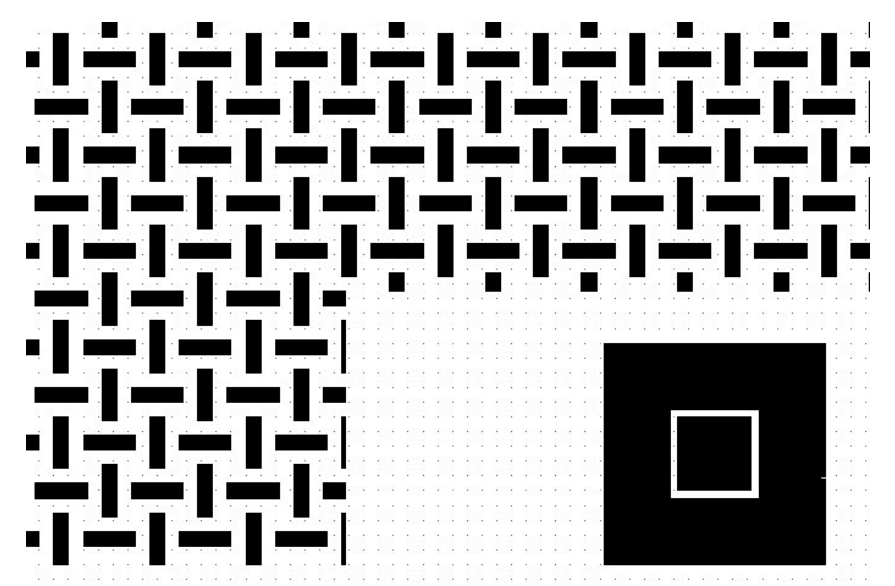

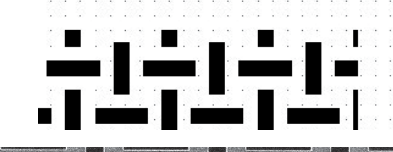
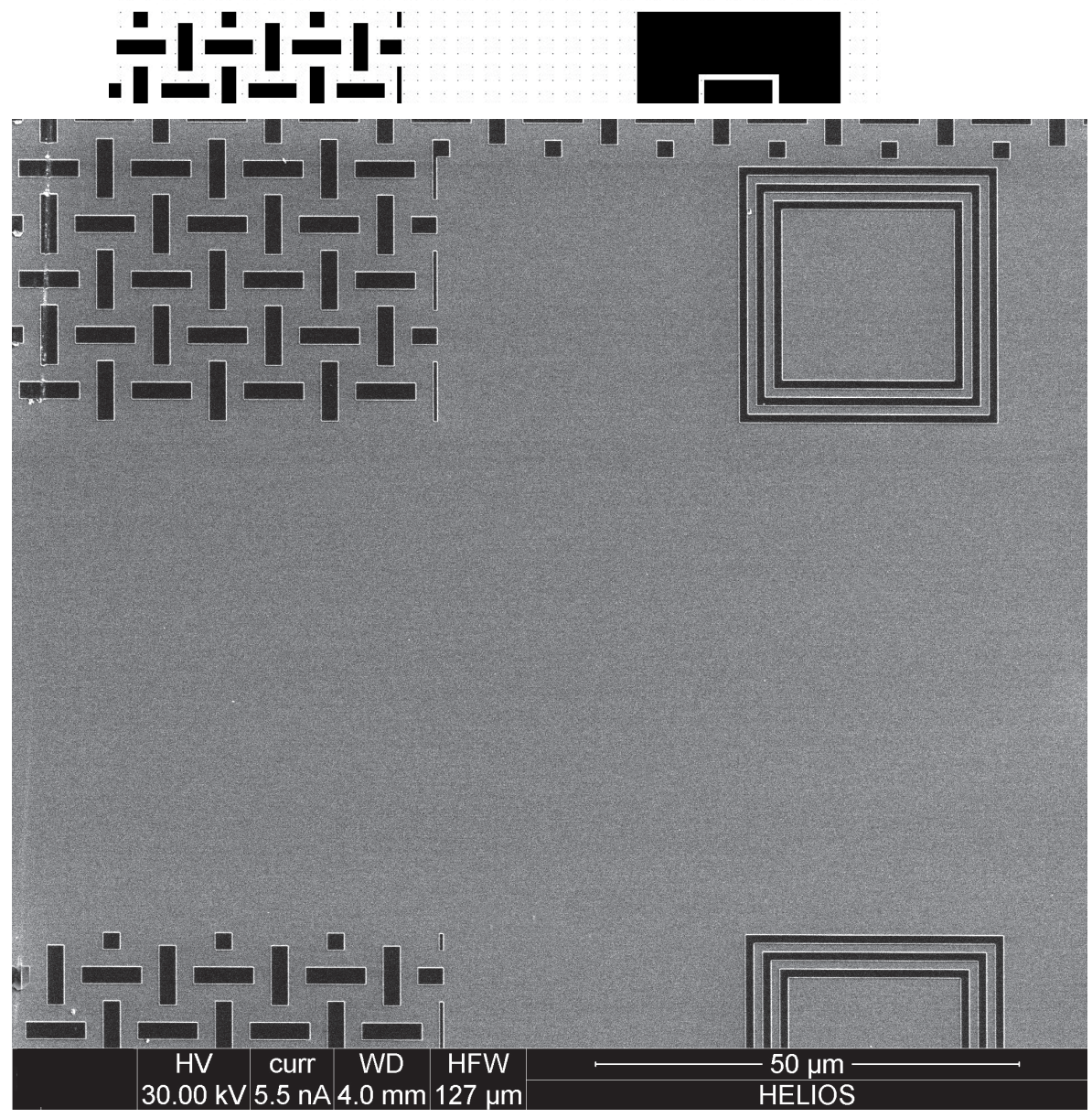

Plate 117 - Patterns 7-1 - Fill Module. 
Plate 118 - Pattern 7-2 - Cleave Lines. (top) Reduced FOV GDS II line drawings of several of the horizontal cleave line targets found on Pattern 7-2. (bottom) SEM micrograph of a similar area. 


\section{CLEAUE LINES HORIZONTAL}
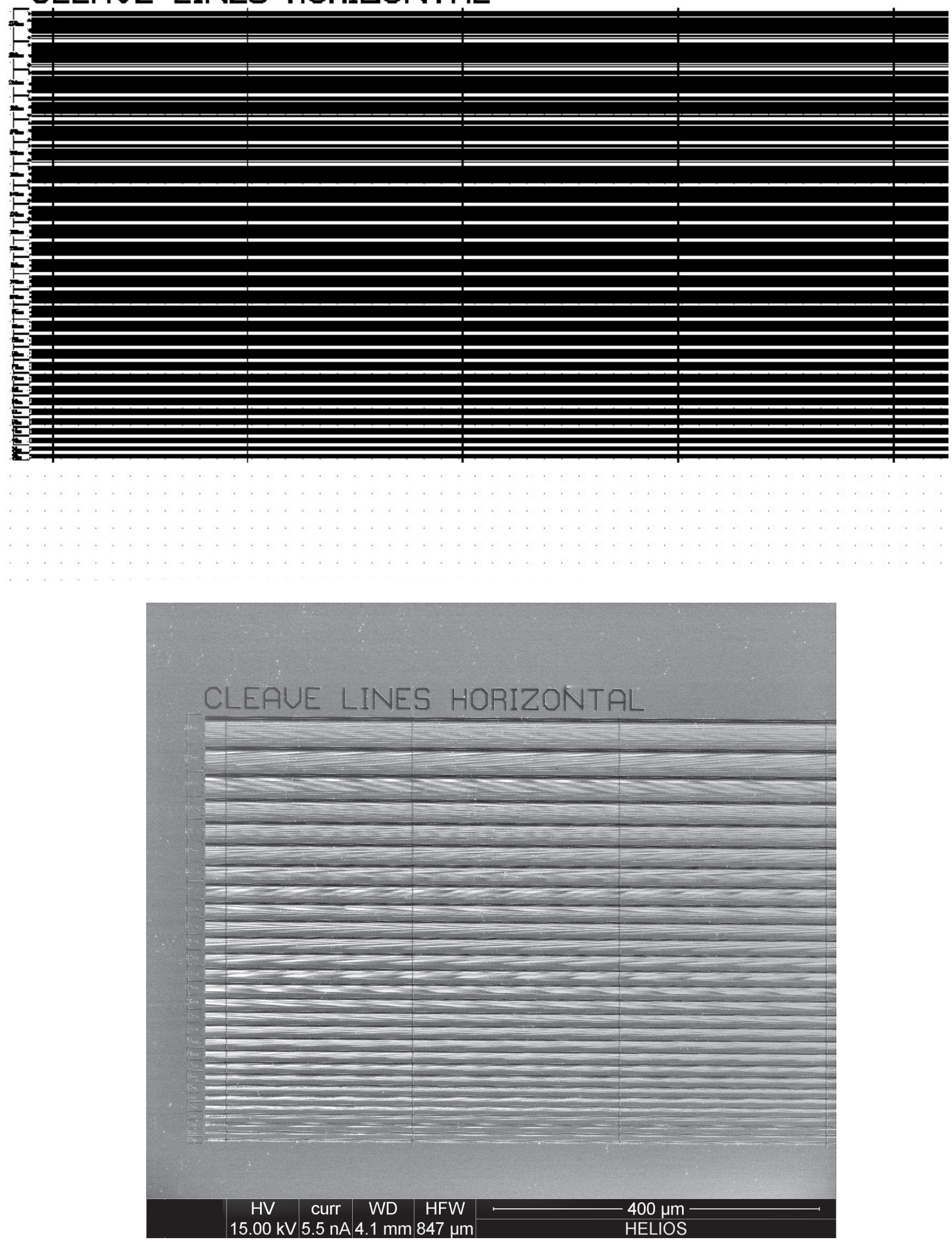

Plate 118 - Pattern 7-2 - Cleave Lines. 
Plate 119 - Pattern 7-2 - Cleave Lines. (top) Higher FOV GDS II line drawing of several of the horizontal cleave line targets found on Pattern 7-2. (bottom) SEM micrograph of a similar area. 

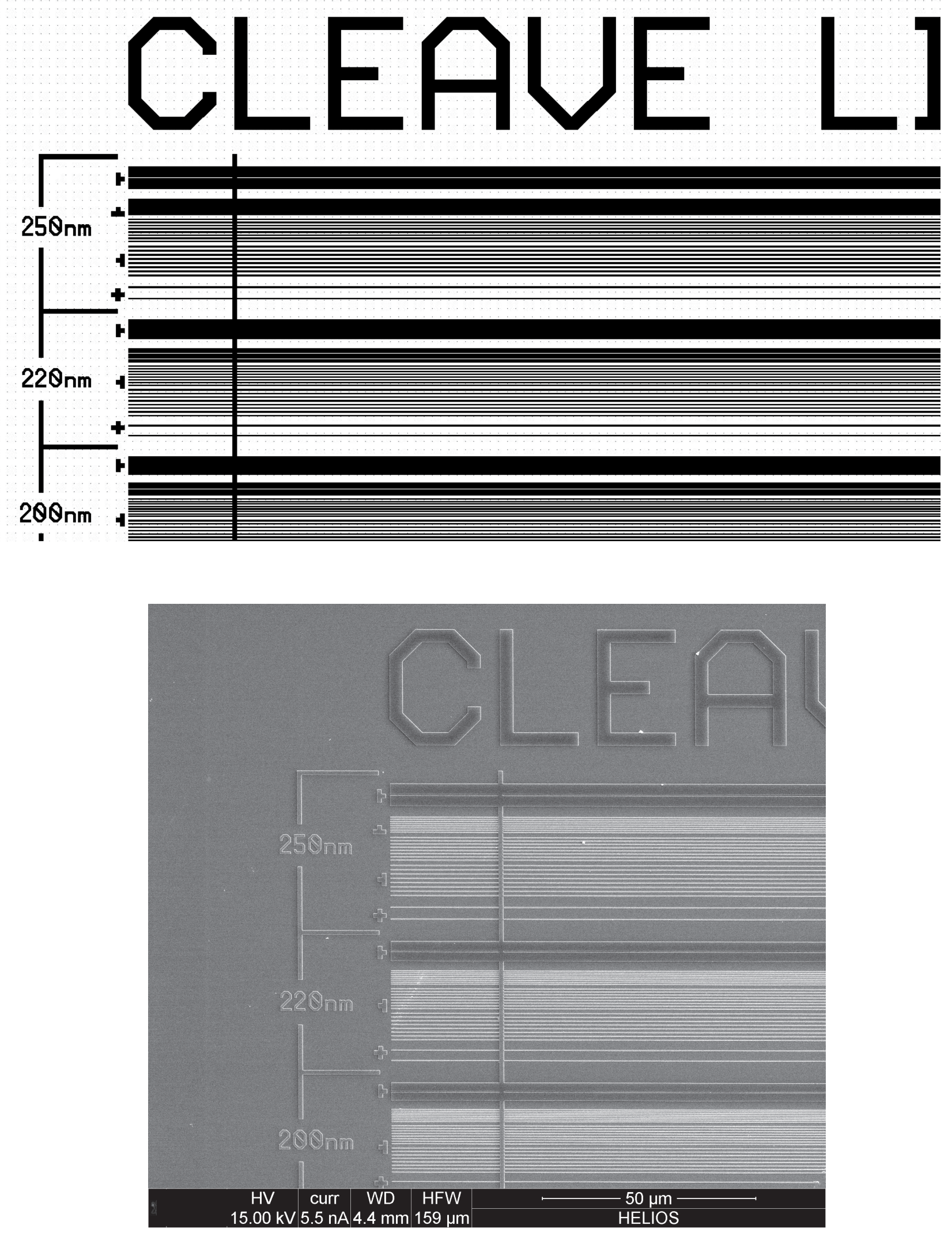

Plate 119 - Pattern 7-2 - Cleave Lines. 
Plate 120 - Pattern 7-3 - Multisize Scatterometry. (top) Wide FOV GDS II line drawing of several of the horizontal multisize scatterometry line targets found on Pattern 7-3. (bottom) SEM micrograph of a similar area. 

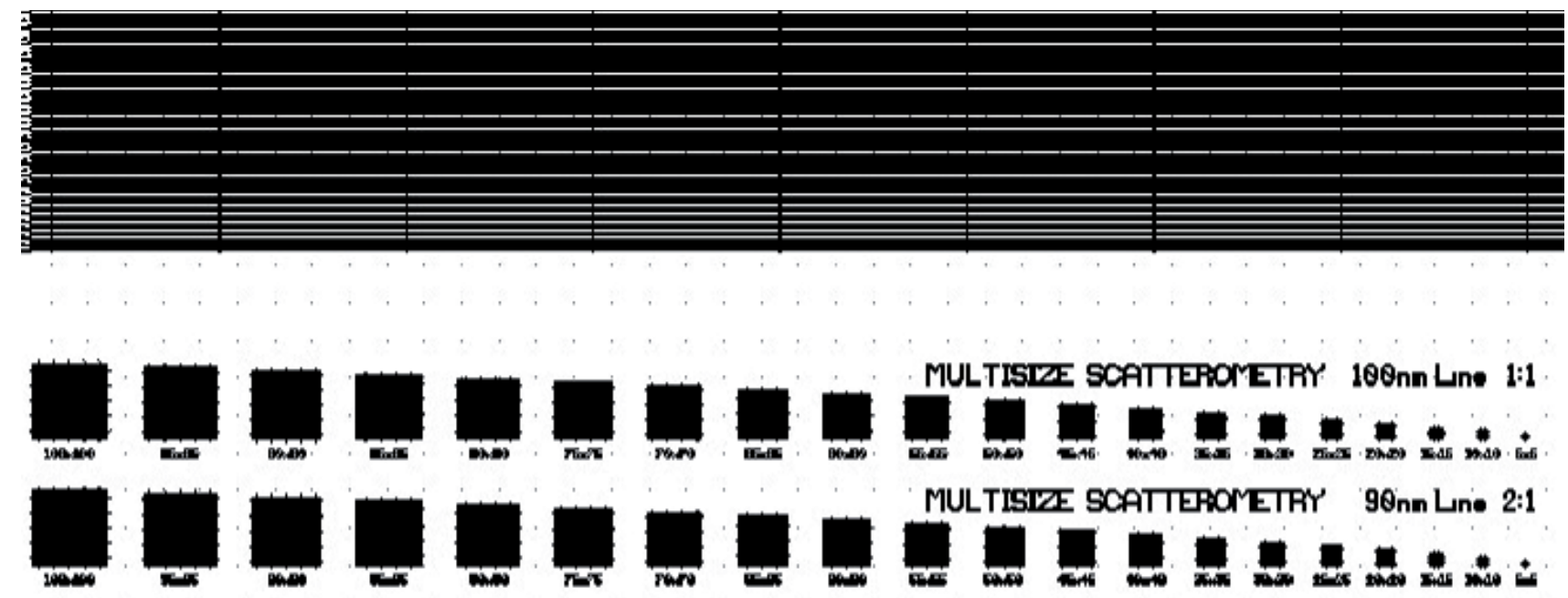

SCATTEROMETRY DEFECT 9QNM 2:1
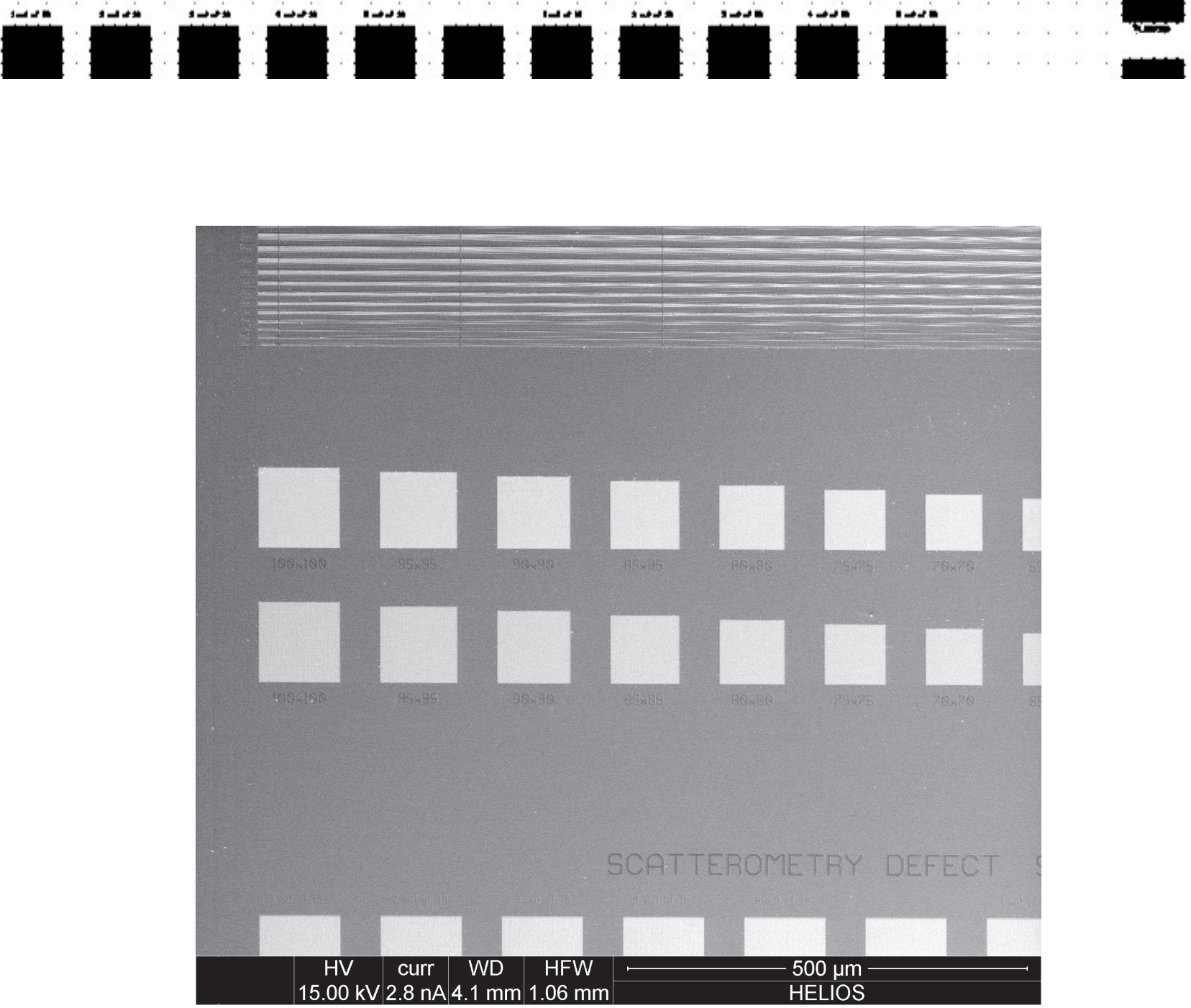

Plate 120 - Pattern 7-3 - Multisize Scatterometry 
Plate 121 - Pattern 7-3 - Scatterometry Defect. (top) Wide FOV GDS II line drawing of Pattern 7-3. (bottom) SEM micrograph of a similar area. 


\section{?E SCATTEROMETI
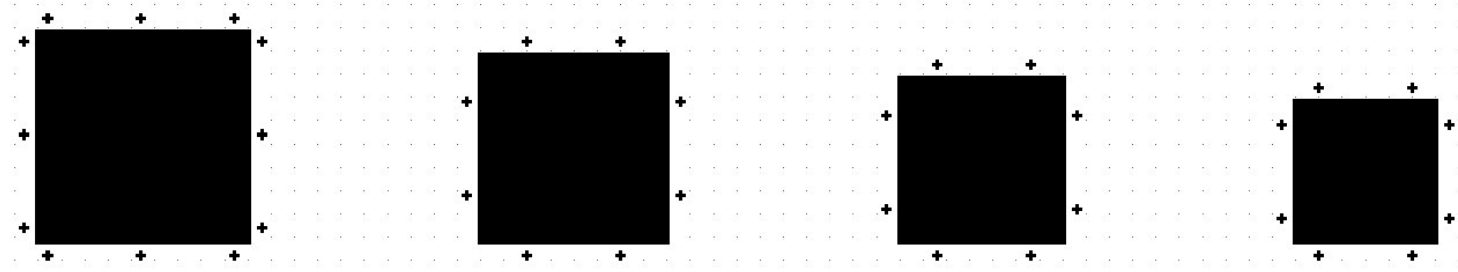 \\ $45 \times 45$ \\ $40 \times 40$ \\ $35 \times 35$ \\ $30 \times 30$}

\section{ZE SCATTEROMETI}

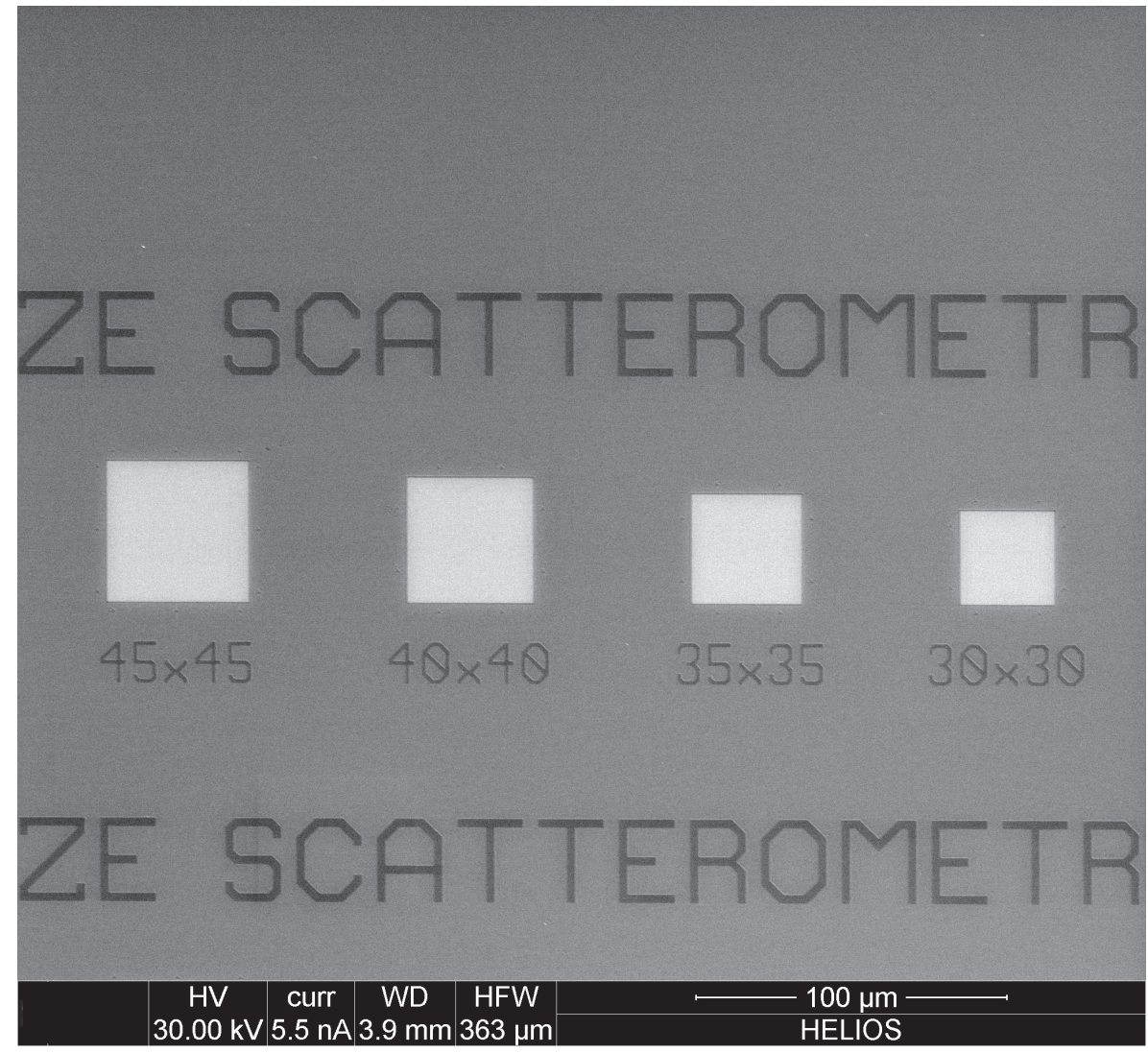

Plate 121 - Pattern 7-3 - Scatterometry Defect 
Plate 122 - Pattern 7-3 - Scatterometry Defect. (top) Low HFW SEM micrograph.of pattern 7-3. (bottom) Higher HFW SEM micrograph of a similar area. 

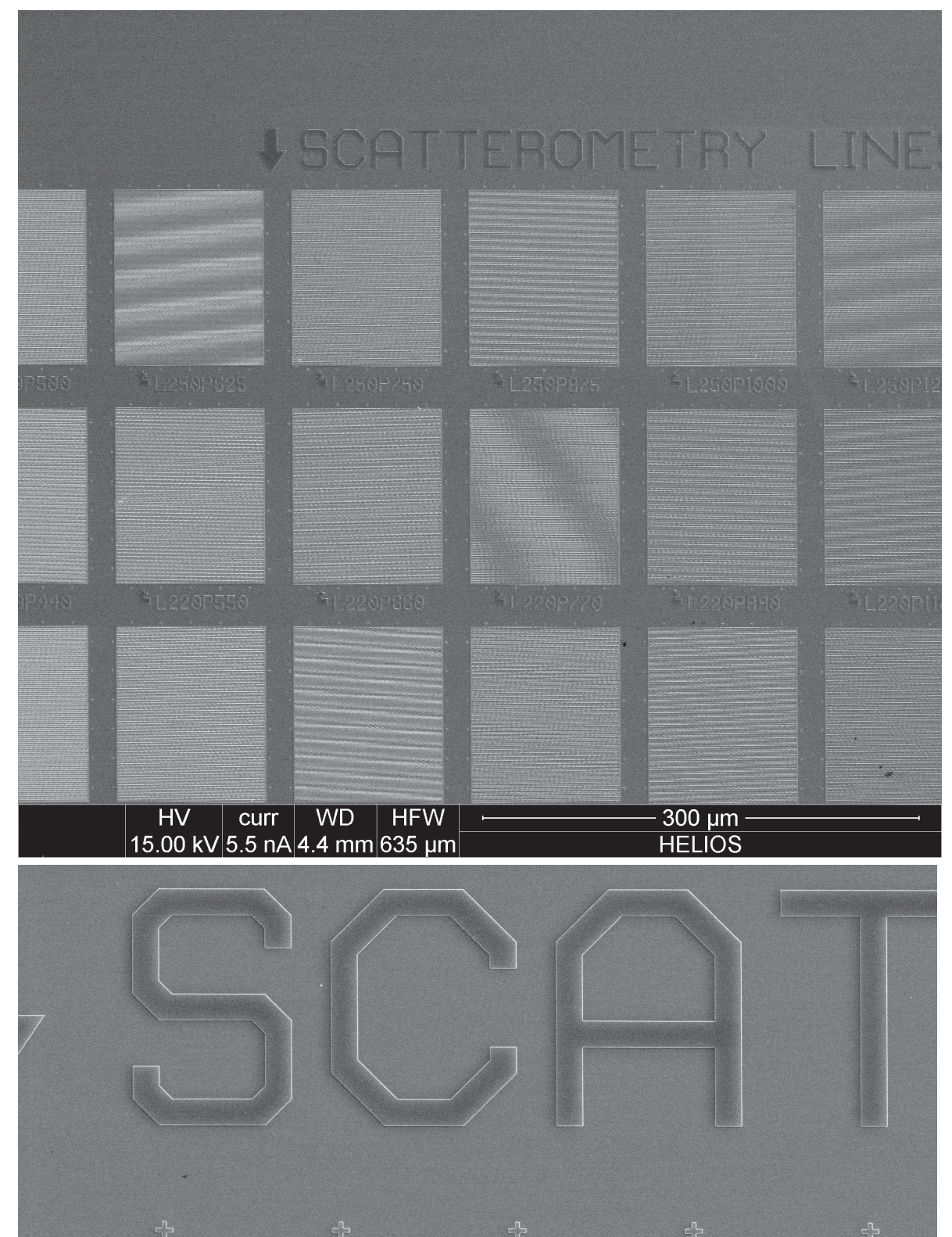

3

3

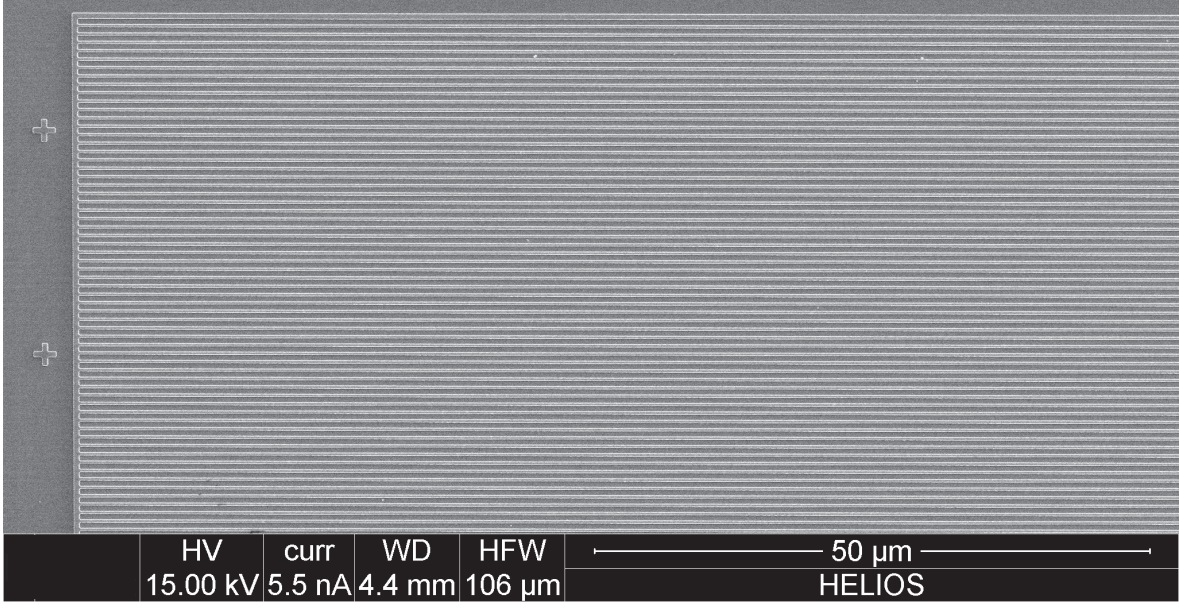

Plate 122 - Pattern 7-3 - Scatterometry Defect. 
Plate 123 - Pattern 7-4 - Scatterometry Defect. (top) Wide FOV GDS II line drawing of Pattern 7-4. (bottom) SEM micrograph of a similar area. 


\section{SCATTEROMETRY DEFECT 9ONM 2:1}

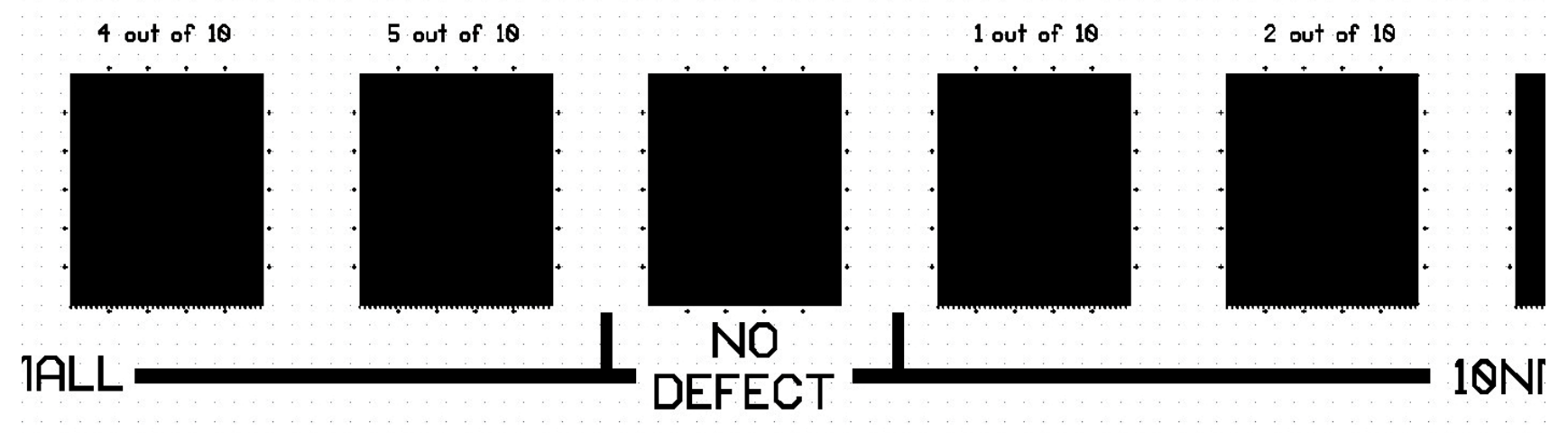

\section{SCATTEROMETRY DEFECT 1OONM 1:1}

4 out of 10

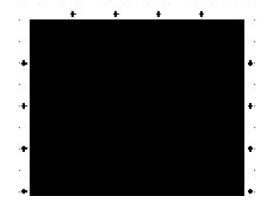

5 out of 10

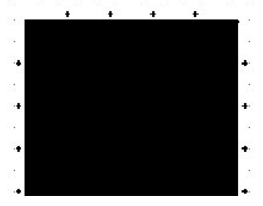

1 out of 10
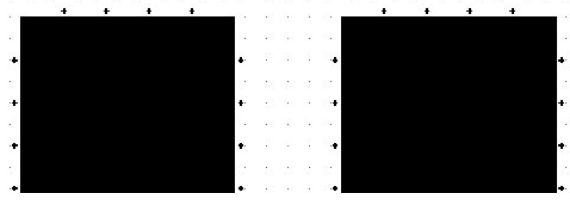

2 out of 18
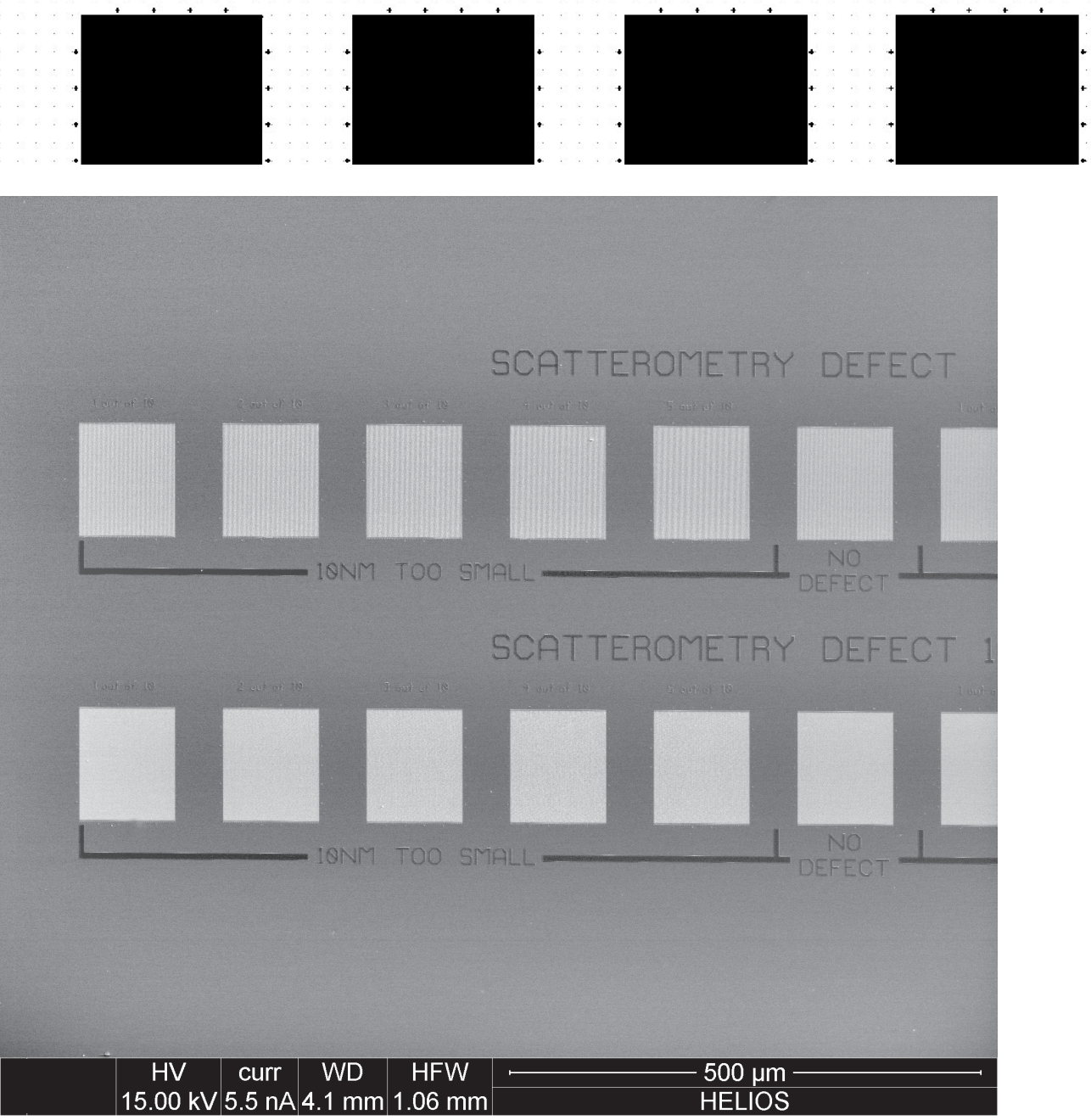

Plate 123 - Pattern 7-4 - Scatterometry Defect. 
Plate 124 - Pattern 7-4 - Scatterometry Defect. (top) Low HFW SEM micrograph of Pattern 7-4. (bottom) Higher HFW SEM micrograph of the finer detail of the patterns. 

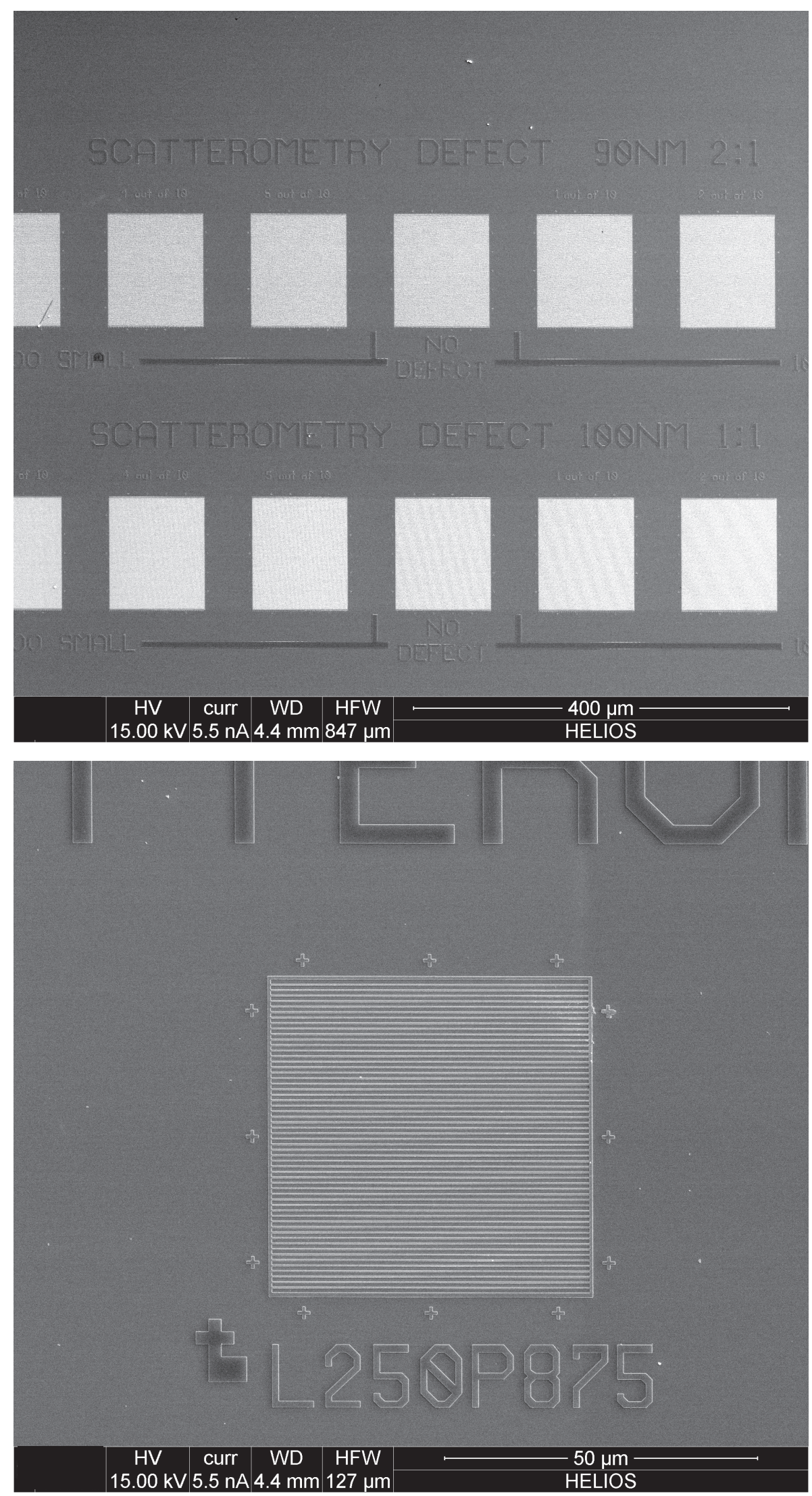

$15.00 \mathrm{kV} 5.5 \mathrm{nA} 4.4 \mathrm{~mm} 127 \mu \mathrm{m} \quad$ HELIOS

Plate 124 - Pattern 7-4 - Scatterometry Defect. 
Plate 125 - Pattern 7-5 - Phase Imbalance. (top) Wide FOV GDS II line drawing of the phase imbalance pattern. (bottom) SEM micrograph of a similar area. 


\section{PHASE IMBALANCE}

16ONM 11

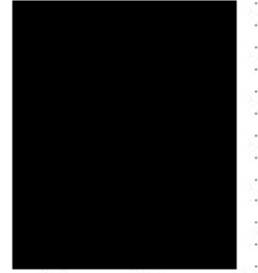

Phase Area $20 \mathrm{~nm}$ Largor
$100 \mathrm{NM} 11$

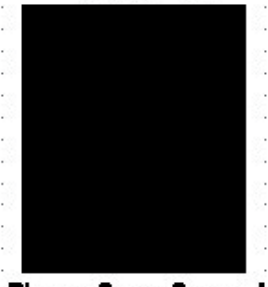

Phaee Areo Correot
100 NM 11

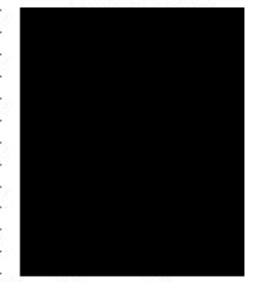

Phaes Area 20nm Smallor
SNM 21

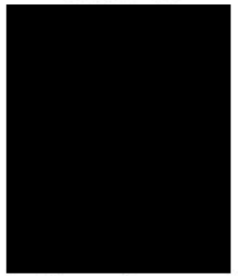

Phaes Area 20nm Larger
9ONM 21

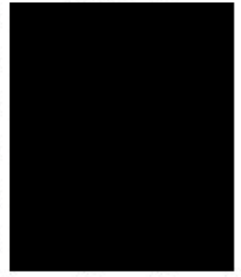

Phaes Area Corroot

PHASE IMBALANCE
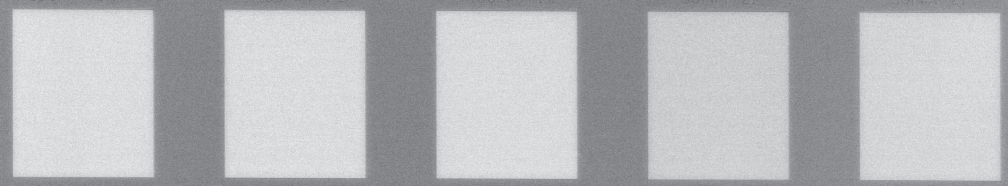

\MULTIPITCH SCATTER LINES \

HV curr WD HFW

$30.00 \mathrm{kV} 5.5 \mathrm{nA} 3.9 \mathrm{~mm} 847 \mu \mathrm{m}$

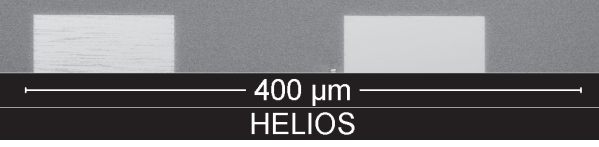

Plate 125 - Pattern 7-5 - Phase Imbalance 
Plate 126 - Pattern 7-5 - Phase Imbalance. (top) Wide FOV GDS II line drawing of the phase imbalance pattern. (bottom) SEM micrographs of a similar area. 


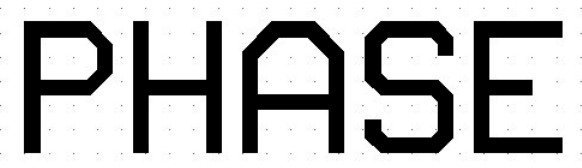

10ONM 11

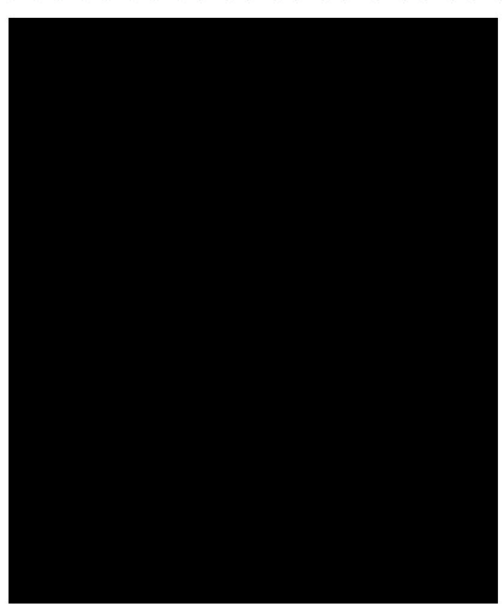

Phase Area $28 \mathrm{~nm}$ Larger

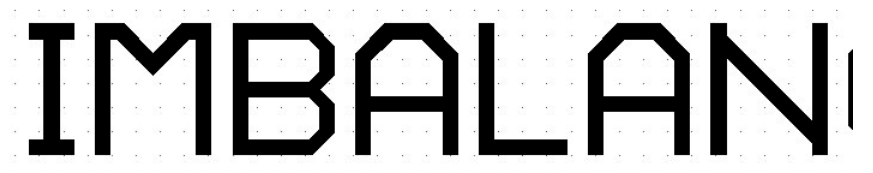
10ONM 11

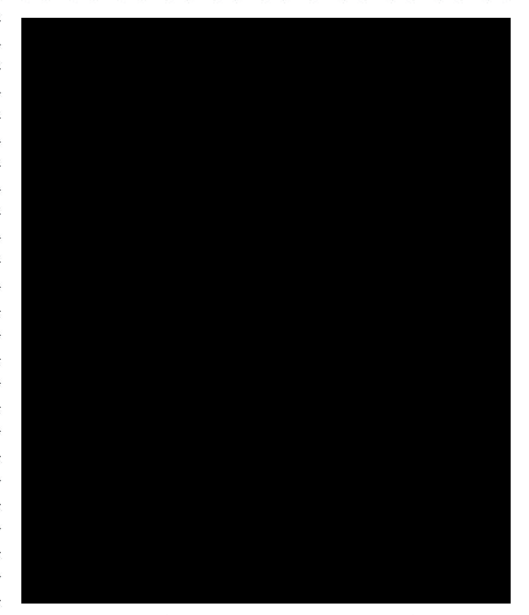

Phase Area Correct

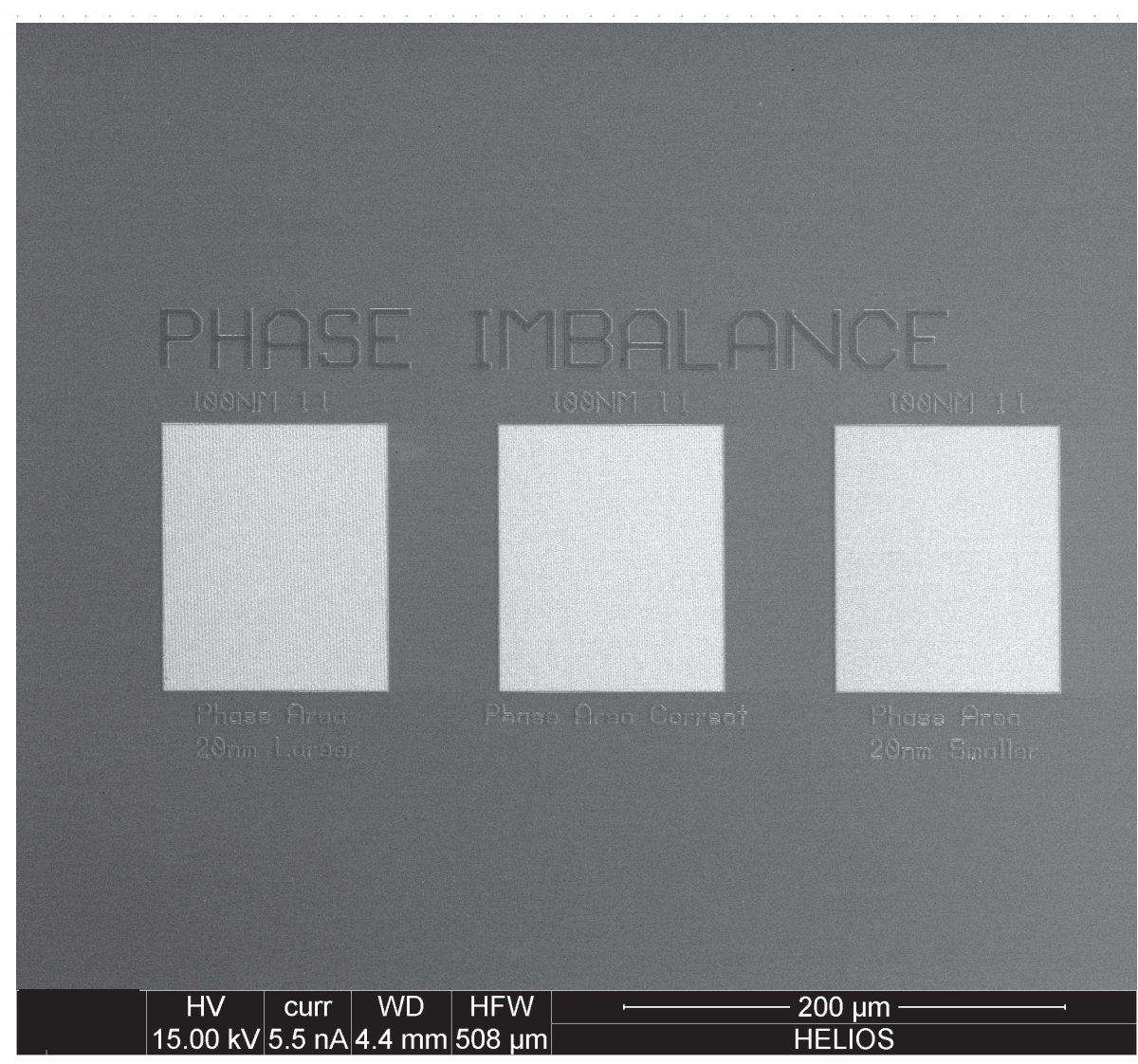

Plate 126 - Pattern 7-5 - Phase Imbalance. 
Plate 127 - Pattern 7-5 - Phase Imbalance. (top) Higher HFW SEM micrographs of Pattern 7-5. (bottom) Higher HFW of one of the phase imbalance patterns. 

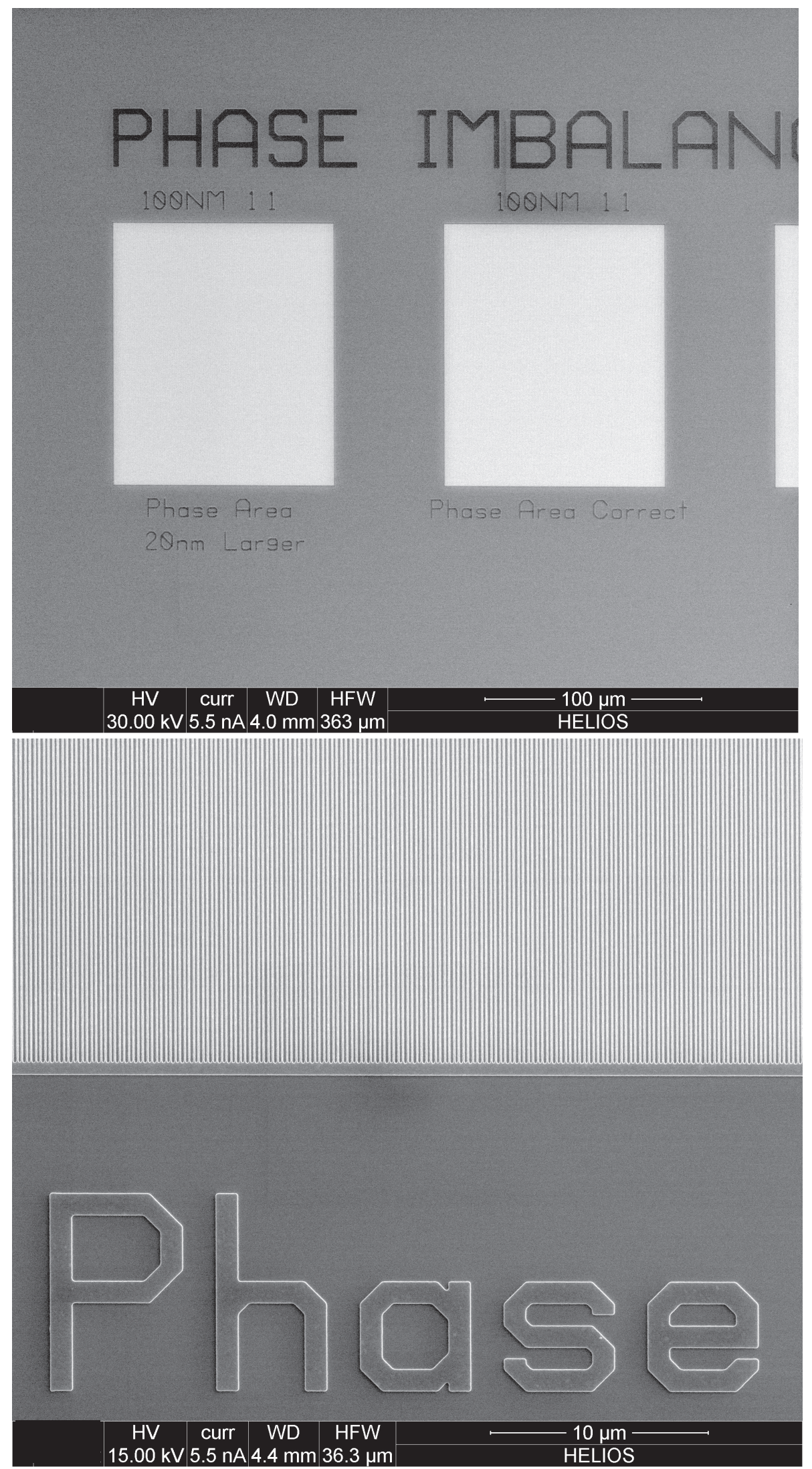

Plate 127 - Pattern 7-5 - Phase Imbalance 
Plate 128 - Pattern 7-6 - Multiple Scatter Lines. (top) Wide FOV GDS II line drawing of several of the multiple scatter lines targets found on Pattern 7-6. (bottom) SEM micrograph of a similar area. 
MUUTIPITCH SCATTER LINES †
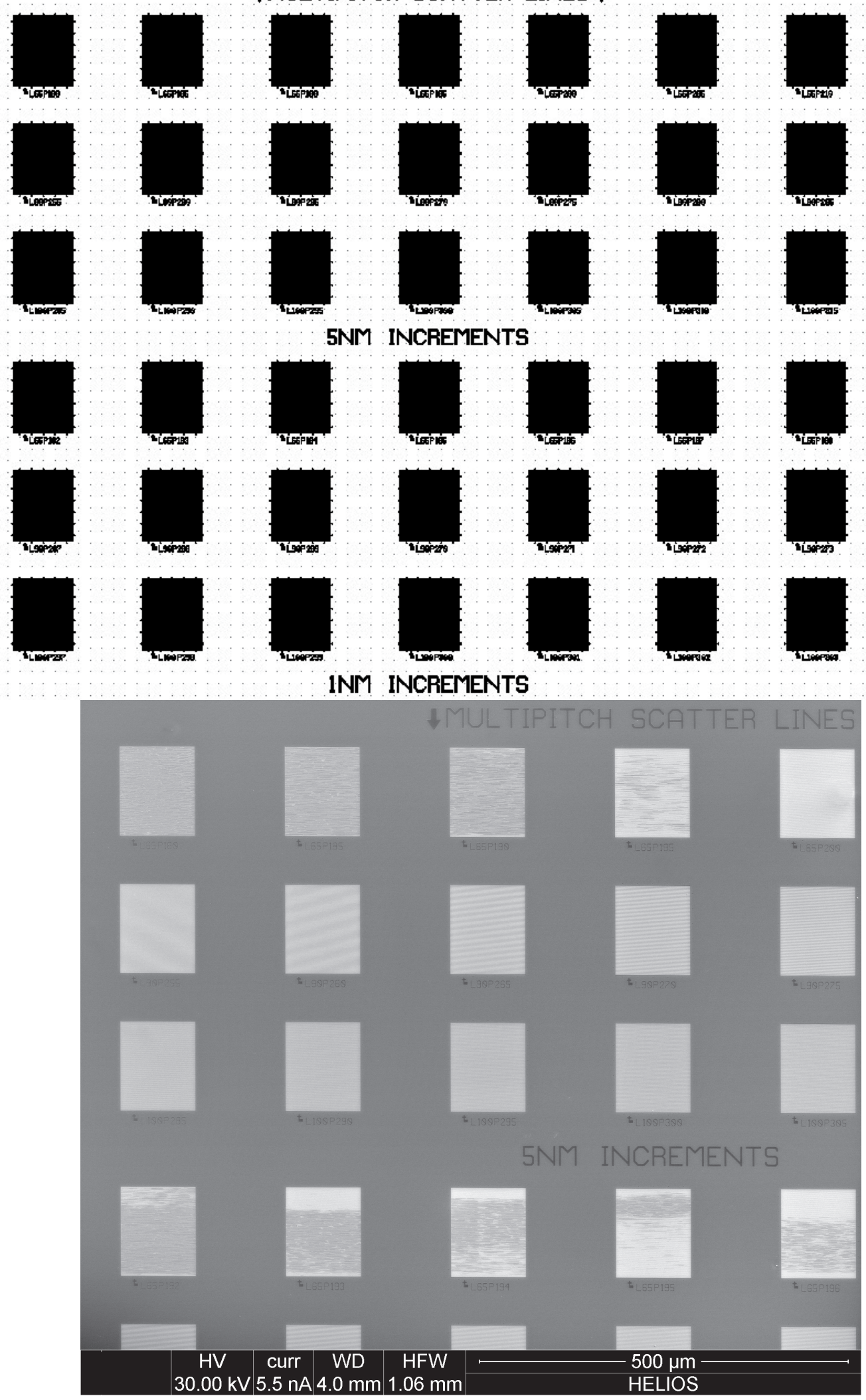

Plate 128 - Pattern 7-6 - Multiple Scatter Lines. 
Plate 129 - Pattern 7-6 - Multiple Scatter Lines. (top) Wide FOV GDS II line drawing of L100P295 pattern found on Pattern 7-6. (bottom) SEM micrograph of a similar area. 

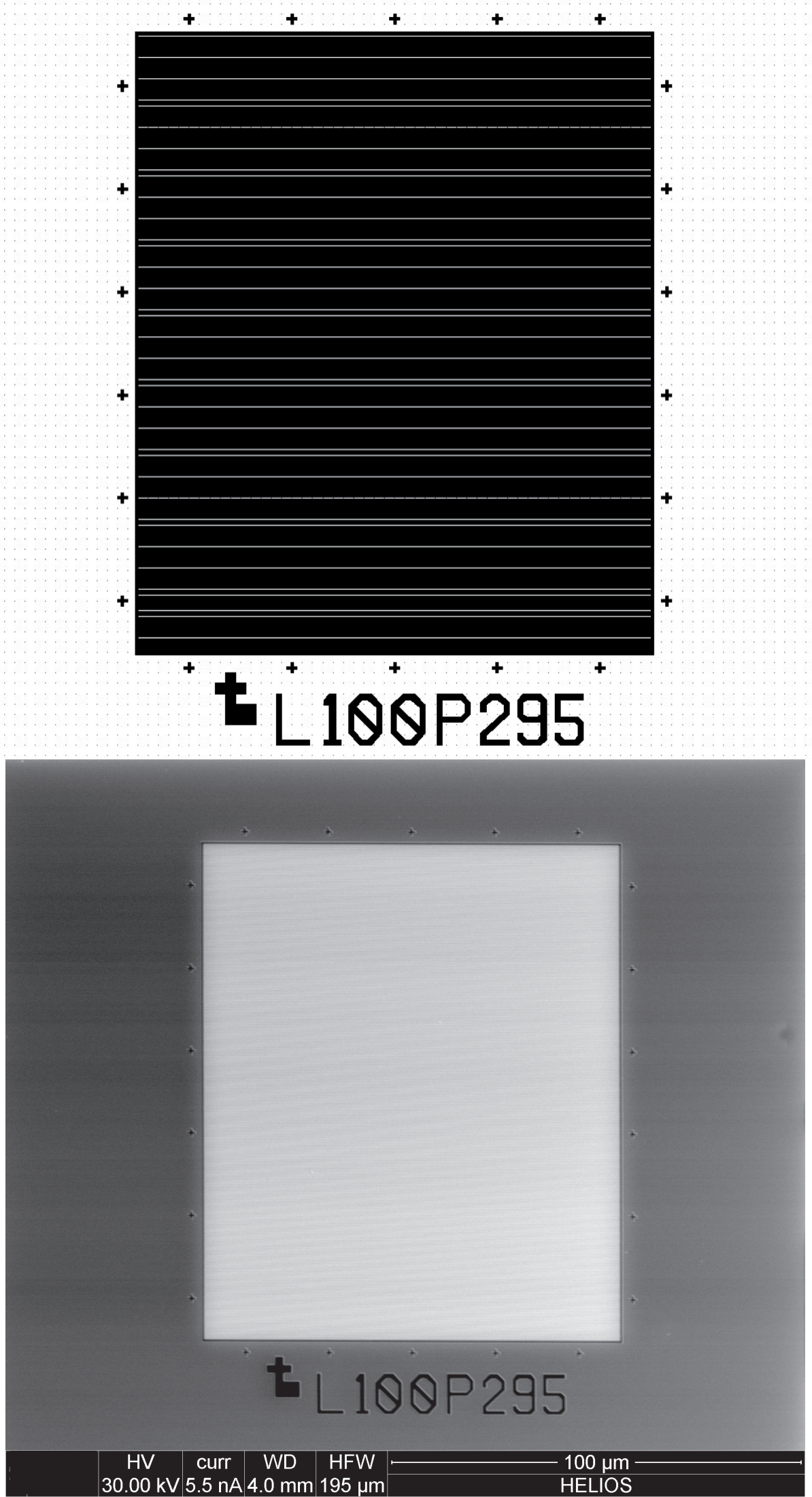

Plate 129 - Pattern 7-6 - Multiple Scatter Lines. 
Plate 130 - Pattern 7-6 - Multiple Scatter Lines. SEM micrograph at a smaller HFW of the lines found on Pattern 7-6. 


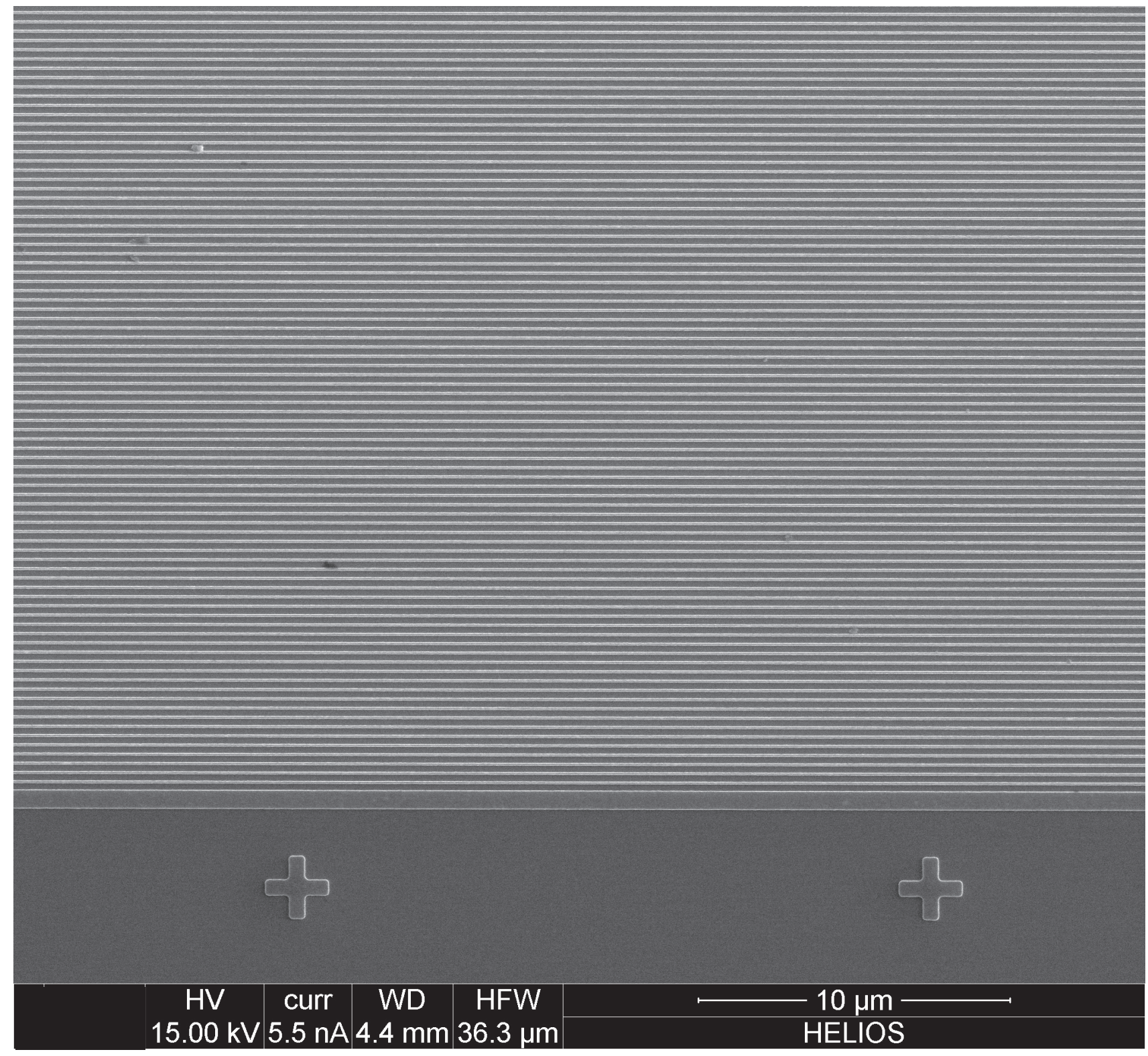

Plate 130 - Pattern 7-6 - Multiple Scatter Lines. 